Copyright: The author, Ali Alqahtani, 2021.

\title{
Deep Clustering and Deep Network Compression
}

\author{
Ali Alqahtani \\ Submitted to Swansea University in fulfilment \\ of the requirements for the Degree of Doctor of Philosophy

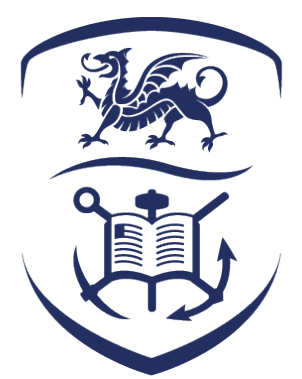

\section{Swansea University Prifysgol Abertawe}

Department of Computer Science

Swansea University

July 1,2021 


\section{Declaration}

This work has not been previously accepted in substance for any degree and is not being concurrently submitted in candidature for any degree.

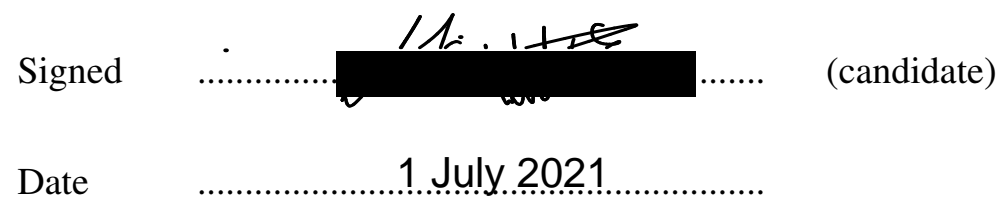

\section{Statement 1}

This thesis is the result of my own investigations, except where otherwise stated. Other sources are acknowledged by footnotes giving explicit references. A bibliography is appended.

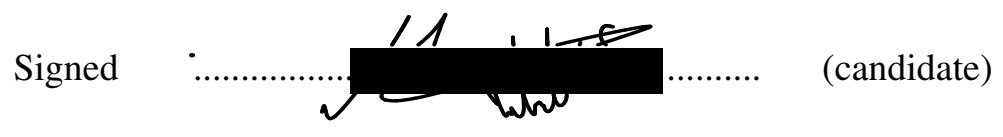

Date 1. July. 2021

\section{Statement 2}

I hereby give my consent for my thesis, if accepted, to be available for photocopying and for inter-library loan, and for the title and summary to be made available to outside organisations.

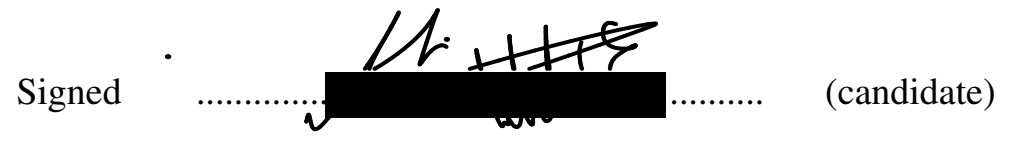

Date 1 July 2021 
To my parents and my wife.

I would also like to dedicate this thesis to my grandmother and grandfather, who passed away during the final stage of my Ph.D. study. 


\section{Abstract}

The use of deep learning has grown increasingly in recent years, thereby becoming a muchdiscussed topic across a diverse range of fields, especially in computer vision, text mining, and speech recognition. Deep learning methods have proven to be robust in representation learning and attained extraordinary achievement. Their success is primarily due to the ability of deep learning to discover and automatically learn feature representations by mapping input data into abstract and composite representations in a latent space. Deep learning's ability to deal with high-level representations from data has inspired us to make use of learned representations, aiming to enhance unsupervised clustering and evaluate the characteristic strength of internal representations to compress and accelerate deep neural networks.

Traditional clustering algorithms attain a limited performance as the dimensionality increases. Therefore, the ability to extract high-level representations provides beneficial components that can support such clustering algorithms. In this work, we first present DeepCluster, a clustering approach embedded in a deep convolutional auto-encoder. We introduce two clustering methods, namely DCAE-Kmeans and DCAE-GMM. The DeepCluster allows for data points to be grouped into their identical cluster, in the latent space, in a joint-cost function by simultaneously optimizing the clustering objective and the DCAE objective, producing stable representations, which is appropriate for the clustering process. Both qualitative and quantitative evaluations of proposed methods are reported, showing the efficiency of deep clustering on several public datasets in comparison to the previous state-of-the-art methods.

Following this, we propose a new version of the DeepCluster model to include varying degrees of discriminative power. This introduces a mechanism which enables the imposition of regularization techniques and the involvement of a supervision component. The key idea of our approach is to distinguish the discriminatory power of numerous structures when searching for a compact structure to form robust clusters. The effectiveness of injecting various levels of discriminatory powers into the learning process is investigated alongside the exploration and analytical study of the discriminatory power obtained through the use of two discriminative 
attributes: data-driven discriminative attributes with the support of regularization techniques, and supervision discriminative attributes with the support of the supervision component. An evaluation is provided on four different datasets.

The use of neural networks in various applications is accompanied by a dramatic increase in computational costs and memory requirements. Making use of the characteristic strength of learned representations, we propose an iterative pruning method that simultaneously identifies the critical neurons and prunes the model during training without involving any pre-training or fine-tuning procedures. We introduce a majority voting technique to compare the activation values among neurons and assign a voting score to evaluate their importance quantitatively. This mechanism effectively reduces model complexity by eliminating the less influential neurons and aims to determine a subset of the whole model that can represent the reference model with much fewer parameters within the training process. Empirically, we demonstrate that our pruning method is robust across various scenarios, including fully-connected networks (FCNs), sparsely-connected networks (SCNs), and Convolutional neural networks (CNNs), using two public datasets.

Moreover, we also propose a novel framework to measure the importance of individual hidden units by computing a measure of relevance to identify the most critical filters and prune them to compress and accelerate CNNs. Unlike existing methods, we introduce the use of the activation of feature maps to detect valuable information and the essential semantic parts, with the aim of evaluating the importance of feature maps, inspired by novel neural network interpretability. A majority voting technique based on the degree of alignment between a semantic concept and individual hidden unit representations is utilized to evaluate feature maps' importance quantitatively. We also propose a simple yet effective method to estimate new convolution kernels based on the remaining crucial channels to accomplish effective CNN compression. Experimental results show the effectiveness of our filter selection criteria, which outperforms the state-of-the-art baselines.

To conclude, we present a comprehensive, detailed review of time-series data analysis, with emphasis on deep time-series clustering (DTSC), and a founding contribution to the area of applying deep clustering to time-series data by presenting the first case study in the context of movement behavior clustering utilizing the DeepCluster method. The results are promising, showing that the latent space encodes sufficient patterns to facilitate accurate clustering of movement behaviors. Finally, we identify state-of-the-art and present an outlook on this important field of DTSC from five important perspectives. 


\section{Acknowledgements}

First of all, I would like to extend my sincerest appreciation and gratitude to my supervisor, Prof. Xianghua Xie, for his supervision, guidance, support, and inspiration. Over the years, he has been a constant source of advice and encouragement, for which I will always be thankful. Without his tireless encouragement and help, this thesis would not have been possible. I would like to thank him for the support and discussions that have assisted me in developing my professional skills. He has all my thanks for pushing me to do my best. I am also grateful to my second supervisor, Prof. Mark W. Jones for his help and support.

I would also like to thank all of my colleagues in the SwanseaVision Lab. Thanks especially to Dr. Essa Ehab, Dr. Jingjing Deng, Dr. Michael Edwards, Majedaldein Almahasneh, Hanchi Ren, Hassan Eshkiki, and Michael Kenning for their helpful discussions and support throughout my Ph.D.

I am also thankful to all of my friends, Mohammed Ali, Abdulrahim Meshari, Majed Bin Othayman, and Khalid Alharthi for their continuous support.

Finally, I would also like to express my gratitude to my family, especially my amazing parents, my wonderful wife (for her patience and support in helping me pursue something that I love, despite the difficulties), my daughters (Taraf, Arob, and Hoor), my sisters, and my brothers for their encouragement and support throughout the years; they always believed in me and pushed me to succeed. 


\section{Contents}

$\begin{array}{lc}\text { List of Publications } & \text { ix }\end{array}$

$\begin{array}{ll}\text { List of Tables } & \mathbf{x}\end{array}$

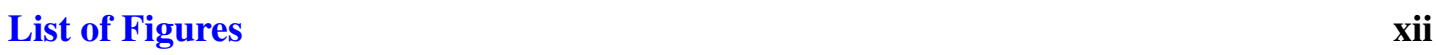

1 Introduction 1

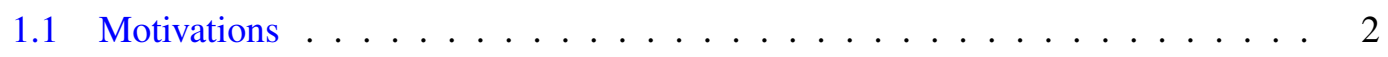

1.1.1 Deep Clustering . . . . . . . . . . . . . . . . 3

1.1.2 Learning Discriminatory Deep Clustering Models . . . . . . . . . . . . 4

1.1.3 Deep Neural Network Compression and Acceleration . . . . . . . . . . 4

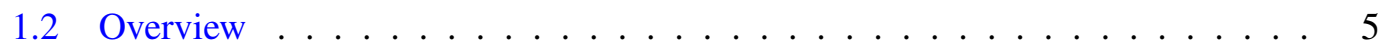

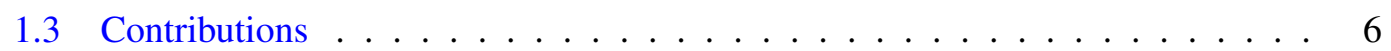

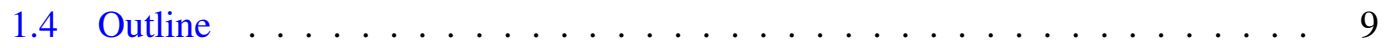

2 Background $\quad 11$

2.1 Introduction . . . . . . . . . . . . . . . . . . . . . . . . . 12

2.2 Deep Learning . . . . . . . . . . . . . . . . . . . . . . . 13

2.2.1 Neural Networks . . . . . . . . . . . . . . . . . . . . . . 13

2.2.2 Convolutional Neural Networks _. . . . . . . . . . . . . . . . 21

2.2.3 Modern Convolutional Neural Networks . . . . . . . . . . . . . . . . . 24

2.2 .4 Auto-encoders . . . . . . . . . . . . . . . . . . . . . 28

2.2.5 Advances in Deep Learning . . . . . . . . . . . . . . . . . . . 30

2.2.6 Visualizing, Explaining and Interpreting Deep Learning _. . . . . . 31

2.3 Clustering . . . . . . . . . . . . . . . . . . . . . . . . . 34

2.3.1 Conventional Clustering Methods . . . . . . . . . . . . . . . . . 34 
2.3.2 Deep Clustering Methods . . . . . . . . . . . . . . . . . . 38

2.3.3 Clustering Supervision . . . . . . . . . . . . . . . . . . . . . 44

2.4 Deep Neural Network Compression and Acceleration . . . . . . . . . . . . . . 45

2.4.1 Pruning Methods . . . . . . . . . . . . . . . . . . . . . . . 47

2.4.2 Quantization Methods . . . . . . . . . . . . . . . . . 51

2.4.3 Low-rank Factorization Methods . . . . . . . . . . . . . . . . . . . . 52

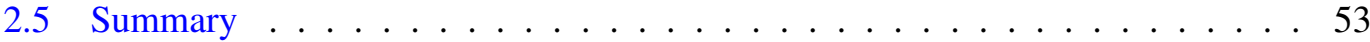

3 DeepCluster: A Deep Convolutional Auto-encoder with Embedded Clustering $\quad \mathbf{5 5}$

3.1 Introduction . . . . . . . . . . . . . . . . . . . . 56

3.2 Proposed Methods ． . . . . . . . . . . . . . . . . . . . . . 57

3.2.1 Deep Convolutional Auto-encoder (DCAE) . . . . . . . . . . . . . 58

3.2.2 DeepCluster: A Deep Convolutional Auto-encoder with Embedded Clustering . . . . . . . . . . . . . . . . . . 59

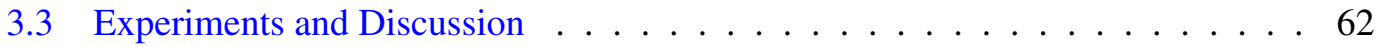

3.3 .1 Datasets . . . . . . . . . . . . . . . . . . . . . 62

3.3.2 Network Architectures . . . . . . . . . . . . . . . . . . . . 63

3.3.3 Evaluation Metrics . . . . . . . . . . . . . . . . . . . 65

3.3.4 Implementation Details . . . . . . . . . . . . . . . . 65

3.3.5 Baseline Methods . . . . . . . . . . . . . . . . . . . . . . . . . 66

3.3.6 Quantitative Results and Analysis . . . . . . . . . . . . . . . . . 66

3.3.7 Visualization Results and Analysis . . . . . . . . . . . . . . . . . 68

3.4 Summary . . . . . . . . . . . . . . . . . . . . . 73

4 Learning Discriminatory Deep Clustering Models $\quad 75$

4.1 Introduction . . . . . . . . . . . . . . . . . . . . 76

4.2 Proposed Methods . . . . . . . . . . . . . . . . . . . 78

4.2.1 DeepCluster: A DCAE with Embedded Clustering . . . . . . . . . . . 79

4.2 .2 Architecture . . . . . . . . . . . . . . . . . . . . . . . . 79

4.2.3 Graph-Based Activity Regularization (GBAR) $\ldots \ldots \ldots$. . . . . . 80

4.2.4 Data Augmentation (DA) . . . . . . . . . . . . . . . . 81

4.2.5 Extended Output Layer and Different Levels of Supervision . . . . . . 82

4.3 Experimental Results . . . . . . . . . . . . . . . . . . . . . . . 83

4.3.1 Regularizations for DeepCluster . . . . . . . . . . . . . . . . . . 84 
4.3.2 Learning Discriminatory Deep Clustering Models Through Different Levels of supervision . . . . . . . . . . . . . . . . . 87

4.3.3 Deep Clustering Through Various Levels of Supervision . . . . . . . . 91

4.4 Summary . . . . . . . . . . . . . . . . . . . . . . . . . 93

5 Reducing Neural Network Parameters via Neuron-based Iterative Pruning 97

5.1 Introduction . . . . . . . . . . . . . . . . . . . . . . 98

5.2 Proposed Method . . . . . . . . . . . . . . . . . . . . . . . . 99

5.2.1 Importance of Individual Neurons via Majority voting (MV) . . . . . 100

5.2 .2 Pruning algorithm . . . . . . . . . . . . . . . . . 101

5.3 Experiments and Discussion $\ldots \ldots \ldots$. . . . . . . . . . . 102

5.3.1 Measuring Neuron Importance via Ablation . . . . . . . . . . . . . . 104

5.3.2 Pruning Redundant Neurons During Training . . . . . . . . . . . . 106

5.3.3 Integrating our Pruning Method to Sparsely-Connected Networks (SCNs) 108

5.3.4 Extension to Convolutional Neural Networks (CNNs) . . . . . . . . . . 109

5.4 Summary . . . . . . . . . . . . . . . . . . . . . . . 110

6 Pruning CNN Filters via Quantifying the Importance of Deep Visual Representations 113

6.1 Introduction . . . . . . . . . . . . . . . . . . . . . . . . . . . 114

6.2 Proposed Method . . . . . . . . . . . . . . . . . . . . . 117

6.2.1 Overview of our Pruning Methodology . . . . . . . . . . . . 117

6.2 .2 Scoring Channel Importance Method . . . . . . . . . . . . . . 118

6.2 .3 Majority voting (MV) Method . . . . . . . . . . . . . . . . 120

6.2.4 Kernels Estimation Method . . . . . . . . . . . . . . . . . . . . 122

6.3 Experiments and Discussion . . . . . . . . . . . . . . 123

6.3 .1 VGG16 on CIFAR-10 . . . . . . . . . . . . . . . . . . . 129

6.3.2 ResNet-20/ResNet-32 on CIFAR-10 . . . . . . . . . . . . . . . . 131

6.3.3 ResNet-20/ResNet-32 on CUB-200 . . . . . . . . . . . . . . . . 132

6.3 .4 ResNet-50 on ImageNet . . . . . . . . . . . . . . . . . . 134

6.3.5 Feature Map Visualization . . . . . . . . . . . . . . . . . . . 136

6.3.6 Comparison with Filter Selection Criteria . . . . . . . . . . . . . 137

6.3.7 Comparison of Kernal Estimation (KE) vs. Fine-Tuning (FT) . . . . . . 142

6.4 Summary . . . . . . . . . . . . . . . . . . . . . . 143 
7 Deep Time-Series Clustering $\quad 145$

7.1 Introduction . . . . . . . . . . . . . . . . . . . . . . . . . . . 146

7.2 Time-series Data Type . . . . . . . . . . . . . . . . . . . . . . 147

7.2 .1 Univariate . . . . . . . . . . . . . . . . . . . . . . . . . . . . 147

7.2.2 Multivariate . . . . . . . . . . . . . . . . . . . . . 147

7.2 .3 Tensor Fields . . . . . . . . . . . . . . . . . . . . . . . . . . . 148

7.2 .4 Multifield . . . . . . . . . . . . . . . . . . . . . . . . . . . 149

7.3 Conventional Time-series Analysis . . . . . . . . . . . . . . . . . . . . . . . . 149

7.3.1 Similarity Measures and Features Extraction _ . . . . . . . . . 151

7.3.2 Conventional Clustering Algorithms . . . . . . . . . . . . . . . 155

7.4 DeepCluster Method Applied to Biological Time-series Data: A Case Study . . 157

7.4.1 Network Architectures for ICBD . . . . . . . . . . . . . . . . . . . . . 158

7.4.2 Imperial Cormorant Birds Dataset (ICBD) and Pre-processing . . . . . 161

7.4 .3 Experiment and Discussion . . . . . . . . . . . . . . . . . . 163

7.5 State-of-the-art and Outlook . . . . . . . . . . . . . . . . . . . . 165

7.5.1 Different Network Architectures . . . . . . . . . . . . . . . . 165

7.5.2 Different Clustering Methods . . . . . . . . . . . . . . . . . 168

7.5.3 Deep Learning Heuristics ～. . . . . . . . . . . . . . . . . . . . . 169

7.5.4 DTSC Applictions . . . . . . . . . . . . . . . . . . . . . 170

7.5 .5 DTSC Benchmarks . . . . . . . . . . . . . . . . . . . . 171

7.6 Summary . . . . . . . . . . . . . . . . . . . . . . . 171

8 Conclusions and Future Work 173

8.1 Conclusions . . . . . . . . . . . . . . . . . . . . . . . 174

8.2 Future Work . . . . . . . . . . . . . . . . . . . . . . . . 176

$\begin{array}{ll}\text { Bibliography } & 181\end{array}$ 


\section{List of Publications}

The following is a list of published papers as a result of the work in this thesis. An outline of contributions related to the main body of the thesis work can be found in Section 1.3.

1. A. Alqahtani, X. Xie, Mark W. Jones, and E. Essa. Pruning CNN Filters via Quantifying the Importance of Deep Visual Representations. Computer Vision and Image Understanding (CVIU), 2021. [1]

2. A. Alqahtani, X. Xie, E. Essa, and Mark W. Jones. Neuron-based Network Pruning Based on Majority Voting. International Conference on Pattern Recognition (ICPR), 2020. [2]

3. M. Ali, A. Alqahtani, Mark W. Jones, and X. Xie. Clustering and classification for time series data in visual analytics: A survey. IEEE Access, 2019. [3] (Mohammed Ali and Ali Alqahtani contributed equally to this work).

4. A. Alqahtani, X. Xie, J. Deng, and Mark W. Jones. Learning discriminatory deep clustering models. International Conference on Computer Analysis of Images and Patterns (CAIP), 2019. [4]

5. A. Alqahtani, X. Xie, J. Deng, and Mark W. Jones. A deep convolutional auto-encoder with embedded clustering. IEEE International Conference on Image Processing (ICIP), 2018. [5] 


\section{List of Tables}

2.1 Summary of Modern CNNs and their performanc. . . . . . . . . . . . . . 46

3.1 Details of datasets used in our experiments for the DeepCluster method. . . . . . . 63

3.2 Detailed configuration of the DCAE network architecture used in the experiments. . 64

3.3 Comparison of clustering quality with the baselines on three datasets using two evaluation metrics: accuracy (ACC) and normalized mutual information (NMI). . . 67

4.1 Detailed configuration of the DCAE network architecture used in the experiments. . 80

4.2 Details of the datasets used in our experiments for the discriminatory DeepCluster models. . . . . . . . . . . . . . . . . . . . . . . . 84

4.3 Comparison of clustering quality of the DeepCluster method using accuracy evaluation metric with and without regularizations. . . . . . . . . . . . . 85

4.4 Number of samples used in the training stage and clustering accuracy. . . . . . . . 88

4.5 Comparison of clustering accuracy on four different datasets using different learning levels. . . . . . . . . . . . . . . . . . . . . . . . . 9 90

4.6 Supervised Clustering. Clustering accuracy with GBAR regularization technique using different weighting coefficients. . . . . . . . . . . . . . . . . . 92

4.7 Semi-supervised. Clustering accuracy with GBAR regularization technique using different weighting coefficients. . . . . . . . . . . . . . . . . 93

5.1 Details of Datasets and their FC Architectures used in our experiments. . . . . . 103

5.2 Examining neuron importance via ablation study with different selection criteria on CIFAR10. . . . . . . . . . . . . . . . . . . . . . . . 106

5.3 Examining neuron importance via ablation study with different selection criteria on MNIST. . . . . . . . . . . . . . . . . . . . . . . . 106

5.4 Summarization of our experiments with fully-connected networks. . . . . . . . . 107 
5.5 Summarization of our experiments with sparsely-connected networks. . . . . . . . 109

6.1 VGG-16 on CIFAR-10 and three different pruned models. . . . . . . . . . . . . 129

6.2 Performance of pruning VGG16 on CIFAR-10 . . . . . . . . . . . . . . . . . 130

6.3 Performance of pruning ResNet-20/32 on CIFAR-10 . . . . . . . . . . . . . . . 132

6.4 Performance of pruning ResNet-20/32 on CUB-200 . . . . . . . . . . . . . . 134

6.5 Performance of pruning ResNet-50 on ImageNet. . . . . . . . . . . . . . . 135

6.6 Comparison among several state-of-the-art pruning methods on ResNet-50 and ImageNet. . . . . . . . . . . . . . . . . . . . . 136

6.7 Overall results of layer-wise pruning utilizing different filter selection criteria for VGG-16 on CIFAR-10. . . . . . . . . . . . . . . . . . . . . . . . . 141

6.8 Overall results of layer-wise pruning utilizing different filter selection criteria for our small CNN model on CIFAR-10 . . . . . . . . . . . . . . . . . . . . . . . 141

6.9 Comparison of fine-tuning $(\mathrm{FT})$ and kernels estimation $(\mathrm{KE}) \ldots \ldots$

7.1 Deep Clustering Methods . . . . . . . . . . . . . . . . . . . . . . . . . . . . 158

7.2 Detailed configuration of the DCAE network architecture used on time-series data. 161

7.3 Experimental results of clustering quality, reporting averaged performance across 5 -fold cross-validation on three methods on the ICBD. . . . . . . . . . . . . . 164

7.4 Deep Time-series Clustering Methods. . . . . . . . . . . . . . . . . . . . . . . . . . 169 


\section{List of Figures}

2.1 Perceptron function process. . . . . . . . . . . . . . . . . . . . . 14

2.2 An overview of the two-layer network . . . . . . . . . . . . . . . . . 15

2.3 Visualization of common activation functions used in neural networks. . . . . . . . 19

2.4 Two main layers of CNNs. . . . . . . . . . . . . . . . . . . . . 22

2.5 Structure of the Inception and ResNet blocks. . . . . . . . . . . . . . . . . 26

2.6 The development of CNN Architectures. . . . . . . . . . . . . . . . . . . . 27

2.7 An Auto-encoder (AE) framework. . . . . . . . . . . . . . . . . . . . . . . . . . . 29

3.1 The architecture of the DeepCluster model. . . . . . . . . . . . . . . . . 64

3.3 Visualizations of reconstruction images (Top) and input images (Bottom) on 3 datasets using the DCAE-Kmeans proposed method. . . . . . . . . . . . . . . 69

3.4 Visualizing the reconstruction of clustering centres on 3 different datasets. Top: USPS; Middle: MNIST-FASHION; Bottom: MNIST. . . . . . . . . . . . . . . . 70

3.5 Visualizations of latent representations in a two-dimensional space with t-SNE on

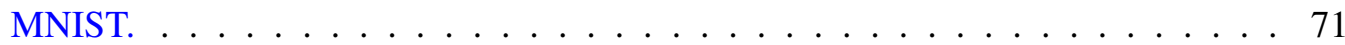

3.6 Visualizations of latent representations with our clustering results in a twodimensional space with t-SNE through an iterative training scheme on MNIST. . . 72

4.1 The architecture of the discriminatory DeepCluster models. An extra layer is added at the end of the network just after the reconstruction layer to inject different degrees of supervision. . . . . . . . . . . . . . . . . . . . 83

4.2 Visualizations of reconstruction images (Top) and input images (Bottom) in SVHN dataset. . . . . . . . . . . . . . . . . . . . . . . . 85

4.3 Visualizations of latent representation for our deep clustering method through through different levels of supervision on the MNIST testing set. Top: True Labels. Bottom: Our Results . . . . . . . . . . . . . . . . . . . . . . . . . . . . . . 89 
4.4 Invariance properties of the learned representation in different layers from five different models . . . . . . . . . . . . . . . . . . . . 91

5.1 Neuron-based pruning method. . . . . . . . . . . . . . . . . . . . . . . . 100

5.2 Majority voting (MV) method. . . . . . . . . . . . . . . . . . . . . 102

5.3 The architecture of the CNN model. . . . . . . . . . . . . . . . 108

5.4 Change in accuracy during training with our pruning method for three different models. . . . . . . . . . . . . . . . . . . . . . . 110

6.1 The overall pipeline of our proposed framework for pruning CNN filters via quantifying the importance of deep visual representations. . . . . . . . . . . . 118

6.2 The detailed process of our pruning procedure. . . . . . . . . . . . . . . . . 119

6.3 Scoring Channel Importance Method. . . . . . . . . . . . . . . . . . . . . . 121

6.4 Majority Voting (MV) method. . . . . . . . . . . . . . . . . . . . . . . 122

6.5 Different settings to determine the top quantile level $T$ in Eq.(6.2) at different layers of VGG16 on CIFAR-10 . . . . . . . . . . . . . . . 126

6.6 Different settings to determine the optimal $l$ value in Eq.(6.3) at different layers of VGG16 on CIFAR-10. . . . . . . . . . . . . . . . . . . . . . 127

6.7 Comparison of $I o U$ pruning selection criteria. . . . . . . . . . . . . . . . 128

6.8 Layer-wise pruning of VGG-16 on CIFAR-10 . . . . . . . . . . . . . . . . 130

6.9 Layer-wise pruning of ResNet-20/32 on CIFAR-10 . . . . . . . . . . . . . . . 131

6.10 Layer-wise pruning of ResNet 20/32 on CUB-200. . . . . . . . . . . . . . . 133

6.11 Different input images from the ImageNet dataset and their semantic segmentation and visualization of feature maps binary segmentation . . . . . . . . . 137

6.12 The architecture of the CNN model. . . . . . . . . . . . . . . . . . 139

6.13 Comparison of layer-wise pruning methods for VGG-16 on CIFAR-10 . . . . . . 140

7.1 The pipeline of conventional time-series analysis. . . . . . . . . . . . . 151

7.2 Line chart of the raw accelerometer data (Multivariate time-series data) . . . . . . . 159

7.3 The DAE network architecture for time-series data shown the number of neurons for each fully-connected layer in both encoder and decoder parts. . . . . . . . . 160

7.4 The detailed process of the sliding window approach with a window size of 12 and and a stride of 6 are adopted in both cases. . . . . . . . . . . . . . . . . 163

7.5 The pipelines of deep time-series clustering. . . . . . . . . . . . . . 166 


\section{Chapter 1}

\section{Introduction}

\section{Contents}

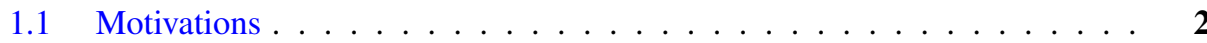

1.1.1 Deep Clustering . . . . . . . . . . . . . . . . . . . . 3

1.1.2 Learning Discriminatory Deep Clustering Models . . . . . . . . 4

1.1.3 Deep Neural Network Compression and Acceleration . . . . . . 4

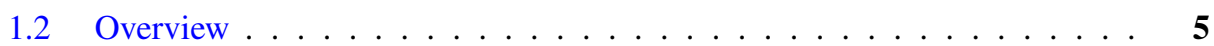

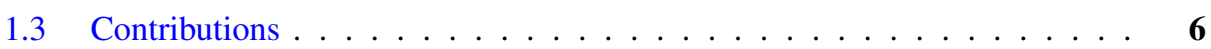

1.4 Outline $\ldots \ldots \ldots \ldots \ldots \ldots \ldots \ldots \ldots \ldots \ldots$ 


\subsection{Motivations}

Over recent decades, machine learning has rapidly grown as a tool for analyzing and utilizing data, presenting a wide range of methodologies to extract information from observed data. Different approaches have been developed to understand the characteristics of the data and obtain meaningful statistics in order to explore the underlying processes, identify and estimate trends, make decisions and predict the future. Driven by a justifiable belief that an improved feature-extraction pipeline and a cleaner and bigger dataset are entirely mattered to the final performance [6], the use of hand-crafted features to represent data structures has been replaced, shifting the focus to representation learning and features extraction and encouraging the improvement of automated learning techniques which are able to optimize their feature extractors and learn representations from observed data. Deep learning methods have proven robust in representation learning and have grown increasingly widespread in recent years. As a result of the greater availability of data and advanced computing power, deep learning has advanced into wider and deeper models, driving state-of-the-art performance across various tasks, especially in computer vision (i.e., object detection [7], semantic segmentation, [8] and image classification [9-11]). This achievement has been possible through the ability of deep learning to discover, learn, and perform automatic representation by transforming raw data into an abstract representation, which allows the system to learn the right representations from the raw data. During the learning process, a deep learning model utilizes a hierarchical level of neural networks and can learn feature representations at multiple abstraction levels so complicated concepts can be developed from simpler ones.

The development of the deep learning strategy for representation learning relies heavily on the choice of data representations (or features) and the improvement of the feature-extraction pipeline [6]. Therefore, much of the effort in developing, exploring, or analyzing deep learning algorithms go into the structure underlying discriminative and representative features, and the ability to learn the identification and disentanglement of the underlying explanatory factors hidden in the data, in order to expand the scope and ease of applicability of deep learning models [12].

The overarching motivation of this thesis is to continue the current trend in making use of learned representations, specifically to enhance unsupervised learning and evaluate the characteristics of internal representations to compress and accelerate deep neural networks. The use of representation learning for the clustering process will be explored, and an in-depth analysis of strengthening the discriminative features in relation to improvements in the performance 
of deep clustering methods will be provided. The effectiveness of deep representations will be investigated by measuring their importance, where a novel pruning framework is presented based on quantifying the importance of deep visual representations of Convolutional neural networks (CNNs).

\subsubsection{Deep Clustering}

Clustering is a fundamental task in a number of areas, including machine learning, computer vision, and pattern recognition [13]. The goal of clustering is to group a set of unlabeled data in the given feature space based on similarity measures (e.g. Euclidean distance). Although conventional clustering methods have received significant attention [14-16], they attain limited performance as dimensionality increases, and usually suffer from high computational complexity on large-scale datasets. To overcome the weaknesses associated with high dimensionality, many approaches corresponding to dimensionality reduction and feature transformation methods have been extensively studied, including linear mapping (i.e. principal component analysis (PCA) [17]), non-linear mapping (i.e. kernel methods [18] and spectral methods [19]). Nevertheless, a higher-level, more complex latent structure of data still challenges the effectiveness of existing clustering methods [20]. Due to the development of deep learning [21], deep neural networks minimize this issue by allowing a clustering algorithm to deal with clustering-friendly features, as working with high-level representations provides beneficial components that support the achievement of traditional algorithms to demonstrate satisfactory performance. As there is no supervision knowledge to provide information on categorical labels, representative features with compact clusters are more valuable. They allow a clustering algorithm to obtain characteristic features and extract useful information for its structure. Chapter 2 introduces the current state of deep clustering approaches and highlights the need to develop an unsupervised deep learning method that enables on to embed a clustering approach into a deep network more appropriate to image processing tasks. Such joint optimization often leads to a more compact hidden feature space and minimizes the time cost needed in multi-step deep clustering methods [13]. Chapters 3, 4, and 7 are motivated by this aim to explore the utilization of deep learning methods for the clustering process, identifying a feature representation that can compute informative features, spatially localized, on the input space, and support the achievement of deep clustering methods in the latent space. An evaluation is carried out using numerous datasets and a case study. 


\subsubsection{Learning Discriminatory Deep Clustering Models}

The work on deep clustering contains novel materials and it has become crucial to explore and extend this with further analysis. In the procedure of a deep clustering method, the discriminative patterns are only discovered through certain parts or patterns in a data sample in an unsupervised manner, with limited attention paid toward enhancing more discriminative latent features to further support the embedded clustering. In an attempt to investigate the ways in which to reinforce the performance of conventional clustering methods, several methods have been developed to study semi-supervised clustering and supervised clustering approaches. Despite the substantial success of deep learning, there has been limited focus on deep supervised and semi-supervised clustering models; to address this, Chapter 4 provides an analytical study for understanding the effectiveness of differing discriminatory power, focusing on strengthening and discriminating the learned features. Such a mechanism would ensure that the learned features derived from the encoding layer are the best discriminative attributes by reconciling the ability of representation learning and discriminative powers imposed on the clustering layer or injected into the body of the learning process. Evaluation is provided using four different datasets, considering several regularization techniques, through varying degrees of supervision.

\subsubsection{Deep Neural Network Compression and Acceleration}

Despite the success of deep learning models, deep networks often possess a vast number of parameters, and their significant redundancy in parameterization has become a widely-recognized property [22]. The over-parametrized and redundant nature of deep networks results in expensive computational costs and high storage requirements - significant challenges which restrict many deep network applications. Network pruning focuses on discarding the unnecessary parts of neural networks, aiming to obtain a sub-network with fewer parameters without reducing accuracy. The pruned version, a subset of the whole model, can represent the reference model at a smaller size or with far fewer parameters. Reducing the complexity of models while maintaining their high performance creates unprecedented opportunities for researchers to tackle the challenges of deploying deep learning systems to a resource-limited device and increase the applicability of deep network models to a broader range of applications. While pruning approaches have received considerable attention as a way to tackle over-parameterization and redundancy, some existing methods require a particular software/hardware accelerator that is not supported by off-the-shelf libraries. The random connectivity of non-structured sparse models can also cause poor cache locality and jumping memory access, which significantly limits the 
practical acceleration [23]. Moreover, most existing methods tend to focus on applying simple pruning techniques (e.g. statistical approaches) to compress networks rather than discovering informative units for effective pruning [24]. To confront these challenges, Chapter 5 and Chapter 6 introduce pruning frameworks, where removing an unimportant part in its entirety does not change the network structure contrary to previous methods, and any off-the-shelf deep learning library can support the method. This procedure can also effectively reduce memory requirements as model compression focuses on reducing not only model parameters but also the intermediate activation. The ways in which to integrate the pruning procedure into the learning process are investigated in Chapter 5 to allow the finding of a smaller architecture to the target task at the training phase, which avoids the need for a multi-step pruning procedure. Unlike other pruning methods, the training-based pruning method allows the input configuration to be handled by applying a constraint function to the weights matrix during training without changing the network structure and adaptively determining hyperparameters. The proposed method is evaluated across various scenarios, including fully-connected networks (FCNs), sparselyconnected networks (SCNs), and CNNs using two datasets. Chapter 6 explores the concept that not all filters deliver essential information for the final prediction of the model [25-27] and fundamentally relies on quantifying the importance of latent representations of CNNs by evaluating the alignment/matching between semantic concepts and individual hidden units to score the semantics of hidden units at each intermediate convolutional layer. The core aim is to evaluate filters' importance, which provides meaningful insight into the characteristics of neural networks' internal representations, reducing the computational complexity of the convolutional layers. The pruning method is evaluated on large-scale datasets and well-known CNN architectures.

\subsection{Overview}

In line with the rationale presented in section 1.1, this study aims to explore the use of representation learning in deep clustering and deep network compression. In previous years, common approaches for deep clustering have focused on taking advantage of a deep neural network, separating the learning process from the clustering task, which requires various emphases and involves a cumbersome, time-consuming process. Chapter 3 introduces the use of DeepCluster, an embedded clustering approach in a deep convolutional auto-encoder (DCAE) for efficient simultaneous, end-to-end learned local features and cluster assignments. This scheme usually leads to a more compact latent feature space and enables a faster process. Chapter 4 utilizes 


\section{Introduction}

the DeepCluster to explore the use of deep clustering carried out in presence of varying degrees of discriminative power by evolving a mechanism to inject various levels of supervision into the learning process or impose constraints on the clustering layer. In Chapters 5 and 6, overparameterized networks are efficiently compressed and allow for the acquisition of a small subset of the whole model, representing the reference model with much fewer parameters. This work explores the use of representation learning in deep network compression, presenting two pruning frameworks for neurons in fully-connected layers and filters in convolutional layers.

\subsection{Contributions}

The main contributions of this thesis are as follows:

\section{- A deep convolutional auto-encoder with embedded clustering.}

We propose DeepCluster, a clustering approach embedded into a deep convolutional auto-encoder (DCAE) framework which can alternately learn feature representation and cluster assignments. In contrast to conventional clustering approaches, our method makes use of representation learning by deep neural networks, which assists in finding compact and representative latent features for further recognition tasks. It also exploits the strength of DCAE to learn useful properties of image data for the purpose of clustering. In this work, we introduce two deep clustering methods: DCAE-Kmeans and DCAE-GMM. An objective function that reduces the distance between learned feature representations in a latent space and their identical centroids is applied. A mixture of multiple Gaussian distributions in a latent space is also considered, so that all data samples are assumed to be generated from multiple Gaussian distributions. These procedures enable the classification of a data point into its identical cluster in the latent space in a joint-cost function by alternately optimizing the clustering objective and the DCAE objective, thereby producing stable representations appropriate for the clustering process. The proposed method is trained in an end-to-end way using fixed settings without any pre-training or fine-tuning procedures, enabling a faster training process. Qualitative and quantitative evaluations of proposed methods are reported, showing the efficiency of deep clustering on several public datasets in comparison to previous state-of-the-art methods. This work was published in [5]. 
- Learning discriminatory features when searching for a compact structure to form robust clusters.

We present a new version of the DeepCluster to include varying degrees of discriminative power. This work introduces a mechanism to allow for the imposition of regularization techniques and the involvement of a supervision component; effectively reconciling the extracted latent representations and the provided supervising knowledge to produce the best discriminative attributes. The key idea of our approach is distinguishing the discriminatory power of numerous structures when searching for a compact structure to form robust clusters. The effectiveness of injecting various levels of discriminatory powers into the learning process is investigated alongside exploration and analytical study of the discriminatory power obtained through the use of several discriminative attributes. Two regularization techniques are considered: one that is embedded in the clustering layer and another that is used during the training process. We also take into account three different learning levels: supervised, semi-supervised and unsupervised. An evaluation is provided using four different datasets. This work was published in [4].

\section{- An iterative pruning method to reduce network complexity.}

We propose an iterative pruning method that prunes neurons based on the level of importance during training, without involving pre-training or fine-tuning procedures. The proposed method mainly targets the parameters of the fully-connected layers, does not require special initialization, and can be supported by any off-the-shelf machine learning library. We introduce a majority voting technique, which aims to compute a measure of relevance that identifies the most critical neurons by assigning a voting score to evaluate their importance and helps to effectively reduce model complexity by eliminating less influential neurons, with the aim of determining a subset of the whole model which can represent the reference model with fewer parameters within the training process. A comparative study with experimental results on public datasets is presented. An evaluation is also provided across various scenarios, including FCNs, SCNs, and CNNs. This work was published in [2].

\section{- Pruning CNN filters via quantifying the importance of deep visual representations.}

We propose a novel framework to measure the importance of individual hidden units by computing a measure of relevance to identify the most critical filters and prune them to compress and accelerate CNNs. This work is considered pioneering in that it attempts to 
use the quantifying interpretability for more robust and effective CNN pruning. Inspired by novel neural network interpretability, we first introduce the use of the activation of feature maps as well as the use of and essential semantic parts to detect valuable information and evaluate the importance of feature maps. A majority voting technique based on the degree of alignment between a semantic concept and individual hidden unit representations is proposed to quantitatively evaluate the importance of feature maps, as well as a simple, effective method to estimate new convolution kernels based on the remaining crucial channels to recover compromised accuracy. An evaluation is provided using large-scale datasets and well-known CNN architectures. A comparison with other state-of-the-art pruning methods is given. A paper [1] has been submitted and is under review at the time of writing.

\section{- Deep embedded clustering of time-series data for movement behavior analysis.}

We present a comprehensive, detailed review of time-series data analysis, with emphasis on deep time-series clustering (DTSC), and a case study in the context of movement behavior clustering utilizing the DeepCluster method. Specifically, we modified the DCAE architectures to suit time-series data; see Chapter 7 for details. The work was done in 2017, and we believe that we were the first to approach this topic and have made founding contributions to the area of deep clustering of time-series data; Chapter 7 describes these contributions. Since 2018, several works have been carried out on DTSC. We also review these works and identify state-of-the-art and present an outlook on this important field of DTSC. A part of this work was published in [3]. 


\subsection{Outline}

The remaining chapters of this work are outlined as follows:

Chapter 2 Background:

The necessary background information surrounding neural networks and deep learning, as well as an overview of popular methods and considerations required in deep clustering and deep network compression and acceleration.

Chapter 3 DeepCluster: A Deep Convolutional Auto-encoder with Embedded Clustering: Introduces clustering approaches embedded into a deep convolutional auto-encoder (DCAE). Two joint-cost functions are introduced, where the clustering objective and DCAE objective are alternately optimized. Experimental evaluations of the method are presented in comparison to previous state-of-the-art methods.

Chapter 4 Learning Discriminatory Deep Clustering Models:

Explores the use of DeepCluster to study discriminative power when searching for a compact structure to form robust clusters in a latent space in presence of regularization techniques and supervision components. Experimental evaluations of the method are presented and discussed.

Chapter 5 Reducing Neural Network Parameters via Neuron-based Iterative Pruning:

The proposed iterative pruning method prunes neurons to reduce network complexity, an activation-based method which iteratively prunes neurons based on a measure of relevance that identifies the most critical neurons by assigning a voting score to evaluate their importance. Experimental evaluation and analytical discussions are provided.

Chapter 6 Pruning CNN Filters via Quantifying the Importance of Deep Visual Representations:

Introduces a novel framework to measure the importance of individual hidden units by evaluating the degree of alignment between a semantic concept and individual hidden unit representations. A majority voting technique is proposed to quantitatively evaluate the importance of feature maps. The performance of the method is evaluated and compared with other state-of-the-art pruning methods. 


\section{Chapter 7 Deep Time-Series Clustering:}

Presents a comprehensive, detailed review of time-series data analysis, introducing the use of DeepCluster methodology to learn and cluster temporal features from accelerometer data for the clustering of animal behaviors. An evaluation and discussion are given on real-world data, namely, the Imperial Cormorant bird dataset (ICBD). The state-ofthe-art and an outlook of DTSC are also provided.

\section{Chapter 8 Conclusions and Future Work:}

Concluding remarks on studies presented in the previous chapters, highlighting opportunities and potential future directions. 


\section{Chapter 2}

\section{Background}

\section{Contents}

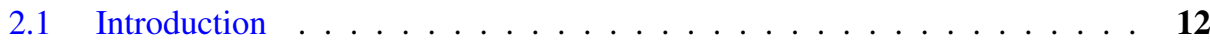

2.2 Deep Learning . . . . . . . . . . . . . . . . . . . . . . 13

2.2.1 Neural Networks . . . . . . . . . . . . . . . . . . . 13

2.2.2 Convolutional Neural Networks . . . . . . . . . . . 21

2.2.3 Modern Convolutional Neural Networks . . . . . . . . . . . . . . 24

2.2.4 Auto-encoders ...................... 28

2.2.5 Advances in Deep Learning . . . . . . . . . . . . . . 30

2.2.6 Visualizing, Explaining and Interpreting Deep Learning . . . . . . 31

2.3 Clustering ....................... 34

2.3.1 Conventional Clustering Methods . . . . . . . . . . . . . 34

2.3.2 Deep Clustering Methods . . . . . . . . . . . . . . 38

2.3.3 Clustering Supervision . . . . . . . . . . . . . . 44

2.4 Deep Neural Network Compression and Acceleration _. . . . . . . 45

2.4.1 Pruning Methods . . . . . . . . . . . . . . . 47

2.4.2 Quantization Methods ............... 51

2.4.3 Low-rank Factorization Methods . . . . . . . . . . . . . . 52

2.5 Summary ....................... 53 


\subsection{Introduction}

Over recent decades, machine learning has rapidly grown as a tool for analyzing and utilizing data, presenting a wide range of methodologies to extract information from observed data $[28,29]$. Machine learning gives computers the ability to learn without explicit programming [30]. Alpaydin [31] gives a concise description of machine learning, which is "optimizing a performance criterion using example data and past experience". Machine learning algorithms provide a collection of automated analyses that can be much more efficient, accurate, and objective in solving different tasks. Data plays a significant role in machine learning, where the learning algorithm is utilized to discover and learn knowledge or properties from the data (learn from experience) without depending on a predetermined equation as a model [32]. In supervised learning, the training set is composed of pairs of input and desired output, and the learning aim is to generate a function that maps inputs to outputs. Each example is associated with a label or target. In unsupervised learning, the training set is composed of unlabeled inputs without any assigned desired output, and the aim is to find hidden patterns or substantial structures in data [33].

The dependence on hand-crafted features over raw data is a general phenomenon that appears as a standard procedure for most machine learning algorithms [6]. However, a careful approach is required by domain experts in order to develop informative features and generalize information across the distribution of observations. For instance, Histogram of Oriented Gradients (HoG) [34] and Scale Invariant Feature Transform (SIFT) [35] are feature descriptors which are generalized well to image domain problems and developed from a knowledge of image processing principles. Feature descriptors would allow the classical machine learning methods to deal with features instead of raw data and train to recognize patterns in a feature space. Notwithstanding, the evolution of the deep learning strategy replaces the need for handcrafted feature descriptors by enabling a model to learn its extractor set from the data in a new representation space [12]. This process is referred to as the term of "representation learning", which has rapidly grown in the last decade with the development of neural networks and deep learning [21].

In recent years, deep learning has rapidly grown and begun to show its robust ability in representation learning, achieving remarkable success in diverse applications. This achievement has been possible through its ability to discover, learn, and perform automatic representation by transforming raw data into an abstract representation. The process of deep learning utilizes a hierarchical level of neural networks of different kinds, such as multilayer perceptron (MLP), 
convolutional neural networks (CNNs), and recurrent neural networks (RNNs). This hierarchical representation allows models to learn features at multiple abstraction levels, meaning that complicated concepts can be learned from simpler ones. Neurons in earlier layers of a network learn low-level features, while neurons in later layers learn more complex concepts [32].

This chapter presents an overview of deep learning algorithms and the relevant background knowledge considered in this thesis. An introduction to deep neural networks is discussed in section 2.2. First, we walk through the basic operations, learning procedure, and optimization of deep neural networks. Then, we provide insight into CNNs, discussing the backbone of all convolutional neural networks and the structure of modern architectures along with their motivations, advantages, and disadvantages. Later, we provide a review of auto-encoder (AE) and other advanced deep learning algorithms. The development of visual interpretability to understand deep networks is also discussed. In section 2.3, an overview of conventional clustering methods is presented. Following this, we provide a review of deep clustering methods, introducing different approaches to said methods and their fundamental structure. Thereafter, in section 2.4, we present an overview of popular methods for compressing and accelerating deep neural networks, reviewing the recent works of related techniques used in our research. The focus is essentially on deep clustering and deep network compression, as they form the primary subjects of this thesis. Finally, a concluding summary is provided in section 2.5.

\subsection{Deep Learning}

\subsubsection{Neural Networks}

Inspired by neurobiology, neural networks (NNs) grew by way of artifical neurons as the precursor of perceptron from the combination of input stimuli and the activation response of interconnected neurons in the human brain [36]. The algorithm for NNs is a machine learning technique that consists of simple processing elements known as units, neurons or nodes, which are linked together with weighted connections acquired by a process of learning from a set of training data [37]. A perceptron, considered to be the foundation of all neural network models, is a building block in NN structure which presents a fundamental mathematical model of a neuron adopted in all neural network models. Fig. 2.1 shows the mathematical model of a single neuron (perceptron), which consists of input values $X$, weights $W$ and bias $b$, a summation operation, an activation function and an output. The perceptron was introduced as a function which computes a weighted summation of all inputs, adds a bias and passes it through 


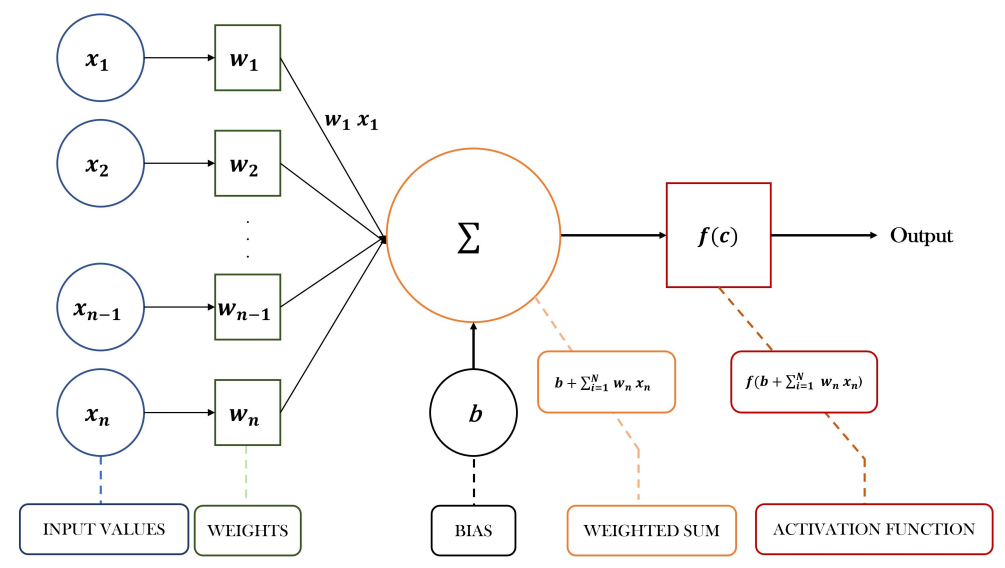

Figure 2.1: Perceptron function process. The output $c$ is calculated as the weighted sum of the input $x$ added to a bias value $b$, which is then passed through a non-linear activation function $f(c)$.

an activation function to determine the output $[36,38,39]$. The obtained output is the overall output from the perceptron, mapping the input into a new feature representation.

Given an input vector $x=\left(x_{1}, x_{2}, \ldots, x_{n}\right)$, the output $c$ is computed as the weighted sum of the input given by using the following form:

$$
c=b+\sum_{i=1}^{N} x_{i} w_{i},
$$

where $b$ is the learned bias for the perceptron, and $w_{i}$ is the learned weight for the input $x_{i}$. The $c$ output can be then passed through a non-linear activation function $f(c)$ to produce the final perceptron's output (Fig. 2.1). Learning the perceptron's parameters is a linear optimization problem. The perceptron is utilized to compute the outputs for a set of inputs, and the determined outcomes are then compared with ground truth results, returning an error known as loss function which is used to update the parameters of perceptron. This procedure is iteratively performed with the new weights until minimizing the error. For higher-dimensional data, a group of perceptrons can be consolidated into a single-layer perceptron or extended to MLPs to learn feature representations at multiple levels of abstraction.

\subsubsection{Multilayer Perceptrons (MLPs) and Feed-forward Networks}

The necessity to simultaneously learn multiple functions and increase the capacity of learning representations in solving non-linear problems motivated Rosenblatt to introduce stacked layers of perceptrons [36], inspired by neuroscience which found that neurons firing in the brain are connected to other neurons [40]. The output of a neuron is passed as the input to a sub- 


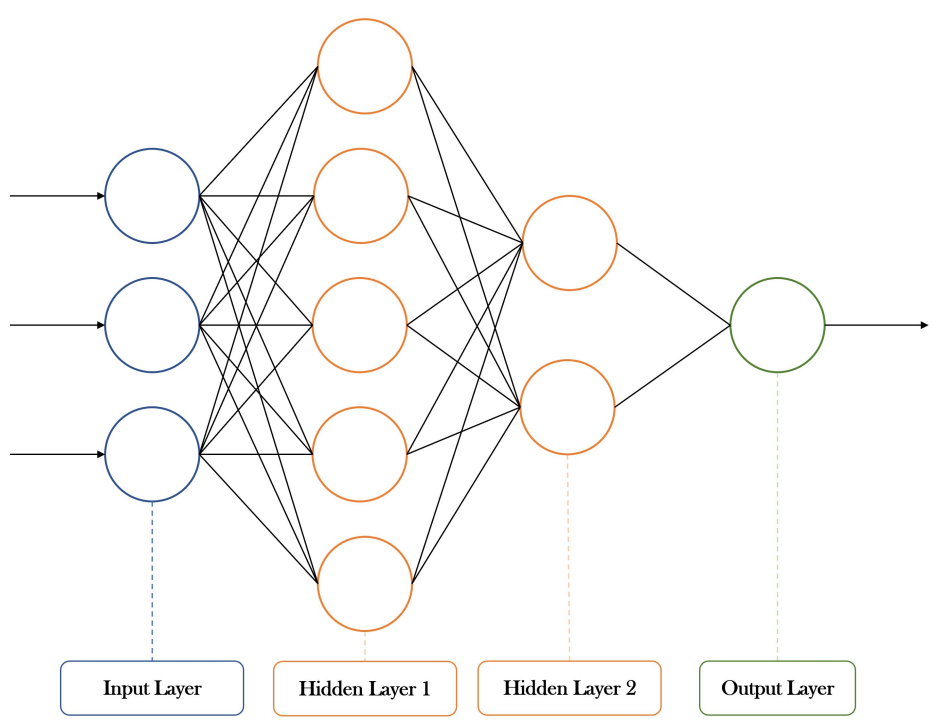

Figure 2.2: An overview of the two-layer network. Each hidden layer consists of one or more perceptron, and each perceptron has its own trainable weights and bias as well as a non-linear activation function.

sequent neuron in the next layer of the network. From this notion, the architecture of MLPs is made up of a sequential structure of multiple layers (Fig. 2.2), with each layer containing neurons with weighted interconnections between them [41], forming a fully connected neural network. Neurons act as switching units associated with interconnected weights, with the aim of ideally approximating function (e.g. classifier function) by mapping the input values into a category (a class) to learn the parameters (weights) [32]. With the notion of multiple layers of a sequential network, Eq.(2.1) is generalized to the following form:

$$
c_{j}^{l+1}=b_{j}^{l+1}+\sum_{i=1}^{N} x_{i}^{l} w_{i j}^{l},
$$

where $b_{j}^{l+1}$ denotes the corresponding bias for the $j$-th neuron in the $l+1$-th layer, $x_{i}$ denotes the input value of a perceptron $i$, and $w_{i j}^{l}$ is the weight that connects $i$-th neuron from the previous layer $l$ with the $j$-th neuron in the $l+1$-th layer (existing layer). Like standard Eq.(2.1), the linear transformation of the perceptron $c_{j}^{l+1}$ is passed to a non-linear activation function. Each internal layer (hidden layer) processes the input data as per the activation function and passes it to the successive layer. This hierarchical process allows the MLP to learn better representations of the input data at multiple levels of abstraction and via non-linear mapping [21]. Larger perceptron counts in the hidden layers increase the information processing capacity and allow the layer to better represent the input features, but struggles to enable higher-level generalization. 


\section{Background}

However, using too few layers and nodes in layers can lead to underfitting the input data while using too many layers and nodes in layers can lead to overfitting the input data [11,42]. Underfitting arises when the information processing capacity is not sufficient to detect the signals in a complex data set, while overfitting occurs when the information processing capacity of the neural network is far more than the amount of information contained in the training set. A balance between the two makes architecture design (i.e. the number of perceptrons and layers, activation functions, etc.) a sensitive task with which to achieve achieve generalization without overfitting. The overall architecture design of a neural network model is still an open research topic, with several research papers dedicated to investigating it [43-48].

\subsubsection{Back-propagation and Weight Optimization}

The back-propagation algorithm [49] is the core of neural network learning, where parameters are learned to reach the minimum cost function value, relying on the back-propagation of errors to optimize the parameters. The process of back-propagation refers to the method of optimizing the parameters of MLP or feed-forward network methods in order to learn the latent space function from the observed samples. Feeding the training data through the network, each example is represented differently and has individual predicted output. In supervised learning, class labels should be given, and the weights are learned by finding the best relationship between the input data and its appropriate classes. The error loss between the outputs computed by the current model and ground truth labels given by the training dataset is computed and back-propagated in reverse order, from the output to the input layer, based on the chain rule from calculus to calculate the derivative with regard to the network parameters. During the training process, weights are tweaked and changed by minimizing the loss function using an optimization method, such as gradient descent optimization. Feeding the training data $t$ through the network, each example has individual predicted output vector. The objective function calculates the difference between the predicted output vector $\alpha_{j}^{L}$ and the expected target value of $y_{j}$, where $j$ corresponds to a given class. In classification tasks, a famous example of a loss function is the cross-entropy, given by:

$$
E=-\frac{1}{t} \sum_{x} \sum_{j} y_{j} \operatorname{In} \alpha_{j}^{L}+\left(1-y_{j}\right) \operatorname{In}\left(1-\alpha_{j}^{L}\right)
$$

where $t$ is the total number of training examples, and $\mathrm{x}$ is a given training input. The cost function $E$, between the output sample and the input, is then back-propagated through the 
network to update individual weights. The partial derivative of each neuron is computed with respect to a given neuron's inputs and their weights:

$$
\Delta \omega_{i j}=-\alpha \frac{\partial E}{\partial \omega_{i j}}
$$

where $\omega_{i j}$ denotes the weights between two neurons $i$ and $j, \alpha$ is the learning rate used to adjust the feature weighting. The partial derivative is then added: $\omega_{i j}=\omega_{i j}+\Delta \omega_{i j}$ to update each weight. Here, we name a particular loss function and optimizer to provide an overview of the neural networks' background and their working mechanisms. However, there are many other choices for these roles in the neural networks, and selecting the right loss function and optimizer is critical to optimize the network parameters, depending on the task at hand [50]. With deep learning toolboxes, it has become more popular to attach a method of automatic differentiation to each function [51,52], allowing each layer to be used as a feed-forward operator, and the derivative with respect to the weights and biases to be obtained.

The effectiveness of the back-propagation becomes minor as the network goes deeper. When errors are back-propagated through each layer of the network, the derivatives are obtained for each neuron, and the gradients identified for use in stochastic gradient descent updates quickly depreciate [53]. This may be due to the use of certain activation functions (i.e. Sigmoid and Tanh) which suffer from diminishing gradient issues and cause a derivative to vanish quickly towards zero, which saturates the neurons in the layer and decreases their gradients. The saturation of the sigmoid function and vanishing gradient problem attain a limited optimization gain during training and affect the model's final performance [54]. In order to overcome back-propagation challenges and achieve more effective learning, several approaches have been introduced. The Rectified Linear Unit (ReLU) activation has become the most commonly used activation function, overcoming issues of all other activation functions (e.g. sigmoid and Tanh), as it speeds up neural network training and presents a stable derivative for all positive values. In practice, the use of regularization techniques (i.e. batch normalization [55] and dropout layers [56]) has proven very effective [57,58] in improving the generalization of deep network models.

Batch normalization [55] introduces a method to standardize the mean and variance within a layer based on the experimental batches during training. Thus, new observations at test time will be normalized based on the learned parameters. The batch normalization aids to stabilize learning, avoids gradient explosion, and enables models to be more generalized. Dropout [56] presents a method of preventing large networks from overfitting by randomly dropping a neu- 


\section{Background}

ron out based on which the income or outgoing connections are removed during training. This mechanism enables the model to be more generalized, aiming to stifle the co-adaptation of neurons. At test time, the dropout rate is set to 1, leaving the output unchanged for all network neurons.

As well as these, different settings of optimization algorithms and learning rate can aid the overall generalization [6]. Optimization algorithms have received considerable attention and several optimizers have been proposed as an extension to stochastic gradient descent [59]. Mini-batch gradient descent [60], Adagrad [61], Adadelta [62], and Adam [63] are the most advanced optimization algorithms for neural network training. They mimic many of the desirable properties of the stochastic gradient descent and also consolidate parameters for learning rate to influence the stability of the gradient steps that are made during parameter updates.

\subsubsection{Activation Functions}

The activation function is considered a mathematical gate between the input of the current neuron and its output going to the next layer. It can be a simple binary step function that turns the neuron output on and off based on a threshold, or a feature transformation that maps the original data into latent representation spaces required for the neural network to function. A linear activation function in a neural network model is simply a linear regression model. The role of a neural network with a non-linear activation function is to enable networks to reproduce more complex non-linear function spaces and solve the limitations of linear models. Several activation functions have been introduced and utilized in modern deep learning implementations, each of which has varying degrees of impact on the model's training and overall efficiency when dealing with different types of data. The types of activation function discussed below are intended to provide an overview of popular related techniques used in our research, not to define a taxonomy of functions.

A linear activation function is another simple function where an input passes to the output with no change (see Fig 2.3A.). The linear function is used with particular layers (i.e. the output of the encoding layer in the model of auto-encoders latent space). However, the backpropagation has no impact, as the derivative of this function is a constant and has no association with the input. Regardless of how many layers in the network, if all are linear, the last layer will be a linear function of the first.

$$
f(x)=x
$$




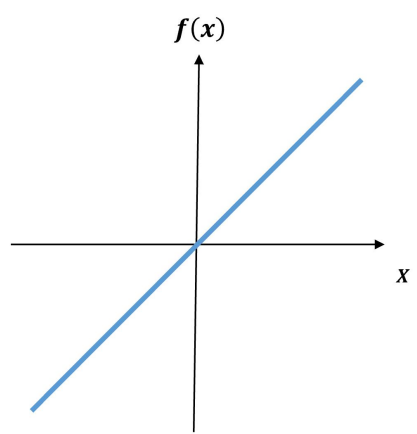

A. Liner

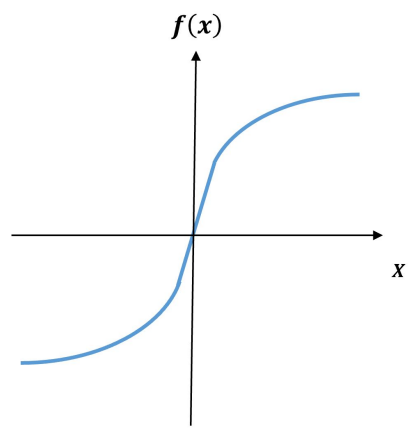

D. Tanh

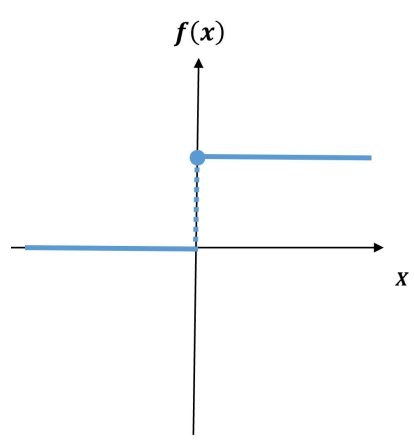

B. Binary Step

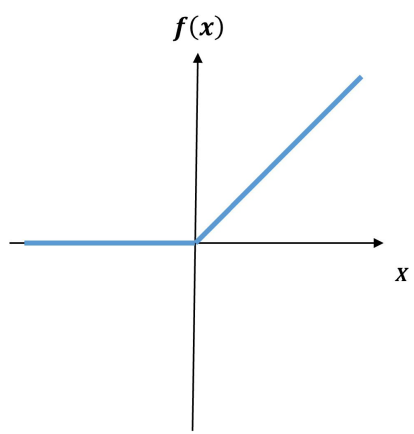

E. ReLU

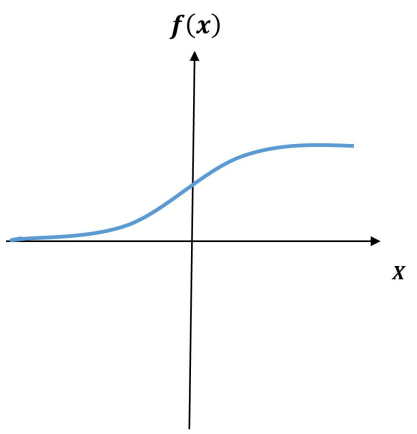

C. Sigmoid

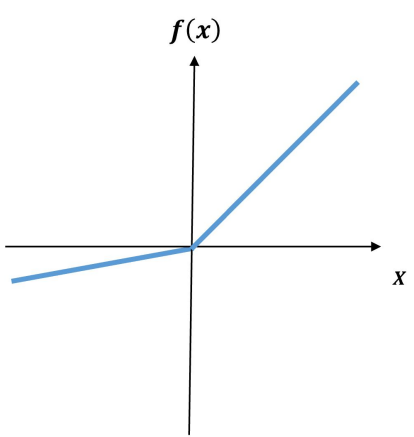

F. Leaky ReLU

Figure 2.3: Visualization of common activation functions used in neural networks.

The limitation of the ability and power of linear functions to deal with complex varying parameters of input data raises the necessity to employ continuous activation functions in their place.

A binary step function is the original activation of a perceptron. The perceptron is fired and sends the same signal to the next layer if the input value satisfies a certain threshold. This function indicates whether or not a neuron should be activated (see Fig. 2.3B.).

$$
f(x)=\left\{\begin{array}{ll}
1, & \text { if } x>0 \\
0, & \text { otherwise }
\end{array} .\right.
$$

The binary step function works well with linear binary classification tasks where a perceptron would estimate a line class boundary and the output predicts whether or not the data is part of a specific class. However, this activation function does not allow multi-value outputs and proves to be ineffective for feature mapping between layers of MLPs. A reasonable explanation 


\section{Background}

is that when data is passing between layers, thresholded outputs prevent learning of meaningful feature representations. In addition, the derivative of step function has an undefined gradient at zero, which means the back-propagation will fail as an optimizer and be unable to make progress in updating the weights.

The sigmoid activation function prevents jumps in output values through mapping input values between 0 and 1 (see Fig. 2.3C.) via the following equation:

$$
f(x)=\frac{1}{1+e^{-x}} .
$$

This simple activation function is commonly used for neural networks, allowing networks to learn and model complex data and represent complex mappings. However, it suffers from the saturation and vanishing gradient issues [53]. When back-propagating, minimal gradients are obtained for neurons whose outputs are close to 0 or 1, known as saturated neurons [54]. This prevents the network from learning further or reduces the speed at which a correct prediction is reached, which may explain the limited research of neural networks from 1990 until the rise of the Hyperbolic Tangent (Tanh) and ReLU functions.

The Tanh function provides a zero-centred activation to solve the saturation of the sigmoid function, making it more manageable to model inputs that have extreme positive, neutral, and negative values (see Fig. 2.3D.).

$$
f(x)=\frac{e^{2 x}-1}{e^{2 x}+1} .
$$

Although the Tanh function becomes the preferred choice over the sigmoid activation, it still demonstrates the vanishing gradient problem [64]. In recent years, the ReLU activation has proven useful in overcoming the previous activation issues, enhancing the convergence when training a model and allowing the network to converge quickly [65]. The ReLU activation similarly acts as a linear function with thresholding negative values to 0 , allowing the provision of constant gradients with positive inputs and zero gradients elsewhere (see Fig. 2.3E.). The constant gradient greatly reduces the vanishing gradient problem [64], ensuring the gradient descent algorithm does not stop learning as a result of a vanishing gradient.

$$
f(x)=\max (0, x) .
$$

Although the standard ReLU activation has received significant attention and become a popular activation function, it nevertheless presents issues. When inputs are negative or ap- 
proach zero, the gradient of the ReLU activation function becomes zero, driving weight optimization to result in dead neurons which never fire. These dead neurons do not contribute to the final model performance, essentially rendering them trivial and non-informative to the network. In order to prevent the dying ReLU issue, residual connections described later in this chapter have offered a popular idea to maintain the magnitude of the gradients. Moreover, several improvements to ReLU have been introduced to mitigate this without losing the advantages of the ReLU function. Leaky Rectified Linear Unit (Leaky ReLU) is considered one of the most popular alternatives [66], given by the following form:

$$
f(x)=\left\{\begin{array}{cc}
x, & \text { if } x \geqslant 0 \\
\alpha x, & \text { otherwise }
\end{array}\right.
$$

Leaky ReLU allows a small positive slope in the negative area by $\alpha \in[0,1]$, instead of a thresholded negative value to 0 (see Fig. 2.3F.). This function enables back-propagation and solves the problem of dead neurons. Thanks to recent studies and the development of linear unit functions, deep neural networks have advanced in various tasks, driving these activation functions to be the standard option for the majority of neural network models [57, 67].

\subsubsection{Convolutional Neural Networks}

The evolution of deep learning algorithms for learning representation coincided with the emergence of Convolutional Neural Networks (CNNs) [68]. CNNs are introduced as a kind of neural network that has been developed for processing data with a grid-structured topology, such as time-series (1-D grid) data and image data (2-D grid of pixels). In contrast to standard architectures of NNs, CNNs comprise convolutional layers for spatially-related feature extraction, so instead of matrix multiplication, convolutional layers apply a mathematical operation called convolution that slides a locally connected filter consisting of trainable weights through parts of the input to learn localized information from different regions of the input. This procedure efficiently reduces the number of trainable weights and allows CNNs to admit multi-dimensional arrays of traditional data (e.g. images) as an input, instead of an arbitrary feature vector. The convolutional part generally consists of multiple stages, and each layer has three stages: the convolution stage (filter), the detector stage (activation) and the pooling stage [32]. The input and output of each stage are called feature maps [69]. The convolutional layer and the pooling layer are two new building blocks introduced with the advent of CNN (see Fig. 2.4). 


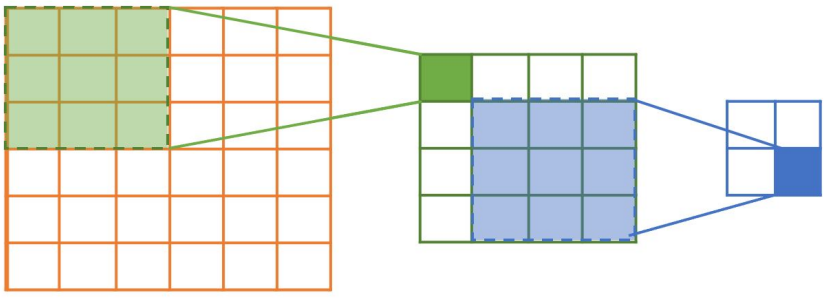

A.

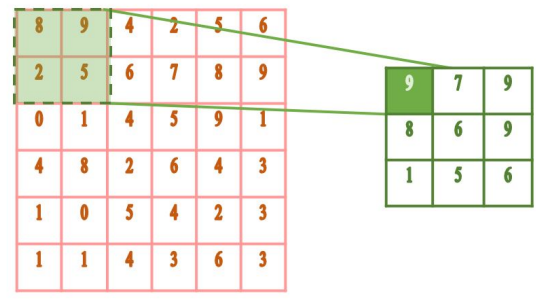

B.

Figure 2.4: Two main layers of CNNs. A. Convolutional layer with a filter size of $3 \times 3$ and stride of 1 . B. Max-pooling layer with filter size of $2 \times 2$ and a stride of 2

\subsubsection{Convolutional layers}

The robust ability in representation learning for CNNs is concentrated on convolutional layers, where it comprises a set of learnable localized and translational filters. Each filter performs a convolution operation across the input spatial domain, producing an output feature map that represents the filter's responses at every spatial position of the input (see Fig. 2.4A.). Such filters learn multiple features in parallel for a given input and produce a single response to each filter as an output feature map, which represent several localized features. Similar to standard NNs, the forward and back-propagation algorithms are used to train the CNN and estimate parameters. A gradient-based optimization method is utilized to minimize the loss function and update each parameter of the filter weights. Stacking several building blocks, CNNs allow models to hierarchically learn features at multiple levels of abstraction, meaning complicated concepts can be learned from simpler ones and more generalized versions of a feature detector of the input image. Filters in earlier layers of a network learn low-level features (e.g. edges, curves and corners), while filters in later layers learn more complex concepts (e.g. parts and objects) [70]. This hierarchical structure is prevalent in image-processing, which justifies why CNNs work well for image recognition without the need to apply pre-processing techniques such as hand-crafted features used in traditional image processing methods.

\subsubsection{Feature Map, Padding, and Stride}

A feature map is the output of a convolutional layer. When feeding data samples through the network, each is represented differently and has individual feature maps throughout all filters. In CNNs, the feature map of each filter refers to all calculations from all of the previous layers that may affect the obtained output during the forward passing. Feature maps can be observed 
as the learned representations or features in the spatial dimensions. The size of feature maps is determined by the input's size and the convolution filter's size and can be controlled by three parameters: depth, stride, and padding. Depth represents the number of filters used for the convolution operation, while stride is the number of shifted pixels based on which the filter is slid over the input image. When the stride is 2 , the amount of movement is 2 pixels at a time. A larger value for stride can produce much smaller feature maps. Padding adds additional filler pixels around the input image boundary to avoid losing pixels on the perimeter. Stride, padding, and kernel size techniques can be incorporated to control the overall size (e.g. width and height) of feature maps.

\subsubsection{Pooling layers}

A pooling layer usually follows a sequence of one or more convolutional layers as a way to consolidate the feature representations learned in the previous layers. As pooling layers have no trainable weights, they perform a down-sample operation to the input feature maps through striding an aggregation function (e.g. maximum) across each receptive field in the input feature maps. As a result, the values of the covered receptive field are reduced to a single value in the produced feature map (see Fig. 2.4B.). Pooling layers generally apply a simple statistic operation, taking the maximum or the average of the covered receptive fields in order to produce its own feature map [71]. The main goal of the pooling layers is to reduce memory usage, computational load, and the number of parameters [68], where the spatial size of the data feature maps generated by successive convolutional layers is progressively reduced, consequently reducing the overall size. As with convolutional layers, stride, padding, and kernel size can modify the operation to achieve the desired output size.

\subsubsection{Convolutional Neural Networks (LeNet)}

LeCun et al.'s 1998 paper [68] was a significant breakthrough; in it, the famous CNN LeNet-5 was introduced to recognize handwritten zip code numbers. LeNet-5 is one of the first neural networks to be successfully introduced during the developing phase of deep learning because it can be better appropriate in image-processing tasks where it exploits the local information through convolutional layers. Its architecture is straightforward and is composed of five layers, three of which are convolutional layers and two of which are fully-connected (see Fig. 2.6A.). Since then, the architectures of typical CNNs have consisted of several convolutional stages, where each stage has a convolutional layer generally followed by an activation layer, and each 


\section{Background}

stage ends with a pooling layer. Progressing through the network, the input image becomes smaller and more abstract. At the final stage, several fully-connected layers are added, and the final layer outputs the prediction.

\subsubsection{Modern Convolutional Neural Networks}

Although LeNet achieved positive results in handwritten recognition problems, the performance and feasibility of training CNNs on larger, more realistic datasets has yet to be established [6]. Important developments in the field of CNNs have expanded and evolved CNN architectures to build deep networks and learn more complex functions. In 2012, AlexNet [9] won the ImageNet Large Scale Visual Recognition Challenge, achieving state-of-the-art performance on the ImageNet database [72] at its time. AlexNet is a classic CNN architecture and is considered the first deep, large-scale network, encouraging the use of CNNs in real-world applications. It consists of eight weighted layers, five of which are convolutional, followed by three fully-connected layers, of which the third layer is the output layer (see Fig. 2.6B.). ReLU was utilized as an activation function in the AlexNet rather than the sigmoid function. AlexNet provided experimental proof that deep CNNs can achieve desirable results, but limited attention has been paid to providing general guidance for designing a worthwhile CNN model.

The idea of using blocks when designing CNNs was first introduced by the Visual Geometry Group (VGG) at Oxford University. Each building block of their CNN architecture consists of convolutional layers, ReLU activation functions, and a max-pooling layer. VGGNet [10] is a CNN architecture for large-scale image recognition, designed for the ImageNet dataset. VGG16, for instance, consists of sixteen layers, of which the first thirteen are convolutional with a filter size of $3 \times 3$ with a stride of 1 and a pooling region of $2 \times 2$ without overlap, reducing the size of feature maps after each block (see Fig. 2.6C.). The success of VGG lies in the adoption of a small kernel-sized filter (i.e. $3 \times 3$ ) to allow the network to extract more complex features, overcoming the issues of applying the large kernels used in AlexNet. The use of smaller kernels can reduce the number of training parameters and increase the depth of the network, allowing the learning of high-level, non-linear representations.

The achievement of CNNs raised the necessity to design networks that are more powerful and expressive rather than merely deep. Examining the design of CNN architectures evolved from LeNet, AlexNet, and VGGNet, their focus was on designing deeper networks in order to widen learning capacity, extract more complex features, and learn features at multiple levels of abstraction. Although networks that are too deep are more likely to overfit the training 
data [11], the use of regularization techniques (i.e. dropout layers [56], and batch normalization [55]) has proven very effective in improving the generalization and avoiding overfitting $[57,58]$. However, the issue of vanishing gradients remained an obstacle that inhibits the development of deep neural networks.

GoogLeNet [42], also known as the Inception network, was introduced by researchers at Google and won the ImageNet Challenge in 2014. Said researchers proposed a deeper network with far fewer parameters, 12 times fewer than VGGNet, to improve computing resource utilization. The novel element of this architecture is the introduction of inception blocks. Unlike classical architectures, where layers are stacked on top of each other, an inception block consists of several convolutional layers in four parallel paths with various kernel sizes (i.e. $1 \times 1$, $3 \times 3$, and $5 \times 5$ ), followed by concatenation to comprise the blocks output (see Fig. 2.5A.). All four paths use suitable padding to satisfy the dimension of input and output feature maps. The GoogleNet architecture consists of 22 layers, nine of which are inception blocks. A $3 \times 3$ pooling layer is added after every several inception blocks for downsampling, and a global average pooling is added at the end to avoid the use of fully-connected layers (see Fig. 2.6D.). The GoogLeNet has shown to be effective because it explores the image in a variety of filter sizes, allowing to efficiently recognize details at different extents [6].

The Residual Neural Network (ResNet) [11] was introduced as a novel architecture with skip connections known as residual connections, which allow the training of very deep neural networks with 50,101, and 152 layers and solve the vanishing gradient problem. ResNet achieved state-of-the-art performance for the ImageNet database and outperformed all prior CNNs. The residual connections are applied to pass information across layers, creating an alternative shortcut path for the gradient to pass through the residual connections (see Fig. 2.6E.). Learning an identity function is another advantage of residual connections. ResNets have several stages of residual blocks, each of which has two $3 \times 3$ convolutional layers with an equal number of output channels, followed by a batch normalization layer and a ReLU activation function. The two convolution operations are skipped by connecting the original input directly to the output of the second convolution block before the final ReLU activation of the residual block. Identity shortcuts are directly used when the input and output comprise the same dimensions. When the feature maps are down-sampled, the shortcut performs by $1 \times 1$ kernels (see Fig. 2.5B.). This procedure overcomes the issue which occurs when the shortcuts go across feature maps of two different sizes.

The development of several robust CNNs approaches and their utilization in major prob- 


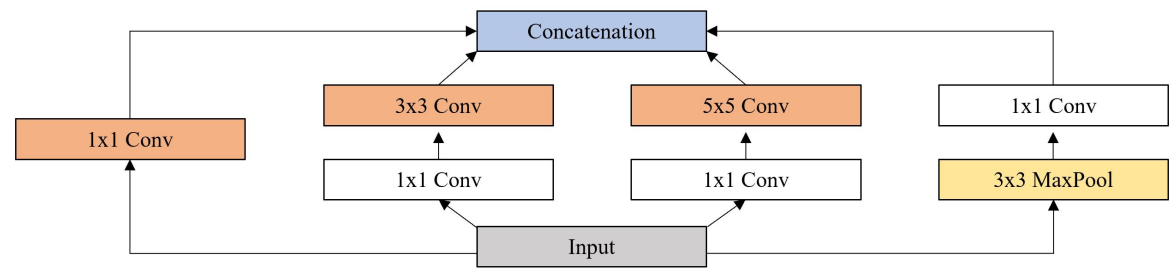

A. Inception block.
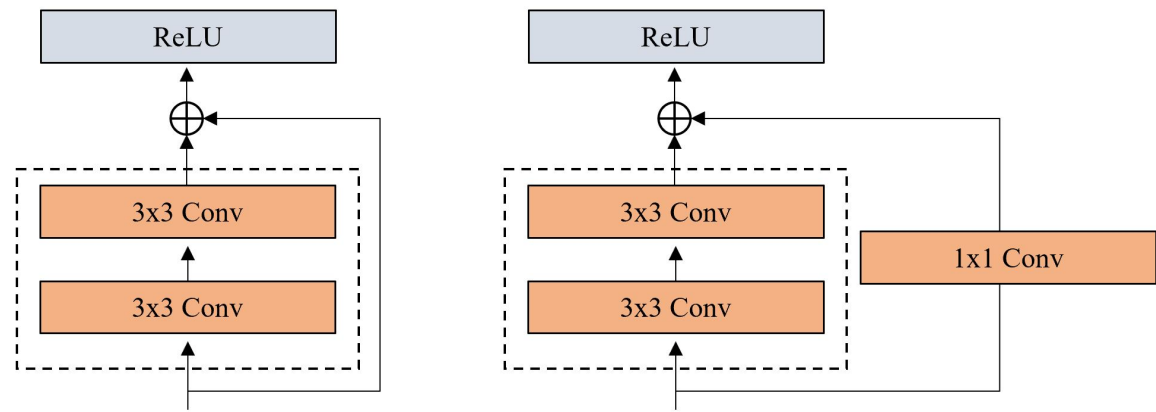

B. ResNet block

Figure 2.5: Structure of the Inception and ResNet blocks.

lems made them the center of attention. Convolutional neural networks (CNNs) can be seen as powerful tools that compose the backbone of most advanced computer vision systems [6]. We have introduced popular deep models which are related to our research and commonly used in the vision community. However, CNNs have also attained extraordinary achievement in other recognition tasks (i.e., object detection [7,73-77] and semantic segmentation $[8,78,79]$ ), thereby becoming an indispensable method used for a wide variety of applications.

Object detection identifies specific positions of one or multiple objects in an image. The use of this task extends across many fields; such as self-driving technology, robots, and systems in the security field to detect targets of interest or identify their locations. Regions with CNN features (R-CNNs) [73] is considered a pioneering work that introduced deep models to object detection. In the R-CNN model, several proposed regions from an image are selected by the selective search algorithm [80], and a CNN is then utilized as a feature extractor to learn features from each proposed region. These features are then fed to a classifier, which predicts offset values for bounding boxes of the proposed regions and their categories. Following this work, a series of improvements to the R-CNNs have been developed. Fast R-CNN [7] performs $\mathrm{CNN}$ forward computation using the whole image. The learned feature map allows for 


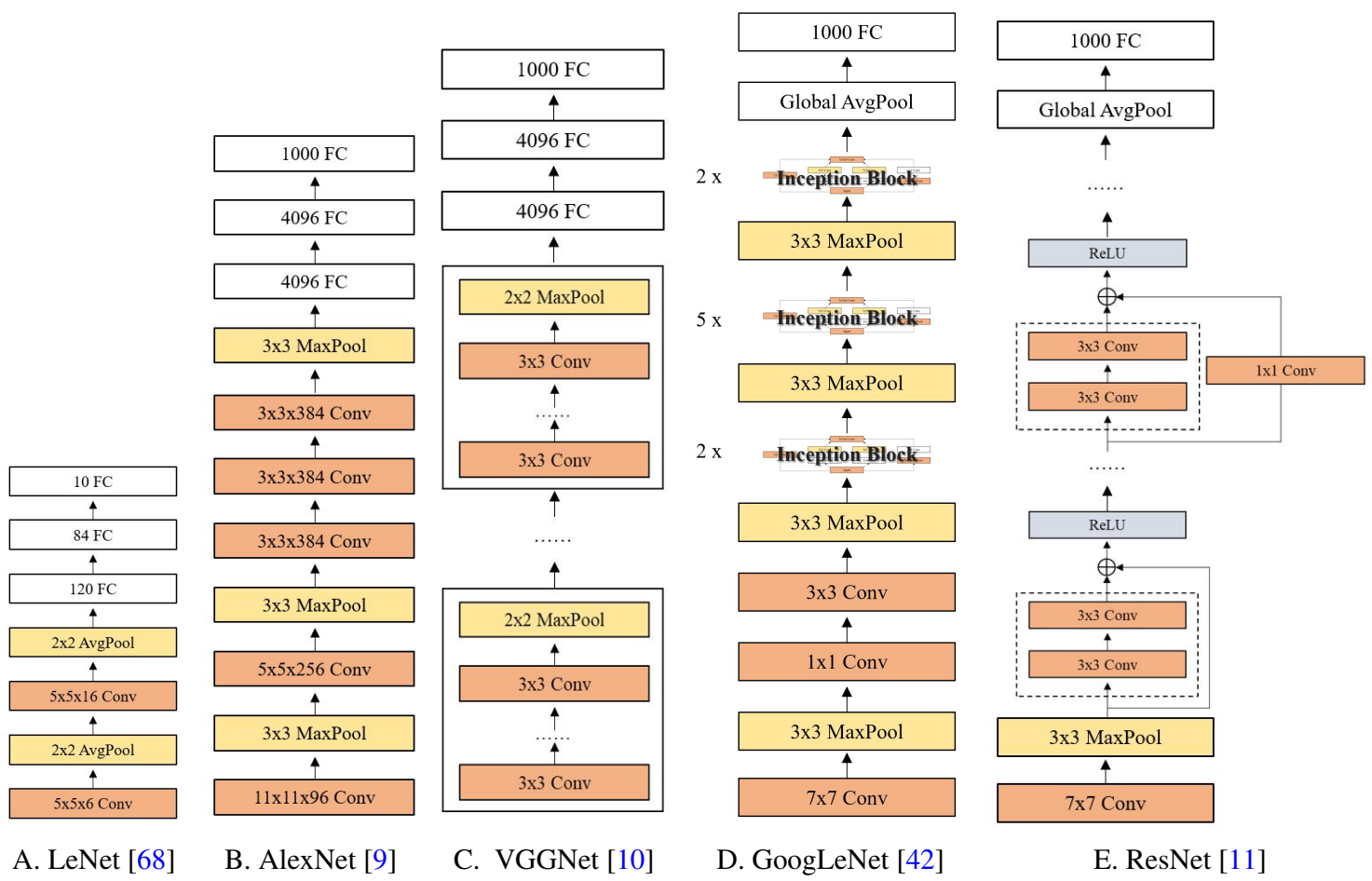

Figure 2.6: The development of CNN Architectures.

the identification of the proposed regions. A Region of Interest (RoI) pooling layer is introduced to extract features of the same shape from RoIs of different shapes. As a result, the extracted features are reshaped into a fixed size and used as inputs to fully connected layers, in order to predict the category and offset values for the proposed region's bounding box. Faster R-CNN [74] improved the Fast R-CNN by replacing the selective search on the feature map with a region proposal network, reducing the number of proposed regions. The RoI pooling layer is then utilized to reshape the predicted regions used to predict an object's final detection. Later, Mask R-CNN [77] was developed on top of Faster R-CNN. Here, the RoI pooling layer is replaced with an RoI alignment layer, which employs bilinear interpolation to preserve spatial information on feature maps. Along with predicting the bounding boxes and categories of RoIs, the RoI alignment layer allows for the addition of a fully convolutional layer to localize objects at the pixel level.

Semantic segmentation attempts to segment images into regions with different semantic categories, predicting objects or regions label at the pixel level. Papandreou et al. [81] used a CNN model for semantic image segmentation training under weakly supervised and semi- 


\section{Background}

supervised settings using image-level labels. A fully convolutional network (FCN) [78] is another deep semantic segmentation model that utilizes CNNs to map image pixels into pixel categories. In contrast to CNNs, an FCN first applies convolutional layers to extract image features, which are then transformed into several categories using the $1 \times 1$ convolutional layer. Each pixel's output category is learned by transforming the feature maps to the same size as that of the input image utilizing the deconvolution layer. The Pyramid Scene Parsing Network (PSPNet) [79] is also a framework for semantic segmentation. The PSPNet model firstly extracts the feature map by adopting ResNet [11]. The learned features are then fed to a pyramid pooling module to recognize patterns with different scales. They are pooled with four different scales, each corresponding to a pyramid level, and processed by a $1 \times 1$ convolutional layer to reduce their dimensions. The pyramid levels' outputs are upsampled to a standard scale and concatenated together, processed later by a convolutional layer to generate the pixel-wise predictions.

\subsubsection{Auto-encoders}

An auto-encoder (AE) is a deep unsupervised model for representation learning, consisting of encoding and decoding parts. AEs are a specific type of neural network capable of learning efficient representations of the input data without supervision. The data $X$ is projected into a set of feature spaces $H$ using the encoding part, from which the decoding part reconstructs the original data $\hat{X}$ (see Fig. 2.7). The training is performed in an unsupervised manner by minimizing the differences between the original and reconstructed data with distance metrics. Like standard NNs, the weights of the AE are optimized using an optimizer and back-propagation. The most common loss function of an AE is Mean Squared Error (MSE) [82], given by the following form:

$$
E=\frac{1}{N} \sum_{i=1}^{n}\left\|x_{i}-\hat{x}_{i}\right\|^{2},
$$

where $\hat{x}$ is a reconstructed image, and $x$ is an original image.

In this procedure, the input is mapped into new space representations, allowing useful features to be obtained through encoding procedures. The AE point of interest is the encoding (middle) part, in contrast to standard NN architectures which use the final output as the point of interest. The encoding part makes AEs robust feature detectors and useful for dimensionality reduction. Deep auto-encoders (DAEs) and deep convolutional auto-encoders (DCAEs) are the most common types of AE. The significant difference between DAE and DCAE is that the 


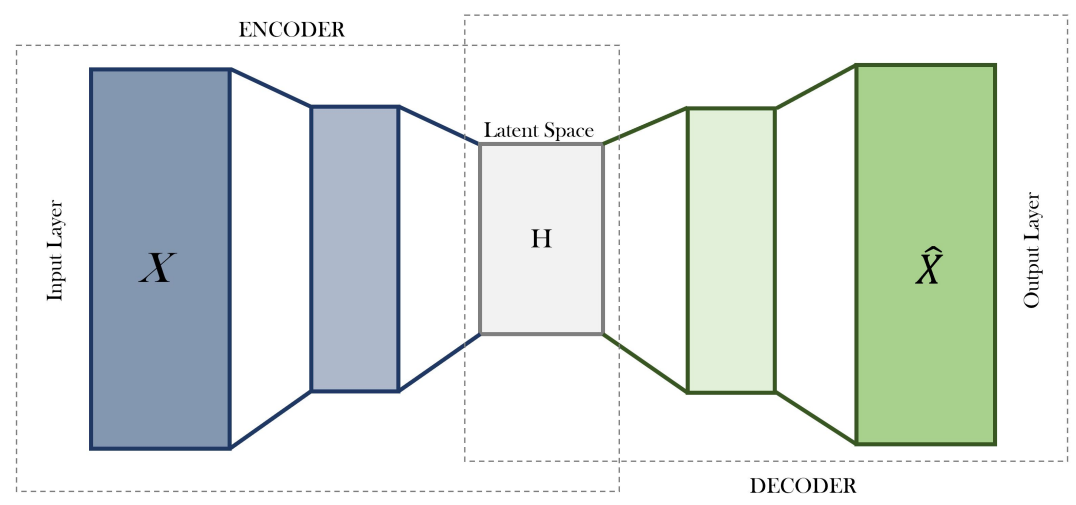

Figure 2.7: An Auto-encoder (AE) framework, where the input $X$ is projected into a lower-dimensional space $H$ and then reconstructed to an output representation $\hat{X}$. The hidden layers will have weights optimized such that the output representation is as similar to the original data as possible.

former adopts fully-connected layers to globally reconstruct a signal, while the latter utilizes local information to achieve the same objective. Moreover, DCAEs may be better suited for image processing as they fully utilize the properties of CNNs, which are shown to outperform all other techniques used on image data [83,84]. These properties, mainly local connection and parameter sharing, make CNN suitable to have a property in translation latent features.

In contrast to the DAE model, which uses matrix multiplication to perform full connectivity on the node connection, DCAE [85] uses a convolution operation to force spatial connectivity through convolutional and deconvolutional layers. Deconvolutional layers were proposed in conjunction with unpooling (or upsampling) layers [86] to map the activations of the encoding layer back to pixel space. In the encoding parts, convolutional layers are used as feature extractors to learn features by mapping the data into an internal layer. A latent representation of the $n^{\text {th }}$ feature map of the existing layer is given by the following form:

$$
h^{n}=\sigma\left(x * W^{n}+b^{n}\right),
$$

where $W$ is the filters and $b$ is the corresponding bias of the $n^{\text {th }}$ feature map, $\sigma$ is the activation function (e.g. sigmoid, ReLU), and $*$ denotes the $2 \mathrm{D}$ convolution operation.

In contrast, the deconvolutional layers invert this process and reconstruct the latent representation back into its original shape, thus, this process maps the obtained features into pixels [87] by using the following form:

$$
y=\sigma\left(\sum_{n \in H} h^{n} * \tilde{W}^{n}+c\right),
$$




\section{Background}

where $H$ denotes the group of latent feature maps, $\tilde{W}$ is the flip operation over both dimensions of the weights, $c$ is the corresponding bias, $\sigma$ is the activation function, and $*$ denotes the 2D convolution operation. The difference between convolution operations in Eq.(2.12) and Eq.(2.13) is that the convolutional layer performs a valid convolution which decreases the output size of feature maps, while the deconvolution layer performs a full convolution which increases the output size of feature maps [85,88]. In other words, if $x$ is an $m \times m$ image and the filters are $n \times n$, then the valid convolution performs $(m-n+1) \times(m-n+1)$ and full convolution performs $(m+n-1) \times(m+n-1)$.

\subsubsection{Advances in Deep Learning}

Other deep unsupervised models for representation learning that leverage CNN's power of localized feature learning have been developed. Goodfellow et al. [89] introduced Generative Adversarial Networks (GANs), a breakthrough concept which makes use of the discriminative models to produce good generative models. GANs rely on the adversarial interactions between generative and discriminative models [90-92]. A generative model produces samples from latent variables, while the discriminative network attempts to distinguish between samples produced from an actual data distribution and those produced by the generator. The discriminator and generator are optimized in an attempt to maximize the discriminator's ability to get better at distinguishing real and fake samples, and the generator's ability to generate better samples to trick the discriminator.

Other advanced deep learning architectures have been developed in the context of timeseries analysis, including prediction, which aims to deduce from data collected in the past and reveal how the data will develop in the future. Recurrent Neural Networks (RNNs) [12,93] and Long Short-Term Memory (LSTM) [54] are the most commonly used techniques for this task. These methods produce a feedback loop by using a layer's outputs as inputs to the same layer and make use of internal memory to remember information about previous steps. In their early development, the RNNs suffered from the exploding and vanishing gradient problem, as they rely on the backpropagation through time when calculating the gradient. In order to overcome the gradient challenges of standard RNN and achieve more effective learning, the gated memory units of an LSTM are utilized as building units for RNN's layers, where the backpropagated error is robust to degradation. LSTMs introduce a kind of storage within a network over time, enabling RNNs to remember inputs over a long time. Such networks have been increasingly used with sequential data, including speech recognition and translation 
analysis $[93,94]$, resutling in state-of-the-art performance.

\subsubsection{Visualizing, Explaining and Interpreting Deep Learning}

Deep learning algorithms are often perceived as black-box models due to their ambiguity and unclear working mechanisms [12]. Visualization techniques are developed to explore such complex models as well as illustrate and explain their internal operation and work mechanisms, as there is no clear understanding of why deep classification algorithms achieve highly performant results. These techniques allow the community to gain general insights and obtain an overview of how to control and improve such models. Efforts have been made in the field of computer vision to clarify the learned features of deep learning models and provide a clear understanding of the internal operation and work mechanisms of deep networks. Several approaches have been developed to visually understand convolutional layers (i.e. code inversion $[70,95,96])$ and activation maximization [97-99]), interpret deep visual representations and quantify their interpretability $[100,101]$, as well as measure the influence of hidden units on the final prediction [102-104]. Furthermore, a set of visualizations have been developed to help machine learning experts clearly understand such deep complex models (e.g. [105, 106]). Liu et al. [105] have recently presented an interactive visual analytics approach which allows for the better understanding, diagnosis, and improvement of deep CNNs.

\subsubsection{Visualizing and Understanding Deep Learning Models}

Several approaches have been introduced to understand the learned feature obtained via CNNs. Zeile et al. [70] developed a novel visualization technique that makes use of deconvolution and unpooling layers to study and analyze the intermediate representations, allowing for the inspection of the evolution of features during training and diagnosis of possible problems with the model. The DeconvNet architecture aims to approximately reconstruct each layer's input from its output, mapping the activations of intermediate layers back to a pixel space, which describes a filter's response to a particular input image. They show that filters in earlier layers of a network learn low-level features, while filters of later layers learn more complex concepts for a given class. Mahendran et al. [96] adopted the same approach. They developed a method to analyze the visual information in learned representations by computing an approximate inverse of an image representation. In another approach, Erhan et al. [97] visualized the hidden layers' features by maximizing the activation to find the optimal stimulus for each unit. Their aim was to find qualitative explanations of high-level feature representation by simply weighing 


\section{Background}

the filters at the previous layer using the connections with the largest weights. The main focus of these methods is to understand a model's predictions by analyzing the individual units and seeking an explanation for specific activation.

\subsubsection{Explaining and Interpreting Deep Learning Models}

The interpretation of deep learning models is accompanied by multiple challenges due to their size, complexity, and often ambiguous internal state; this has lead to an increasing development of practical and robust methods to explain deep networks' decisions [107-109]. In particular, Layer-wise Relevance Propagation (LRP) [104] is one of the most important methods in explaining deep network decisions [110]. It computes a measure of relevance quantity and determines important pixels with high relevance $R$ in the input through a backward procedure. LRP calculates each neuron's summed relevance quantity in the network to the overall classification score, decomposing a classification decision into contributions for each neuron. This procedure allows LRP to explain individual network decisions and identify the important regions in the image for the classification decision. The principal characteristic of this method is that during the backward pass, the network output is redistributed to all elements of the network in a layer-by-layer fashion: at each layer, the unit that contributes the most to the next layer will receive the most relevance from it, conserving fair relevance redistribution. Let $R_{i}^{(l)}$ represent the relevance of a neuron $i$ in $l$ layer and $R_{j}^{(l+1)}$ denotes the relevance of a neuron $j$ of the next layer $l+1$, this is defined as:

$$
R_{i}^{(l)}=\sum_{j} R_{j}^{(l+1)} .
$$

To satisfy this equation, Bach et al. [104] presented the relevance propagation rule, defined as:

$$
R_{i}^{(l)}=\sum_{j} \frac{z_{i j}}{\sum_{i} z_{i j}+\varepsilon} R_{j}^{(l+1)}
$$

where $z_{i j}$ is the relevance quantity that presents a value to which neuron $i$ has contributed to make neuron $j$ relevant, according to their contributions to the neurons in the next layer $l+1$. The constant $\varepsilon$ adds a small positive term to prevent the denominator from becoming zero. Other propagation rules have been proposed [110] to suit other layers (i.e. pooling [111], and LSTM blocks [112]).

Further methods interpret deep visual representations and quantify their interpretability $[101,113,114]$, explaining deep network decisions in terms of the learned features in latent 
space. In particular, [115] was introduced to estimate how important a concept is, providing an internal-space explanation for every particular prediction. They used directional derivatives to measure the degree to which a concept is essential to a classification result. Network Dissection [100] was also developed to quantify the interpretability of latent representations of CNNs that reflect the contribution of an input to deliver essential information for the final prediction of the model. To measure channels' importance, the latent representations of every individual feature map are evaluated as a solution to a binary segmentation task of the visual concept in the input space. Determining the function of individual filters in a CNN and their ability to localize the meaningful semantic part both aid to efficiently measure the importance of different feature maps. After the activation matrix is calculated, the distributions of individual feature map $j$ are computed, based on which a top quantile value is determined over every spatial location of the feature map. The top quantile value is used as a threshold $T$ to produce a binary matrix for each channel in the latent space. Here, the output feature map $t_{j}^{(i)} x_{n}$ is thresholded into a binary segmentation $M$, where all regions that exceed the threshold are selected. If a channel in hidden layers has feature maps that are smaller than the input resolution, they are scaled up to match the input resolution using bilinear interpolation. The interpolating function assigns each missing pixel by taking the weighted average of the nearest pixels. The importance of every individual channel $M_{j}\left(t_{j}^{(i)} x_{n}\right)$ is evaluated by computing intersection over union score between their binary segmented versions against the input binary segmentation $I\left(x_{n}\right)$.

$$
I o U_{j}=\frac{\left|M_{j}\left(t_{j}^{(i)} x_{n}>T\right) \cap I\left(x_{n}\right)\right|}{\left|M_{j}\left(t_{j}^{(i)} x_{n}>T\right) \cup I\left(x_{n}\right)\right|} .
$$

Some methods combine multiple approaches (e.g., different layers of the neural networks) to achieve a more informative explanation of the prediction process $[116,117]$. Other recent works on interpreting GANs have been proposed [101,118], where structured textual explanations of deep learning models have been built. Bau et al. [101] has proposed to visualize and understand GANs by identifying interpretable latent representations that match some object concepts. They demonstrated that a subset of internal units essentially contributes to the particular objects generating for GAN's outputs.

Finally, the quantitative assessment of neurons' property has been adopted to understand neuron property and evaluate neurons' importance. Such techniques introduce objectives to measure the activation values of each neuron and assign to them a score. Dhamdhere et al. [102] utilized integrated gradients by summing the gradients of the output prediction with respect to 


\section{Background}

the input, in order to evaluate the importance of hidden neurons. Amjad et al. [119] also proposed a method to compute internal neuron importance, utilizing information-theoretic quantities (i.e. entropy and mutual information) to understand the outputs of individual neurons of trained neural networks. Moreover, Morcos et al. [45] investigated the relationship between the classification performance of neural networks and the output of individual neurons to estimate class selectivity and mutual information for the activation of each neuron. In addition to this, $\mathrm{Na}$ et al. [103] have recently used the highest mean activation to measure the importance of individual units on language tasks, showing that different units are selectively responsive to specific morphemes, words and phrases.

\subsection{Clustering}

\subsubsection{Conventional Clustering Methods}

Clustering is a machine learning technique that is widely used as an unsupervised method. A clustering algorithm aims to define a grouped structure of similar objects in unlabeled data based on their similar features. Consequently, data in one cluster is homogeneous with each other and dissimilar to the data in other clusters. Features do not provide any information about an appropriate group for its objects; they only describe each object in the dataset, assisting clustering algorithms to learn and extract useful information for their structure. We provide a review of popular clustering algorithms, which can be divided into four methods: partitioning methods, hierarchical methods, model-based methods, and density-based methods.

\subsubsection{Partitioning Methods}

Partitioning methods are described as the process of partitioning unlabeled data into $K$ groups. Kmeans, Kmedoids (PAM), Fuzzy Cmeans, and Fuzzy Cmedoids are the most popular algorithms for partitioning clustering. Kaufman et al. [120] categorized these algorithms into two categories: crisp (hard) clustering methods (including Kmeans and Kmedoids) and fuzzy (soft) clustering methods (including Fuzzy Cmeans and Fuzzy Cmedoids). While in hard clustering methods, each object is assigned to only one cluster, in fuzzy clustering methods, each object is assigned to more than one cluster with a probability. In such methods, the number of clusters must be pre-assigned and most partitioning algorithms cannot tackle the problem of finding the number of clusters [120]. 
Kmeans [121] is a simple and widely used algorithm which divides a set of data into K groups represented by their mean values. After K cluster centers (centroids) are randomly initialized, each example is assigned to the nearest cluster center. It iterates until it converges to a locally optimal partition of the data. For each iteration, each example is assigned to the closest cluster center, which will be recalculated based on the mean value of all examples of that particular cluster [122]. The use of the Kmeans algorithm was preferred due to its speed, simplicity, ease of implementation, and the possibility of assigning the desired amount of clusters [123, 124]. Kmedoids or PAM (Partition Around Medoids) [120] is another partitioning algorithm in which a set of K representative samples are initially selected, before each example in the dataset is assigned to the nearest representative sample, constructing partitioned clusters. Although this algorithm is like the Kmeans algorithm, it can be more robust to noise and outliers because it minimizes a sum of general pairwise dissimilarities and only differs in its representation. Instead of implying a mean, Kmedoids clusters are represented by the representative data sample in each cluster.

Fuzzy clustering algorithms aim to minimize an objective function that usually has numerous undesirable local minima [125], allowing fuzzy partitioning instead of hard partitioning. Thus, each sample in the dataset could be assigned to more than one cluster with a membership that measures degrees of association to clusters. Although fuzzy clustering algorithms are usually more time-consuming, they provide more detailed information concerning the data structure [120]. Fuzzy Cmeans [126,127] is the most common fuzzy clustering algorithm and an extended version of Kmeans. It provides both effective and significantly meaningful (fuzzy) data partition [128]; moreover, this algorithm was later improved [128-131]. Here, a dataset is divided into fuzzy groups that differentiate in representatives by minimizing the objective function (within groups) of weighted coefficients (e.g. distances between objects and cluster center), influencing the fuzziness of membership values. Fuzzy Cmedoids [132] is another fuzzy partition algorithm which is an extended version of Kmedoids. The candidate medoids are picked (as objective functions located in the cluster center) from the dataset to minimize all fuzzy dissimilar objects in the cluster.

\subsubsection{Hierarchical Methods}

Hierarchical clustering defines a tree structure for unlabeled data by aggregating data samples into a tree of clusters. This method does not assume a value of $\mathrm{K}$, unlike Kmeans clustering. There are two main kinds of hierarchical clustering methods - agglomerative (bottom-up) and 


\section{Background}

divisive (top-down) [133, 134].

An agglomerative algorithm (bottom-up) considers each object as a cluster and then progressively integrates clusters. The merging process is repeated until all items are in one cluster or termination conditions are satisfied, such as the number of clusters being sufficient. The divisive algorithm (top-down) starts by grouping all objects into one cluster, then divides the cluster until each object is in a separate cluster [133, 134]. Bernard et al. [135] described two advantages of divisive clustering for data. Firstly, the hierarchical structure allows for multiple levels of detail with the same data elements in respective sub-trees. Secondly, the level of detail concept can be achieved with a single calculation. However, both algorithms predominantly suffer from an inability to perform adjustments once a combining or dividing decision has been implemented, and they lack the ability to undo what has previously been done $[120,122,136,137]$.

The basic hierarchical clustering algorithm starts by assigning each vector to its own cluster, then computing the distances between all clusters and saving these distances into a distance matrix. Next, it finds, through the distance matrix, the two closest clusters or objects which will produce a cluster. It updates the distance matrix and returns to the previous step until only one cluster remains [136]. Hierarchical algorithms usually employ a similarity or distance matrix to merge or split one cluster, and this can be visualized as a dendrogram [122]. Lin et al. [138] present Symbolic Aggregate Approximation (SAX) representation and use hierarchical clustering to evaluate their work. Hierarchical clustering methods can also be divided based on the way that the similarity measure is calculated; examples include single-link clustering, average-link clustering, and complete-link clustering [122]. CURE [139], BIRCH [140], and Chameleon [141] are some examples for improving the performance of hierarchical clustering algorithms. Hierarchical methods can produce multi-nested partitions that let different users select diverse partitions based on the similarity level required, but suffer from computational complexity in time and space, and clustering many objects incurs a substantial I/O cost.

\subsubsection{Model Based Methods}

Gaussian mixture modelling (GMM) $[142,143]$ is a model-based approach to data clustering which is described as a weighted sum of Gaussian functions. GMM involves a mixture of multiple Gaussian functions, all of which allow for the learning of the distribution of data in a given space. It is also considered a centroid-based clustering algorithm, which divides a set of d-dimensional data into K groups represented by several parameters. As all data samples are 
assumed to be generated from a mixture of a number of Gaussian distributions with unknown parameters, learning Gaussian distributions of such data can provide much better clustering results than Kmeans, where a single radii attains limited results to constrain the cluster boundaries. The parameters of GMM give an insight into the clustering confidence. Although GMM can be considered an extension of Kmeans, it differs in a number of ways. GMM performs soft clustering, whereas Kmeans performs hard clustering. Moreover, instead of describing each cluster by only its centroid, GMM represents a cluster by its centroid (mean), standard deviation or covariance matrix for each Gaussian function, which can be conveniently computed from clustered feature representations and the size of the cluster (weight), which is itself computed by counting the amount of data belonging to each Gaussian component. GMM is a probabilistic model because it can give probabilities of each point being in a specific cluster, which are used to predict the clustering output.

A self-organizing map (SOM), a model-based method developed by Kohonen [144], is a specific type of neural network $(\mathrm{NN})$ used for model-based clustering. As an unsupervised learning method, self-organizing neural networks rely on neurons coordinated in a lowdimensional (often two-dimensional) structure. Those neurons are iteratively trained by the self-organizing procedure during which the topological ordering of the weight vectors takes place. SOM is one of the most common neural network models and is often used for data analysis. It is also described by Kohonen as an analysis and visualization tool for high-dimensional data [145]. However, SOM can also be used for other applications such as clustering, sampling, dimensionality reduction, vector quantization, and data mining [146,147]. The most important feature of SOM is produced in the output layer by the neighborhood relationship [148].

Various extensions have been developed to enhance the SOM's scope and performance, such as adaptive subspace SOM (ASSOM) [149, 150], the parameterized SOM (PSOM) [151], visualization induced SOM (ViSOM) [152,153], and the Self-Organizing Mixture Network (SOMN) [154]. A SOM uses a collection of neurons usually arranged in a 2-D hexagonal or rectangular grid to shape a discrete topological mapping of input space. At the beginning of the training process, weights are initialized by assigning small random numbers. In this algorithm, each training iteration has three stages. First, an input is presented every time, and then the best matching cell, or winning neuron, is selected. After that, the weight of the winner and its neighbors are updated. The process is repeated until the map converges and the weights have stabilized. In the feature space, the neighboring locations are always represented in the neighboring neurons in the network because they are updated at every step. During the 


\section{Background}

mapping, the topology of the data is maintained as it was in the input space [155-157].

\subsubsection{Density-Based Methods}

In density-based clustering, the cluster continues to expand if the density of a set of points with its neighbors is closely packed together, and that cluster is separated by subspaces where the objects have low density. This kind of algorithm is more complex than other clustering algorithms such as partitioning clustering [134]. As it is based on data density, density-based clustering can distinguish noise data and does not require a prior number of clusters, which can be more helpful for non-linear clustering. DBSCAN [158], OPTICS [159] and LOF [160] are some of the common algorithms that work with the density-based concept.

DBSCAN (Density-Based Spatial Clustering of Applications with Noise) [158] is one of the most highly cited density-based methods. It depends on a density-based concept of clusters which is designed to detect clusters and noise in a set of data. For each point of a cluster, the eps-neighborhood (eps) must have a minimum number of points (minPts). Therefore, the two parameters, eps and minPts, must be known for each cluster or, at the very least, for one point from the particular cluster. Every cluster contains two sets of points, the core and border points, which are on the cluster's border. DBSCAN is efficient for large datasets and aims to discover clusters of arbitrary shapes, but cannot transact with clusters of various densities, which is one of the algorithm's main problems. In contrast, OPTICS (Ordering Points To Identify the Clustering Structure) [159] can deal with the issue of an unknown number of clusters with different densities [161]. Local Outlier Factor (LOF) [160] also shares certain notions with DBSCAN and OPTICS with regard to local density estimation, and depends on distances in its local neighborhood. Most clustering algorithms are developed to find and optimize clustering, and usually ignore noise when the clustering result is produced, however, the LOF tries to assign for each object a degree of being an outlier.

\subsubsection{Deep Clustering Methods}

Deep learning [21,32] has forged a transformational path in machine learning algorithms , finding most fame in supervised learning. Many supervised models depend on a preliminary unsupervised learning step, known as unsupervised pertaining, where the right representations for data are learned and exploited to improve training and the performance of supervised models. The unprecedented achievement of deep learning has inspired researchers to develop deep learning-based methods for clustering analysis. High-level representations provide beneficial 
components that support traditional algorithms to demonstrate satisfying performance and allow them to deal with latent abstract representations in a given space. Deep learning has been exploited for the purpose of clustering, where its representational power provides latent representations as inputs for clustering algorithms so clusters are easier to extract and clustering quality is improved. Although some approaches apply clustering as an enabling step to enhance their representations, most of the focus was on providing different procedures to obtain richer deep representations, with some reference to deep clustering. However, deep representations and clustering outputs are the results of such approaches; we therefore use the Deep Clustering term to refer to all the methods introduced in this section.

Different approaches to deep clustering methods have been developed utilizing the power of deep neural networks. The deep auto-encoder (DAE) and deep convolutional auto-encoder (DCAE) have been shown to be efficient approaches that can be used to extract features and reduce dimensionality in an unsupervised manner. These deep approaches are commonly used models for deep clustering. In this section, we mainly focus on deep clustering methods, where feature representations learned through deep networks are utilized for the purpose of clustering. Deep clustering methods can be different in several key respects, including network architectures, algorithmic structure, loss functions, and optimization.Existing works can be classified into three categories based on their design and the overall procedural structure. In this classification, we consider how the clustering algorithm is applied. First, some methods consist of two main steps, where they extract latent features for given data and then perform clustering on the learned representations. In another category, some methods use an iterative loop to improve the procedure of the first category, although it still comprises the two salient steps. In the last category, researchers have embedded a clustering algorithm into deep neural networks, where feature representations and clustering assignments are simultaneously learned, applying joint loss function.

All three classifications of deep clustering are based on core components (i.e. representation learning and clustering loss functions) that are fundamental in designing deep clustering methods. A common factor between most of the clustering methods is that they deal with deep learned features rather than raw data. Traditional clustering algorithms demonstrate limited performance as dimensionality increases, but deep neural networks minimize this issue by allowing a clustering algorithm to deal with a clustering-friendly representation instead of high-dimensional data. Dealing with high-level representations is beneficial to improving such clustering algorithms. The deep representations can be learned via linear mapping 
methods (e.g. Principal Component Analysis (PCA) and Non-negative Matrix Factorization (NMF) [162-164]) or non-linear mapping methods (e.g. Spectral Clustering [19, 165, 166], DAE [167-172], CNN [173] or DCAE [5, 174, 175]). We, here, focus on non-linear mapping methods, where features learned through deep networks provide an abstracted latent representation which is used for clustering analysis.

\subsubsection{Fundamental Structure for Deep Clustering}

\section{Representation Learning}

An auto-encoder [176] and a Restricted Boltzmann Machine network [177] are deep unsupervised models for representation learning that map inputs into new space representations, allowing useful features to be obtained through latent, hidden layers. A deep neural network is trained to optimize the parameters in order to learn the latent space function from the observed samples by minimizing the loss function. In this procedure, a deep neural network can discover better feature mappings. DAEs and DCAEs are commonly used methods for deep clustering. They can be better suited for a clustering problem [178], which can be solved in a short time for a small number of clusters, but might become an NP-hard problem for a large number of clusters.

\section{Clustering Loss Functions}

A loss function allows for evaluating how well a specific algorithm models the given data, which learns to reduce the error in predictions using an optimization function. The loss function of mean squared error is commonly used as an auto-encoder reconstruction loss to minimize the reconstruction error by the following optimization problem:

$$
\min \frac{1}{N} \sum_{n=1}^{N}\left\|x_{n}-\hat{x}_{n}\right\|^{2}
$$

where $N$ denotes the number of samples, $\hat{x}$ is a reconstructed image, and $x$ is an original image. Through this procedure, the input $x$ is mapped into a set of feature spaces, using the encoding part, from which the decoding part reconstructs the original data $\hat{x}$. This procedure allows auto-encoders to learn effective feature representations [179] or reduce the dimensionality of high-dimensional data [180] through mapping the data into a latent layer.

Although an auto-encode provides effective representations in a new latent space, it does not internally impose compact representation constraints using clustering. Clustering loss 40 
guides the clustering process to obtain more appropriate data partitioning. The clustering objective function [181] minimizes the distance between data samples and assigned centroids in a given space.

$$
\min \frac{1}{N} \sum_{n=1}^{N}\left\|\left(x_{n}\right)^{t}-c_{n}^{*}\right\|^{2}
$$

where $N$ denotes the number of samples, $\left(x_{n}\right)$ is the $n^{\text {th }}$ sample in the dataset at the $t^{\text {th }}$ iteration, and $c_{n}^{*}$ is the assigned cluster center to the $n^{\text {th }}$ sample. At each iteration, the clustering algorithm optimizes cluster centres $c$. At each iteration, each sample is assigned to the closest centroid $c_{n}^{*}=\arg \min _{c_{m}^{t-1}}\left\|\left(x_{n}\right)^{t}-c_{m}^{t-1}\right\|^{2}$. After that, the cluster centers are updated using the sample assignment computed in the previous iteration.

The joint deep clustering objective function is a combination of two parts: the first part is essentially the mean squared error minimizing the reconstruction error, Eq.(2.17), while the second part is the clustering objective function minimizing the distance between data representations in the latent space and their corresponding cluster centers, Eq.(2.18), producing a stable representation appropriate for the clustering process $[182,183]$. Similar to standard networks, the back-propagation method computes the gradient of the errors with respect to all parameters.

$$
\min _{W, b} \frac{1}{N} \sum_{n=1}^{N}\left\|x_{n}-\hat{x}_{n}\right\|^{2}+\lambda \cdot \frac{1}{N} \sum_{n=1}^{N}\left\|h^{t}\left(x_{n}\right)-c_{n}^{*}\right\|^{2}
$$

where $\lambda$ is a clustering weight-parameter that controls the contribution percentage of clustering cost function in the overall cost function, $h^{t}(*)$ is the internal representation obtained by the encoder mapping at the $t^{\text {th }}$ iteration, and $\left(x_{n}\right)$ is the $n^{\text {th }}$ sample in the dataset.

\subsubsection{Subsequent Multi-step Deep Clustering Methods}

These types of methods consist of two major steps which separate the clustering task from representation learning and feature extraction. In the first step, deep neural networks have been utilized to learn a lower-dimensional representation space and obtain useful features for clustering; after that, a clustering algorithm is applied to cluster the derived features in the second step.

Patel et al. [182] proposed kernel subspace clustering, which applies the procedure of learning a subspace by mapping input data into low-dimensional representations with an embedded projection. This strategy allows for learning the projection of data by minimizing the reconstruction error and finding the coefficients in the latent space for clustering to obtain better performance. 


\section{Background}

Huang et al. [169] made use of a DAE to support the clustering task. Their deep network learns low-dimensional representation from raw data, seeking to obtain high-level features used for clustering. After that, Kmeans is applied to cluster the obtained learned representation. Their work takes advantage of a deep neural network, which is used to acquire clusteringoriented representations by considering preserved locality and group sparsity constraints.

Tian et al. [168] also adopted the same approach. They proposed a graph clustering method based on a deep neural network which first learns a non-linear embedding of the original graph by a stacked auto-encoder, followed by the use of a Kmeans algorithm to perform clustering. The stacked auto-encoder is developed by stacking multiple autoencoders to make a deep neural network. Thus, they used a DAE to map the similarity graph into a low-dimensional space. Their study has compared auto-encoder and spectral clustering [19], and shown that the clustering performance is efficiently improved by replacing the eigenvalue decomposition with a DAE.

\subsubsection{Closed-loop Multi-step Deep Clustering Methods}

This approach consists of two salient steps, although such schemes take advantage of a deep neural network, where the original data is mapped into a representative feature space followed by clustering analysis, and feature space learning and the clustering process are two separate procedures. These steps alternate in a loop where the objectives are not optimized jointly, and they require various emphases, which can be time-consuming.

Xie et al. [171] also proposed Deep Embedded Clustering (DEC), which uses autoencoders as an initialization method to learn feature representations, and then perform clustering in an indirect way using DNNs. Their method fundamentally relies on pre-training the parameters of DAE, applying a loss function by optimizing a Kullback Leibler (KL) divergence objective to enforce a self-training target distribution. To perform clustering, the optimized DAE is pre-trained, and the learned features are fed into the Kmeans algorithm to cluster data points into their identical centroids. This process is repeated until clusters reach high confidence assignments. The clustering procedure does not directly contribute to the overall loss function, enabling a faster process.

Lia et al. [174] utilized a DCAE to learn feature representations. Their experiment attempted to train DCAE directly in an end-to-end fashion. Their method improves DEC [171] by replacing a stacked auto-encoder with a DCAE. The model utilizes the convolutional encoder-decoder network for fast and coarse image feature extraction. The decoder part is then 
neglected, and a soft Kmeans algorithm is added on top of the encoder part to form a unified clustering model. One of the shortcomings of this approach is that local structure preservation is ignored when optimizing the clustering task.

Guo et al. [175] also proposed a clustering method with DCAE. First, they prepared a DCAE network with a clustering layer utilizing KL-divergence to enforce a target distribution and then trained a DCAE model to learn feature representation. Through an iterative process, they fed the learned features into a Kmeans algorithm to cluster data points into their identical centroids. Similar to [171], the feature space learning and the clustering process are two separate procedures, and their clustering procedure does not contribute to the overall loss function. Moreover, KL-divergence may not be the most effective method for clustering, as it is not symmetric and hence, the distance from data point A to data point B is not the same as from B to A [184]. This can directly influence the quality of clusters generated.

Aljalbout et al. [185] introduced another closed-loop approach whereby auto-encoder training and Kmeans clustering are performed in an iterative loop. Their deep network initially learns representations to obtain high-level features, and the learned representations are then fed to Kmeans clustering. Similary, Yang et al. [183] applied a multi-step procedure in a closing-loop, applying deep convolutional networks to obtain more efficient latent representations and then applying Kmeans to perform clustering. Image clustering is conducted in the forward pass, while representation learning is carried out in the backward pass. Their aim was to make use of data representations to support clustering, and use the clustering results to provide supervisory signals when learning representations.

\subsubsection{Joint Deep Clustering Methods}

Deep neural networks with embedded clustering which simultaneously allow for the extraction of features and the clustering of assignments within the training process have been developed. The approach is performed by simultaneously optimizing the deep network parameters and the clustering parameters with joint loss functions in a combination of two parts: the reconstruction error and the clustering loss function.

Pioneering research by Song et al. [167] involved developing embedded clustering in a DAE framework. Their clustering process can be carried out simultaneously considering data reconstructions. They prepared the DAE network to be used for clustering by utilizing an objective function that is embedded into an auto-encoder model, minimizing the reconstruction error and clustering distances between data representations and their corresponding centroids 


\section{Background}

in low-dimensional space. Their clustering algorithm depends on the constraint of the distance between data representations and their identical cluster centres. Tian et al. [186] also proposed a method to integrate clustering methods (like Kmeans and GMM) into DAE, which simultaneously learns feature representations and cluster assignment under the same framework. However, these methods involve a cumbersome, time-consuming process in which they pertained the parameters of DAE to fine-tune the model of DAE-based data clustering. In other words, the optimized parameters of the DAE are copied into a new model to be used for a clustering framework. The DAE might also not be the best choice when clustering images, so embedding a clustering approach into a deep network that is more appropriate to image processing tasks may present an effective solution.

\subsubsection{Clustering Supervision}

Providing varying degrees of supervision to clustering methods has received considerable attention as a way of enhancing the performance of an unsupervised clustering task. Supervision can be derived from supervising knowledge through labeled data (i.e. by utilizing labeled data) or by enhancing human supervision through iterative user feedback [187-190]. Cohn et al. [187], for instance, introduced a semi-supervised clustering method that is iteratively based on user feedback to a clustering algorithm, which enhances human supervision to a clustering method. Here, we focus on data-driven supervision, where additional information about targeted groups is available.

Several approaches have been developed to study semi-supervised clustering, making use of partial supervision for the purpose of clustering guidance. The core aim is to provide an element of supervision to the clustering process, which can guide a clustering algorithm to obtain more enhanced discriminative groups and support its performance. The developed methods have attempted to cluster a large amount of unlabeled data in the presence of a small amount of supervision. Providing additional information can support the achievement of such a clustering task and help obtain more appropriate data partitioning. Basu et al. [191] explored the effect of using a small amount of labeled data to generate initial seeds for Kmeans. Moreover, Pedrycz et al. [192] proposed a fuzzy clustering algorithm with partial supervision which aims to take advantage of the available classification information and apply it actively as part of the optimization procedure. Their method allows structure to be found in the presence of a small amount of supervised data or labeled patterns. Others utilize a pairwise constrained clustering method [193] as a semi-supervised learning procedure for clustering algorithms, a framework 
which contains pairwise must-link and cannot-link constraints between points in a dataset. This semi-supervised approach emphasizes that two data points must be part of, or not part of, the same cluster, and has been applied to partitioning clustering methods [193,194], a hierarchical clustering method [195], and a density-based clustering method [196].

Supervised clustering approaches represent another clustering approach that involves a supervisory scheme in the clustering process, aiming to improve unsupervised clustering algorithms by exploiting the supervised information [197]. Pedrycz et al. [198] introduced a fuzzy supervised clustering algorithm that is carried out in the presence of labeled patterns. Their aim was to form a kind of structure that reconciles the structure discovered by the clustering mechanism and the labels of the patterns. Tagliaferri et al. [199] also employed supervised fuzzy clustering to search for the centroids of the hidden units, while Eick et al. [200,201] proposed a supervised clustering method which supposes that all obtained clusters hold ground truth labels, aiming to identify class-uniform clusters. Later, Al-Harbi et al. [202] introduced a supervised clustering method by modifying the Kmeans algorithm to be used as a classifier. They substantially used the labeled data for cluster seeding. Supervision can be exploited in pre-processing approaches for the traditional clustering context. Ismaili et al. [203] also studied the effectiveness of using supervised pre-processing steps for standard clustering to obtain better performance. A review paper by Amalaman et al. [204] focused on supervised taxonomy, which leverages background information such as class labels and distance metrics in order to enable capturing class-uniform regions in a dataset.

Although conventional semi-supervised and supervised clustering approaches have received much attention, with the revolution of deep learning, limited attention has been paid to semi-supervised and supervised deep clustering methods, where a clustering algorithm can deal with more enhanced discriminative latent features supported by supervision knowledge. The existing methods provide different ways to help clustering through levels of supervision, but most of the focus has been on modifying clustering methods. Making use of deep learning along with supervision can strengthen and support the learned features, and thus facilitate the job of traditional clustering methods in demonstrating satisfying performance.

\subsection{Deep Neural Network Compression and Acceleration}

As a result of increasing amounts of data and advanced computing power, deep learning models have turned into wider and deeper architectures, driving state-of-the-art performance in a wide range of applications. Despite their great success, deep networks often possess a vast num- 


\section{Background}

Table 2.1: Summary of Modern CNNs with their performance, computational and parameter complexities in ImageNet database. M/B indicates million/billion $\left(10^{6} / 10^{9}\right)$, respectively.

\begin{tabular}{|c|c|c|c|c|c|c|c|c|c|c|c|c|}
\hline & & \multicolumn{2}{c|}{ Performance } & \multicolumn{2}{c|}{ Computational complexity } & \multicolumn{2}{c|}{ Parameter complexity } \\
\hline Year & Network & layers(\#) & Size & Top-1 (\%) & Top-5 (\%) & FLOPs & Conv (\%) & FC (\%) & Par.(\#) & Conv (\%) & FC (\%) \\
\hline 2012 & AlexNet [9] & 8 & 240 megabyte & 36.70 & 15.30 & $724 \mathrm{M}$ & 91.9 & 8.1 & $61 \mathrm{M}$ & 3.8 & 96.2 \\
\hline 2014 & VGGNet [10] & 16 & 528 megabyte & 23.70 & 6.80 & $15.5 \mathrm{~B}$ & 99.2 & 0.8 & $138 \mathrm{M}$ & 10.6 & 89.4 \\
\hline 2014 & GoogleNet [42] & 22 & 88 megabyte & 22.10 & 6.30 & $1.6 \mathrm{~B}$ & 99.9 & 0.1 & $6.9 \mathrm{M}$ & 85.1 & 14.9 \\
\hline 2015 & ResNet [11] & 50 & 98 megabyte & 20.74 & 5.25 & $3.9 \mathrm{~B}$ & 100 & 0 & $25.5 \mathrm{M}$ & 100 & 0 \\
\hline
\end{tabular}

ber of parameters, and their significant redundancy in parameterization has become a widelyrecognized property [22]. The over-parametrized and redundant nature of deep networks cause expensive computational costs and high storage requirements, significant challenges which restrict many deep network applications. For example, to classify a single image, the VGG-16 model [10] requires more than 30 billion floating point operations per second (FLOPs) and contains about 138 million parameters with more than 500MB of storage space.

Most of the computational complexity originates in the convolutional layers due to massive multiplication and addition operations, although they contain less parameters due to parameter sharing. The number of FLOPs is utilized as a popular metric to estimate the complexity of CNN models. The FLOPs in convolutional layers are calculated as follows [48]:

$$
\text { FLOPs }=2 \mathrm{HW}\left(\mathrm{C}_{i n} \mathrm{~K}^{2}+1\right) \mathrm{C}_{\text {out }},
$$

where $H, W, C_{\text {out }}$ refers to the height, width and number of channels in the output tensor, $K$ is the kernel size, $C_{i n}$ denotes the number of input channels, and 1 is the corresponding bias. In contrast, most of the weights parameters exist in fully-connected layers, where the dense vector-matrix multiplications are very substantial resources. Table 2.1 represents the complexity of several CNNs' architectures, which consist of two parts: (1) the computational complexity is essentially related to the convolutional layers and (2) the parameters in fullyconnected layers dominate complexity. Accordingly, reducing the computational complexity of the convolutional layers became the focus of most model acceleration methods, while model compression methods mainly target the parameters of the fully-connected layers.

These complexities present significant challenges and restrict many applications. For instance, deploying sizeable deep learning models to a resource-limited device leads to various constraints as on-device memory is limited [205]. Therefore, reducing computational costs and storage requirements is critical to widen the applicability of deep learning models in a broader range of applications (e.g. mobile devices, autonomous agents, embedded systems, and 
real-time applications). Reducing the complexity of models while maintaining their powerful performance creates unprecedented opportunities for researchers to tackle major challenges in deploying deep learning systems to a resource-limited device. Network pruning focuses on discarding unnecessary parts of neural networks to reduce the computational costs and memory requirements associated with deep models. Pruning approaches have received considerable attention as a way to tackle over-parameterization and redundancy. Consequently, over-parameterized networks can be efficiently compressed and allow for the acquisition of a small subset of the whole model, representing the reference model with fewer parameters [206]. There is no authoritative guide for choosing the best network architecture; a model may require a certain level of redundancy during model training to guarantee excellent performance [207]. Hence, decreasing the size of a model after training can be an effective solution.

Pruning approaches were conceptualized in the early 1980s and '90s, and can be applied to any part of deep neural networks [208-214]. Optimal Brain Damage (OBD) by LeCun et al. [210], and Optimal Brain Surgeon (OBS) by Hassibi et al. [211] are considered pioneering works of network pruning, demonstrating that several unimportant weights can be removed from a trained network with little accuracy loss. Due to expensive computation costs, these methods are not applicable to today's deep models. Obtaining a sub-network with fewer parameters without reducing accuracy is the main goal of pruning algorithms. The pruned version, a subset of the whole model, can represent the reference model at a smaller size or with a smaller number of parameters. Over-parameterized networks can therefore be efficiently compressed while maintaining the property of better generalization [44].

In this section, we present an overview of popular methods and review recent works on compressing and accelerating deep neural networks, which have received considerable attention from the deep learning community and have already achieved remarkable progress. The recently advanced approaches for deep networks' compression and acceleration presented here can be classified into three categories: pruning methods, quantization methods, and low-rank factorization methods. The types of compression methods discussed below are intended to provide an overview of popular related techniques used in our research.

\subsubsection{Pruning Methods}

This section illustrates approaches that have been proposed to prune non-informative parts from heavy, over-parameterized deep models, including weights (i.e. parameters or connections) and units (i.e. neurons or filters). The core of network pruning is eliminating unimpor- 


\section{Background}

tant, redundant, or unnecessary parts according to the level of importance. Pruning methods can be applied to pre-trained models or trained from scratch and are further categorized into two classes according to pruning level: weights level and units level. Weight-based pruning eliminates unnecessary, low-weight connections between layers of a neural network while unitbased methods remove all weight connections to a specific unit, where both income or outgoing weights are removed.

\subsubsection{Weight-Based Methods}

Several weight-based methods have been proposed to prune non-informative connections. Recently, Han et al. [215] introduced a pruning method to remove connections whose absolute values are smaller than a predefined threshold value calculated using the standard deviation of a layer's weights. The network is then retrained to account for the drop in accuracy. Although Han's framework received significant attention and has become a typical method of network pruning, it focuses on the magnitude of weights, relies on iterative pruning and finetuning, and requires a particular software/hardware accelerator not supported by off-the-shelf libraries. Moreover, the reliance on a predefined threshold is not practical and too inflexible for some applications.

Liu et al. [216] showed the possibility of overriding the retraining phase by random reinitialization before the retraining step, which delivers equal accuracy with comparable training time. Furthermore, Mocanu et al. [217] replaced the fully-connected layers with sparselyconnected layers by applying initial topology based on the Erdôs-Rényi random graph. During training, fractions of the smallest weights are iteratively removed and replaced with the new random weights. Applying initial topology allows for the finding of a sparse architecture before training; however, this requires expansive training steps and obviously benefits from iteratively random initialization.

Through an iterative pruning technique, Frankle et al. [43] found that over-parameterized networks contain small sub-networks (winning tickets) that reach test accuracy comparable to the original network. The obtained sparse network can be trained from scratch using the same initialization as the original model to achieve the same level of accuracy. Their core idea was to find a smaller architecture better suited to the target task at the training phase. In a follow-up study, Frankle et al. [218] found that pruning networks at initialization values does not work well with deeper architectures, and suggested setting the weights to those obtained at a given early epoch in training. Various extensions have been developed for further improvement and to 
experimentally analyze the existence of the lottery hypothesis in other types of networks [219222].

To overcome the weaknesses associated with unstructured pruning, strategies corresponding to group-wise sparsity-based network pruning have been explored. Wen et al. [23] proposed the Structured Sparsity Learning (SSL) method, which imposes group-wise sparsity regularization on CNNs, applying the sparsity at different levels of their structure (filters, channels, and layers) to construct compressed networks. Lebedev et al. [223] also employed group-wise sparsity regularization to shrink individual weights toward zero so they can be effectively ignored. Furthermore, Zhou et al. [224] incorporated sparsity constraints on network weights during the training stage, aiming to build pruned DNNs. Although this proved successful in such sparse solutions, it results in damage to the original network structure and there is still a need to adopt special libraries or use particular sparse matrix multiplication to accelerate the inference speed in real applications.

It can be argued that the use of weight-based methods suffers from certain limitations. The need to remove low-weight connections means that important neurons whose activation does not contribute enough due to low-magnitude income or outgoing connections could be ignored. Moreover, the overall impact of weight-based pruning on network compression is lower than neuron-based methods. Pruning a neuron eliminates entire rows or columns of the weight matrices from both the former and later layers connected to that neuron, while weightbased methods only prune the low-weight connections between layers. To process the resulting sparse weight-matrices, some methods also require a particular software/hardware accelerator that off-the-shelf libraries do not support. Despite these drawbacks, the weight-based methods can be applied in combination with unit-based methods to add extra compression value.

\subsubsection{Unit-based Methods (Neurons, Kernels and Filters)}

Unit-based methods represent a pruning approach proposed to eliminate the least important units. He et al. [225] developed a simple unit-based pruning strategy that involves evaluating the importance of a neuron by summing the output weights of each one, and eliminating unimportant nodes based on this. They also apply neuron-based pruning utilizing the entropy of neuron activation. Their entropy function evaluates the activation distribution of each neuron based on a predefined threshold, which is only suitable with a sigmoid activation function. Since this method damages the network's accuracy, additional fine-tuning is required to obtain satisfactory performance. 


\section{Background}

Srinivas et al. [226] also introduced a unit-based pruning method by evaluating the weights similarity of neurons in a layer. A neuron is removed when its weights are similar to that of another in its layer. Mariet et al. [227] introduced Divnet, which selects a subset of diverse neurons and merges similar neurons into one. The subset is selected based on activation patterns by defining a probability measure over subsets of neurons. As with others, these non-structured pruning methods require software/hardware accelerators that are unsupported by off-the-shelf libraries and a multi-step procedure to prune neurons.

Filter-level pruning strategies have been widely studied. The aim of these strategies is to evaluate the importance of intermediate units, where pruning is conducted according to the lowest scores. Li et al. [228] suggested such a pruning method based on the absolute weighted sum, and Liu et al. [229] proposed a pruning method based on the mean gradient of feature maps in each layer, which reflects the importance of features extracted by convolutional kernels. Other data-driven pruning methods have been developed to prune non-informative filters. For instance, Polyak et al. [230] designed a statistical pruning method that removes filters based on variance of channels by applying the feature maps activation variance to evaluate the critical filters. Unimportant filters can also be pruned according to the level of importance. Luo's [231] pruning method is based on the entropy of the channels' output to evaluate the importance of their filters, and prunes the lowest output entropy, while $\mathrm{Hu}$ et al. [232] evaluated the importance of filters based on the average percentage of zero activations (APoZ) in their output feature maps.

Furthermore, Luo et al. [207] proposed the ThiNet method, which applies a greedy strategy for channel selection. This prunes the target layer by greedily selecting the input channel with the smallest increase in reconstruction error. The least-squares approach is applied to indicate a subset of input channels which have the smallest impact to approximate the output feature map. These methods tend to compress networks by simply adopting straightforward selection criteria based on statistical information. However, dealing with an individual CNN filter requires an intuitive process to determine selective and semantically meaningful criteria for filter selection, where each convolution filter responds to a specific high-level concept associated with different semantic parts. The most relevant work is a CNN pruning method inspired by neural network interpretability. Yeom et al. [233] combined the two disconnected research lines of interpretability and model compression by basing a pruning method on layer-wise relevance propagation (LRP) [104], where weights or filters are pruned based on their relevance score.

It could be argued that compressing a network via a training process may provide more ef- 
fective solutions. Ding et al. [234] presented an optimization method that enforces correlation among filters to converge at the same values to create identical filters, the redundant of which are safely eliminated during training. He et al. [235] proposed a filter pruning method which prunes convolutional filters in the training phase. After each training epoch, the method measures the importance of filters based on L2 norm, and the least essential filters are set to zero. He et al. [236] later iteratively measured the importance of the filter by calculating the distance between the convolution kernel and the origin or the geometric mean based on which redundant kernels are identified and pruned during training. Liu et al. [237] trained an auxiliary network to predict the weights of the pruned networks and estimate the performance of the remaining filters. Moreover, Zhonghui et al. [238] applied a training objective to compress the model as a task of learning a scaling factor associated with each filter and estimating its importance by evaluating the change in the loss function. AutoPruner [239] embedded the pruning phase into an end-to-end trainable framework. After each activation, an extra layer is added to estimate a similar scaling effect of activation, which is then binarized for pruning. A significant drawback of iterative pruning is the extensive computational cost, and pruning procedures based on training iterations often change the optimization function and even introduce hyper-parameters which make the training more challenging to converge.

\subsubsection{Quantization Methods}

Network quantization is a deep network compression procedure in which quantization, low precision, or binary representations are used to reduce the number of bits when representing each weight. Typical deep networks apply floating point (e.g. 32-bit) precision for training and inference, which is accompanied by a dramatic increase in computational costs, memory and storage requirements. Several works [240-242] introduced low bit-width models with a high level of accuracy, considering both activation and weight quantization. In the parameter space, Gong et al. [243], and Wu et al. [205] applied Kmeans clustering on the weight values for quantization. As a result, the network weights are stored in a compressed format after completing the training process, which allows them to reduce storage requirements and computational complexity. 8-bit quantization of the parameters has been proved to achieve significant speedup with minimal accuracy loss [244]. Suyog et al. [245] showed that truncating all parameters to 16-bits can result in a significant reduction in memory usage and floating point operations without compromising accuracy.

Others have proposed to simultaneously prune and quantize the weights' magnitudes of a 


\section{Background}

trained neural network. Han et al. [246] iteratively eliminated the unnecessary weight connections and quantized the weights, which were then encoded to single-bit precision by applying Huffman coding for further compression. This achieved state-of-the-art performance with no drop in model accuracy. Soft weight-sharing [247] was also developed to combine quantization and pruning approaches in one retraining procedure. Chen et al. [248] introduced a HashedNets model that applied a random hash function on the connection weights to force the weights to share identical values, resulting in a reduction in the number of trainable parameters by grouping them into hash buckets. These pruning approaches typically generate connection pruning in CNNs. In advanced cases, 1-bit quantization is used to represent each weight. A number of binary-based methods exist to directly train networks with binary weights (i.e., BinaryNet [249], BinaryConnect [250], and XNORNetworks [240]), who shared the idea of learning binary weights or activation during the training process.

The disadvantages of binary networks include significant performance drops when dealing with larger CNNs, and they ignore the impact of binarization on accuracy loss. To overcome this, Hou et al. [251] employed a proximal Newton algorithm with a diagonal Hessian approximation to minimize the overall loss associated with binary weights, and Lin et al. [252] quantized the representations at each layer when computing parameter gradients, converting multiplications into binary shifts by enforcing the values of the neurons of power-of-two integers.

\subsubsection{Low-rank Factorization Methods}

Low-rank approximation (factorization) is applied to determine the informative parameters, applying matrix or tensor decomposition. A weight matrix is factorized into a product of two smaller matrices, performing a similar function to the original weight matrix. In deep CNNs, the greatest computational cost results from convolution operations, so compressing the convolutional layers would improve overall speedup and compression rate. Convolutional units can be viewed as a 4D tensor, as the fact that the 4D tensor consists of a significant amount of redundancy drives the idea of tensor decomposition, which is an effective way to eliminate redundancy.

Low-rank factorization has been utilized for model compression and acceleration to achieve further speedup and obtain small CNN models. Rigamonti et al. [253] post-processed the learned filters by employing a shared set of separable 1D filters to approximate convolutional filters with low-rank filters, and Denton et al. [254] used low-rank approximation and cluster- 
ing schemes to reduce the computational complexity of CNNs. Jaderberg et al. [255] suggested using different tensor decomposition schemes, achieving double speedup for a particular convolutional layer with little drop in model accuracy. Low-rank factorization has also been used to exploit low-rankness in fully-connected layers. Denil et al. [206] utilized a low-rank decomposition of the weight matrices which learned from an auto-encoder to reduce the number of dynamic parameters, while Sainath et al. [256] showed that low-rank factorization of the last weighting layer significantly reduces the number of parameters. Lu et al. [257] adopted SVD to composite the fully-connected layer, attempting to design compact multi-task deep learning architectures. Low-rank approximation is made in a layer-by-layer fashion: at each layer, the layer is fine-tuned based on a reconstruction objective, while keeping all other layers fixed. Following this approach, Lebedev et al. [258] applied the non-linear least-squares algorithm, a type of Canonical Polyadic Decomposition (CPD), to approximate the weight tensors of the convolution kernels. Tai et al. [259] introduced a closed-form solution to obtain results of the low-rank decomposition through training constrained CNNs from scratch. The Batch Normalization layer $(\mathrm{BN})$ is utilized to normalize the activations of the latent, hidden layers. This procedure has been shown to be effective in learning the low-rank constrained networks.

Low-rank factorization approaches are computationally expensive because they involve decomposition operations. They also cannot perform global parameter compression as low-rank approximation is carried out layer-by-layer [24]. Undertaking sufficient retraining is the only technique which can be used to achieve convergence when compared to the original model. Despite their downsides, these approaches can be integrated with conventional pruning methods to obtain more compressed networks for further improvement.

\subsection{Summary}

This chapter has discussed necessary background information in preparation for introducing the proposed methods in the following chapters. An overview of deep learning algorithms was provided, emphasizing the fundamental operations that comprise all convolutional networks' backbone. We have highlighted popular conventional clustering algorithms, identifying their shortcomings as the dimensionality goes higher. This was followed by an overview of deep clustering, where an insight was provided into the development of deep learning algorithms as methods of representation learning used for the purpose of clustering. Moreover, we presented an overview of deep networks compressing and accelerating. Popular methods such as pruning methods, quantization methods, and low-rank factorization methods were described, as well as 


\section{Background}

advanced related techniques used in our research.

The following chapters will employ deep learning's ability to deal with high-level representations, making use of learned representations to enhance unsupervised learning and evaluate the characteristic strength of internal representations to compress and accelerate deep neural networks. Chapter 3 first presents DeepCluster, a clustering approach embedded in a deep convolutional auto-encoder. In Chapter 4, a new version of the DeepCluster model is introduced to include varying degrees of discriminative power, introducing a mechanism to allow for the imposition of regularization techniques and the involvement of a supervision component. Following this, the use of representation learning in deep network compression is explored, introducing two pruning frameworks in Chapters 5 and 6. Finally, Chapter 7 introduces a founding contribution to the area of applying deep time-series clustering (DTSC), identifies state-of-the-art, and presents an outlook of the field of DTSC from five important perspectives. 


\section{Chapter 3}

\section{DeepCluster: A Deep Convolutional Auto-encoder with Embedded \\ Clustering}

\section{Contents}

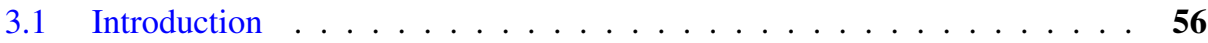

3.2 Proposed Methods . . . . . . . . . . . . . . . . . . . . 57

3.2.1 Deep Convolutional Auto-encoder (DCAE) _ . . . . . . . . 58

3.2.2 DeepCluster: A Deep Convolutional Auto-encoder with Embedded Clustering . . . . . . . . . . . . . . . 59

3.3 Experiments and Discussion . . . . . . . . . . . . . . 62

3.3.1 Datasets . . . . . . . . . . . . . . . . . . . . 62

3.3.2 Network Architectures . . . . . . . . . . . . . . . . 63

3.3.3 Evaluation Metrics $\ldots \ldots \ldots \ldots \ldots$

3.3.4 Implementation Details . . . . . . . . . . . . . . . . . 65

3.3.5 Baseline Methods . . . . . . . . . . . . . . . . . . . . . 66

3.3.6 Quantitative Results and Analysis _ . . . . . . . . . . . . . 66

3.3.7 Visualization Results and Analysis . . . . . . . . . . . . . 68

3.4 Summary . . . . . . . . . . . . . . . . . 73 


\subsection{Introduction}

Clustering is an unsupervised machine learning approach which aims to group a set of unlabeled data in the given feature space based on their homogeneous patterns. Data instances in one cluster are more homogeneous and similar to one another, while data instances that are different or far away from each other should be in different clusters. Traditional clustering algorithms attain a limited performance as the dimensionality increases. Therefore, dealing with high-level representations provides beneficial components that can support the achievement of such a clustering algorithm. As there is no supervision knowledge to provide information on categorical labels, representative features with compact clusters are much more valuable. They allow a clustering algorithm to obtain characteristic features and extract useful information for its structure.

Deep auto-encoder (DAE) [180] and deep convolutional auto-encoder (DCAE) are unsupervised models for representation learning. They map inputs into new space representations, allowing us to obtain useful features through encoding procedures. The data is projected into a set of feature spaces, using the encoding part, from which the decoding part reconstructs the original data. The training is performed in an unsupervised manner by minimizing the differences between the original data and reconstructed data with distance metrics. The major difference between DAE and DCAE is that the former adopts fully-connected layers to globally reconstruct a signal, while the latter utilizes local information to achieve the same objective. DCAEs may be better suited for image processing as they fully utilize the properties of convolutional neural networks (CNNs), which are shown to outperform all other techniques used on image data [83]. These methods have been exploited for the purpose of clustering, where features learned through deep networks (e.g. DAE [167-169,171] or DCAE [5,174,175]) provide an abstracted latent representation which is efficiently adopted for clustering analysis.

In this chapter, we propose clustering approaches embedded into a DCAE framework, which can learn feature representation and cluster assignment alternately. In contrast to conventional clustering approaches, our method makes use of representation learning by deep neural networks, which assists in finding compact and representative latent features for further recognition tasks. It also exploits the strength of DCAE to learn useful properties of image data for the purpose of clustering. We introduce two deep clustering methods: DCAE-Kmeans and DCAE-GMM. The proposed methods embed Kmeans and GMM clustering algorithms into a DCAE framework. Moreover, they introduce a general and flexible framework that can integrate different deep learning frameworks and traditional clustering methods via this joint 
optimization procedure. This procedure enables the classification of a data point into its identical cluster in the latent space, in a joint cost function by alternately optimizing the clustering objective and the DCAE objective, thereby producing stable representations which are appropriate for the clustering process. Most of the existing deep clustering methods fundamentally rely on the pre-training of parameters using different settings. However, our proposed method is trained in an end-to-end way using fixed settings without any pre-training or fine-tuning procedures, enabling a faster training process. Our work also differs from other approaches in terms of cost functions, architecture, and optimization. We evaluate our proposed method on three different image datasets: MNIST, USPS, and MNIST fashion, and compare our method with several baselines. We show that our approach substantially outperforms others in both reconstruction and clustering quality.

The rest of this chapter is organized as follows: in section 3.2, we present our proposed methodology, and outline our experimental results in section 3.3, providing qualitative and quantitative evaluations of proposed methods. Finally, concluding remarks and summary are provided in section 3.4.

\subsection{Proposed Methods}

The proposed approach embeds clustering algorithms (e.g. Kmeans and GMM) into a DCAE framework which is jointly optimized and trained in a fully unsupervised manner. The methods alternately learn effective feature representation and cluster assignment through DCAE. We initially give insight into the Deep Convolutional Auto-encoder (DCAE) and explain how it works in section 3.2.1, after which we introduce our DeepCluster method, explaining how it has been utilized for our clustering approaches in section 3.2.2. More specifically, we introduce the DCAE-Kmeans, where a Kmeans clustering algorithm is embedded into a DCAE framework in section 3.2.2.1. We then describe DCAE-GMM, where GMM clustering is embedded into DCAE, in section 3.2.2.2. The DeepCluster consists of two objective functions; one minimizes the reconstruction error, while the other minimizes the clustering objective. Both objectives are alternately optimized. Through this procedure, the model maps a 2D input image via a series of convolutional layers into latent representations. Then, deconvolutional layers are employed to reconstruct the data representation into its original shape. An effective representation, via the internal code, can be exploited to support our clustering task. The following subsections clarify our methodology. 


\subsubsection{Deep Convolutional Auto-encoder (DCAE)}

In contrast to the deep auto-encoder (DAE) model, which uses fully-connected layers, DCAE [85] uses convolutional and deconvolutional layers. The latter is more appropriate for image-processing tasks because it takes advantage of the convolutional neural networks' (CNN) properties [84]. These properties, mainly local connection and parameter sharing, distinguish CNN to have a property in translation latent features. In the encoding parts, convolutional layers are used as feature extractors to learn features by mapping the data into an internal layer. A latent representation of the $n^{\text {th }}$ feature map of the existing layer is given by the following form:

$$
h^{n}=\sigma\left(x * W^{n}+b^{n}\right)
$$

where $W$ is the filters and $b$ is the corresponding bias of the $n^{\text {th }}$ feature map, $\sigma$ is the activation function (e.g. sigmoid, ReLU), and $*$ denotes the $2 \mathrm{D}$ convolution operation.

In contrast, the deconvolutional layers invert this process and reconstruct the latent representation back into its original shape; thus, this process maps the obtained features into pixels [87] by using the following form:

$$
y=\sigma\left(\sum_{n \in H} h^{n} * \tilde{W}^{n}+c\right),
$$

where $H$ denotes the group of latent feature maps, $\tilde{W}$ is the flip operation over both dimensions of the weights, $c$ is the corresponding bias, $\sigma$ is the activation function, and $*$ denotes the $2 \mathrm{D}$ convolution operation. The difference between convolution operations in Eq.(3.1) and Eq.(3.2) is that the convolutional layer performs a valid convolution, which decreases the output size of feature maps, while the deconvolution layer performs a full convolution, which increases the output size of feature maps [85,88]. In other words, if $x$ is an $m \times m$ image and the filters are $n \times n$, then the valid convolution performs $(m-n+1) \times(m-n+1)$ and full convolution performs $(m+n-1) \times(m+n-1)$.

The DCAE extracts latent representations through its internal layer by minimizing the reconstruction error; the euclidean (L2) loss function is utilized in this case. Similar to standard networks, the back-propagation method computes the gradient of the error with respect to all parameters.

$$
E_{1}=\frac{1}{N} \sum_{n=1}^{N}\left\|x_{n}-\hat{x}_{n}\right\|^{2}
$$

where $\hat{x}$ is a reconstructed image, and $x$ is an original image. 


\subsubsection{DeepCluster: A Deep Convolutional Auto-encoder with Embedded Clustering}

Taking advantage of the strength of the DCAE model described in section 3.2.1, we use it as a training procedure for feature transformations. The goal of our clustering method is to learn feature representation and cluster assignment alternately. Using DCAE as a feature extractor supports the achievement of such a clustering process. This allows the clustering method to deal with learned features instead of raw data. Here, we develop two deep clustering methods. Although DCAE provides effective representation in a new latent space, it does not internally impose compact representation constraints using clustering. Therefore, we introduce two objective clustering functions to the DCAE model.

\subsubsection{Embedding Kmeans clustering into DCAE (DCAE-Kmeans)}

We append an objective clustering function to the DCAE model, aiming to find the closest cluster centre for each instance and assign it to that cluster with great confidence. The objective clustering function minimizes the distance between data samples and assigned centroids in latent space:

$$
E_{2}=\lambda \cdot \frac{1}{N} \sum_{n=1}^{N}\left\|h^{t}\left(x_{n}\right)-c_{n}^{*}\right\|^{2}
$$

where $N$ denotes the number of samples, $\lambda$ is the clustering weight-parameter that controls the contribution percentage of clustering cost function in the overall cost function Eq.(3.5), $h^{t}(*)$ is the internal representation obtained by the encoder mapping at the $t^{\text {th }}$ iteration, $\left(x_{n}\right)$ is the $n^{\text {th }}$ sample in the dataset, and $c_{n}^{*}$ is the assigned cluster center to the $n^{\text {th }}$ sample. The overall cost function is a combination of two parts: the first part is essentially the Euclidean (L2) loss function minimizing reconstruction error, by Eq.(3.3), while the second part is the clustering objective function minimizing the distance between data representation in a latent space and their corresponding cluster centres, by Eq.(3.4).

$$
\min _{W, b} E_{1}+E_{2}
$$

At each epoch, our model optimizes two components using an optimizer and backpropagation algorithm: (1) conventional auto-encoder parameters as well as mapping function $h$, and (2) cluster centres $c$. At each epoch, the model optimizes the mapping function $h$, while keeping the cluster centres fixed at $c$. Thereafter, each obtained new internal representation is 
assigned to the closest centroid; this is defined as:

$$
c_{n}^{*}=\arg \min _{c_{m}^{t-1}}\left\|h^{t}\left(x_{n}\right)-c_{m}^{t-1}\right\|^{2},
$$

where $c_{m}^{t-1}$ denotes the cluster centres computed at the previous epoch. After each internal representation is assigned to the closest cluster centre, the cluster centres are updated using the sample assignment computed in the previous epoch via the following equation:

$$
c_{m}^{t}=\frac{\sum_{x_{n} \in c_{m}^{t-1}} h^{t}\left(x_{n}\right)}{\sum c_{m}^{t-1}},
$$

where $c_{m}^{t-1}$ represents all samples that belong to the $m^{\text {th }}$ cluster at the previous epoch, and $\sum c_{m}^{t-1}$ is the number of samples that belong to the $m^{\text {th }}$ cluster. The learning algorithm of DCAE-Kmeans is given in Algorithm 1.

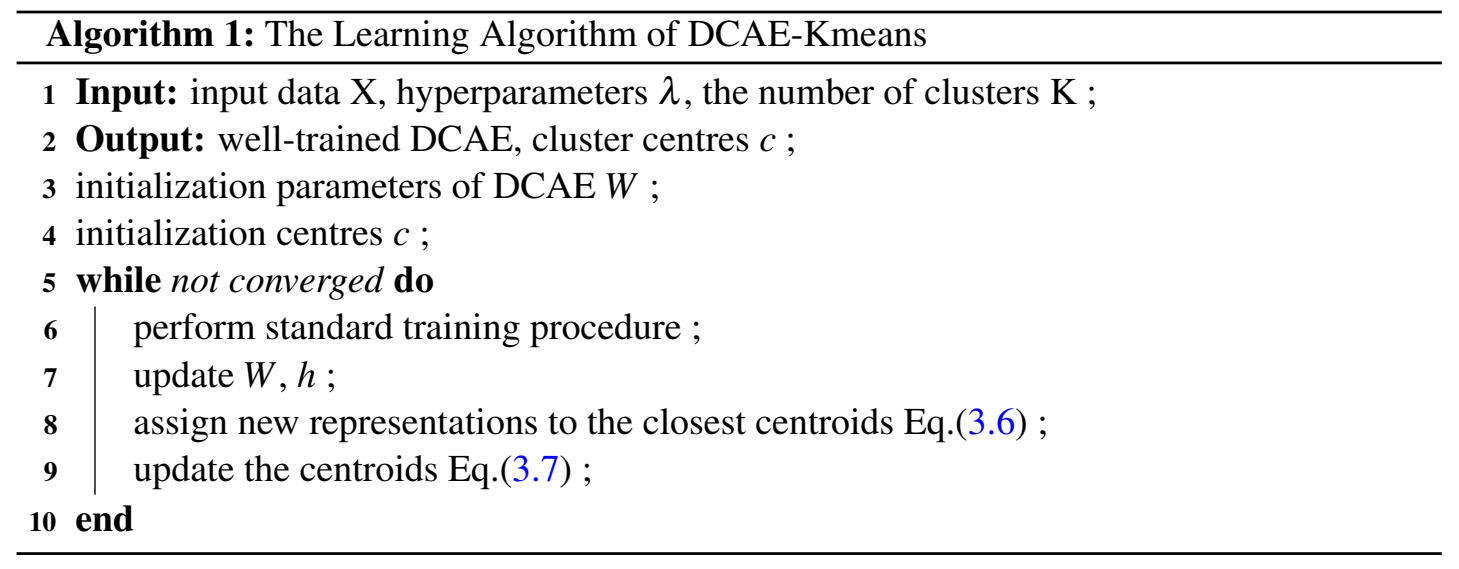

\subsubsection{Embedding Gaussian mixture modelling (GMM) clustering into DCAE (DCAE-GMM)}

We also embed a model-based clustering method into the DCAE. GMM is a model-based approach to data clustering, which is described as a weighted sum of single Gaussian functions. GMM is a probabilistic model that is described by several parameters and involves a mixture of multiple Gaussian functions, all of which allow for the learning of the distribution of data in the latent space. Therefore, all data samples are assumed to be generated from a mixture of a number of Gaussian distributions with unknown parameters. GMM differs from Kmeans in various ways. For instance, instead of describing each cluster by only its centroid, GMM, as a probabilistic approach, represents a cluster by its centroid (mean) $\mu$, standard deviation 
(covariance matrix) $\Sigma$ for each Gaussian function, which can be conveniently computed from clustered feature representations, and the size of the cluster (weight) $P$, which is computed by counting the amount of data belonging to each Gaussian component. GMM also performs soft clustering, whereas Kmeans performs hard clustering.

Here, we embed a cost function to fit a GMM to the latent features of DCAE. As a clustering objective function, the log posterior (likelihood) probability of the learned features is defined as follows [186]:

$$
E_{3}=\lambda \cdot \sum_{n=1}^{N} \ln \left[\sum_{k=1}^{K} \pi_{k} \mathscr{N}\left(h^{t}\left(x_{n}\right) \mid \mu_{k}, \Sigma_{k}\right)\right],
$$

where $\lambda$ is the clustering weight parameter that controls the contribution percentage of the clustering cost function in the overall cost function Eq.(3.9), which defines a trade-off between the network objective and the clustering objective. $N$ denotes the number of samples, while $K$ denotes the number of components (Gaussians). $\mathscr{N}\left(h^{t}\left(x_{n}\right) \mid \mu_{k}, \Sigma_{k}\right)$ is a multivariate Gaussian distribution of $h^{t}(*)$, which is the internal representation obtained by the encoder mapping at the $t^{\text {th }}$ iteration. $\mu_{k}$ is a mean, $\Sigma_{k}$ is a covariance matrix, and $\pi_{k}$ is a mixing coefficient of the $k^{\text {th }}$ component of our Gaussians.

The overall cost function is a combination of two parts: the first part is essentially the Euclidean loss (L2) minimizing reconstruction error, by Eq.(3.3), while the second part is the clustering objective function, by Eq.(3.8).

$$
\min _{W, b} E_{1}+E_{3} .
$$

At each epoch, our model optimizes several components using an optimizer and backpropagation: DCAE parameters, as well as mapping function $h$, and GMM parameters $\mu_{k}, \Sigma_{k}$, and $\pi_{k}$. Here, we mainly use the iterative Expectation-Maximization (EM) algorithm to estimate GMM's parameter so that each data point can be represented by its probability. This process effectively allows for maximizing the log posterior probability. At each epoch, the model optimizes the mapping function $h$, while keeping the GMM parameters fixed at $\mu_{k}^{t-1}, \Sigma_{k}^{t-1}$, and $\pi_{k}^{t-1}$. After that, the posterior probability for each obtained new internal representation is computed; this is defined as:

$$
P\left(k \mid h^{t}\left(x_{n}\right)\right)=\frac{\pi_{k}^{t-1} \mathscr{N}\left(h^{t}\left(x_{n}\right) \mid \mu_{k}^{t-1}, \Sigma_{k}^{t-1}\right)}{\sum_{j=1}^{K} \pi_{j}^{t-1} \mathscr{N}\left(h^{t}\left(x_{n}\right) \mid \mu_{j}^{t-1}, \Sigma_{j}^{t-1}\right)} .
$$

The GMM parameters are then updated using the sample assignment computed in the previous epoch $t-1$ via the following equations: 


$$
\pi_{k}^{t}=\frac{1}{N} \sum_{n=1}^{N} P\left(k \mid h^{t}\left(x_{n}\right)\right),
$$

where $\pi_{k}^{t}$ is the new mixing coefficient, which is simply the normalized summation of the posterior probability. The posterior probability-weighted mean $\mu_{k}^{t}$ and the posterior probabilityweighted covariance matrix $\Sigma_{k}^{t}$ are updated as follows:

$$
\begin{gathered}
\mu_{k}^{t}=\frac{\sum_{n=1}^{N} P\left(k \mid h^{t}\left(x_{n}\right)\right) h^{t}\left(x_{n}\right)}{\sum_{n=1}^{N} P\left(k \mid h^{t}\left(x_{n}\right)\right)}, \\
\Sigma_{k}^{t}=\frac{\sum_{n=1}^{N} P\left(k \mid h^{t}\left(x_{n}\right)\left(h^{t}\left(x_{n}\right)-\mu_{k}^{t}\right)\left(h^{t}\left(x_{n}\right)-\mu_{k}^{t}\right)^{T}\right.}{\sum_{n=1}^{N} P\left(k \mid h^{t}\left(x_{n}\right)\right)} .
\end{gathered}
$$

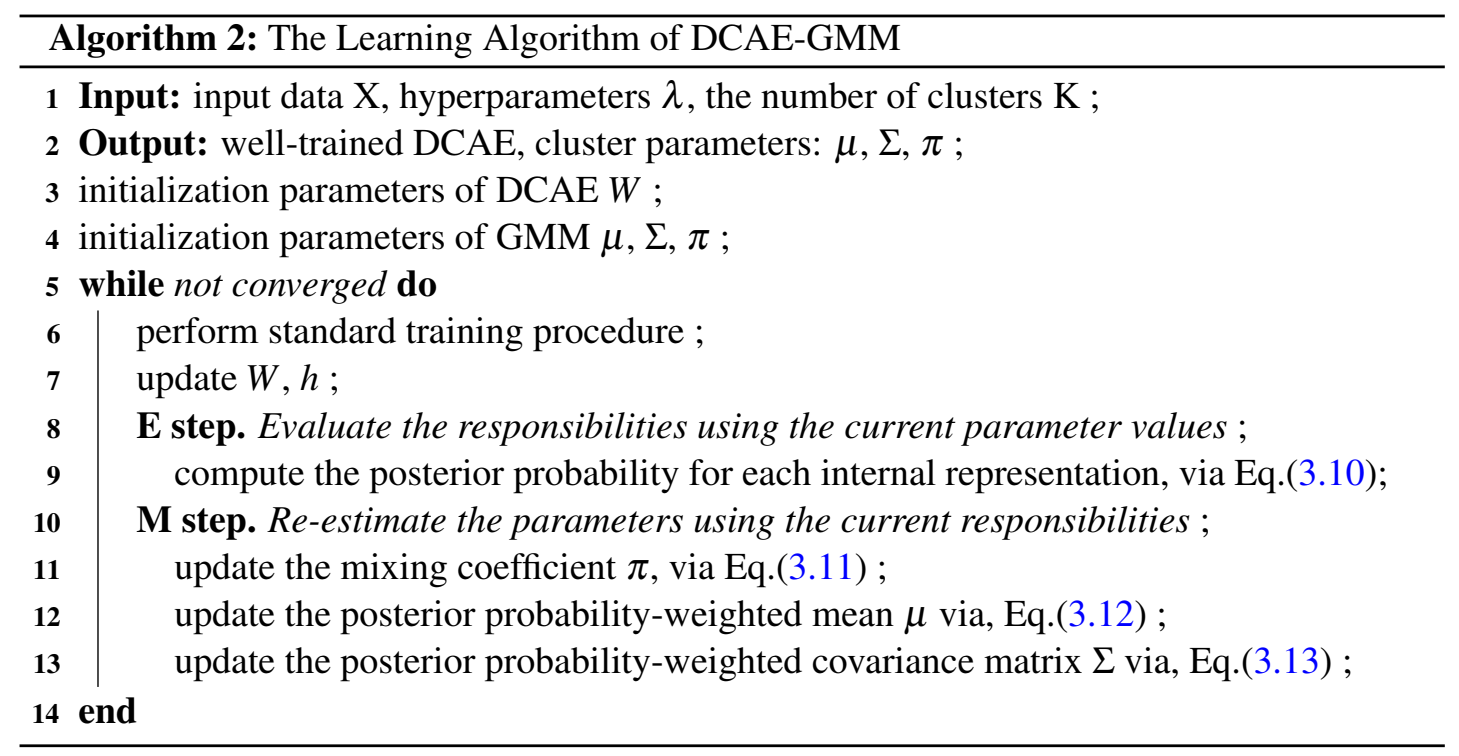

\subsection{Experiments and Discussion}

\subsubsection{Datasets}

We evaluated our proposed methods on three different image datasets, including MNIST, USPS, and MNIST-fashion, two of which are the most commonly used datasets in the area of deep clustering. Specifications of these datasets are presented in Table 3.1.

- MNIST [83]: consists of handwritten digits [0-9] images, and each example is a $28 \times 28$ grayscale image, associated with a label from 10 classes. MNIST comprises 60,000 examples as a training set and 10,000 examples as a test set. 
- USPS [260]: also consists of handwritten digits [0-9] images, and each example is a $16 \times 16$ grayscale image, associated with a label from 10 classes. Each class has 1,100 samples, and the total number of images in this dataset is 11,000 , which consists of 7,291 examples as a training set and 2,007 examples as a test set.

- MNIST-Fashion [261]: is article images. Each example is a $28 \times 28$ grayscale image associated with a label from 10 classes [T-shirt/top, Trouser, Pullover, Dress, Coat, Sandal, Shirt, Sneaker, Bag, Ankle boot]. MNIST-Fashion consists of 60,000 examples as a training set and 10,000 examples as a test set.

No modification has been applied to the input data; we have only normalized scale images from the range of $[0,255]$ to be in the range of $[0,1]$ on a per-image basis by using Eq.(3.14). The labels have only been used as ground truth to evaluate clustering results in the last stage.

$$
\hat{x}=\frac{x-\min (x)}{\max (x)-\min (x)} .
$$

Table 3.1: Details of datasets used in our experiments for the DeepCluster method.

\begin{tabular}{|r|c|c|c|c|c|}
\hline dataset & Examples & Classes & Image Size & Channels & Number of classes \\
\hline MNIST & 70000 & 10 & $28 \times 28$ & 1 & 10 \\
\hline USPS & 11000 & 10 & $16 \times 16$ & 1 & 10 \\
\hline MNIST-Fashion & 70000 & 10 & $28 \times 28$ & 1 & 10 \\
\hline
\end{tabular}

\subsubsection{Network Architectures}

The detailed architecture of the DCAE model is presented in Fig. 3.1. Our contributions to the architecture are the following: we exploit the learned features via the internal layer and feed them to the clustering loss function, which embeds a clustering algorithm into the body of a DCAE model, applying a joint cost function by jointly optimizing the clustering objective and the DCAE objective. Therefore, instead of optimizing an auto-encoder to reach optimal reconstruction, we sequentially optimize the mapping function $h$ and cluster parameters to obtain efficient clustering results.

The network architecture consists of three convolutional layers with different filter sizes (e.g. $5 \times 5$ and $4 \times 4$ ) based on the datasets. There are 32 kernels in the first convolutional layer, 64 kernels in the second convolutional layer, and 128 kernels in the third convolutional 


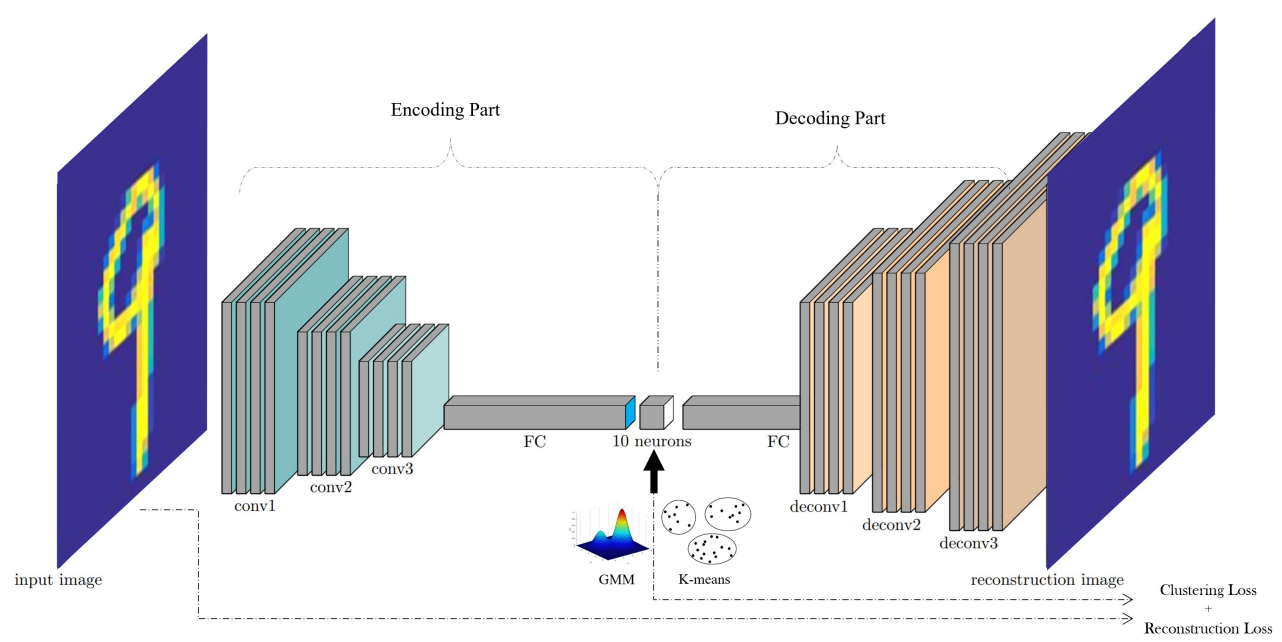

Figure 3.1: The architecture of the DeepCluster model.

Table 3.2: Detailed configuration of the DCAE network architecture used in the experiments.

\begin{tabular}{|c|c|c|c|}
\hline Layer & MNIST & USPS & MNIST Fashion \\
\hline Convolutional & $5 \times 5 \times 32$ & $4 \times 4 \times 32$ & $5 \times 5 \times 32$ \\
\hline Convolutional & $5 \times 5 \times 64$ & $4 \times 4 \times 64$ & $5 \times 5 \times 64$ \\
\hline Convolutional & $3 \times 3 \times 128$ & $2 \times 2 \times 128$ & $3 \times 3 \times 128$ \\
\hline Fully-Connected & 1152 & 512 & 1152 \\
\hline Fully-Connected & 10 & 10 & 10 \\
\hline Fully-Connected & 1152 & 512 & 1152 \\
\hline Deconvolutional & $3 \times 3 \times 128$ & $2 \times 2 \times 128$ & $3 \times 3 \times 128$ \\
\hline Deconvolutional & $5 \times 5 \times 64$ & $3 \times 3 \times 64$ & $5 \times 5 \times 64$ \\
\hline Deconvolutional & $5 \times 5 \times 32$ & $3 \times 3 \times 32$ & $5 \times 5 \times 32$ \\
\hline
\end{tabular}

layer. This is followed by two fully-connected layers, of which the second layer has 10 neurons as a result of the encoding part. In the decoding part, a single fully-connected layer is followed by three deconvolutional layers. The first deconvolutional layer consists of 128 kernels, the second consists of 64 kernels, and the third consists of 32 kernels. The detailed configuration of the DCAE network architectures used in the experiments for the three datasets is presented in Table 3.2. ReLU was utilized as a standard activation function, except the reconstruction layer, Sigmoid activation function was utilized. 


\subsubsection{Evaluation Metrics}

To justify our methods, two evaluation approaches are used to compute the cluster quality: Accuracy (ACC) and Normalized Mutual Information (NMI), which distinguish the clustering results generated by our DeepCluster method and the ground truth labels.

\subsubsection{Accuracy (ACC)}

Clustering accuracy is a widely used measurement to evaluate clustering results. It is computed using obtained clustering results and ground truth labels by using the following form $[262,263]$ :

$$
\text { Accuracy }=\frac{\sum_{i=1}^{n} \delta\left(y_{i}, \operatorname{map}\left(c_{i}\right)\right)}{n},
$$

where $N$ is the number of samples, $y_{i}$ denotes ground truth labels, $c_{i}$ is obtained clusters, $\delta(y, c)$ is a function that equals one if $y=c$ and zero otherwise, and $\operatorname{map}\left(c_{i}\right)$ is the permutation function that maps obtained cluster labels into their corresponding ground truth labels.

\subsubsection{Normalized Mutual Information (NMI)}

The NMI is another metric used to measure clustering quality. It is defined between two random variables as [264]:

$$
N M I(X ; Y)=\frac{I(X ; Y)}{\sqrt{H(X) H(Y)}},
$$

where $X$ denotes ground truth labels, $Y$ is the obtained cluster, $I(X ; Y)$ is the mutual information between $X$ and $Y$, and $H(X)$ and $H(Y)$ denote the utilized entropy, which normalize the value of mutual information into $[0,1]$ range.

\subsubsection{Implementation Details}

The proposed method was implemented using Keras [265] and TensorFlow [52] in Python. The model was trained end-to-end in an unsupervised manner. There are no pre-training and finetuning procedures involved. All weights were initialized using Xavier uniform initializer and cluster centres were also initialized randomly. The Adam optimizer [63] was used, where each batch contains 100 randomly shuffled images. We set $\lambda$, the clustering weight-parameter that controls the loss contribution percentage of clustering error, to 0.1 throughout all experiments, and the model converged after 500 epochs. Throughout our experiment, we used an initial learning rate of 0.001 , a momentum of 0.9 , and a weight decay of 0.0005 . 


\subsubsection{Baseline Methods}

To evaluate the cluster quality, the two evaluation metrics, accuracy (ACC) and normalized mutual information (NMI), were computed. We demonstrate the effectiveness of our clustering methods by comparing ours with seven baseline methods: Kmeans, GMM, DEC, AEC, DCKmeans, DC-GMM, and DCEC. Kmeans and GMM are applied to the raw input data. DEC by Xie et al. [171], AEC by Song et al., [167] and DC-Kmeans and DC-GMM by Tian et al., [186] are clustering methods that learn feature representations utilizing a deep auto-encoder, while $D C E C$, by Guo et al., [175] is a clustering method that learns feature representations utilizing a DCAE. These methods utilize deep networks to learn abstracted latent representations that are used for clustering analysis. The results are summarized in Table 3.3.

\subsubsection{Quantitative Results and Analysis}

On both ACC and NMI metrics, our proposed methods, DCAE-GMM and DCAE-Kmeans, outperform all baseline methods by a significant margin. Table 3.3 demonstrates that $D C A E$ GMM outperforms all other methods on all three datasets, where $96.78 \%$ and $92.14 \%$ were achieved on both ACC and NMI respectively on the MNIST dataset, $86.07 \%$ and $85.18 \%$ were achieved on both ACC and NMI respectively on the USPS dataset, and $62.95 \%$ and $72.41 \%$ were achieved on both ACC and NMI respectively on the MNIST-Fashion dataset. The results also demonstrate that on the MNIST dataset, DCAE-Kmeans outperforms the baseline methods by a significant margin on both ACC and NMI metrics, where $93.42 \%$ and $86.78 \%$ were achieved on both ACC and NMI respectively. Notably, the proposed method substantially outperforms the one in second place by $8.13 \%$ on MNIST and $4.25 \%$ on USPS on ACC, which also uses a DCAE approach with a clustering algorithm.

Table 3.3 also experimentally analyzes the performance of a clustering algorithm in different spaces, i.e., the original data space and space learned via non-linear mapping with both DAE and DCAE. The experimental results of traditional Kmeans and GMM support our hypothesis that conventional clustering algorithms attain a limited performance as the dimensionality increases. Deep neural networks are considered as a potential solution to overcoming this issue by allowing a clustering algorithm to deal with a clustering-friendly representation instead of high dimensional data. This high-level representation provides beneficial properties that can support traditional clustering algorithms in demonstrating satisfying performance. With AE space, the clustering algorithm performs much better compared to the original space clustering. The comparative results of DCAE-Kmeans and DCAE-GMM also demonstrate that 
Table 3.3: Comparison of clustering quality with the baselines on three datasets using two evaluation metrics: accuracy (ACC) and normalized mutual information (NMI).

\begin{tabular}{|r|c|c|c|c|c|c|}
\hline & \multicolumn{2}{|c|}{ MNIST } & \multicolumn{2}{c|}{ USPS } & MNIST-FASHION \\
\hline & ACC & MNI & ACC & MNI & ACC & MNI \\
\hline Kmeans & $61.63 \%$ & $57.26 \%$ & $61.63 \%$ & $57.26 \%$ & $48.30 \%$ & $51.47 \%$ \\
\hline GMM & $62.93 \%$ & $59.74 \%$ & $62.93 \%$ & $59.74 \%$ & $51.90 \%$ & $53.59 \%$ \\
\hline Xie et. al [171], DEC & $84.30 \%$ & $83 \% \%^{\star}$ & $76.2 \%$ & $76.7 \%$ & $51.8 \%$ & $54.6 \%$ \\
\hline Song et. al [167], AEC & $76.00 \%$ & $66.90 \%$ & $71.50 \%$ & $65.10 \%$ & - & - \\
\hline Tian et. al [186], DC-Kmeans & $80.15 \%$ & $74.48 \%$ & $64.42 \%$ & $57.37 \%$ & - & - \\
\hline Tian et. al [186], DC-GMM & $85.55 \%$ & $83.18 \%$ & $64.76 \%$ & $69.39 \%$ & - & - \\
\hline Guo et. al [175], DCEC & $85.29 \%$ & $83.61 \%$ & $79.00 \%$ & $82.57 \%$ & $56.60 \%$ & $61.40 \%$ \\
\hline \hline Proposed, DCAE-Kmeans & $93.42 \%$ & $86.78 \%$ & $83.25 \%$ & $82.22 \%$ & $58.20 \%$ & $67.02 \%$ \\
\hline Proposed, DCAE-GMM & $\mathbf{9 6 . 7 8 \%}$ & $\mathbf{9 2 . 1 4 \%}$ & $\mathbf{8 6 . 0 7 \%}$ & $\mathbf{8 5 . 1 8 \%}$ & $\mathbf{6 2 . 9 5 \%}$ & $\mathbf{7 2 . 4 1 \%}$ \\
\hline
\end{tabular}

- The original paper did not report their accuracy on the MNIST-FASHION dataset.

* The results are excerpted from [266].

the procedure of embedding clustering algorithms into DCAE leads to the formation of a kind of structure that enhances the achievement of deep clustering methods, which significantly improves the representation of data for clustering.

Comparing with all deep embedded clustering baselines, the experimental results in all datasets demonstrate that methods utilizing a DCAE to learn feature representations (i.e., our clustering methods and DCEC by Guo et al. [175]) perform much better than methods that utilize a standard auto-encoder (DAE). DCAE mainly takes advantage of convolutional neural networks' (CNN) properties to preserve the local structure of the data and share parameters which can be more appropriate for image-processing tasks. Under these advantages, the clustering algorithm can perform much better, and therefore improve its accuracy.

Fig. 3.2 shows the changes of both ACC and NMI with the number of training cycles in all experimental datasets, which indicate that clustering stably converges using an iterative training scheme. The performance is improved after dozens of iterations, which demonstrates that the convergence of our proposed method is fast and more stable. After a reasonable number of epochs, both ACC and NMI become stable, and are gradually improved until they converge. Therefore, we have reported the results after 500 iterations. 


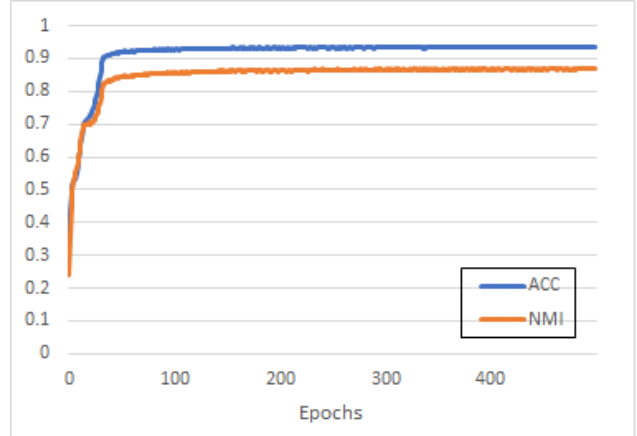

A. MNIST

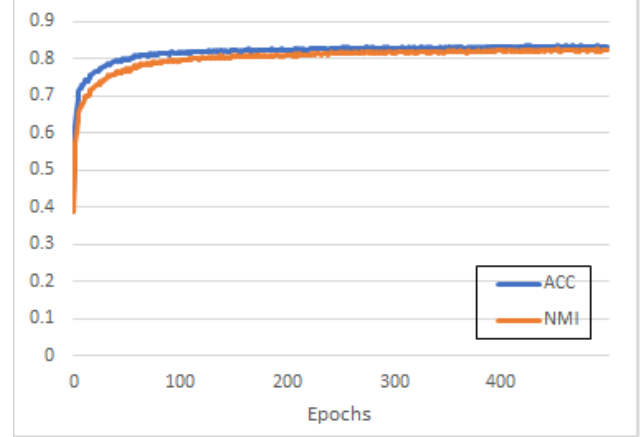

B. USPS

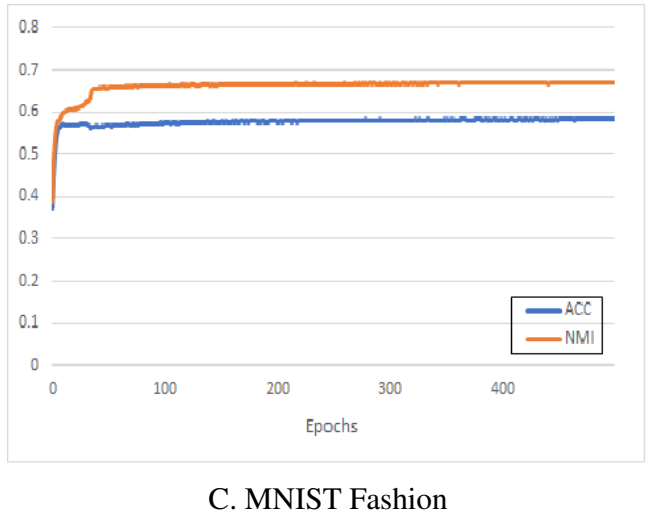

Figure 3.2: Changes in ACC and NMI of the DCAE-Kmeans proposed method over different epochs during training on 3 datasets.

\subsubsection{Visualization Results and Analysis}

We consider not only quantitative analysis but also visual analysis, as it provides a practical way in which to evaluate the effectiveness of our proposed method. The reconstruction quality of the DCAE is essential because it is highly desirable to obtain efficient clustering results as well as typical reconstruction quality. Obtaining identical reconstruction quality indicates that the DCAE model efficiently learns latent representations, which are considered as representative features of the input data. We have visualized original inputs and reconstruction images provided by our model, which allows us to visually differentiate and evaluate how well our model reconstructs original images. Fig. 3.3 shows the quality of the reconstructed images for random examples from 3 different datasets. Fig. 3.3 shows that our model tends to reach optimal reconstruction, and the original data points are retrieved as flawlessly as possible.

The DCAE is trained to transform the data into latent representations and then reconstruct 

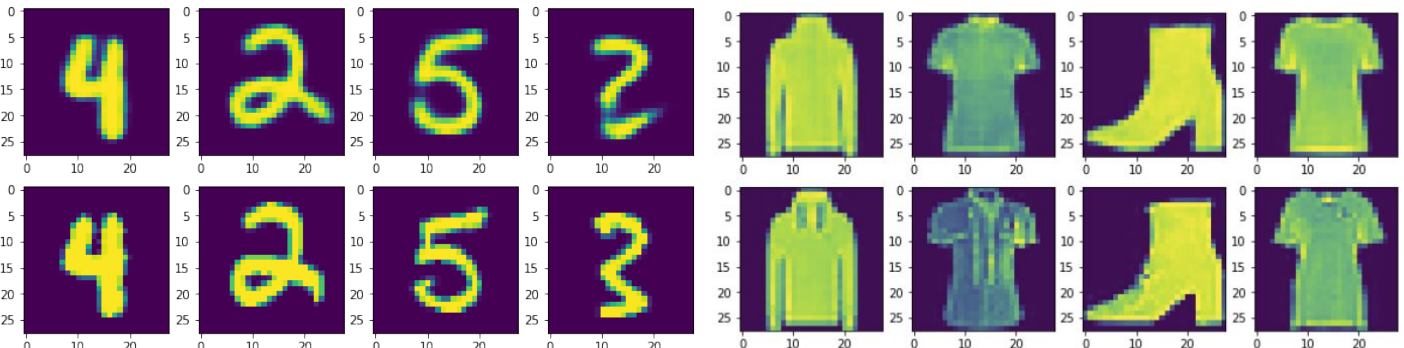

A. MNIST

B. MNIST Fashion

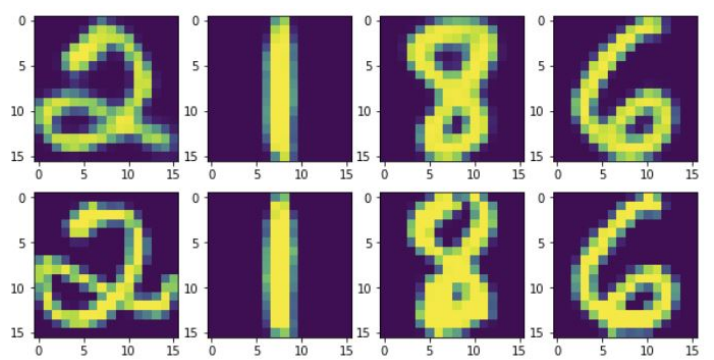

C. USPS

Figure 3.3: Visualizations of reconstruction images (Top) and input images (Bottom) on 3 datasets using the DCAE-Kmeans proposed method.

the original input or obtain an optimal approximation of the underlying data representation by minimizing the reconstruction error. Some examples of original inputs and reconstruction images obtained by our model are demonstrated in Fig. 3.3; they allow us to visually differentiate and evaluate the reconstruction quality of our model. In Fig. 3.3, the reconstructed images (top rows) look qualitatively identical to the original ones (bottom rows), with certain levels of blurring. This method is best suited for capturing common patterns rather than subtle details at local regions for reconstruction. One clear example can be seen in Fig. 3.3B.; the t-shirt reconstruction image shows the shape of the t-shirt with less focus on details at local regions. It is also worth noting that because the number 3 has a similar structure to that of the number 2 , particularly with regards to the upper part of both numbers (see Fig. 3.3A.), the model failed to reconstruct the obtained latent representation back to its original shape. One reasonable explanation is that the proposed method is designed for unsupervised representation learning with a signal reconstruction objective, where the information necessary to be able to distinguish between the two numbers (e.g. supervision information, which aids the learning of discriminative feature representations) is not available.

Furthermore, Fig. 3.4 presents the reconstruction of cluster centres in three different 


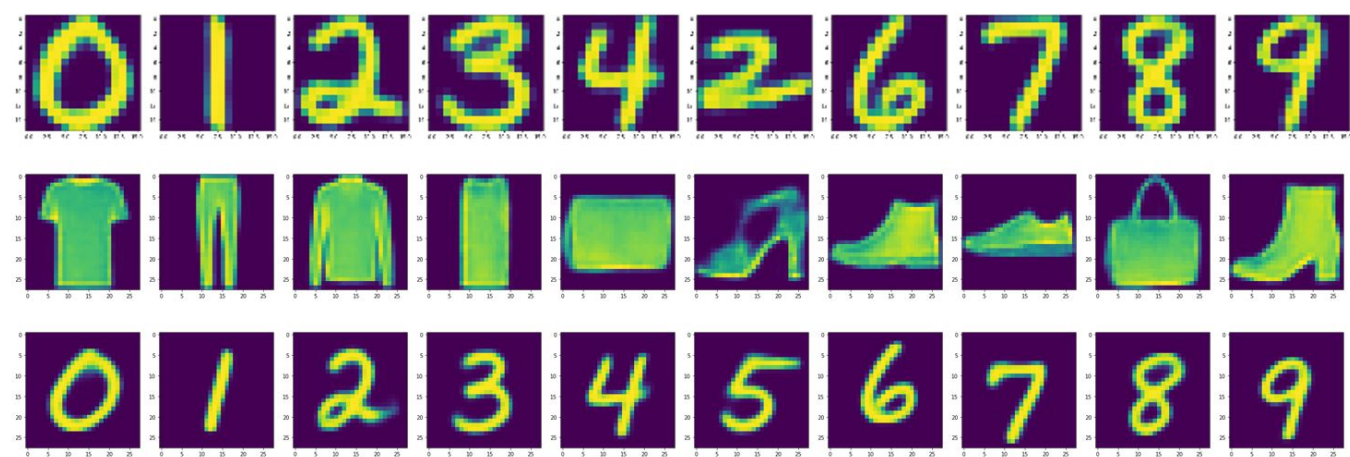

Figure 3.4: Visualizing the reconstruction of clustering centres on 3 different datasets. Top: USPS; Middle: MNIST-FASHION; Bottom: MNIST.

datasets for the DCAE-Kmeans method. This procedure allows for the evaluation of not only the reconstruction quality but also the clustering quality, as the results of our clustering methods can be visually justified and the reasons behind such results can be identified. In the MNIST dataset, the typical reconstruction quality of cluster centres is clearly shown, allowing to visually evaluate the clustering quality. A cluster centre represents the common homogeneous patterns in the latent space for a particular digit. Fig. 3.4 allows for the identification of where the misclustering has occurred. For instance, with the USPS dataset, the model fails to reconstruct the cluster centre of digit 5 and reconstruct a cluster centre similar to digit 2 , indicating that the model has an issue with the samples of digit 5 and misclassifies them as digit 2. Moreover, in the MNIST-Fashion dataset, the model shows the ability to reconstruct the majority of the data cluster centres, however, it fails to reconstruct the cluster centre of the Coat class and Shirt class, allowing us to justify the obtained low clustering results and help us to identify where the clustering error has occurred.

In addition, we carried out a visual assessment where the t-Distributed Stochastic Neighbor Embedding (t-SNE) visualization method [267] was applied to evaluate the clustering results of the proposed methods. The t-SNE is used to visualize high-dimensional data, where it gives each datapoint a location in a two-dimensional map. Fig. 3.5 shows a 2D projection of latent representations obtained with the proposed methods, where the clustering results are colorcoded using ground truth labels. It shows that with joint clustering loss, the learned latent representation space has more compact structures forming significant clusters which match better with the true labels. This is particularly the case with joint clustering loss (Fig. 3.5C. and Fig. 3.5D.), where the learned features have larger inter-cluster distances and tighter structures (see clusters labeled with orange, magenta, pink and dark green colors) compared to the 


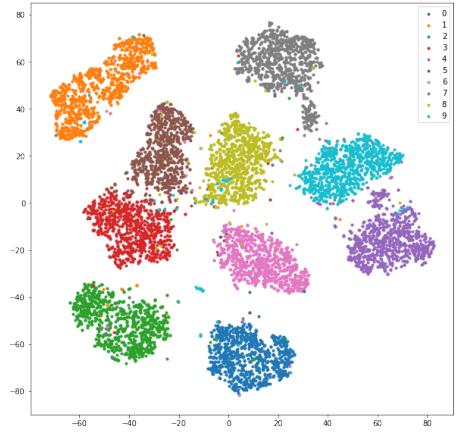

A. DCAE-Kmeans (True Labels)

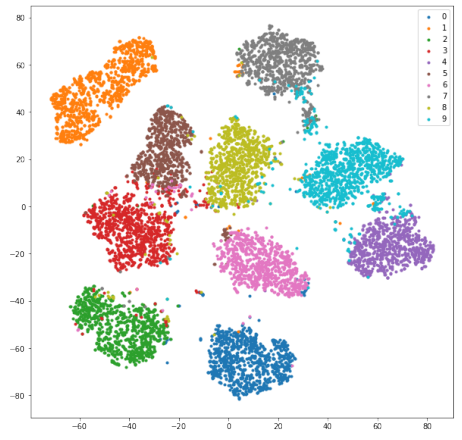

C. DCAE-Kmeans (Our results)

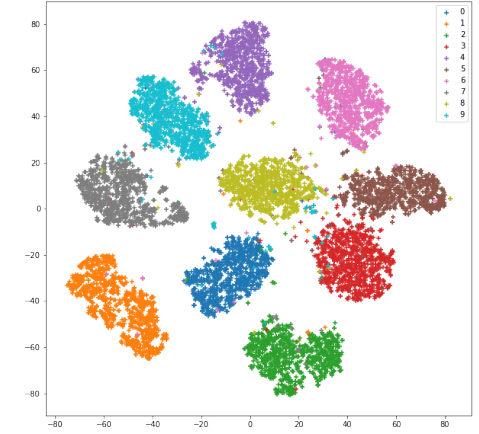

B. DCAE-GMM (True Labels)

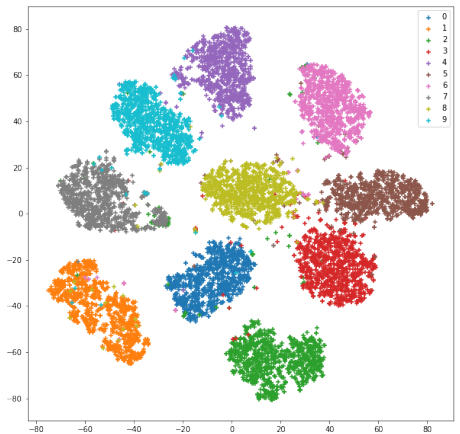

D. DCAE-GMM (Our results)

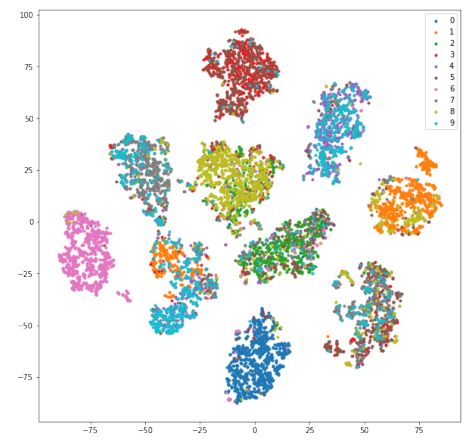

E. DCAE without clustering

Figure 3.5: Visualizations of latent representations in a two-dimensional space with t-SNE on MNIST.

method using no clustering constrains (Fig. 3.5E.). The learned features with no clustering constraint are sparse and not compacted, where lots of outliers can be recognized, and the clusters overlap with each other. Imposing a clustering objective function aids to ensure the newly obtained data representations in the internal layer are assigned to their identical cluster. Although DCAE-Kmeans Fig. 3.5C. and DCAE-GMM Fig. 3.5D. enforce compact representation on a 


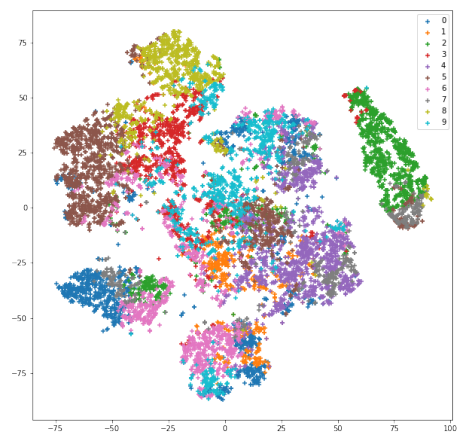

A. Epoch 0

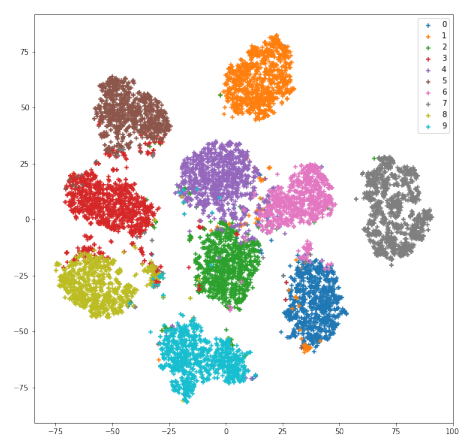

D. Epoch 50

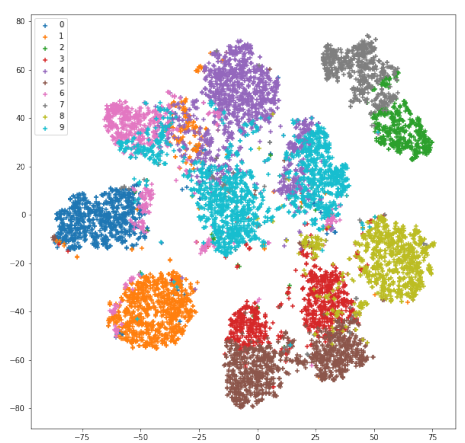

B. Epoch 10

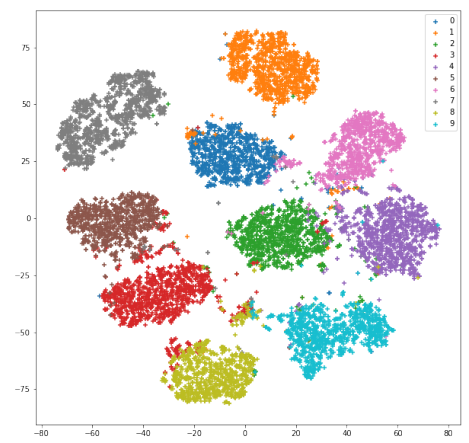

E. Epoch 100

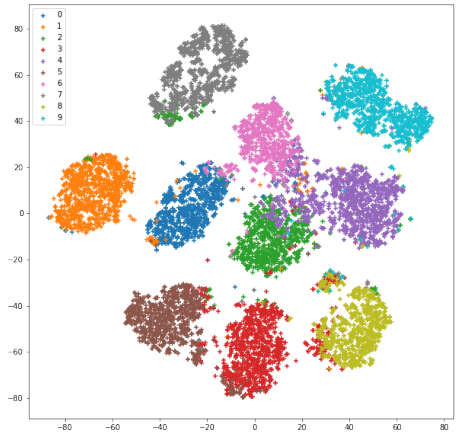

C. Epoch 30

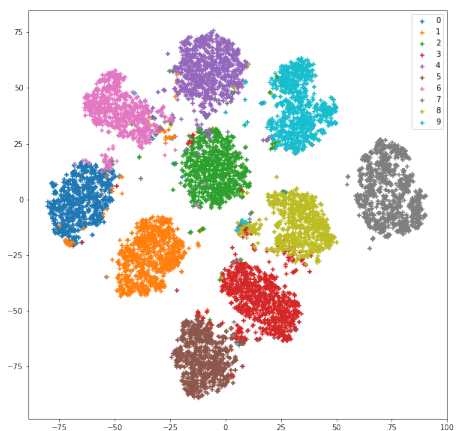

F. Epoch 200

Figure 3.6: Visualizations of latent representations with our clustering results in a two-dimensional space with t-SNE through an iterative training scheme on MNIST.

hidden layer, showing that our proposed objective functions effectively enhance the clustering process and form a kind of structure to increase data compactness, DCAE-GMM can perform much better than DCAE-Kmeans. In the representation of digits 7, 4, and 9 (clusters labeled with the color grey, light blue, and purple), the DCAE-Kmeans model failed to identify some outlier samples, while DCAE-GMM classified them in a much better way, looking qualitatively identical to the ground truth Fig. 3.5B..

Lastly, we applied t-SNE visualization to show the distribution of the latent representations in a two-dimensional space. The learned representations of the MNIST test dataset at different epochs are shown in Fig. 3.6. The figure compares the cluster formation during different epochs of the learning procedure of the DCAE with embedded clustering. At the initial epoch, no clustering structure is observed, and the learned features with DCAE are not discriminative and sparse for clustering (Fig. 3.6A.). After ten epochs, some clusters are initially composed, but the majority of them overlap one another, and clustering outliers are widely ob- 
served (Fig. 3.6B.). For instance, the representation of digits 8, 0, and 1 (clusters labeled with the colors lime, dark blue and orange) begin to form compact clusters, while the representation of digits 6,4 , and 9 (clusters labeled with the colors pink, purple, and light blue) overlap one another. This also occurred with digits 3 and 5 (clusters labeled with the colors red, and brown). It is noteworthy that due to such smoothness the digit 4 has a similar structure to digits 6 and 9 . One reasonable explanation is that all of these digits have a similar structure and the model not trained enough to distinguish them. However, with more training iterations, representative features and useful information are obtained, and similar patterns of the learned representation are locally compacted. Fig. 3.6C. shows that the representations of digits 3 and 5 are distributed and form separate clusters. Similarly, the representations of digits 6, 4, and 9 are far away from each other and form separate clusters. Fig. 3.6D. shows the cluster starting to form the best partitioning of data and maximize the purity of clusters. As the learning scheme continues, the overlapping clusters become discriminative and enforce compact representation, and the intra-cluster variances are reduced significantly, while the inter-cluster distances are enlarged. Furthermore, fewer cluster outliers are observed. The observation is consistent with the results shown in Fig. 3.2.

\subsection{Summary}

In this chapter, we have proposed clustering approaches embedded in a DCAE. The proposed methods alternately learn feature representation and cluster assignment through a DCAE. Both methods consist of clustering and reconstruction objective functions. These procedures enable the classification of a data point into its identical cluster in the latent space in a joint-cost function by alternately optimizing the clustering objective and the DCAE objective, thereby producing stable representations appropriate for the clustering process. The proposed method is trained in an end-to-end way using fixed settings without any pre-training or fine-tuning procedures, enabling a faster training process. We demonstrate the effectiveness of our clustering methods by comparing ours with seven baseline methods on three different image datasets. The experimental results show that DCAE-GMM substantially outperforms all other deep clustering methods. The DCAE-GMM has also performed much better than DCAE-Kmeans, as the DCAE-Kmeans method failed to identify some outlier samples, while DCAE-GMM classified them in a much better way, looking qualitatively identical to the ground truth with visual analysis. The visual assessments demonstrate that as the learning scheme continues, the overlapping clusters become discriminative and enforce compact representation, and the inter-cluster dis- 
tances are enlarged, which indicates that clustering stably converges using an iterative training scheme.

The work on deep clustering contains novel materials, and it has become crucial to explore and extend this with further analysis. The discriminative patterns of the DeepCluster are only discovered through certain parts or objects in an image in an unsupervised manner, where the information necessary to be able to further distinguish between clusters (e.g. supervision information which aids the learning of discriminative feature representations) is not available. To address this, the following chapter investigates ways to reinforce the performance of deep clustering methods and provides an analytical study for understanding the effectiveness of differing discriminatory power, focusing on strengthening and discriminating the learned features through deep clustering methods. The discriminative feature representation is then utilized in Chapter 5 and 6 to measure the importance of a network's units for the purpose of network compression. Finally, we apply what we proposed in this chapter to real-world data; a case study in which the DeepCluster method is used to cluster animal behaviors through movement is presented in Chapter 7. 


\section{Chapter 4}

\section{Learning Discriminatory Deep Clustering Models}

\section{Contents}

4.1 Introduction $\ldots \ldots \ldots \ldots \ldots \ldots \ldots \ldots \ldots$

4.2 Proposed Methods . . . . . . . . . . . . . . . . 78

4.2.1 DeepCluster: A DCAE with Embedded Clustering . . . . . . . 79

$4.2 .2 \quad$ Architecture . . . . . . . . . . . . . . . . . . . . . . . . 79

4.2.3 Graph-Based Activity Regularization (GBAR) . . . . . . . . 80

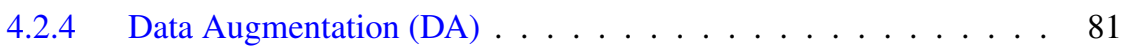

4.2.5 Extended Output Layer and Different Levels of Supervision _ . . 82

4.3 Experimental Results . . . . . . . . . . . . . . . . . . 83

4.3.1 Regularizations for DeepCluster . . . . . . . . . . . . . 84

4.3.2 Learning Discriminatory Deep Clustering Models Through Different Levels of supervision . . . . . . . . . . . . . 87

4.3.3 Deep Clustering Through Various Levels of Supervision _. . . . 91

4.4 Summary . . . . . . . . . . . . . . . . . . . 93 


\subsection{Introduction}

In recent years, different approaches to unsupervised deep clustering have been developed utilizing deep neural networks [20]. A deep convolutional auto-encoder (DCAE) has been exploited for clustering analysis, allowing clustering algorithms to deal with abstract latent representations in a low-dimensional space. DCAE is a deep unsupervised model for representation learning. It maps inputs into a new latent space, allowing for the acquisition of useful feature representations via its encoding layer. These high-level representations provide beneficial properties that support traditional clustering algorithms in demonstrating a satisfying performance.

DeepCluster [5], a DCAE with embedded clustering which is proposed in the previous chapter, is a deep unsupervised clustering method that simultaneously captures representative features and the relationships among images, extracts similar patterns in new space representations, and finds ideal representative centers for distributed data. In this procedure, the discriminative patterns are only discovered through certain parts or objects in an image in an unsupervised manner. The goal of this method is to learn feature representations and cluster assignments simultaneously, utilizing the strength of the DCAE to learn high-level features in the latent space. Two objective functions were utilized: one was embedded into a DCAE model to minimize the distance between features and their corresponding cluster centres, while the other was used to minimize the reconstruction error of the DCAE. During optimization, all data representations are assigned to their new identical cluster centres, after which the cluster centres are updated iteratively, allowing the model to achieve a stable clustering performance. The defined clustering objective, as well as the reconstruction objective, are simultaneously utilized to update the parameters of the transforming network.

In an attempt to investigate the ways in which to reinforce the performance of conventional clustering methods, several methods have been developed to study semi-supervised clustering approaches [191-196], which aim to cluster a large amount of unlabeled data in the presence of minimal supervision. Similarly, several supervised clustering methods [198-202] have been proposed. Their core idea is to include a supervisory scheme into the clustering process, so as to improve unsupervised clustering performance by exploiting supervised information. Conventional supervised and semi-supervised clustering approaches have received a lot of attention; however, despite the substantial success of deep learning, limited attention has been paid to deep supervised and semi-supervised clustering methods. Therefore, providing a way to inject varying degrees of supervision into the body of the learning process and exploring the influence 
of adding supervision knowledge into a deep clustering method are worthwhile endeavors in order to distinguish the discriminative powers obtained by patterns in an unsupervised manner or provided by supervision components. To empirically analyze the above insight, a new version of DeepCluste is introduced to involve a supervision component and assist with forming a kind of a structure that reconciles discriminative features discovered by the clustering process and discriminative features provided by labeling patterns. This mechanism ensures that the learned features derived from the encoding layer are the best discriminative attributes. In other words, it looks to find a well-defined structure for clusters, through the encoding part of the DCAE, in the presence of discriminative power attributes.

Furthermore, most of the previously mentioned methods focus on providing supervision to the clustering process; however, their focus was on modifying the clustering methods by imposing more constraints. Limited attention was paid to strengthening the discriminative features, which would facilitate the job of traditional clustering algorithms. Therefore, taking advantage of deep learning methods to strengthen the discriminatory power of the learned features through the learning process is deserving of study, where the deep clustering algorithm can be supported in demonstrating satisfying performance. Our key insight is that a DCAE with embedded clustering can have robust discriminative power, as the learned features are highly expressive and can be worthy injective functions. As a result, we introduce a deep clustering method that is carried out in the presence of varying degrees of discriminative power. To the best of our knowledge, our work provides the first analytical study for understanding the effectiveness of the discriminatory power obtained by the use of two discriminative attributes: data-driven discriminative attributes with the support of regularization techniques, and supervision discriminative attributes with the support of the supervision components. Although the data augmentation technique has received considerable attention in supervised learning as a way to tackle the generalization issue, it has been overlooked by most of the deep clustering methods. To fill this gap, we introduce the data augmentation technique into the method of DeepCluster and study the effectiveness of the discriminatory power obtained by data augmentation patterns in deep clustering performance.

In this chapter, we focus on deep clustering methods in which varying degrees of discriminative powers can be imposed on the clustering layer or injected into the body of the learning process. We propose a new version of the DeepCluster to allow for the imposing of regularization techniques and the involvement of a supervision component. This mechanism allows us to experience different discriminatory powers and examine the clustering performance through 
different discriminative attributes. We consider two regularization techniques: one that is embedded into the clustering layer, and another that is used during the training process. We also consider three different learning levels: supervised, semi-supervised and unsupervised. We evaluate our experimental methods on MNIST, USPS, MNIST fashion, and SVHN datasets. Furthermore, we show the deep clustering performance at certain levels of supervision as well as the impact of the regularization techniques.

The rest of the chapter is organized as follows: in section 4.2, we present our methodologies and approaches. In section 4.3, we present our experimental results, and finally, concluding remarks are given in section 4.4.

\subsection{Proposed Methods}

The proposed approach is a deep clustering method that is carried out in the presence of varying degrees of discriminative power. It introduces a mechanism with which various levels of supervision are injected into the body of the learning process. This mechanism allows us to explore the impact of supervised information on the achievement of our deep clustering method. The effectiveness of the discriminatory power that is obtained by discriminative attributes with the support of regularization techniques is also studied. It allows us to investigate the discriminative power resulting from the inclusion of regularization and data augmentation techniques into the body of the deep clustering model.

We consider two regularization techniques and three different learning levels. A discriminative representation, via the internal layer of DCAE, is exploited to support the clustering task in the presence of varying degrees of supervision, regularization and data augmentation techniques. A combination of methods is imposed on the DCAE architecture or injected into the body of the learning process to strengthen the learned features and support the performance of the deep clustering. Each experimental model consists of a combination of objective functions, some of which minimize the clustering loss, the reconstruction loss, and the categorical crossentropy function of the provided supervision. All objectives are simultaneously optimized.

The following subsections clarify our methodology. First, we briefly review how the DeepCluster works and define the necessary loss functions when introducing the proposed methods. After that, we describe the architecture used in our methods. Then, we explain the regularization and data augmentation techniques and how they are utilized to support the learning of discriminative features. Finally, we introduce a new version of the DeepCluster, where the out- 
put layer is extended to allow for the injection of different levels of supervision into the body of the learning process.

\subsubsection{DeepCluster: A DCAE with Embedded Clustering}

In the encoding part of DCAE, convolutional layers are used as feature extractors to learn features through mapping the data into an internal layer. Then, the deconvolutional layers are used to reconstruct the data representation to its original shape by minimizing the reconstruction error using the Euclidean (L2) loss function.

$$
E_{1}=\frac{1}{N} \sum_{n=1}^{N}\left\|x_{n}-\hat{x}_{n}\right\|^{2}
$$

where $\hat{x}$ is a reconstructed image, and $x$ is an original image.

Although DCAE learns effective representations via its encoding layer, it does not explicitly force representation to form compact clustering. In the previous chapter, we proposed the DeepCluster that learns feature representations and clusters assignments simultaneously. It embeds a clustering objective function into a DCAE framework that minimizes the distance between data points and their assigned centers in the latent space as follows:

$$
E_{2}=\lambda \cdot \frac{1}{N} \sum_{n=1}^{N}\left\|h^{t}\left(x_{n}\right)-c_{n}^{*}\right\|^{2}
$$

where $\lambda$ is a weight-parameter that controls the contribution percentage of a certain cost function in the overall cost function, $N$ is the number of data examples, $h^{t}(*)$ denotes the encoded representation obtained at the $t^{\text {th }}$ iteration, $\left(x_{n}\right)$ is the $n^{\text {th }}$ example in the dataset $x$, and $c_{n}^{*}$ is the assigned centroids to the $n^{\text {th }}$ example. At each iteration, the model optimizes two components using an optimizer and backpropagation: (1) network parameters as well as a mapping function $h$, and (2) cluster centers $c$. After each internal representation is assigned to the closest cluster center, the cluster centers are iteratively updated.

\subsubsection{Architecture}

Our DCAE architecture consists of three convolutional layers. This is followed by two fullyconnected layers, of which the second layer (clustering layer) has ten neurons. These are considered to be hidden representations learned through the training process. A single fullyconnected layer is followed by three deconvolutional layers as the decoding part, and ReLU is utilized as the activation function. Table 4.1 shows a detailed configuration of the DCAE 
Table 4.1: Detailed configuration of the DCAE network architecture used in the experiments.

\begin{tabular}{|c|c|c|c|c|}
\hline Layer & MNIST & USPS & MNIST Fashion & SVHN \\
\hline Convolutional & $5 \times 5 \times 32$ & $4 \times 4 \times 32$ & $5 \times 5 \times 32$ & $5 \times 5 \times 32$ \\
\hline Convolutional & $5 \times 5 \times 64$ & $4 \times 4 \times 64$ & $5 \times 5 \times 64$ & $5 \times 5 \times 64$ \\
\hline Convolutional & $3 \times 3 \times 128$ & $2 \times 2 \times 128$ & $3 \times 3 \times 128$ & $2 \times 2 \times 128$ \\
\hline Fully-Connected & 1152 & 512 & 1152 & 2048 \\
\hline Fully-Connected & 10 & 10 & 10 & 10 \\
\hline Fully-Connected & 1152 & 512 & 1152 & 2048 \\
\hline Deconvolutional & $3 \times 3 \times 128$ & $2 \times 2 \times 128$ & $3 \times 3 \times 128$ & $2 \times 2 \times 128$ \\
\hline Deconvolutional & $5 \times 5 \times 64$ & $3 \times 3 \times 64$ & $5 \times 5 \times 64$ & $5 \times 5 \times 64$ \\
\hline Deconvolutional & $5 \times 5 \times 32$ & $3 \times 3 \times 32$ & $5 \times 5 \times 32$ & $5 \times 5 \times 32$ \\
\hline
\end{tabular}

network architecture for all experimental datasets. Our extensions to this architecture are as follows: firstly, the learned features given by the encoding layer are optimized to form compact and discriminative clusters using a clustering objective function, which minimizes the distance between a feature representation and its respective centroid. Secondly, at the same time as minimizing the reconstruction loss, we iteratively optimize the mapping function of the encoding part and cluster centres to obtain more effective clustering. Thirdly, we modify the architecture to include the regularization terms; see section 4.2.3 for details. Lastly, instead of a reconstruction layer at the end of the DCAE, extra layers are added at the end of the network just after the reconstruction layer to inject different degrees of supervision; see section 4.2.5 for details.

\subsubsection{Graph-Based Activity Regularization (GBAR)}

A combination of activity regularization techniques, which were recently proposed in [268], are included in the clustering layer. This allows us to examine the achievements of the deep clustering method with regularizer terms. The activity regularization techniques ensure each node is specialized and enforce inputs to equally spread out over the clustering layer nodes. We adopt these techniques in combination with our clustering loss to determine the impact of the regularization techniques on the clustering performance

Considering $B$ represents the outputs of the clustering layer for all $m$ examples with $n$ number of output nodes, $N$ is the symmetric matrix defined as $N=B^{T} B$. In order to obtain an identity matrix, affinity enforces the off-diagonal entries to be zeros, which ensures that a single 
input can only have one high activation node, allowing for the specialization of the nodes. The input must belong to one of the clusters. Otherwise, it will cause some errors in clustering.

$$
\text { Affinity }=\alpha(B)=\frac{\sum_{i \neq j}^{n} N_{i j}}{(n-1) \sum_{i=j}^{n} N_{i j}} .
$$

The diagonal entries of $N$ is a 1-D vector called $v . V$ is a symmetric matrix that results from the manipulation of the transpose of the diagonal entries of $N$ by the diagonal entries, defined as $V=v^{T} v$. The term (Balance) forces inputs to equally spread out over the clustering layer nodes, ensuring all nodes in the clustering layer are equally activated within one batch.

$$
\text { Balance }=\beta(B)=\frac{\sum_{i \neq j}^{n} V_{i j}}{(n-1) \sum_{i=j}^{n} V_{i j}} .
$$

The overall cost function is a combination of loss functions and regularization terms: the first part essentially minimizes the reconstruction objective function $E 1$, by Eq.(4.1), and clustering objective function $E 2$, by Eq.(4.2). The rest of the overall cost function is regularization terms.

$$
\min _{W, b} E_{1}+E_{2}+c_{\alpha} \alpha(B)+c_{\beta}(1-\beta(B))+c_{F}\|B\|_{F}^{2},
$$

where $\alpha($.$) and \beta($.$) are the unsupervised regularization terms respectively defined in Eq.(4.3),$ Eq.(4.4), $\|B\|_{F}$ denotes to the Frobenius norm for $B$, and $c_{\alpha}, c_{\beta}, c_{F}$ are the regularization weighing coefficients.

\subsubsection{Data Augmentation (DA)}

Data augmentation is a regularization technique widely used in supervised deep learning models to improve their generalization. Data augmentation is the process of generating transformed versions of original images in the training dataset. Transforms include a range of operations such as random rotation, flipping, shifting, zooming and cropping, and much more. The local structure preservation distinguishes the convolutional neural network (CNN), so it learns features that are invariant to their local information in the image. Data augmentation can aid a model to learn features that are invariant to transformation and can support learning using the transform invariant approach. Therefore, it is worthwhile to study the effectiveness of the discriminatory power obtained by data augmentation patterns in an unsupervised clustering method. To that end, we introduce a data augmentation technique into the DeepCluster. 
Given a set of training samples $X_{n}$, where $n$ donates the number of samples. For each example $X_{i}$, we can apply augmentation using the following form: $\hat{X}_{i}=T_{\text {augmentation }}\left(X_{i}\right)$. In the DCAE, the reconstruction objective function utilized in this case is:

$$
E_{1}=\frac{1}{N} \sum_{n=1}^{N}\left\|\hat{x_{n}}-\hat{y_{n}}\right\|^{2}
$$

where $\hat{x}$ is an augmented version of the original image $x$, and $\hat{y}$ is a reconstructed image of the augmented version.

\subsubsection{Extended Output Layer and Different Levels of Supervision}

We utilize the network architecture, discussed in section 4.2.2. Our extension to the architecture is that instead of a reconstruction layer at the end of the DCAE, an extra layer is added at the end of the network, just after the reconstruction layer. This allows us to inject supervision knowledge across the learning process and also examine clustering performance with different discriminatory power provided by supervision knowledge. The new version of the DeepCluster method allows us to utilize its strength to obtain discriminative and robust features from the encoding layer. It also allows the deep clustering method to extract more discriminative features and cluster assignments simultaneously.

We use the same architectures in the supervised and semi-supervised models, as shown on Table 4.1. Instead of a reconstruction layer at the end of the DCAE, we flatten the output of the reconstruction layer and feed it into a certain number of nodes in the last layer. The number of nodes depends on the task at hand, i.e. the number of provided classes (e.g. ten nodes for the supervised case and two nodes for the semi-supervised case). A softmax function is used for the final prediction. The final architecture of our extended version of the DeepCluster is presented in Fig. 4.1.

Two forms of labels are used, true labels and parent-class labels, to reflect two different levels of supervision. True labels are provided in the supervised training process. Parent-class labels are used in semi-supervised deep clustering, where true class labels are combined to form parent-class labels. For example, in the clustering digit images created using the the proposed DCAE, the parent-class labels are defined as:

$$
\text { ParentLabel }=\left\{\begin{array}{ll}
0 & \text { Labels }<5 \\
1 & \text { otherwise }
\end{array} .\right.
$$




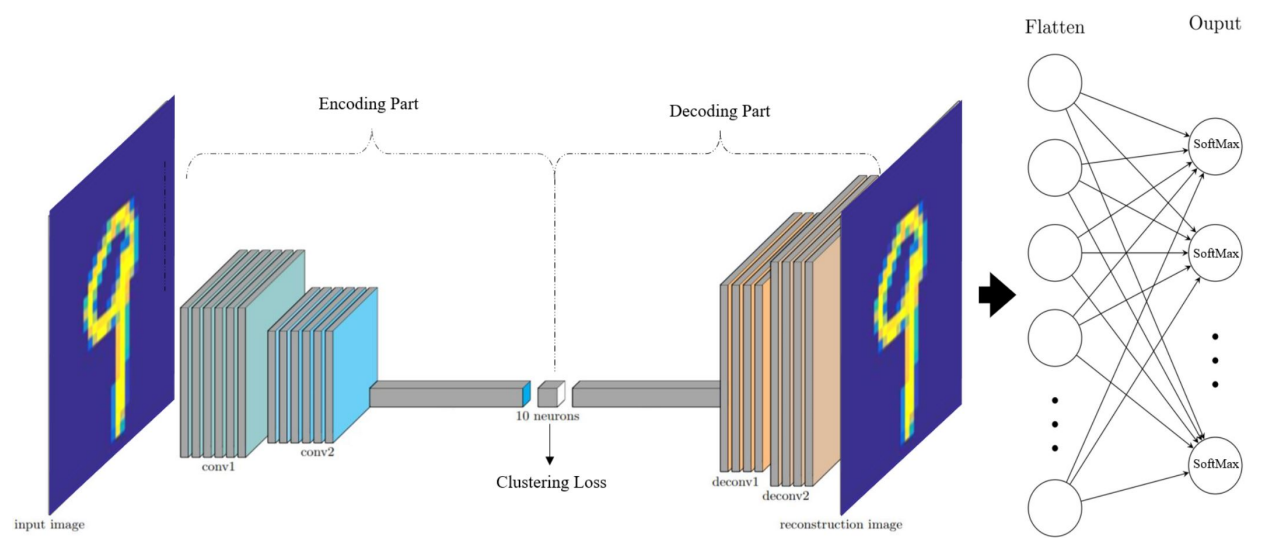

Figure 4.1: The architecture of the discriminatory DeepCluster models. An extra layer is added at the end of the network just after the reconstruction layer to inject different degrees of supervision.

The categorical cross-entropy function between network predictions and provided labels is defined as:

$$
E_{3}=-\sum_{j} t_{i, j} \log \left(p_{i, j}\right)
$$

where $p$ is prediction, $t$ is the sample label, $i$ indexes samples, and $j$ indexes classes. In the DCAE hidden layer, encoded features are used to compute clustering loss function in order to minimize the distance between data points and their corresponding cluster centers.

The overall cost function is thus a combination of reconstruction loss $E 1$, by Eq.(4.1), clustering residual in latent space $E 2$, by Eq.(4.2), and categorical cross-entropy loss $E 3$, by Eq.(4.8) that minimizes the classification error with any supervised or semi-supervised scheme:

$$
\min _{W, b} E_{1}+E_{2}+E_{3}
$$

\subsection{Experimental Results}

Learning discriminatory deep clustering models through adopting a variety of regularization techniques or through injecting various levels of supervision into the learning process is a unique mechanism. It helps to reconcile extracted latent representations effectively and provide discriminative power to produce the best discriminative attributes. The key idea of our approach is to distinguish the discriminatory power of numerous formats (e.g. through adopting GBAR and DA techniques or through varying degrees of supervision) when searching for a 
Table 4.2: Details of the datasets used in our experiments for the discriminatory DeepCluster models.

\begin{tabular}{|r|c|c|c|c|}
\hline Dataset & Examples & Classes & Image Size & Channels \\
\hline MNIST [83] & 70000 & 10 & $28 \times 28$ & 1 \\
\hline USPS [260] & 11000 & 10 & $16 \times 16$ & 1 \\
\hline MNIST Fashion [261] & 70000 & 10 & $28 \times 28$ & 1 \\
\hline SVHN [270] & 99289 & 10 & $32 \times 32$ & 3 \\
\hline
\end{tabular}

compact structure with which to form robust clusters. We first studied the achievements of our DeepCluster method when adopting GBAR and DA techniques. After this, we analyzed the impact of injecting different levels of supervision into a deep clustering method. Lastly, we investigated the deep clustering performance when adopting the GBAR regularization technique with various levels of supervision.

\section{Implementation Details}

The proposed methods were implemented using Keras [265] and Theano [269] in Python and evaluated on four different datasets; MNIST [83], USPS [260], MNIST fashion [261], and SVHN [270] datasets. Some of these are the most commonly used datasets in previous literature published in the field of deep clustering methods. The specifications of these datasets are presented in Table 4.2. The proposed models were trained end-to-end without involving any pre-training or fine-tuning procedures. All weights and cluster centers were initialized randomly. The Adam [63] optimizer was used, where each batch contains 100 randomly shuffled images. We set $\lambda$, which is the clustering weight-parameter that controls the loss contribution percentage of clustering error, to 0.1 . Throughout our experiment, we used a fixed learning rate of 0.006 , a momentum of 0.9 , and a weight decay of 0.0005 .

\subsubsection{Regularizations for DeepCluster}

Here, we study the impact of embedding a variety of regularization techniques (e.g. GBAR or DA) into a deep clustering method. This procedure allows us to analyze the discriminative powers of the learned features by adopting GBAR or DA and examine the achievements of our DeepCluster method. We use a DCAE model to map the data, via a series of convolutional layers, into latent representation, and embed the Kmeans clustering algorithm as well as regularization techniques into an integral deep clustering framework. This process enables 

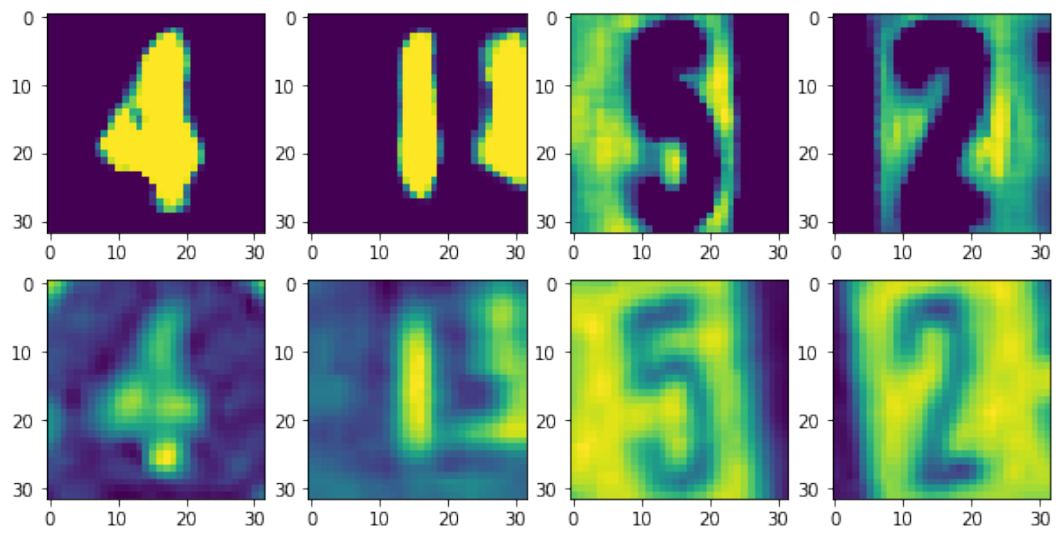

Figure 4.2: Visualizations of reconstruction images (Top) and input images (Bottom) in SVHN dataset.

Table 4.3: Comparison of clustering quality of the DeepCluster method using accuracy evaluation metric with and without regularizations.

\begin{tabular}{|r|c|c|c|c|}
\hline Regularization & MNIST & USPS & MNIST fashion & SVHN \\
\hline None & $93.42 \%$ & $83.25 \%$ & $58.20 \%$ & $17.41 \%$ \\
\hline GBAR & $93.37 \%$ & $73.49 \%$ & $57.79 \%$ & $17.05 \%$ \\
\hline DA & $\mathbf{9 5 . 4 6 \%}$ & $\mathbf{8 4 . 8 1 \%}$ & $\mathbf{6 0 . 0 6 \%}$ & $\mathbf{2 2 . 3 0 \%}$ \\
\hline
\end{tabular}

the deep clustering method to extract more enhanced discriminative features while clustering assignments in a simultaneous manner. The deconvolutional layers are, thereafter, used to reconstruct the data representation to its original shape.

The GBAR regularization technique, discussed in section 4.2.3, is embedded into the clustering layer, which is the last layer of the encoding part of the DCAE. Said technique ensures that each node in the clustering layer is specialized and forces inputs to spread out over the clustering layer nodes equally. Considering $B$ is the output of the clustering layer, which forms an $m \times 10$ matrix, where $m$ is the number of examples and 10 is the output nodes of the encoding part, which represent the number of clusters in our experiments. $N$ is $10 \times 10$ symmetric matrix defined as $N=B^{T} B$, which is enforced to be an identity matrix, called affinity regularization. This term ensures a single input can have only one high-activation node, allowing specialization of such a node, while the term of balance regularization enforces inputs to equally spread out over the clustering layer nodes, which ensure all nodes in the clustering layer are equally activated within one batch. The DA, on the other hand, is used as a regularization to help a model avoid the over-fitting issue and learn invariant features to support the performance of 
the DeepCluster method. The utilized DA comprises a suite of techniques, including various degrees of flipping and rotation, which can enhance more discriminative power, such that better deep clustering can be achieved using them.

To validate the effectiveness of consolidating regularization techniques, we compare the clustering performance of our DeepCluster method with and without regularization techniques. Table 4.3 compares the results of the unsupervised deep clustering methods using the reconstructed loss. It shows that the adoption of the GBAR technique did not significantly affect the result of clustering accuracy, neither did it add any discriminative ability to the deep clustering method. Essentially, at the beginning of learning, the clusters have not yet formed, and thus, activation of one node in a layer from an input does not reflect the actual situation and thus multiple nodes should be allowed to be activated. In contrast, the adoption of the DA technique resulted in moderate improvement in clustering accuracy. In other words, the accuracy of the DeepCluster method on MNIST, USPS, and MNIST Fashion increased by $2.04 \%$, $1.56 \%$, and $1.86 \%$ respectively, compared to the method which did not use any regularization technique. The improvement is still observable even on the more difficult dataset SVHN. Our unsupervised deep clustering method did not perform well for the SVHN dataset, even though it retrieved the effective latent representation into an appropriate approximation of the original input data Fig. 4.2. The reason is that instead of clustering the digits' patterns, the clustering method intends to group the data examples based on their backgrounds' shape and color.

Table 4.3 demonstrates that the adoption of the DA can improve the clustering accuracy, which is consistent with a recent study by [266]. Our result proves that the adoption of the DA can moderately provide a discriminative power to the DeepCluster method. One reasonable explanation for this minor improvement is the invariance of the learned features of the unsupervised deep clustering method. In contrast to supervised learning, the learned features of unsupervised learning are more invariant, and the aid of generating transformed versions of original images can be limited to improve the clustering performance significantly. This fact motivated us to investigate and analyze the invariance properties of the learned representation in the latent space through different levels of supervision, see section 4.3.2 for details.

The utilization of the GBAR technique is intended to enforce the clustering process and impose restrictions when clustering the feature representations of the DCAE. Our results demonstrate that obtaining clustering-friendly latent representations through unsupervised DCAE model, where the learned features are invariant, could be sufficient to allow our DeepCluster method to yield a substantially better performance for both reconstruction and clustering qual- 
ity without any enforcement. Therefore, adopting a regularization technique such as GBAR is useless in this case as it may depend on when such regularizations are imposed. Given that a DCAE offers clustering-friendly discriminative latent representations by minimizing the reconstruction error, the learned features can be efficiently clustered without any regularization technique. The GBAR technique [271] has shown its ability to help clustering latent representation when the hidden information is captured through the help of discriminative models (e.g. semi-supervised process [272] or an initialized supervised pertaining process [268]). These findings motivated us to investigate and analyze the impact of adopting GBAR into a deep clustering method when injecting different levels of supervision, see section 4.3.3, for details.

\subsubsection{Learning Discriminatory Deep Clustering Models Through Different Levels of supervision}

Here, we extend the architecture of the DCAE and add an output layer to inject supervision information into the body of the learning process. In the encoding part of the DCAE, an abstract latent representation is learned in a discriminative way to capture not only the discriminative attributes from the data, but also patterns provided by supervision components. The injection of the supervision into a DCAE framework provides a powerful discriminative capability that supports our deep clustering method in demonstrating satisfying performance. To thoroughly investigate the abilities of our proposed method, we first evaluated the clustering performance in the presence of minimal supervision. After that, we visually and empirically evaluated the impact of supervision on the deep clustering methods. Then, we evaluated the clustering performance with different supervision schemes. Lastly, we analyzed the invariance properties of learned representation, given different levels of supervision.

\subsubsection{Deep Clustering Performance with Minimal Supervision}

For the MNIST dataset, the experiments were performed using four different numbers of trained examples, i.e. 2000, 4000, 6000, 8000. In other words, we only used a limited number of examples at the training stage to simulate data with a shortage of labeled data. We trained our supervised model using these settings with the same number of iterations. The comparative results are shown in Table 4.4, and support our hypothesis that a small amount of labeled data can add enough discriminative ability to unsupervised deep clustering. Note that the results are the accuracy of clustering, not classification using reconstructed image. 
Table 4.4: Number of samples used in the training stage and clustering accuracy.

\begin{tabular}{|c|c|}
\hline Trained Examples & Clustering Accuracy \\
\hline 2000 & $94.24 \%$ \\
\hline 4000 & $96.48 \%$ \\
\hline 6000 & $97.52 \%$ \\
\hline 8000 & $98.06 \%$ \\
\hline
\end{tabular}

\subsubsection{Visualizing Learned Representation Through Different Levels of Supervision}

In order to visualize the impact of supervision in deep clustering, the t-SNE visualization method [267] was applied as a visual assessment to show that the addition of supervision allows to guide the clustering task to obtain more appropriate data partitioning and present demarcated clusters relevant to the supervised learning task. Fig. 4.3 shows the latent representation of our proposed methods in $2 \mathrm{D}$ space using different levels of supervision; the color-coding of the ground truth labels are used to visualize the clustering results. This visualization shows that adding a supervision component into the deep clustering method produces significantly more compact clusters, which have a better matching with true labels Fig. 4.3 (top row). The learned features involve a supervised process has tighter structures and larger inter-cluster distances compared with semi-supervised and unsupervised models. Injecting supervision into the learning process effectively reconciles data-driven obtained representations and the provided supervision knowledge to form the best partitioning of data and maximize the purity of clusters. With the semi-supervised model (Fig. 4.3E.), the clustering results show typical compact clusters, producing much better clustering results compared with the unsupervised model (Fig. 4.3, Right), where the learned features are sparse and not compact. Although the visualization of (Fig. 4.3F.) shows compact clusters, when comparing this with (Fig. 4.3C.), it appears the learned features via the unsupervised model do not match the true labels. That is illustrated by the overlapping colors on (Fig. 4.3F.). Compared to (Fig. 4.3F.) which enforces compact representation on a hidden layer, the clusters formed by normal DCAE still present ten distinct clusters, although they have higher intra-cluster variance and lower inter-cluster difference. Thus, by adopting semi-supervised (see Fig. 4.3E.) and supervised models (see Fig. 4.3D.), intra-cluster variances are reduced significantly while the inter-cluster distances are enlarged. In particular, less cluster outliers are observed in Fig. 4.3D.. 


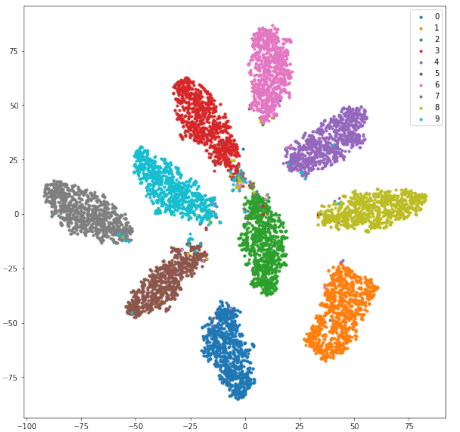

A. Supervised (Labels)

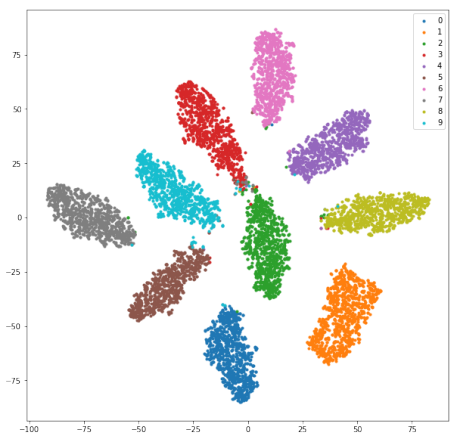

D. Supervised (Our Results)

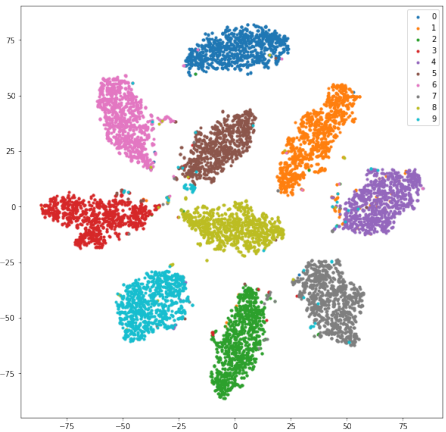

B. Semi-supervised (Labels)

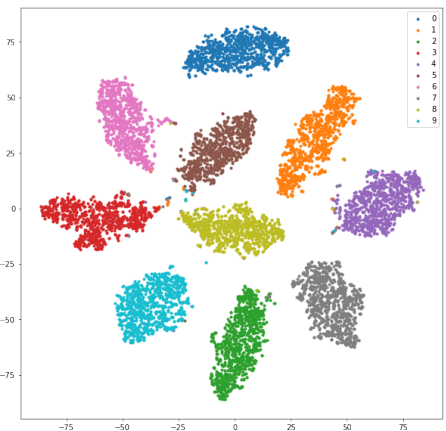

E. Semi-supervised (Our Results)

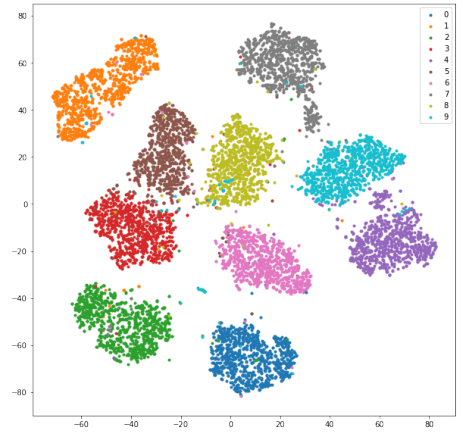

C. Unsupervised (Labels)

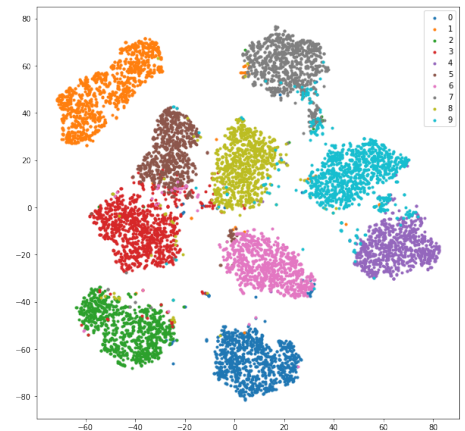

F. Unsupervised (Our Results)

Figure 4.3: Visualizations of latent representation for our deep clustering method through through different levels of supervision on the MNIST testing set. Top: True Labels. Bottom: Our Results

\subsubsection{Deep Clustering Performance Through Different Levels of Supervision}

We empirically evaluated the performance of representation learning of the DCAE with different supervision schemes by comparing the clustering accuracy against the true label. These experiments show that a discriminative representation can form a kind of structure that reconciles the one discovered by the clustering process and the one formed by labeling patterns. An available side of label information along with data-driven patterns are efficiently brought together to support the clustering process. Injecting true labels or partial supervision into the deep clustering method allows the clustering algorithm to perform much better compared with the models that utilize an unsupervised learning process. Label consistency can add a discriminative power that clearly guides the clustering algorithm to obtain the best accurate compacted groups compared with data-driven discriminative attributes from data patterns only. The full supervisory mechanism substantially improves the results of clustering, while the mechanism of partial supervision enhances the clustering algorithm to reveal more accurate compacted 
groups compared to unsupervised deep clustering methods. Table 4.5 summarizes the experimental results on four different datasets including MNIST, USPS, and more challenging ones, such as MNIST fashion and SVHN. Since the performances were evaluated on a clustering task, the accuracy increasing with supervision knowledge can be observed in both cases. Particularly for the SVHN dataset, the accuracy is boosted by more than double when weak labels are provided. We argue that the common structures are not well-formed without supervision, where large appearance variance and image noise are commonly observed in the SVHN dataset.

Table 4.5: Comparison of clustering accuracy on four different datasets using different learning levels.

\begin{tabular}{|r|c|c|c|c|}
\hline & MNIST & USPS & MNIST fashion & SVHN \\
\hline Unsupervised & $93.42 \%$ & $83.25 \%$ & $58.20 \%$ & $17.41 \%$ \\
\hline Semi-supervised & $97.77 \%$ & $91.92 \%$ & $63.59 \%$ & $43.96 \%$ \\
\hline Supervised & $\mathbf{9 8 . 8 2 \%}$ & $\mathbf{9 5 . 0 6 \%}$ & $\mathbf{8 8 . 7 3 \%}$ & $\mathbf{9 2 . 4 0 \%}$ \\
\hline
\end{tabular}

\subsubsection{Invariance Properties of the Learned Representation Through Different Levels of Supervision}

We analyzed the invariance properties of learned representation given different levels of supervision. We trained five different models with varying degrees of supervision: supervised, semi-supervised with three different percentages of supervision (20\% (Semi-1), 30\% (Semi2), 50\% (Semi-3)), and unsupervised. We applied a range of rotation-based transformations (rotate by $90^{\circ}, 180^{\circ}, 270^{\circ}$, flip horizontally, flip horizontally and rotate by $90^{\circ}, 180^{\circ}, 270^{\circ}$ ) to each image. Moreover, we followed [273,274] to measure the variance properties by calculating the Mean Squared Error (MSE) between the features of the original images and the transformed ones. The comparison results of invariance properties of the learned representation in four different layers are shown in Fig. 4.4. This allows for the tracking of the changes of invariance properties through different layers, where the encoding layer (Fig. 4.4D.) is shown to be the most robust and invariant layer. The figure also compares the invariance properties of learned representation in five different models. Overall, the experiment empirically confirms that the features are more invariant when no supervision is provided. In other words, the features learned by the unsupervised model are more invariant compared to features learned with supervision. With the semi-supervised approach, the invariance properties of the learned representation differ based on the provided percentages of supervision. This indicates that the 
more limited a supervision percentage is, the more invariant and stable the obtained learned representations are. However, such invariant representations are not expressive and thus lead to worse clustering results.

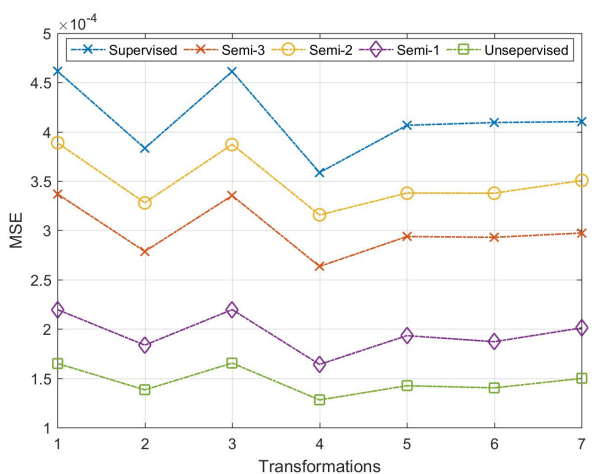

A. Conv-1

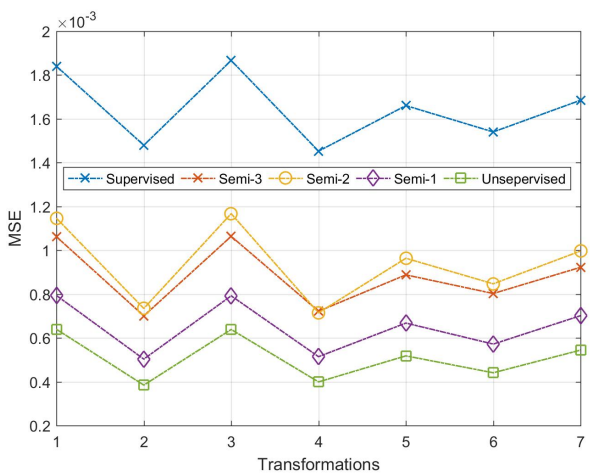

C. Conv-3

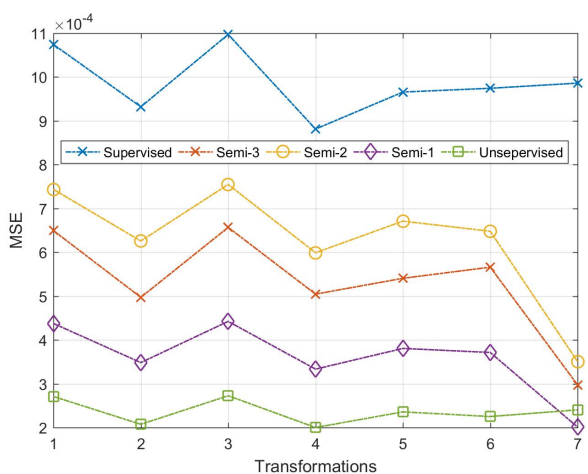

B. Conv-2

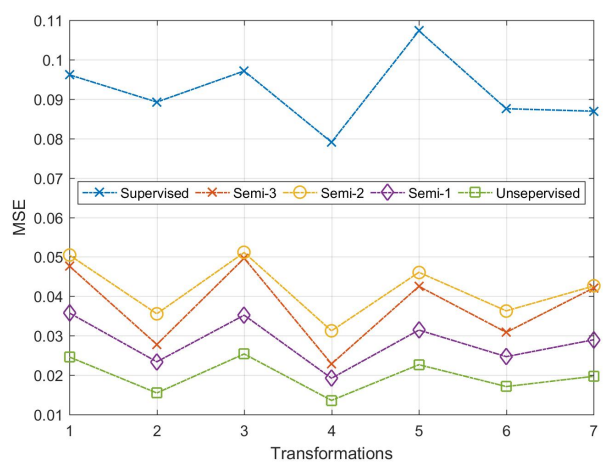

D. Encoding

Figure 4.4: Invariance properties of the learned representation in different layers from five different models: supervised, semi-supervised with three different percentages of supervision (20\% (Semi-1), $30 \%$ (Semi-2), 50\% (Semi-3)), and unsupervised. We applied a range of rotation-based transformations: rotate by $90^{\circ}(1), 180^{\circ}(2), 270^{\circ}(3)$, flip horizontally ${ }^{(4)}$, flip horizontally and rotate by $90^{\circ}{ }^{(5)}, 180^{\circ}{ }^{(6)}$, $270^{\circ}(7)$

\subsubsection{Deep Clustering Through Various Levels of Supervision}

\subsubsection{Supervised Clustering}

To thoroughly investigate the impact of injecting full supervision by providing true labels, we performed extra experiments in four different datasets and analyzed the learned features as well as the impact of embedding the GBAR into the clustering layer of the DCAE. We exploited 
the discriminative patterns provided by supervision components to improve the accuracy of our deep clustering method and allow us to obtain the best representative cluster centres for such data in a given space. Table 4.6 summarizes the performance of our deep clustering method in a fully supervised manner with different settings of the embedded GBAR technique. The results represent the accuracy of clustering. Providing true labels to the DCAE with the embedded clustering method allowed the clustering algorithm to deal with more discriminative features and perform much better compared with other models (i.e. semi-supervised and unsupervised), because the labels' consistency in the latent space can be biased and guided to obtain more characteristic features for compacted groups of such data. In addition, adding supervision guides the clustering task towards more appropriate data partitioning, which allows for the acquisition of the best clustering performance. Table 4.6 also demonstrates that the adoption of the GBAR technique restrains the clustering algorithm from achieving competitive clustering results, because the obtained latent representations are biased and guided by the provided supervision, offering clustering-friendly features for such a clustering algorithm; in other words, imposing more restrictions can be inefficient and confusing.

Table 4.6: Supervised Clustering. Clustering accuracy with GBAR regularization technique using different weighting coefficients.

\begin{tabular}{|r|ccc|c|c|c|c|}
\hline & $c_{\alpha}$ & $c_{\beta}$ & $c_{F}$ & MNIST & USPS & MNIST fashion & SVHN \\
\hline With Regularization & 0.1 & 0.1 & 0 & $84.04 \%$ & $95.51 \%$ & $77.51 \%$ & $75.46 \%$ \\
\hline With Regularization & 0.8 & 0.8 & 0.0003 & $84.78 \%$ & $94.27 \%$ & $77.41 \%$ & $74.39 \%$ \\
\hline With Regularization & 0.8 & 0.8 & 0.000001 & $96.81 \%$ & $93.67 \%$ & $77.37 \%$ & $73.33 \%$ \\
\hline Without Regularization & 0 & 0 & 0 & $\mathbf{9 8 . 8 2} \%$ & $\mathbf{9 5 . 0 6} \%$ & $\mathbf{8 8 . 7 3 \%}$ & $\mathbf{9 2 . 4 0} \%$ \\
\hline
\end{tabular}

\subsubsection{Semi-supervised Clustering}

Here, we created a semi-supervised problem for all experimental datasets by providing two parent-class labels, of which a class label is smaller or larger than 5. This setting allowed us to examine the achievements of our deep clustering method, analyze the learned features with partial supervision information, and observe the impact of adopting the GBAR technique. Although injecting partial supervision into the deep clustering method is supposed to provide discriminative features, the results of Table 4.7 show that the clustering algorithm embedded into a semi-supervised deep clustering method struggles to demonstrate a good clustering performance. However, clustering with the adoption of the GBAR significantly improves the 
clustering accuracy, where adding the regularization technique into the semi-supervised model ensures that each node is specialized and enforces input features to equally spread out over the clustering layer nodes in a discriminative manner. These constraints provide discriminative patterns to enhance the clustering algorithm in more trustworthy partitioning, reveal more accurate compacted groups for the learned feature representations, and thus improve the performance of the clustering algorithm. Therefore, utilizing a small part of supervision as well as the GBAR in this model guides the clustering algorithm in supporting its achievement and help to obtain more appropriate data partitioning.

Table 4.7: Semi-supervised. Clustering accuracy with GBAR regularization technique using different weighting coefficients.

\begin{tabular}{|r|ccc|c|c|c|c|}
\hline & $c_{\alpha}$ & $c_{\beta}$ & $c_{F}$ & MNIST & USPS & MNIST fashion & SVHN \\
\hline With Regularization & 0.1 & 0.1 & 0 & $76.42 \%$ & $62.87 \%$ & $36.58 \%$ & $31.52 \%$ \\
\hline With Regularization & 0.8 & 0.8 & 0.0003 & $\mathbf{9 7 . 7 7} \%$ & $\mathbf{9 1 . 9 2} \%$ & $\mathbf{6 3 . 5 9} \%$ & $\mathbf{4 3 . 9 6} \%$ \\
\hline With Regularization & 0.8 & 0.8 & 0.000001 & $94.60 \%$ & $83.30 \%$ & $55.64 \%$ & $33.74 \%$ \\
\hline Without Regularization & 0 & 0 & 0 & $27.53 \%$ & $28.45 \%$ & $30.98 \%$ & $26.30 \%$ \\
\hline
\end{tabular}

\subsection{Summary}

This study has shown that a deep clustering method is capable of learning discriminative data representations incorporated into different learning schemes, i.e. unsupervised, semisupervised, and supervised, with the help of two regularization techniques. This analytical study seeks to establish an understanding of the effectiveness of the discriminatory power that is obtained by two discriminative attributes: data-driven discriminative attributes with the support of regularization techniques, and supervision discriminative attributes with the support of supervision knowledge. The mechanism of imposing a discriminative power on the clustering layer or injecting it into the body of the learning process makes the learned features derived from the encoding layer the best discriminative attributes, forming a kind of structure that reconciles the one discovered by the clustering process and the one provided by labeling patterns. During our experiments, a well-defined structure for clusters is found through the encoding part of the DCAE, in the presence of discriminative power attributes. An available side of background knowledge along with representative patterns in latent space can be leveraged to find the best partitioning of data and maximize the purity of clusters. We evaluated our experimental methods on MNIST, USPS, MNIST fashion, and SVHN datasets, and show 
the clustering accuracy of our methods through supervised, semi-supervised and unsupervised learning levels, illustrating the influence of discriminatory power on clustering performance. We found that such supervision knowledge greatly helps to form discriminative transformations that are learned by the encoding part of the DCAE model and significantly improves clustering performance. The results also demonstrate that even weak or partial supervision knowledge could significantly improve the quality of deep clustering. Finally, the impact of the regularization techniques on the achievements of clustering performance through a certain level of supervision has been discussed.

In terms of performance, although the DeepCluster method retrieved the effective latent representation into an appropriate approximation of the original input data, it did not perform well with the more challenging datasets, such as the SVHN one, in which large appearance variance and image noise are commonly observed. The reached accuracy is reasonable, as the clustering method intends to group the data examples based on their backgrounds' shape and color. With supervision, the accuracy is increased by more than double when weak labels are provided. We argue that the common structures are not well formed without supervision. Further studies to improve the deep clustering would be beneficial, particularly on more difficult datasets. In this study, we only provided a few limited scenarios involving the injection of supervision; we believe that there is much more scope for the investigation and exploration of various other scenarios for deep clustering with supervision and partial supervision, aiming to differently simulate a shortage of labeled data.

The adoption of the GBAR regularization technique varies with regards to improving the clustering accuracy across numerous learning schemes. It does provide benefits in improving the semi-supervised clustering accuracy, revealing more accurate compacted groups for the learned feature representations. However, it does not significantly affect the clustering results of the unsupervised model and restrains the supervised clustering method from achieving competitive clustering. Thus, it would be of considerable interest to further investigate the relationship between the GBAR regularization technique and invariance properties of the learned representation through different supervision levels. Moreover, further theoretical analysis is also required to understand how to improve clustering performance.

A key observation from work shown in this chapter and the previous chapter is that the characteristic strength of learned representations lies in its ability to enhance and further support the embedded clustering. This observation raises the question of whether the characteristic strength of learned representations aids in compressing and accelerating deep neural networks? 
In Chapters 5 and 6, we explore this idea by evaluating the importance of a network's units for the purpose of network compression. Following this, Chapter 7 concludes by presenting a comprehensive review of time-series data analysis, with emphasis on deep time-series clustering (DTSC), and a founding contribution to the area of DTSC application. The state-of-the-art and outlook on DTSC are also provided. 


\section{Chapter 5}

\section{Reducing Neural Network Parameters via Neuron-based Iterative Pruning}

\section{Contents}

5.1 Introduction $\ldots \ldots \ldots \ldots \ldots \ldots \ldots \ldots \ldots$

5.2 Proposed Method . . . . . . . . . . . . . . . . . . . 99

5.2.1 Importance of Individual Neurons via Majority voting (MV) $\ldots 100$

5.2 .2 Pruning algorithm . . . . . . . . . . . . . . . . 101

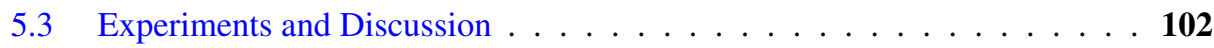

5.3.1 Measuring Neuron Importance via Ablation . . . . . . . . . . . 104

5.3.2 Pruning Redundant Neurons During Training . . . . . . . . 106

5.3.3 Integrating our Pruning Method to Sparsely-Connected Networks

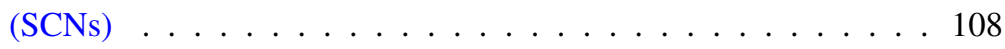

5.3.4 Extension to Convolutional Neural Networks (CNNs) . . . . . . 109

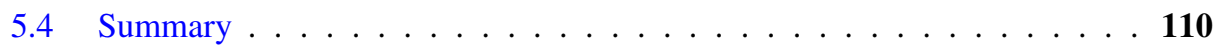




\subsection{Introduction}

The over-parametrized and redundant nature of deep neural networks present significant challenges for many applications. For instance, deploying sizeable deep learning models to a resource-limited device leads to various constrains, as on-device memory is limited [205]. Moreover, training with more parameters than necessary incurs expensive computational costs and high storage requirements. In an attempt to confront these challenges, several approaches have been developed to visually understand the importance of intermediate neurons in neural networks $[70,100,101]$ and measure the influence of hidden units [102, 103,119]. Although these approaches provide different ways to measure the importance of individual hidden units and can be utilized to determine neuron selection criteria for effective pruning, most of the focus is on gaining a better understanding of the network's behavior, with limited attention being paid to pruning studies. The ways in which neuron-based pruning assists in decreasing the complexity of large scale networks are not the focus of the current research. Influential neurons usually identify essential features or high-level concepts on a trained network. Recognizing the importance of such neurons can help to reduce the model complexity by discarding less important units. Reducing the complexity of models while maintaining their powerful performance is always desirable.

Pruning approaches can be applied to any part of deep neural networks, including weights [210, 211,215], neurons [225, 227], filters [228], and channels [207]. Most of the existing methods tend to focus on compressing networks rather than on discovering informative neurons for effective pruning. The fact that not all nodes deliver essential information for the final prediction of the model motivates us to fundamentally rely on applying the importance method when pruning non-informative neurons. Moreover, model compression not only focuses on model parameters, but also on the intermediate activation, which has rarely been studied in previous works. Most of the existing methods also tend to compress the networks through the following three-step procedure: training, pruning, and fine-tuning; in contrast, we train our models from scratch without the use of any pre-training or fine-tuning. We integrate the pruning procedure into the learning process, aiming to find a smaller, well-suited architecture to the target task at the training phase. The main goal of pruning algorithms is to obtain a subnetwork with much fewer parameters without harming accuracy. The pruned version, a subset of the whole model, can represent the reference model at a smaller size with much fewer parameters. Hence, overparameterized networks can be efficiently compressed while maintaining the property of better generalization [44]. 
In this research work, the focus is on a neuron-pruning approach that is carried out according to levels of importance. We propose a majority voting technique which votes for crucial neurons and removes redundant nodes accordingly. Our activation-based method aims to compute a measure of relevance that identifies the most critical neurons by assigning a voting score to evaluate their importance. In order to gather conclusive evidence to evaluate the effectiveness of our method, an experiment based on ablation analysis in trained models was carried out. By comparing our importance method with several baselines, we show that our method substantially outperforms others in terms of the effective measurement of neurons. We also introduce a network-wide holistic approach to prune neurons based on our majority voting method during training, without involving any pre-training or fine-tuning procedures. This proposed framework introduces a mechanism which embeds efficient neuron measurement into the pruning process. This mechanism helps to effectively reduce the models' complexity by eliminating the less important neurons. We evaluated our pruning model on MNIST and CIFAR10 datasets, and the experimental results show that the proposed method efficiently reduces the number of parameters without harming the accuracy.

The rest of the chapter is organized as follows. We describe our proposed methodology in section 5.2 and present our experimental results in section 5.3. Lastly, concluding remarks are provided in section 5.4 .

\subsection{Proposed Method}

In this research work, we introduce a comprehensive approach to prune network's neurons based on our majority voting method during training, without involving any pre-training or fine-tuning procedures. The proposed method introduces a mechanism for measuring the importance of neurons and pruning them accordingly into the body of the learning phase, aiming to obtain a subset of the whole model which represents the reference model with much fewer parameters. In this section, we introduce our overall proposed framework, which consists of two parts. First, the measurement of neuron importance is discussed; this includes utilizing the majority voting approach in order to determine the importance of neurons in each layer. Then, we introduce a network-wide holistic approach which can be used to prune network neurons during training. The details are provided below. 


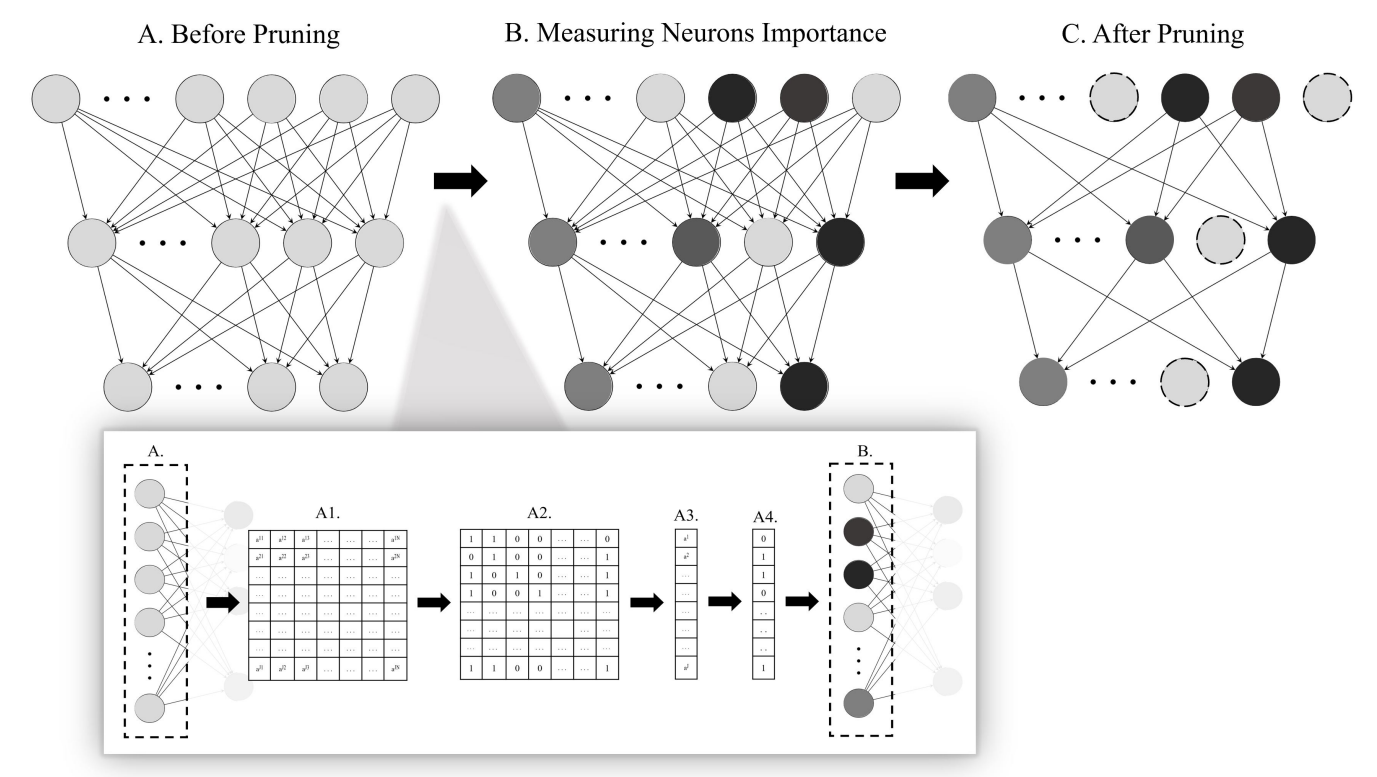

Figure 5.1: Neuron-based pruning method. (A) The initial state of the fully-connected layers. After each training cycle, (B) measuring neuron importance via MV (Fig. 5.2), where the dark circles in the diagram indicate important neurons. (C) Pruning the less important neurons, based on which the income or outgoing connections are removed.

\subsubsection{Importance of Individual Neurons via Majority voting (MV)}

We aim to detect influential neurons in neural networks by evaluating their activation. Feeding the training data through the network, each example is represented differently and has individual activation throughout all neurons in the network. We apply forward passing through an optimized model to find the output of each neuron, called activation. This can be viewed as random variables, and different input images can sample more instances. This measure of importance is discussed in depth below. Each layer has weights that are multiplied with an input example, $x$, to produce an output corresponding to $n$ activation. The activation at the $j$-th neuron is computed as the weighted sum of activations from all neurons in the $i-1$-th layer. The output of the $j$-th unit in the $i$-th layer of the neural network is defined as:

$$
t_{j}^{i} X_{n}=\sigma\left(b_{j}^{i}+\sum_{p} w_{p, j}^{i-1} t_{p}^{i-1} X_{n}\right)
$$

where $X_{n}$ denotes the $n$-th data example at the input, $\sigma$ is the activation function, $b_{j}^{i}$ denotes the corresponding bias for the $j$-th unit in the $i$-th layer, $w_{p, j}^{i-1}$ is the weight that connects $p$-th neuron from the previous layer $i-1$ with the $j$-th unit in the $i$-th layer (existing layer). 
After this, the activation matrix is obtained by Eq.(5.1). For each row, we set the top $l$ largest activation neurons to 1 and others to 0 by using the following form:

$$
v_{j}^{i} X_{n}=\left\{\begin{array}{ll}
1 & \text { if } \operatorname{argsort}\left(t_{j}^{i} X_{n}\right)[1: l] \\
0 & \text { Otherwise }
\end{array} .\right.
$$

As a result, a binary matrix is obtained with $J * N$ dimension in the $i$-th layer, where $J$ is the number of neurons in a layer and $N$ the number of input examples. The obtained matrix determines how important a neuron is for a given example, where 1 indicates the most influential neuron and 0 otherwise. Then, we sum over columns (examples) to score the number of times that the $j$-th neuron is one of the top neurons in the $i$-th layer for given examples, voting for the crucial neurons. This is given by the following form:

$$
y_{j}^{i}=\sum_{n=1}^{N} v_{j}^{i} X_{n} .
$$

We set a $k$ percentage of the $J$ neurons, which have the largest voting scores, to 1 and the remaining to 0 . Here, $k$ denotes the percentage of the largest index of $y$. For every layer, we will come up with a binary vector that indicates whether such neurons are important or not, where 1 denotes that the neuron is important and 0 otherwise. The procedure of our majority voting (MV) method is summarized in Fig. 5.2.

$$
\psi_{j}^{i}=y_{j}^{i}=\left\{\begin{array}{ll}
1 & \text { if } \operatorname{argsort}\left(y_{j}^{i}\right)[1: k * J] \\
0 & \text { Otherwise }
\end{array} .\right.
$$

\subsubsection{Pruning algorithm}

We introduce a method that measures the importance of a network's neurons and prunes them accordingly during training. Our criterion for measuring the value of individual neurons and finding less important ones is critical, as it allows us to effectively identify and prune redundant neurons. As shown in Fig. 5.1, our pruning algorithm starts with standard network architecture and performing standard training. After a training cycle, we measure the neurons' importance, applying our majority voting method as described in the previous section 5.2.1. For every layer, we come up with a binary vector that indicates $k \%$ of important neurons, which is a result of the MV method Eq.(5.4). To eliminate the non-informative neurons, we remove a certain number of neurons that have the lowest voting scores based on the predefined percentage. The complete algorithm is given in Algorithm 3. 


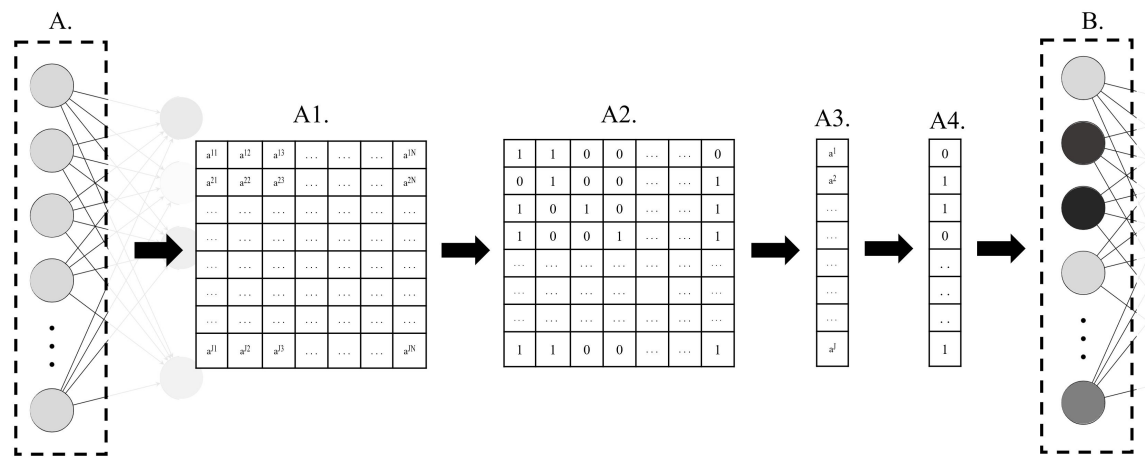

Figure 5.2: Majority voting (MV) method. After each training cycle, we collect the activation for each neuron $j$ and each input example $n$ (A1). The MV method votes for the highest activation scores; a binary matrix is obtained (A2), which determines how important a neuron is, where 1 indicates the most influential and 0 otherwise. Then, we sum over columns (examples) to score the number of times that the $j$-th neuron is one of the top neurons for given examples, voting for the crucial neurons (A3). We set a $k$ percentage of the $J$ neurons, which have the largest voting scores, to 1 and the remaining to 0 . Here, $k$ denotes the percentage of the largest index of $y$. For every layer, we will come up with a binary vector that indicates whether such neurons are important or not, where 1 denotes that the neuron is important and 0 otherwise (B).

Starting with standard training, we begin to apply our pruning algorithm at the start of each training cycle based on certain conditions, including the use of early stopping with the patience of $t$ epochs and the observation of the validation accuracy. In other words, pruning is made under two conditions: every $t$ iterations and when the accuracy is high enough. Pruning neurons at the beginning might lead to the permanent removal of essential neurons; therefore, we start the pruning after each training cycle. We continue to employ our pruning algorithm while surveying the conditions. If we prune neurons in each epoch, the final number of neurons would be too small to maintain reasonable accuracy. This setting allows our model to learn and retain important parameters, which provides our pruning algorithm with valuable guidance to identify non-important neurons and remove them accordingly.

including a certain number $t$ of epochs and the observation of the model's accuracy: could be clearer: under two conditions, pruning is made: every $t$ iterations and accuracy is high enough

\subsection{Experiments and Discussion}

We empirically studied the performance of our proposed method using two different datasets: MNIST and CIFAR10. For fully-connected models, the network architecture consists of three 


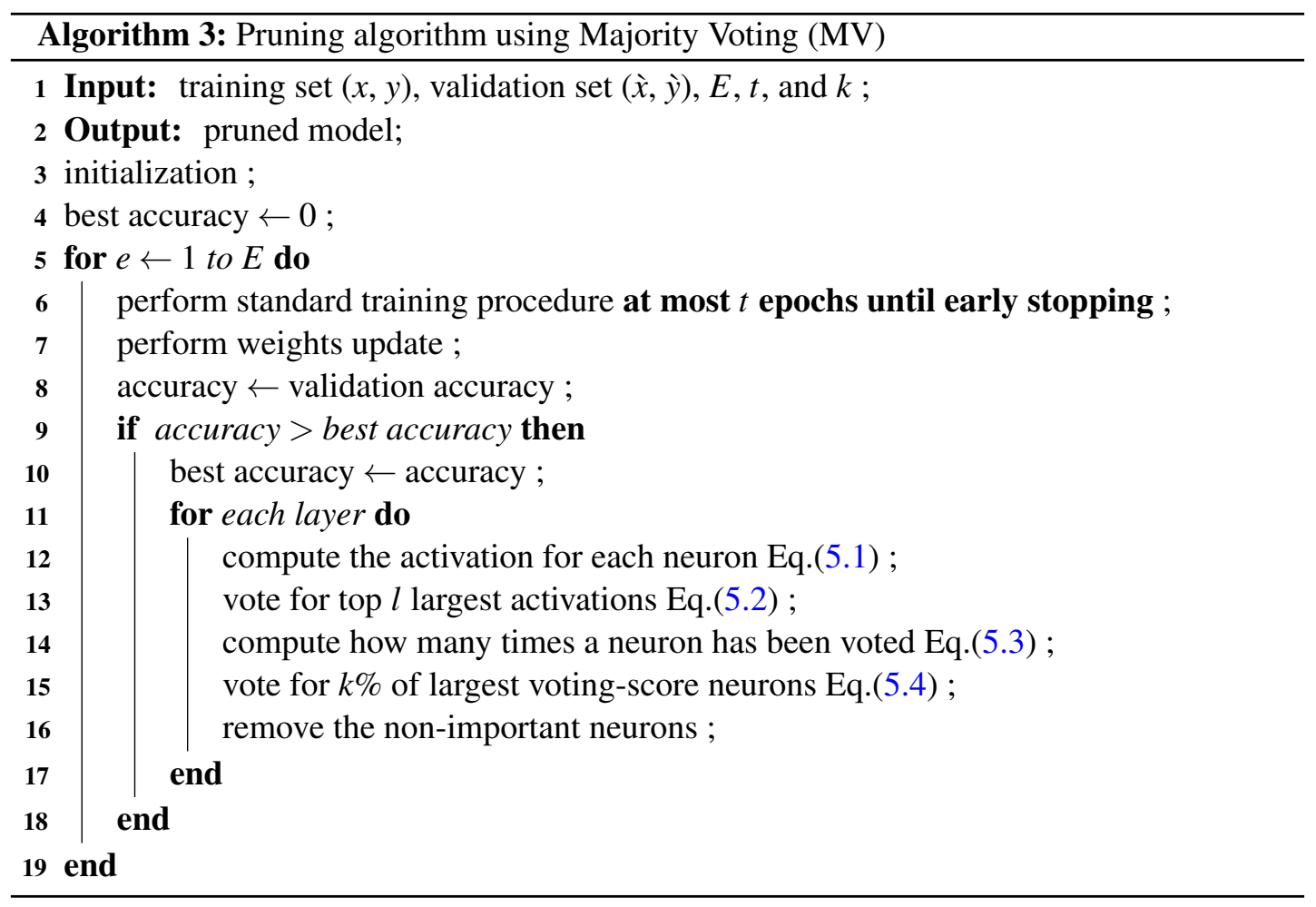

fully-connected layers, which is adopted by the base architecture proposed in [217]. Specifications of the datasets and their architecture are presented in Table 5.1. We first compared our evaluative importance method with several baselines, using an ablation study. The experimental results on both datasets show that our method substantially outperforms the baselines. After this, we applied the proposed method to remove redundant nodes and compress the neuron network during training. Then, we integrated our neuron-pruning method with sparsely-connected network models. The experimental results show that our method adds substantial compression and further reduces the number of parameters, without harming the accuracy. Lastly, we empirically studied our method with convolutional neural networks architecture. The details are provided below.

Table 5.1: Details of Datasets and their FC Architectures used in our experiments.

\begin{tabular}{|r|c|c|c|}
\hline Dataset & Examples & Image Size & FC Architecture \\
\hline MNIST & 70000 & $28 \times 28 \times 1$ & $784-1000-1000-1000-10$ \\
\hline CIFAR10 & 60000 & $32 \times 32 \times 3$ & $3072-4000-1000-4000-10$ \\
\hline
\end{tabular}




\subsubsection{Measuring Neuron Importance via Ablation}

Classification performance was used in order to evaluate the impact of our majority voting method. An ablation study, which is a commonly used technique, allows for the evaluation of the effectiveness of measuring neuron importance quantitatively. This procedure typically refers to the removal of some parts of the model and the study of its performance, as crucial neurons capture meaningful information and contribute substantially to the model's final performance. We ablated unimportant neurons by forcing the activation to be zero and computed the classification accuracy on the test set. Quantifying the effect of the ablation on the classification performance allows for an impartial evaluation in order to measure a neuron's importance and distinguish the most important neurons in a neural network, allowing for layer-wise and whole-network comparisons. This method not only enables the evaluation of neurons' importance, but can also detect the unimportant, redundant neurons, which can be removed while compressing the network during training.

To evaluate the effectiveness of our proposed importance method, it was compared with several baseline methods. These methods are briefly summarized as follows:

- Random. Neurons are randomly ablated.

- Weights sum [225, 228]. Neurons $(p)$ with lowest absolute weights sum values are ablated: $\psi_{p}=\sum_{p}\left|w_{p, j}\right|$.

- Activation Mean [228]. $\psi_{p}=\frac{1}{N} \sum$ mean $\left(t_{p}\right)$, where $t_{p}$ is the activation values for neuron $p$, and $N$ denotes the size of data.

- Activation standard deviation (SD) [228]. $\psi_{p}=\frac{1}{N} \sum s t d\left(t_{p}\right)$.

- Activation $l 1$-norms [228]. $\psi_{p}=\frac{1}{N} \sum\left\|t_{p}\right\|_{1}$.

- Activation 12 -norms [228]. $\psi_{p}=\frac{1}{N} \sum\left\|t_{p}\right\|_{2}$.

All these baseline methods consider neurons with higher values as more important, which is motivated by the intuition that unimportant activation has influential outputs to the final prediction of a model. Following [228], we calculated neurons' importance measure on the activation of the neurons before batch normalization or non-linear activation.

Table 5.2 and Table 5.3 summarize the classification results on CIFAR10 and MNIST test sets respectively. These tables provide layer-wise comparisons, where neuron importance was 
evaluated using different selection criteria in a fully trained model utilizing the ablation approach. A compression ratio of 0.7 was set, where $70 \%$ of the important neurons in each layer are preserved. The tables show layer-wise results for each layer, where we ablated layer by layer and calculated the accuracy for each layer separately. We also examined the whole network cumulative ablation, where the same ratio from all layers in the whole network are ablated. For random selection criteria, the mean value of three runs are reported.

Our ablation study shows that MV achieves higher classification performance compared with other baselines, especially in the case of the whole network cumulative ablation. In CIFAR, Table 5.2, MV achieves $68.28 \%$ when having a compression ratio of 0.7 for each layer of the reference model. This shows that MV has less impact on the dropping of model accuracy compared with the second place method, which used activation standard deviation and achieved $66.33 \%$. This demonstrates the robustness of our proposed method in identifying the most important neurons.

One interesting finding is that ablating neurons with random selection shows that the first layer has stronger negative effects and more synergistic neurons compared with higher hidden layers. It can also be seen that the higher hidden layers are significantly redundant and more class-specific. This observation is consistent with a previous theoretical proposal [275]. One reasonable explanation is that the neuron networks hierarchically learn representations. Hence, the first layer is not relevant to a specific object. Still, it builds feature representations of all input images that are joined to form more relevant object features in the later layers. By ablating these fundamental features, deeper layers fail to produce class-specific features and have more negative impacts on the overall accuracy.

Although a random selection is not robust and not applicable in practice [207], it provides insight and demonstrates that the detection of principal neurons is a critical approach when pruning redundant neurons. The experiment empirically confirms that our importance method is sufficient, given that ablating neurons with low values in the layers only has the most negligible impact on the overall accuracy compared with all baselines. As shown in Table 5.2 and Table 5.3, the experimental results on both datasets show that the method substantially outperforms the baselines. Our proposed method to measure neuron importance helps not only to remove redundant nodes and compress the neuron network, but also to understand their inter-relationships and how said neurons impact the model. The experiment confirms that selecting the right criteria to evaluate neurons' importance throughout all layers can guarantee a successful pruning approach. 
Table 5.2: Examining neuron importance via ablation study with different selection criteria on CIFAR10.

\begin{tabular}{|r|c|c|c|c|}
\hline & 1st Layer & 2nd Layer & 3rd Layer & Cumulative Ablation \\
\hline Random & $45.46 \%$ & $61.84 \%$ & $65.07 \%$ & $21.39 \%$ \\
\hline Weights Sum & $63.75 \%$ & $67.47 \%$ & $67.04 \%$ & $48.62 \%$ \\
\hline Activation Mean & $68.97 \%$ & $68.47 \%$ & $68.48 \%$ & $64.75 \%$ \\
\hline Activation SD & $69.49 \%$ & $68.90 \%$ & $69.22 \%$ & $66.33 \%$ \\
\hline Activation $l 1$-norms & $69.39 \%$ & $68.71 \%$ & $69.32 \%$ & $65.94 \%$ \\
\hline Activation l2-norms & $69.45 \%$ & $68.73 \%$ & $69.31 \%$ & $65.81 \%$ \\
\hline \hline MV & $\mathbf{6 9 . 7 7 \%}$ & $\mathbf{6 9 . 3 9} \%$ & $\mathbf{6 9 . 6 6} \%$ & $\mathbf{6 8 . 2 8} \%$ \\
\hline
\end{tabular}

Table 5.3: Examining neuron importance via ablation study with different selection criteria on MNIST.

\begin{tabular}{|r|c|c|c|c|}
\hline & 1st Layer & 2nd Layer & 3rd Layer & Cumulative Ablation \\
\hline Random & $95.4 \%$ & $97.96 \%$ & $98.41 \%$ & $85.32 \%$ \\
\hline Weights Sum & $95.00 \%$ & $98.39 \%$ & $98.57 \%$ & $94.63 \%$ \\
\hline Activation Mean & $97.88 \%$ & $98.52 \%$ & $98.58 \%$ & $97.98 \%$ \\
\hline Activation SD & $98.58 \%$ & $98.73 \%$ & $98.68 \%$ & $98.44 \%$ \\
\hline Activation l1-norms & $98.56 \%$ & $98.72 \%$ & $98.65 \%$ & $98.40 \%$ \\
\hline \hline Activation l2-norms & $98.51 \%$ & $98.73 \%$ & $98.67 \%$ & $98.37 \%$ \\
\hline MV & $\mathbf{9 8 . 6 8} \%$ & $\mathbf{9 8 . 7 5} \%$ & $\mathbf{9 8 . 7 6} \%$ & $\mathbf{9 8 . 6 8} \%$ \\
\hline
\end{tabular}

\subsubsection{Pruning Redundant Neurons During Training}

The proposed method was implemented using Keras and Tensorflow in Python and evaluated on two computer vision benchmark datasets: MNIST and CIFAR10. The models were trained end-to-end from scratch without involving any pre-training and fine-tuning procedures. All weights were initialized randomly. Stochastic gradient descent optimizer was used, where each batch contained 100 random shuffled images. An initial learning rate of 0.006 with a momentum of 0.9 and weight decay of 0.0002 were used. For our experiments, the value of $t$ was set to be 20 , and the value of $k$ was set to be 0.05 , as a large value of $k$ leads to the removal of many neurons, and the remaining neurons would be too small to maintain reasonable accuracy.

During training, we pruned the network's neurons, after having applied our method to measure the importance of each neuron independently. Consequently, we extended the TensorFlow 
framework to prune the neurons of a network during training. TensorFlow allows us to apply a constraint function to the weights matrix, which in turn means that constraints can be set on network parameters during optimization. For every pruned layer, we utilized the output binary vector, which is obtained via the importance measure method (MV) Eq.(5.4), for the constraint function. The binary vector contributes to generating a binary mask variable which is the same size as the layer's weight matrix. The binary mask determines the participation of the weights in the forward procedure. In the back-propagation, gradients pass through the binary masks, and the masked weights in the forward-propagation are not updated in the back-propagation phase.

Determining and eliminating the non-informative neurons results in a significant additional increment into the body of the learning process. This approach is aimed at forming a kind of structure that enhances the identification of non-informative neurons and removes any redundant parts of the model during training. The comparative results are shown in Table 5.4, and supports our hypothesis that significantly fewer parameters can add enough discriminative ability without harming the original accuracy of the baseline dense models. These are considered a solution to overcoming the over-parametrized and redundant nature of deep learning models. It can be observed that the models' accuracy were improved after the removal of unimportant neurons.

Table 5.4 demonstrates how the pruned versions of models outperform the original, fullyconnected models with only significantly fewer parameters. With regards to the CIFAR10 dataset, it has been shown that a significant gain can be obtained with only $20 \%$ of the weights of the original fully-connected model. Based on CIFAR10-related literature, [276] is considered as one of the state-of-the-art, fully-connected models, which achieves a classification accuracy of $74.1 \%$ with $31,600 \mathrm{~K}$ parameters, while our model reached a comparable accuracy of $74.21 \%$ with only around $4,245 K$ parameters.

Table 5.4: Summarization of our experiments with fully-connected networks.

\begin{tabular}{|r|c|c|c|c|}
\hline & \multicolumn{2}{|c|}{ FC } & \multicolumn{2}{c|}{ MV Pruning } \\
\hline Dataset & Accuracy & $n^{W}$ & Accuracy & $n^{W}$ \\
\hline MNIST & $98.78 \%$ & $2,794 \mathrm{~K}$ & $98.88 \%$ & $232 \mathrm{~K}$ \\
\hline CIFAR10 & $71.90 \%$ & $20,328 \mathrm{~K}$ & $74.21 \%$ & $4,245 \mathrm{~K}$ \\
\hline
\end{tabular}




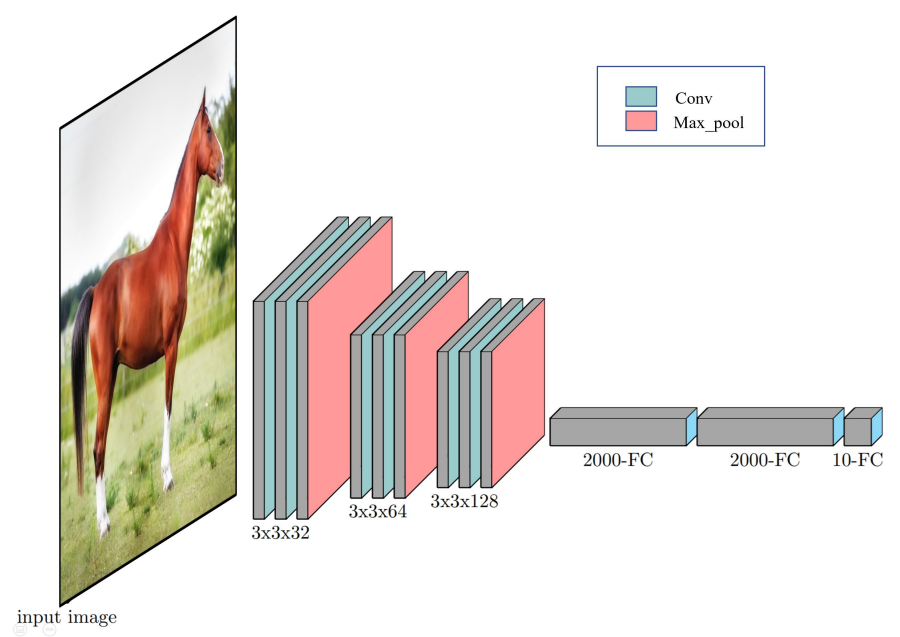

Figure 5.3: The architecture of the CNN model.

\subsubsection{Integrating our Pruning Method to Sparsely-Connected Networks (SCNs)}

To thoroughly investigate the abilities of our proposed method, we also evaluated its performance with sparsely-connected networks (SCNs). SCN by Mocanu et al. [217] is an interesting approach that replaces fully-connected layers with sparsely-connected ones. They have introduced a way to connect nodes in neural networks before training by applying an initial sparse topology based on the Erdôs-Rényi random graph and by starting training using standard optimization techniques. They iteratively replace the weakest connections with the new random initialization until they reach the end of the training process and entirely remove the weakest connections, which leads to a substantial reduction in connections and, therefore, to increased memory and computational efficiency.

A massive number of neurons still poses a challenge, as it can lead to significant redundancy. Although sparsely-connected layers remove unnecessary connections without significant performance degradation, neuron-pruning methods are much more beneficial. This is because unimportant neurons do not contribute much to the final model performance, as shown in the previous section, therefore, all of their income or outcome connections (weights) are trivial and non-informative. Eliminating unimportant neurons can guarantee the removal of extra parameters, as pruning a neuron removes entire rows or columns of the weight matrices from the former and latter layers.

Our experiment began with a sparse topology random graph [217], after which the weakest connections were iteratively replaced with new initialized ones in the training phase. In the 
meantime, we computed a measure of relevance that identified the less critical neurons and pruned them accordingly. Table 5.5 demonstrates that on the MNIST dataset, with our neuronpruning method, we can prune up to $60 \%$ of parameters from the original sparsely-connected models without harming the performance. This supports our hypothesis that pruning unimportant neurons is just as essential as pruning unimportant weights, and that combining both can lead to competitive results, as shown in our findings.

Table 5.5: Summarization of our experiments with sparsely-connected networks.

\begin{tabular}{|r|c|c|c|c|}
\hline & \multicolumn{2}{|c|}{ SC [217] } & \multicolumn{2}{c|}{ MV Pruning } \\
\hline Dataset & Accuracy & $n^{W}$ & Accuracy & $n^{W}$ \\
\hline MNIST & $98.74 \%$ & $89 \mathrm{~K}$ & $98.84 \%$ & $34 \mathrm{~K}$ \\
\hline CIFAR10 & $74.84 \%$ & $278 \mathrm{~K}$ & $75.05 \%$ & $214 \mathrm{~K}$ \\
\hline
\end{tabular}

\subsubsection{Extension to Convolutional Neural Networks (CNNs)}

Convolutional Neural Networks (CNNs) [68] are one of the most famous kinds of neural networks. They replace fully-connected layers with convolution and pooling layers, which significantly decreases the number of parameters. CNN architecture usually comprises convolutional layers for spatially-related feature extraction and fully-connected layers used for classification. However, while CNNs still maintain fully-connected layers, they can additionally benefit from our pruning method.

We studied our pruning method with CNN architectures, where we compressed their fullyconnected layers, as they form the majority of the CNN parameters. For instance, VGG16 [10] comprises $89.40 \%$ of its parameters in fully-connected layers. We adopt the base architecture proposed in [217], which consists of three convolutional blocks, where each block has two convolutional layers with a filter size of $3 \times 3$ with 32 kernels in the first block, 64 kernels in the second block, and 128 kernels in the third block. Each block ends with a max-pooling layer. This is followed by three fully-connected layers consisting of 2000, 2000, and 10 neurons respectively. A standard ReLU activation function was utilized. The detailed architecture of the CNN model is presented in Fig. 5.3. Since the fully-connected layers form $96.60 \%$ of the overall parameters of our architecture, our focus on the fully-connected layers in the convolutional neural networks is justified.

Our experiments were performed on the CIFAR10 dataset. To quantify this, it should be 


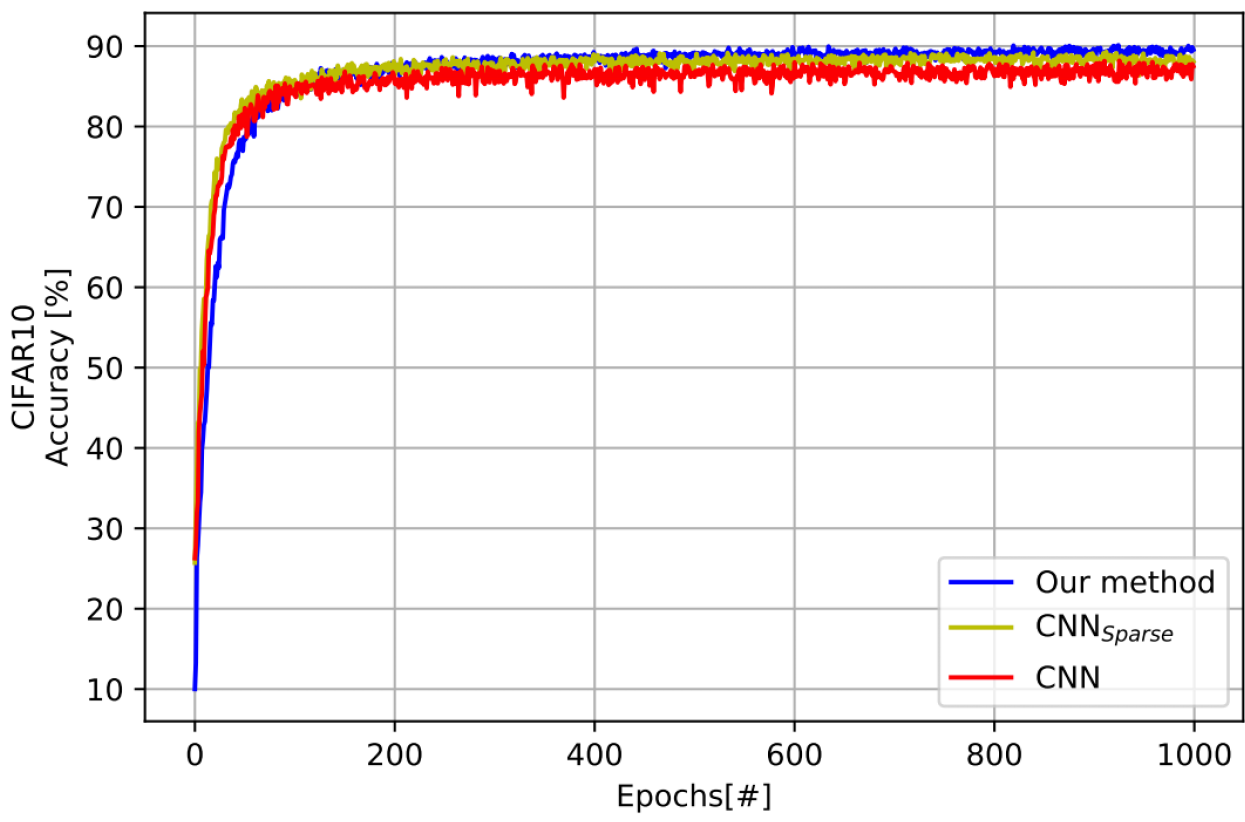

Figure 5.4: Change in accuracy during training with our pruning method for three different models.

noted that our experiment reached a maximum of $90.12 \%$ accuracy, SC achieved a maximum of $89.30 \%$ accuracy, while standard CNN achieved a maximum of $87.65 \%$ accuracy. Each model had 8.407.018, 456.077, and 393.549 weights for CNN, SC and our method, respectively. Our pruned model has shown better accuracy than standard CNN, having removed more than $95 \%$ of its parameters. In other words, with only less than $5 \%$ of the $\mathrm{CNN}$ weights, our method can achieve better accuracy. Fig. 5.4 also shows the changes in validation accuracy in classification tasks with the number of training cycles, which clearly indicates that classification stably converges using an iterative pruning scheme.

\subsection{Summary}

In this research work, we propose an iterative pruning method that prunes neurons based on their level of importance during training. We introduce a majority voting technique to assign a voting score to evaluate neurons' importance, simultaneously identifying the most critical neurons and removing the redundant ones accordingly. The effectiveness of our importance method becomes apparent when compared with several baselines. Empirically, the proposed method is evaluated across various scenarios, including fully-connected networks (FCNs), 
sparsely-connected networks (SCNs), and convolutional neural networks (CNNs) using the MNIST and CIFAR-10 datasets. The experimental results have demonstrated the effectiveness of our pruning method in improving accuracy after removing the unimportant neurons. The results also demonstrate that our proposed method is applicable to weight-based pruning methods and adds extra compression. Moreover, we show that SCNs and CNNs can be pruned into even smaller models using our proposed method with no drop in the reference model accuracy.

Our method mainly targeted the parameters of the fully-connected layers to compress deep neural networks, as most of the weights parameters exist in fully-connected layers. However, most of the computational complexity originates in the convolutional layers due to massive multiplication- and addition operations, although they contain fewer parameters due to parameter sharing. Dealing with an individual CNNs filter requires an intuitive process to determine selective and semantically meaningful criteria for filter selection, where each convolution filter responds to a specific high-level concept associated with different semantic parts. In the following chapter, our core aim is to evaluate filter importance, which provides meaningful insight into the characteristics of the internal representations of neural networks, reducing the computational complexity of the convolutional layers in order to compress and accelerate CNNs. In Chapter 7, we conclude by presenting a comprehensive, detailed review of time-series data analysis and a founding contribution to the area of applying deep time-series clustering (DTSC), with emphasis on identifying state-of-the-art and providing an outlook on DTSC. 


\section{Chapter 6}

\section{Pruning CNN Filters via Quantifying the Importance of Deep Visual Representations}

\section{Contents}

6.1 Introduction $\ldots \ldots \ldots \ldots \ldots \ldots \ldots \ldots \ldots \ldots$

6.2 Proposed Method . . . . . . . . . . . . . . . . . . . 1117

6.2.1 Overview of our Pruning Methodology _ . . . . . . . . . 117

6.2.2 Scoring Channel Importance Method _ . . . . . . . . . . 118

6.2.3 Majority voting (MV) Method . . . . . . . . . . . . 120

6.2.4 Kernels Estimation Method . . . . . . . . . . . . . . . 122

6.3 Experiments and Discussion $\ldots \ldots \ldots \ldots \ldots \ldots \ldots$

6.3.1 VGG16 on CIFAR-10 . . . . . . . . . . . . . . . . . . . . 129

6.3.2 ResNet-20/ResNet-32 on CIFAR-10 . . . . . . . . . . . . . 131

6.3.3 ResNet-20/ResNet-32 on CUB-200 . . . . . . . . . . . 132

6.3 .4 ResNet-50 on ImageNet . . . . . . . . . . . . . . . . . 134

6.3.5 Feature Map Visualization . . . . . . . . . . . . 136

6.3.6 Comparison with Filter Selection Criteria . . . . . . . . . 137

6.3.7 Comparison of Kernal Estimation (KE) vs. Fine-Tuning (FT) . . 142

6.4 Summary . . . . . . . . . . . . . . . . . . . . . . . . 143 


\subsection{Introduction}

Network pruning focuses on discarding unnecessary parts of neural networks, which reduces the massive computational costs and memory requirements associated with deep models. Pruning approaches can be applied to any part of deep neural networks, including fully connected layers [210,211,215, 225, 227, 233] and convolutional layers [48, 207, 228, 229, 232, 233]. The idea of pruning was studied in the early 1990s. Optimal Brain Damage (OBD) by LeCun et al. [210] and Optimal Brain Surgeon (OBS) by Hassibi et al. [211] are considered to be some of the pioneering works in network pruning, demonstrating that several unimportant weights can be eliminated from a trained network with little accuracy loss. Due to expensive computation costs, these methods are not applicable to today's deep models. Obtaining a subnetwork with much fewer parameters without harming accuracy is the main goal of pruning algorithms. The pruned version, a subset of the whole model, can represent the reference model at a smaller size or with much fewer parameters. Thus, overparameterized networks can be efficiently compressed while maintaining the property of better generalization [44].

Recently, Han et al. [215] introduced a simple pruning method to remove connections based on a predefined threshold. Han's framework relies on an iterative pruning procedure to obtain a very sparse model. However, such a non-structured sparse model requires a particular software/hardware accelerator which is not supported by off-the-shelf libraries. Moreover, the reliance on a predefined threshold is less practical and proves too inflexible for some applications. The random connectivity of non-structured sparse models can also cause poor cache locality and jumping memory access, which extremely limits the practical acceleration [23]. In an attempt to confront these challenges, we consider filter-level pruning in our proposed method, where removing the unimportant filter in its entirety does not change the network structure, and the method can be supported by any off-the-shelf deep learning library, allowing for more compression and acceleration by other compression methods, such as the parameter quantization approach [205]. This procedure can also effectively reduce the memory requirements, as model compression focuses on reducing not only model parameters but also the intermediate activation; this has rarely been studied in previous works.

In contrast, filter-level pruning strategies have been widely studied in the community $[48,207,228,229,232]$. The aim of these strategies is to evaluate the importance of intermediate units, where pruning is conducted according to the lowest scores. Li et al. [228] put forward a pruning method based on the absolute weighted sum, where pruning is carried out according to the lowest scores. [229] also proposed a pruning method based on the mean 
gradient of feature maps in each layer, which reflects the importance of features extracted by convolutional kernels. Furthermore, Luo et al. [207] proposed the ThiNet method, which applies a greedy strategy for channel selection. They prune the target layer by greedily selecting the input channel that has the least increase in reconstruction error. The least-squares approach is applied to indicate a subset of input channels which have the smallest impact to approximate the output feature map. These methods tend to compress networks by simply adopting straightforward selection criteria based on the statistical information. However, dealing with an individual CNNs filter requires an intuitive process to determine selective and semantically meaningful criteria for filter selection, where each convolution filter responds to a specific high-level concept associated with different semantic parts.

The fact that not all filters deliver essential information for the final prediction of the model motivates us to fundamentally rely on quantifying the importance of latent representations of CNNs by evaluating the alignment/matching between semantic concepts and individual hidden units to score the semantics of hidden units at each intermediate convolutional layer. Our core aim is to evaluate neuron importance, which provides meaningful insight into the characteristics of the internal representations of neural networks. For the purpose of providing a clear understanding of the internal operation and work mechanisms of deep networks, several approaches have been developed to visually understand convolutional layers [70,96,98], interpret deep visual representations and quantify their interpretability [100,101], as well as measure the influence of hidden units on the final prediction [102-104]. The main focus of these methods is to understand the predictions of a model by analyzing the individual units and seeking an explanation for specific activation. Although these methods provide an intuitive process to determine criteria for neuron selection for effective pruning, most of the previously mentioned methods focus on gaining a better understanding of the network's behavior, with limited attention being paid to pruning methods. Most relevant to our current work is a CNN pruning method inspired by neural network interpretability. Yeom et al. [233] have proposed such a method based on layer-wise relevance propagation (LRP) [104], where weights or filters are pruned based on their relevance score, combining the two disconnected research lines of interpretability and model compression.

In this chapter, we propose a novel framework to compute a measure of relevance, identify the most critical filters and prune the unimportant filters to compress and accelerate CNNs, with only a small drop in model accuracy. Our proposed framework focuses on filter-level pruning based on evaluating the degree of alignment between a semantic concept and individ- 
ual hidden unit representations. Quantifying the interpretability of deep visual representations of CNNs [100] determines the function of individual CNNs filters to deliver essential information, where a filter's feature map is more critical for the network when it represents more information. Individual network decisions can be explained using localization maps [25-27], identifying the important regions in the image for predicting the concept. This fact motivates us to fundamentally rely on applying the feature map importance method when pruning non-informative elements. Our work is considered a pioneering one that attempts to use the quantification of interpretability for more robust and effective CNNs pruning. We first introduce feature maps to detect valuable information and the essential semantic parts, which are fundamental factors in the evaluation of the importance of feature maps.

We propose a more accurate importance measure, a majority voting technique, to compare the degree of alignment values among filters and assign a voting score to evaluate their importance quantitatively. This mechanism helps to effectively reduce model complexity by eliminating the less influential filters and aims to determine a subset of the whole model that can represent the reference model with much fewer parameters. One significant advantage of filter-level pruning is that it does not lead to model structure damage; therefore, other pruning methods can be efficiently adopted for further compression.

To minimize the damage of the pruning procedure, we propose a simple yet effective method to estimate new convolution kernels based on the remaining, crucial channels. Our fundamental insight is that we introduce an optimization problem, based on which the kernels can be estimated depending on the remaining feature maps inputs and the output of the pruned filter. This novel finding differentiates our kernels estimation method from a fine-tuning procedure, which is the most common technique applied by most of the existing methods to recover damaged accuracy.

In order to gather conclusive evidence to evaluate the effectiveness of our method, an experiment based on ablation analysis in trained models was carried out. By comparing our importance method with several state-of-the-art methods, we demonstrate that our approach substantially outperforms others in terms of filters' effective measurement, notably with larger compression ratios. We evaluate our pruning method on CIFAR-10, CUB-200, and ImageNet (ILSVRC 2012) datasets and two types of network architecture: plain CNN (VGG-16 [10]) and residual CNN (ResNet-20/32/50 [11]). The experimental results show that the proposed method efficiently achieves 50\% FLOPs reduction on the VGG-16 model, with only $0.86 \%$ accuracy drop. Although ResNet is more compact and has less redundancy than VGG models, it 
can still reduce $30 \%$ FLOPs, with $0.12 \%$ and $0.02 \%$ accuracy drop on ResNet-20 and ResNet32 respectively. For ResNet-50 on ImageNet, our proposed model is capable of reducing $30 \%$ FLOPs with only a $0.24 \%$ reduction in the original model's top- 1 error and a $0.03 \%$ reduction in the top-5 error.

The rest of the chapter is organized as follows. We describe our proposed methodology in section 6.2, and present our experimental results in section 6.3. Finally, concluding remarks are provided in section 6.4 .

\subsection{Proposed Method}

The chapter mainly studies a filter-level pruning method to reduce model complexity and obtain a small subset of the whole model that can represent the reference model without performance degradation. In this section, we first present our overall pruning framework; our proposed method consists of three major parts, the first two being scoring channel importance via quantifying the importance of individual hidden representations in section 6.2.2 and assigning their voting scores in section 6.2.3, on which we quantitatively evaluate the importance of filters in a specific layer, eliminating the less influential filters accordingly. Then, we introduce a kernels estimation method in section 6.2.4.

\subsubsection{Overview of our Pruning Methodology}

Our method prunes a pre-trained model layer-by-layer with a predefined compression rate. Given a pre-trained CNN model, the proposed novel method is used to compute a measure of relevance that identifies the most critical units. Based on this, the less informative channels are pruned. Then, new convolution kernels are estimated based on the surviving channels, and a final fine-tune for the whole network is carried out. As we mainly focus on filter-level pruning, the pruned version of our model can be further pruned into an even smaller model by adopting other methods. The overall pipeline of our method is presented in Fig. 6.1. Here, the proposed method consists of four iterative steps as follows:

1. Channels Selection Criteria. In contrast to the previous methods, which benefit from the statistical information of layer $i$ to lead the pruning of filters in the same layer, we benefit from the output feature maps of the previous layer $i-1$ to prune filters in the existing layer $i$. Based on the proposed novel method to score channels' importance, we aim to 


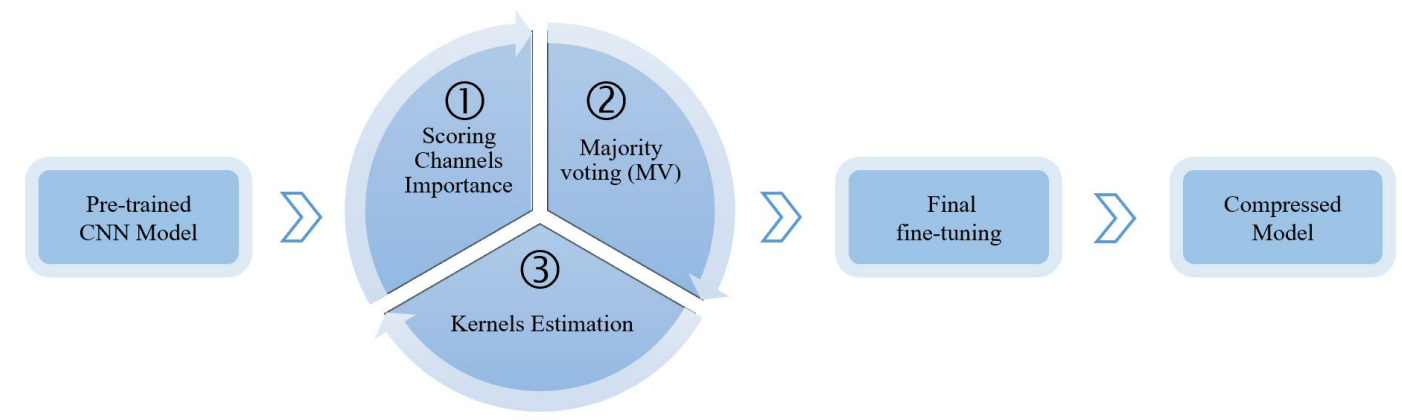

Figure 6.1: The overall pipeline of our proposed framework. A pre-trained CNN model is pruned layer-by-layer through iteratively applying our proposed channels selection criteria, majority voting, and kernels estimation, followed by further final fine-tuning to recover the dropped accuracy.

carefully select a set of channels in layer $i-1$ that are the most influential in the output feature map of layer $i$, as shown in Fig. 6.2.

2. Pruning. Unimportant channels and their corresponding filters in layer $i$ are pruned, keeping the structure of the original network the same. This means that our pruning method assumes that only fewer informative filters and channels can approximate an output feature map of layer $i$. This procedure allows for unimportant filters to be neglected without harming the structure of the original network.

3. Kernels Estimation. To minimize the damage of the pruning procedure, we introduce a method to estimate new convolution kernels based on part of the remaining, un-pruned channels. The target number of filters is obtained by computing a partial convolution, which means that we only utilize the remaining channels of the output feature maps of layer $i-1$ to estimate optimal kernels that approximate the output feature map of layer $i$.

4. We iterate to the first step to prune the next layer.

5. In order to get a more accurate model, further final fine-tuning is carried out once all layers have been pruned.

\subsubsection{Scoring Channel Importance Method}

Our aim was to identify the most influential channels on CNNs based on which the crucial filters are detected. Measuring the importance of every individual convolutional channel al- 

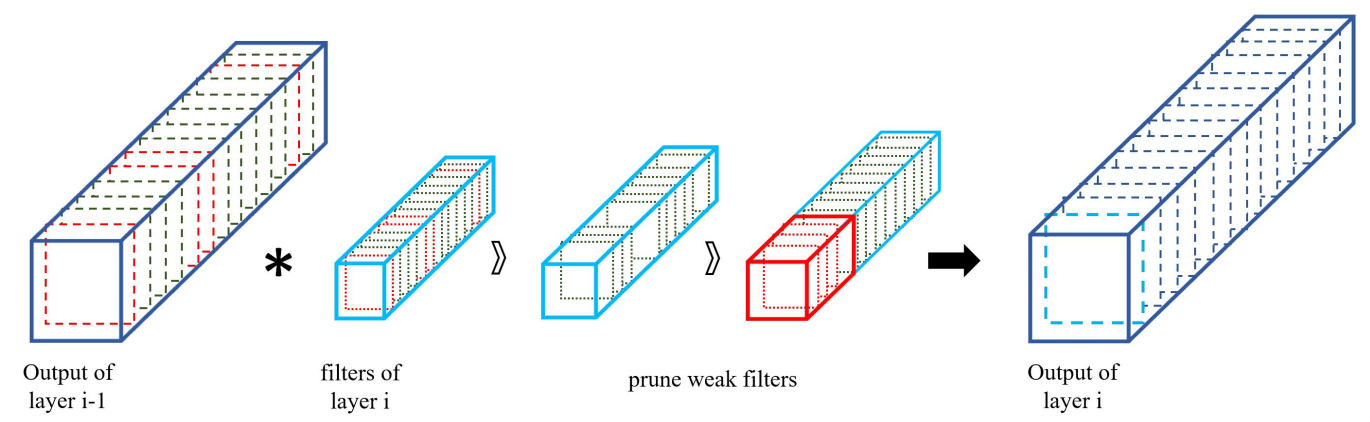

Figure 6.2: Our pruning method. The initial state of a CNN feature map in a fully trained model. Green dotted boxes in the diagram indicate important channels. We identify several weak channels and their corresponding filters (the red dotted boxes), which contribute very little to the overall performance. These channels and their associated filters can be pruned, leading to a pruned model.

lows for the determination of a subset of the channels whose patterns are the most substantial. Inspired by neural network interpretability, we propose a novel pruning framework based on evaluating the degree of alignment between a semantic concept and individual hidden unit representations. Network Dissection [100] was originally developed to quantify the interpretability of latent representations of CNNs that reflect the contribution of an input to deliver essential information for the final prediction of the model.

Every input image $x$ is fed through a pre-trained model by applying forward passing through an optimized model to find the output of each feature map. Each layer has kernels that are convolved on an input example $n$ or a feature map of the internal layers, $x$, to produce an output corresponding to the $n$-th example. The activation at $j$-th feature map is then computed, where the output of the $j$-th unit in the $i$-th layer of CNN is defined as:

$$
t_{j}^{i} x_{n}=\sigma\left(b_{j}^{i}+\sum_{p} w_{p}^{i} * t_{p}^{i-1} x_{n}\right),
$$

where $x_{n}$ denotes the $n$-th data example at the input, $\sigma$ is the activation function (e.g. sigmoid, ReLU), $b_{j}^{i}$ denotes the corresponding bias for the $j$-th unit in the $i$-th layer, $w_{p}^{i-1}$ is the weights of the $p$-th kernel in the $i$-th layer (existing layer), $t_{p}^{i-1} x_{n}$ is the output feature maps of the previous layer $i-1$ and $*$ denotes the $2 \mathrm{D}$ convolution operation.

To measure channels' importance, the latent representations of every individual feature map is evaluated as a solution to a binary segmentation task of the visual concept in the input space. Determining the function of individual filters in a CNN and their ability to localize the meaningful semantic part aids to efficiently measure the importance of different feature maps, 
which is useful for effective pruning. After this, the activation matrix $t_{j}^{i} x_{n}$ is calculated by Eq.(6.1), and the feature map of each internal convolutional channel $j$ is obtained. Then, the distributions of individual feature maps $j$ are computed, and it is based on this that the top quantile value is determined over every spatial location of the feature map. The top quantile value is used as a threshold $T$ to produce a binary matrix for each channel in the latent space. Here, the output feature map $t_{j}^{i} x_{n}$ is thresholded into a binary segmentation $M$, where all regions that exceed the threshold are selected. If a channel in hidden layers has feature maps that are smaller than the input resolution, they are scaled up to match the input resolution using bilinear interpolation. The interpolating function assigns each missing pixel by taking the weighted average of the nearest pixels. We evaluated the importance of every individual channel $M_{j}\left(t_{j}^{i} x_{n}\right)$ by computing intersection over union score between their binary segmented versions against the input binary segmentation $I\left(x_{n}\right)$ of sample $x_{n}$. Fig. 6.3 summarizes the method of scoring channel importance by computing intersection over union (IOU) scores.

$$
I o U_{j}=\frac{\left|M_{j}\left(t_{j}^{i} x_{n}>T\right) \cap I\left(x_{n}\right)\right|}{\left|M_{j}\left(t_{j}^{i} x_{n}>T\right) \cup I\left(x_{n}\right)\right|} .
$$

\subsubsection{Majority voting (MV) Method}

Feeding a set of the data through the network, each example is represented differently and has individual activation throughout all channels in the network. This can be viewed as random variables, and different input images can obtain different $I o U$ scores for different channels. Unlike existing methods that use statistics, we propose a majority voting technique which utilizes a majority voting strategy to vote for crucial channels based on their $I O U$ scores. The majority voting technique compares the IOU scores among all channels and assigns a voting score to quantitatively evaluate the channels' importance and gain more confidence regarding how much each channel contributes to the refined features. Our proposed method aims to compute a measure of relevance that identifies the most critical channels, where it only votes for a channel when all the instances agree, which is what majority voting refers to. After obtaining the $I o U$ scores for each channel $j$, which correspond to an input example $x_{n}$, by Eq.(6.2), our method votes for $l$ values with the highest $I o U$ scores; this is defined as:

$$
v_{j}^{i} x_{n}=\left\{\begin{array}{ll}
1 & \text { if } \operatorname{argsort}(\operatorname{IoU})[1: l] \\
0 & \text { Otherwise }
\end{array} .\right.
$$




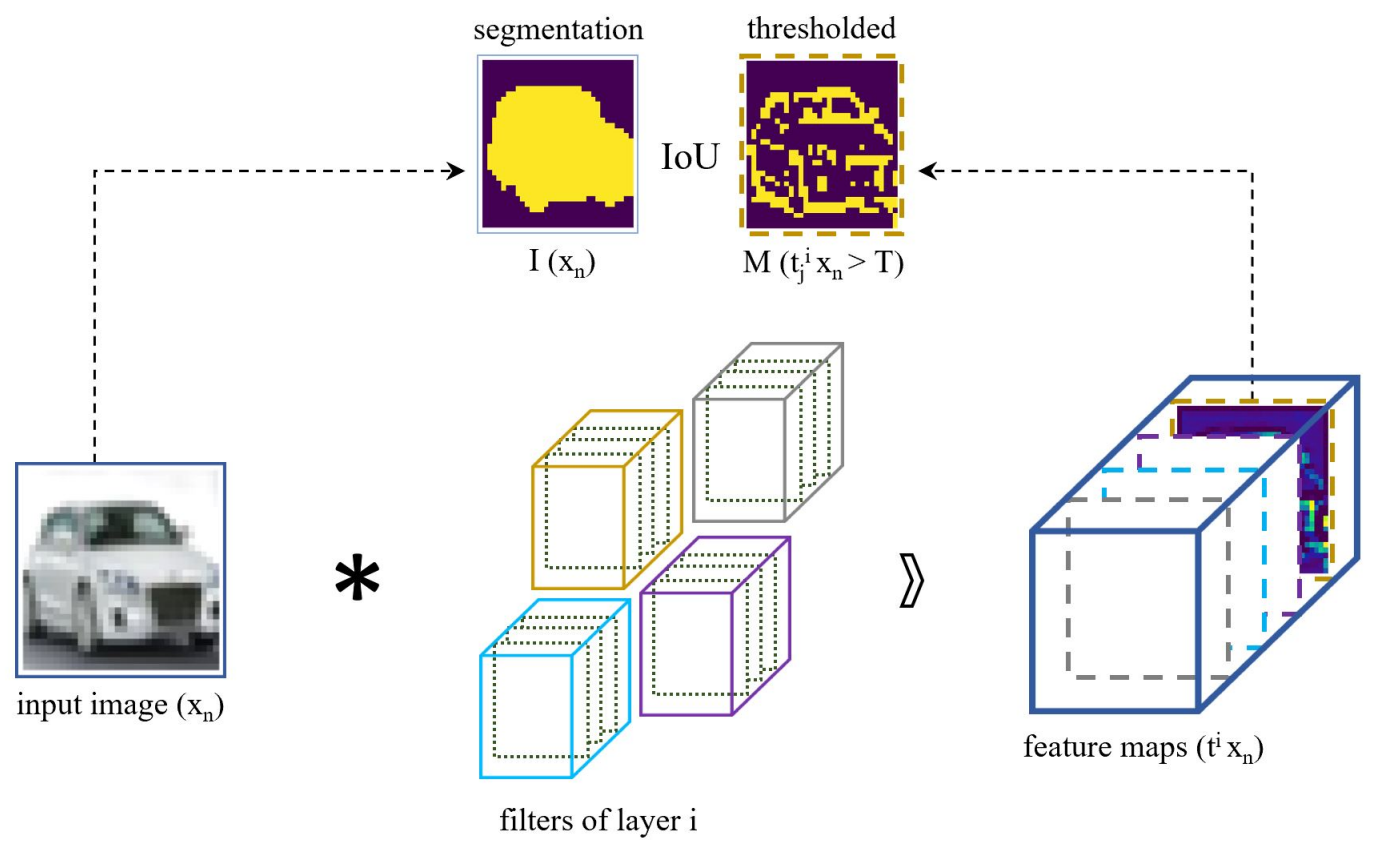

Figure 6.3: Scoring Channel Importance Method. The activation matrix $t^{i} x_{n}$ is obtained by Eq.(6.1). The feature map of each internal convolutional channel $j$ is collected. For each channel $j$, we determined the top quantile value and used it as a threshold $T$ to produce a binary matrix for each channel $M_{j}\left(t_{j}^{i} x_{n}>T\right)$. We evaluated the importance of every individual binary segmentation for each channel $M_{j}\left(t_{j}^{i} x_{n}>T\right)$ against the input binary segmentation $I\left(x_{n}\right)$ by computing intersection over union scores (this figure is best viewed in color).

As a result, a binary matrix is obtained with $J * N$ dimension in the $i$-th layer, where $J$ is the number of channels and $N$ the number of input examples. The obtained matrix determines how important a channel is for a given example, where 1 indicates the most influential and 0 indicates otherwise. Then, we sum over columns (examples) to score the number of times that the $j$-th channel is one of the top channels for given examples, voting for the crucial channels. This is given by the following form:

$$
\begin{gathered}
y_{j}^{i}=\sum_{n=1}^{N} v_{j}^{i} x_{n} . \\
\psi_{j}^{i}=y_{j}^{i}=\left\{\begin{array}{ll}
1 & \text { if } \operatorname{argsort}\left(y_{j}^{i}\right)[1: k * J] \\
0 & \text { Otherwise }
\end{array},\right.
\end{gathered}
$$

where $k$ is the compression rate, and $J$ are the channels. We set a $k$ percentage of the $J$ channels, which have the largest voting scores, to 1 and the remaining to 0 . Here, $k$ denotes the percentage 
A.

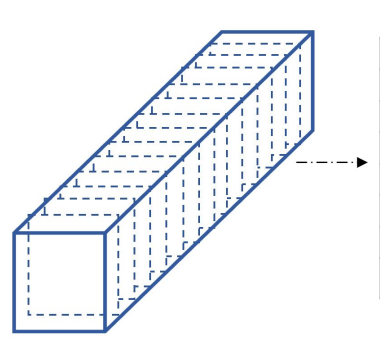

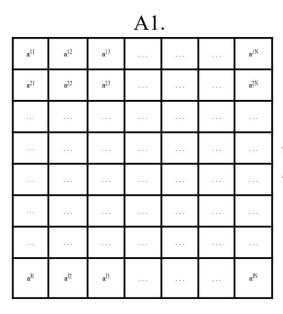

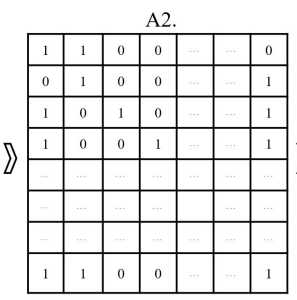

B.

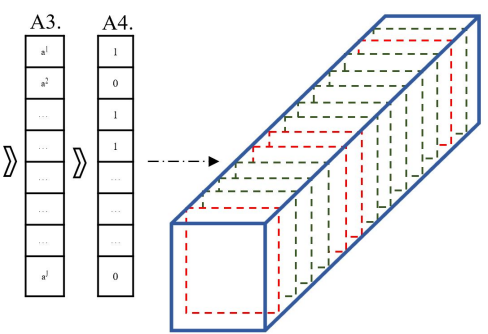

Figure 6.4: Majority voting (MV) method. After obtaining $I o U$ scores for each channel (Fig. 6.3), we collect the $I o U$ for each channel $j$ and each input example $n$ (A1). Our method votes for $l$ percentage of highest $I o U$ scores, and as a result, a binary matrix is obtained (A2), which determines how important a channel is for a given example, where 1 indicates the most influential and 0 indicates otherwise. Then, we sum over columns (examples) to score the number of times that the $j$-th channel is one of the top channels for given examples, voting for the crucial channels (A3). We set a $k$ percentage of the $J$ channels, which have the largest voting scores, to 1 and the remaining to 0 . Here, $k$ denotes the percentage of the largest index of $y$. For every layer, we determine a binary vector that indicates whether such channels are important or not, where 1 denotes that the channel is important and 0 denotes otherwise (B) (this figure is best viewed in color).

of the largest index of $y$. For every layer, we determine a binary vector that indicates whether such channels are important or not, where 1 denotes that the channel is important and 0 denotes otherwise. The procedure of our majority voting (MV) method is summarized in Fig. 6.4.

\subsubsection{Kernels Estimation Method}

A binary vector by Eq.(6.5) indicates $k$ percent of the essential channels for every layer, based on which the non-important channels are pruned. Here, a certain number of channels that have the lowest voting scores are pruned. This mechanism helps to effectively reduce model complexity by eliminating the less influential channels and aims to obtain a subset of the whole model that can represent the reference model with much fewer parameters, whilst preserving the reference model accuracy.

Since there is no guarantee of preserving the accuracy throughout the compression phase, a final fine-tuning or iterative layer-wise fine-tuning are the only techniques applied by most of the existing methods to recover damaged accuracy. A simple compression approach benefits from such valuable steps, especially when the selection criteria is straightforward and does not adequately measure the importance, due to the adoption of less efficient measurement standards.

To minimize the damage of the pruning procedure, we propose a simple yet effective 
method to estimate new convolution kernels based on the remaining unpruned channels. The new kernels can be estimated with only a small number of examples without further training, which is significantly faster to implement. This procedure does not require a multi-step process, in contrast to the fine-tuning procedure (e.g, building a new model, reloading the parameters of the pruned model, and freezing/unfreezing some of the layers), allowing for a fast and efficient process.

Here, we introduce a method to estimate a new convolution kernel based on the remaining, crucial feature map. The new convolution kernel is computed by partial convolution, which means that we will not convolve through all channel in the input feature map, as a subset of channels is pruned already. The target number of filters is obtained by utilizing the remaining channels of the output feature maps of the previous layer to estimate optimal kernels that approximate the output feature map of the existing layer. Therefore, we can prune the filter channels while minimizing the pruning effect.

Using the output feature maps of the previous layer and the convolved version of it, which is the output feature maps of the existing layer, we are able to calculate the convolution kernel. This problem is considered a simple optimization problem in the spatial domain. Given an objective function as follows:

$$
\frac{1}{2}\|h * x-y\|^{2},
$$

where $h$ is the 2D convolution kernel, $y$ is the convolution output feature maps, $x$ is a given output feature maps of the previous layer and $*$ forms the 2D convolution operation. The gradient with respect to the convolution kernel $h$ would be:

$$
\frac{d \frac{1}{2}\|h * x-y\|^{2}}{d h}=\frac{1}{N} \sum_{i=1}^{n} x \otimes(x * h-y),
$$

where $N$ denotes the number of samples used in our estimation method, $\otimes$ denotes the correlation operation and $*$ denotes the convolution operation.

\subsection{Experiments and Discussion}

In this section, we empirically study the performance of our proposed method. Pruning channels with efficient selection criteria along with the majority voting technique indicates that channels with larger voting scores are more important in network performance. We first apply the proposed method to prune two types of network architectures - plain networks 


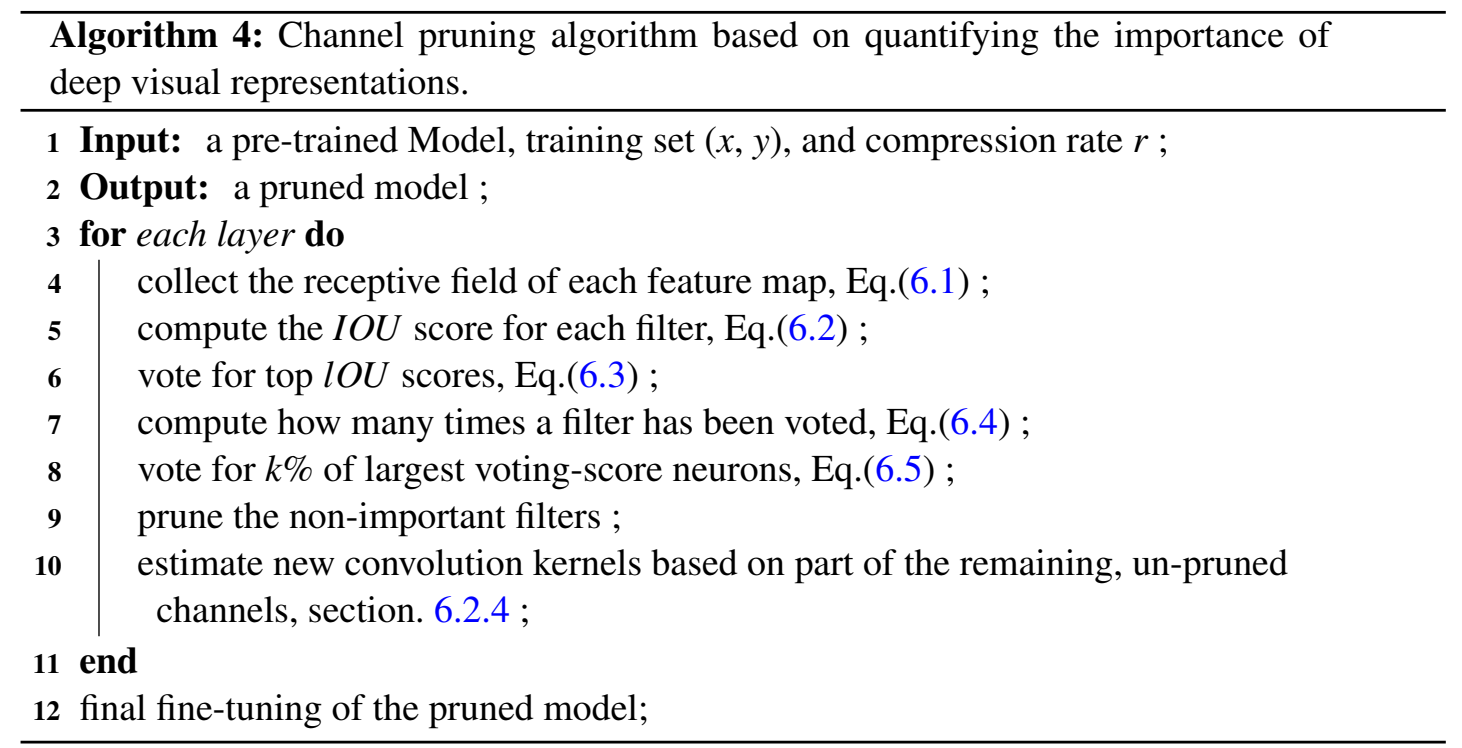

(VGG-16 [10]) and residual networks (ResNet-20/32/50 [11]) - on three different datasets: The CIFAR-10 dataset [277], Caltech-UCSD Birds (CUB-200) dataset [278], and ImageNet (ILSVRC 2012) dataset [279]. The experimental results show that our method adds substantial compression and further reduces model complexity, with little reduction in model accuracy. In this section, we also compare our selection criteria for filter-level pruning with several baselines, and evaluate the change in loss caused by removing a set of filters. The experimental results show that our method substantially outperforms all other baselines. Finally, we empirically verify the validity of our kernels estimation (KE) method and compare it with the standard fine-tuning (FT) procedure. The proposed method was implemented using Keras [265] and Tensorflow [52] in Python.

\section{Experimental Datasets}

We evaluated our filter-level pruning method on three different datasets.

- CIFAR-10 [277] : is an image dataset which consists of 60,000 images. Each example is a 32 x 32 color image, and is associated with a label from 1 of 10 different classes. Each class contains 6,000 images. The CIFAR-10 consists of 50,000 examples as a training set and 10,000 examples as a test set.

- CUB-200 [278] : is a bird subcategories image dataset which contains 200 species of birds; 11,788 images are associated with a label from 1 of 200 classes, where each class 
has roughly 30 training images and 30 testing images. The CUB-200 contains 5,994 examples as a training set and 5,794 examples as a test set.

- ImageNet (ILSVRC 2012) [279] : is a large-scale dataset which consists of over 14 million labeled images. Each example is associated with a label from 1 of 1,000 different classes. The ImageNet consists of 1.28 million images as a training set and 50,000 images as validation images.

For CUB-200 and ImageNet, each image is resized to $256 \times 256$, then a $224 \times 224$ area is randomly cropped from each resized image. The classification performance is reported on the test set for both CIFAR-10 and CUB-200 datasets and on the standard validation set for the ImageNet dataset.

\section{Inputs and Feature Maps Binary Segmentation}

To collect instances for channel selection, we randomly selected ten images from each class in the training set to form our evaluation set. These selected samples were used to find the optimal channel subset via Algorithm 4. For semantic segmentation, we used the PSPnet model by [79] to segment the input images of CIFAR-10. For CUB-200, the segmentation masks were provided by Ryan Farrell ${ }^{1}$. We also used the segmentation masks provided by [280] for the ImageNet dataset. The proposed method evaluates every individual convolutional unit in a $\mathrm{CNN}$ as a solution to a binary segmentation task of the visual concept in the input space (Fig. 6.3). Feeding the selected instances through the network, each example has an individual activation throughout all feature maps in the network. Each collected feature map is converted into a binary matrix using the top quantile value as a threshold $T$. An experiment based on different settings to determine the top quantile level $T$ in Eq.(6.2) was carried out in order to gather conclusive evidence to carefully choose the top quantile value when producing a binary matrix for each channel in the given feature map. The comparative results are shown in Fig. 6.5. As a result, the top quantile value is determined such that $M_{j}\left(t_{j}^{i} x_{n}>T\right)=0.8$ over every spatial location of the feature map. Therefore, the output feature maps of the previous layer $i-1$ are segmented into binary segmentation.

\footnotetext{
${ }^{1}$ http://www.vision.caltech.edu/visipedia/CUB-200-2011.html
} 


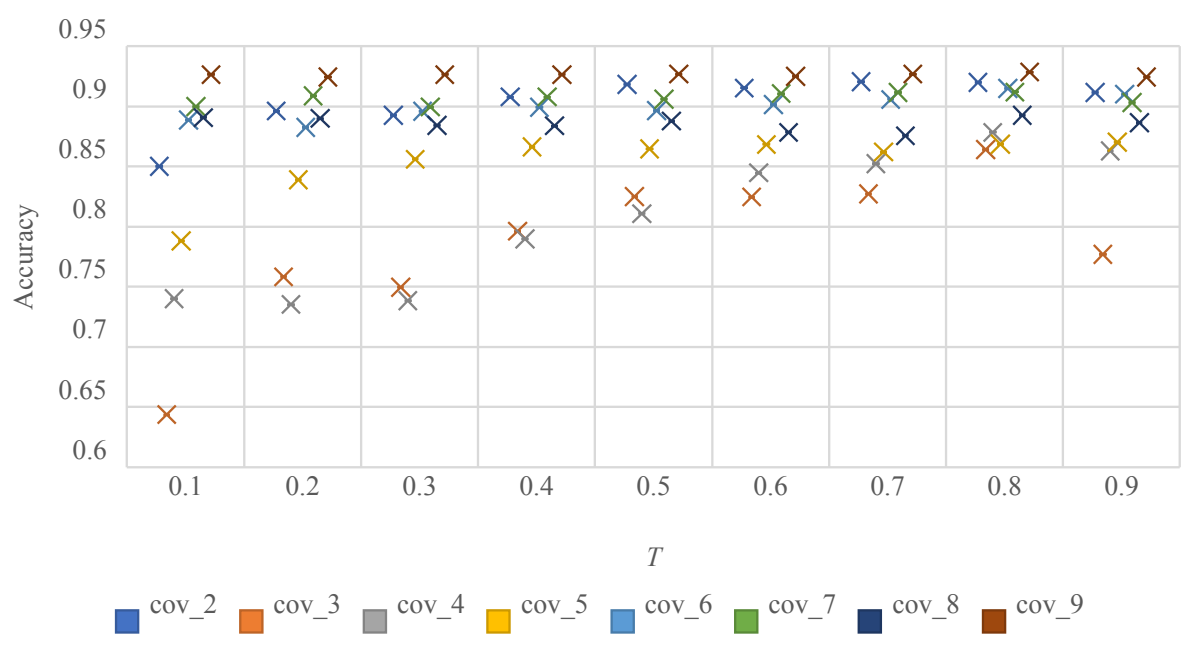

Figure 6.5: Different settings to determine the top quantile level $T$ in Eq.(6.2) at different layers of VGG16 on CIFAR-10.

\section{Implementation Details}

To measure channels' importance, the feature maps' binary segmentation are evaluated against the semantic input segmentation by computing intersection over union ( $I o U)$ score. Given $I o U$ scores for each channel, our results show that each example has different $I o U$ scores, as each is represented differently and has individual activation throughout all channels in the network. Therefore, by using a set of data samples to find the optimal channel subset, the judgment of the selection criteria becomes more accurate. Empirical evidence comes from a comparison between a range of hyper-parameter settings of the $l$ values in Eq.(6.3). Fig. 6.6 presents valuable evidence for choosing 0.2 as an appropriate value for the parameter $l$. The sensitivity of pruning channels for VGG-16 on CIFAR-10 was examined with minimum MV values, summed $I o U$ scores, and maximum MV values. Fig. 6.7 shows the comparison of different $I o U$ measured criteria and a reduction in the accuracy of different convolution layers, differentiating all three methods. Our proposed method (MV) votes for the highest $I o U$ scores, compares these scores among all examples, and assigns a voting score to compute a measure of relevance that identifies the most critical channels. It only votes for a channel when all instances agree. Fig. 6.7A. shows how the minimum voting scores indicate the most crucial channels. Pruning by smallest voting scores yields better performance than pruning by largest voting scores. As shown in Fig. 6.7C., pruning channels with the maximum MV values causes the accuracy to drop quickly as the pruning compression rate increases. However, from the comparison be- 


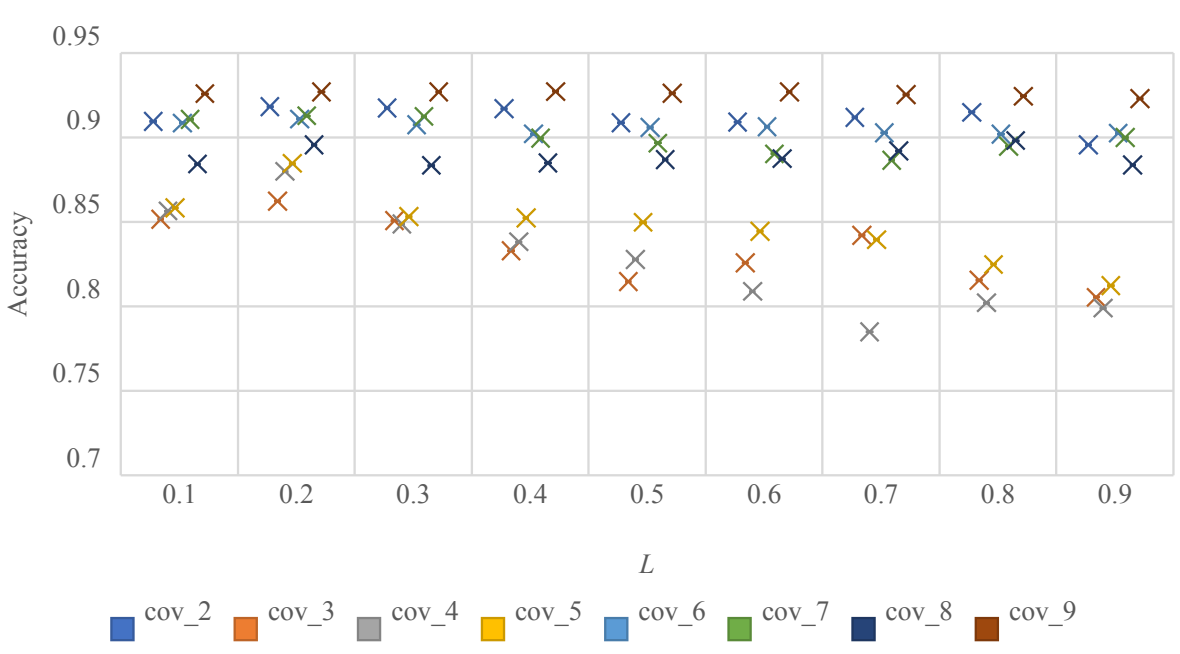

Figure 6.6: Different settings to determine the optimal $l$ value in Eq.(6.3) at different layers of VGG16 on CIFAR-10.

tween pruning with minimum voting scores and the minimum $I o U$ scores summed values, we can see that the accuracy of a pruned network with minimum MV scores adequately evaluates channel importance and demonstrates the best performance.

In each convolutional layer, the filter channels with the smallest voting scores are pruned; consequently, filters and their corresponding channels on a batch normalization layer are also eliminated. After pruning the unimportant filters, we were able to minimize the pruning impact by applying our kernel estimation method. When unimportant filters are discarded, a new model with thinner filters is created. The weights of the modified layers, as well as the nonpruned layers, were transferred to the new model. After pruning all layers, a final fine-tuning for the whole pruned model was performed to recover the overall dropped accuracy. During fine-tuning, the stochastic gradient descent optimizer was used, where each batch contained 32 randomly shuffled images. A data augmentation technique was applied using simple transformations such as flipping images horizontally. The entire network was pruned layer-by-layer. For both CIFAR-10 and CUB-200 datasets, we fine-tuned the pruned models for 40 epochs with a constant learning rate of $10^{-3}$ with a momentum of 0.9 ; a weight decay of 0.0005 was used. This was performed at the last round. For the ImageNet dataset, images were resized to $256 \times 256$, after which we randomly cropped them to $224 \times 224$. The pruned models were fine-tuned for only 20 epochs to reduce training time, where the learning rate changes from $10^{-3}$ to $10^{-5}$. Other parameters were kept the same. 


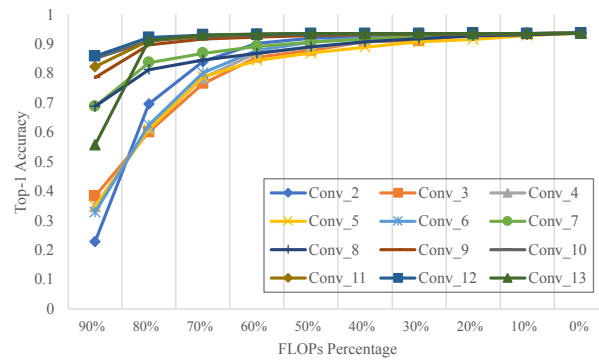

A. Minimum MV values

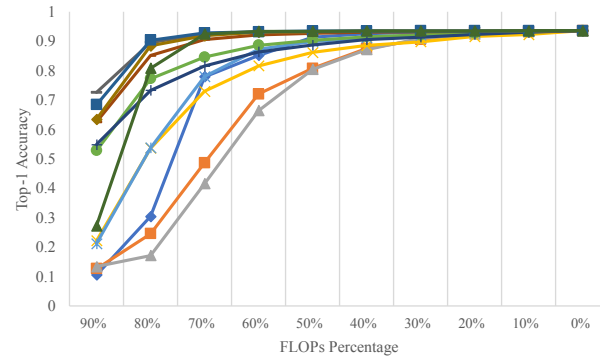

B. Minimum Summed IOU scores

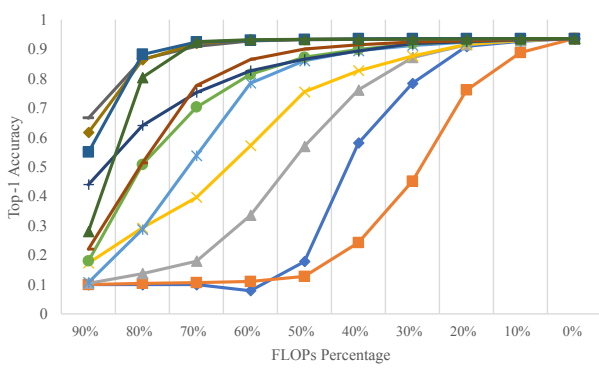

C. Maximum MV values

Figure 6.7: Comparison of $I o U$ pruning selection criteria. (a) and (c) compare our MV of $I o U$ pruning selection criteria when pruning the lowest and highest MV scores. (b) Pruning filters based on assuming IoU scores.

\section{Compression Ratio}

Deciding the number of filters which must be pruned from each layer as well as the best pruning ratios for different layers is a very challenging task [207]. It is also challenging to determine the layer importance due to the fact that the performance of a CNN model is susceptible to specific layers and different layers have a different degree of filter-level redundancy. Thus, we applied a fixed compression ratio to all layers in the pruned model for simplicity. In our experiment, we applied three different compression ratios: pruned-70, pruned-50, and pruned30 , where $70 \%, 50 \%, 30 \%$ of filters are preserved in each convolutional layer, respectively. Reducing the complexity of models with larger compression ratios while maintaining their powerful performance is always desirable, as the inference speed is highly essential for some real-world applications. For example, the model used for self-driving vehicles must return fast predictions for safety considerations. Thus, the FLOPs of this kind of model should be reduced to fulfil the standard requirements. 


\subsubsection{VGG16 on CIFAR-10}

In this section, we evaluate the performance of the proposed method on the most popular deep convolutional network: VGG-16 [10]. VGG-16 is a CNN architecture for large-scale image recognition proposed by Simonyan et al., which was initially designed for the ImageNet dataset. VGG-16 was modified by Liu et al. [281] to fit the CIFAR-10 dataset, achieving state-of-the art results. VGG16 on CIFAR-10 consists of thirteen convolutional layers with a filter size of $3 \times 3$ with a stride of 1 , and a pooling region of $2 \times 2$ without overlap. This is followed by two fully-connected layers, with the last layer consisting of 10 neurons. Due to the smaller input size, the dimensions of the fully-connected layers are shrunk, which significantly reduces the number of parameters. Here, we adopted the model described in [281], adding a batch normalization layer [55] as well as a dropout layer [56] after each convolutional layer. The detailed architecture of the CNN model is presented in Table 6.1.

Table 6.1: VGG-16 on CIFAR-10 and three different pruned models. The number of remaining feature maps and the reduced percentage of FLOPs from each pruned model are shown. VGG-16-pruned-A, VGG16-pruned-50/B and VGG-16-pruned-C are different pruned versions of the original VGG-16.

\begin{tabular}{|c|c|c|c|c|c|c|c|c|c|c|}
\hline & \multicolumn{4}{|c|}{ VGG-16 } & \multicolumn{2}{|c|}{ VGG-16-pruned-A } & \multicolumn{2}{|c|}{ VGG-16-pruned-50/B } & \multicolumn{2}{|c|}{ VGG-16-pruned-C [228] } \\
\hline layer type & $w_{i} \times h_{i}$ & \#Maps & \#FLOP & \#Params & \#Maps & pruned $\%$ & \#Maps & pruned $\%$ & \#Maps & pruned\% \\
\hline Conv 2 & $32 * 32$ & 64 & $3.80 \mathrm{E}+07$ & $3.7 \mathrm{E}+04$ & 38 & $40 \%$ & 32 & $50 \%$ & 32 & $50 \%$ \\
\hline Conv 3 & $16 * 16$ & 128 & $1.90 \mathrm{E}+07$ & $7.4 \mathrm{E}+04$ & 102 & $20 \%$ & 64 & $50 \%$ & 128 & $0 \%$ \\
\hline Conv 4 & $16 * 16$ & 128 & $3.80 \mathrm{E}+07$ & $1.5 \mathrm{E}+05$ & 102 & $20 \%$ & 64 & $50 \%$ & 128 & $0 \%$ \\
\hline Conv 5 & $8 * 8$ & 256 & $1.90 \mathrm{E}+07$ & $2.9 \mathrm{E}+05$ & 230 & $10 \%$ & 128 & $50 \%$ & 256 & $0 \%$ \\
\hline Conv 6 & $8 * 8$ & 256 & $3.80 \mathrm{E}+07$ & $5.9 \mathrm{E}+05$ & 205 & $20 \%$ & 128 & $50 \%$ & 256 & $0 \%$ \\
\hline Conv 7 & $8 * 8$ & 256 & $3.80 \mathrm{E}+07$ & $5.9 \mathrm{E}+05$ & 205 & $20 \%$ & 128 & $50 \%$ & 256 & $0 \%$ \\
\hline Conv 8 & $4 * 4$ & 512 & $1.90 \mathrm{E}+07$ & $1.2 \mathrm{E}+06$ & 410 & $20 \%$ & 256 & $50 \%$ & 256 & $50 \%$ \\
\hline Conv 9 & $4 * 4$ & 512 & $3.80 \mathrm{E}+07$ & $2.4 \mathrm{E}+06$ & 256 & $50 \%$ & 256 & $50 \%$ & 128 & $75 \%$ \\
\hline Conv 10 & $4 * 4$ & 512 & $3.80 \mathrm{E}+07$ & $2.4 \mathrm{E}+06$ & 205 & $60 \%$ & 256 & $50 \%$ & 128 & $75 \%$ \\
\hline Conv 11 & $2 * 2$ & 512 & $9.40 \mathrm{E}+06$ & $2.4 \mathrm{E}+06$ & 205 & $60 \%$ & 256 & $50 \%$ & 128 & $75 \%$ \\
\hline Conv 12 & $2 * 2$ & 512 & $9.40 \mathrm{E}+06$ & $2.4 \mathrm{E}+06$ & 205 & $60 \%$ & 256 & $50 \%$ & 128 & $75 \%$ \\
\hline Conv 13 & $2 * 2$ & 512 & $9.40 \mathrm{E}+06$ & $2.4 \mathrm{E}+06$ & 205 & $60 \%$ & 256 & $50 \%$ & 128 & $75 \%$ \\
\hline $\mathrm{FC}$ & 1 & 512 & $2.60 \mathrm{E}+05$ & $2.6 \mathrm{E}+05$ & 512 & $0 \%$ & 512 & $0 \%$ & 512 & $0 \%$ \\
\hline $\mathrm{FC}$ & 1 & 10 & $5.10 \mathrm{E}+03$ & $5.1 \mathrm{E}+03$ & 10 & $0 \%$ & 10 & $0 \%$ & 10 & $0 \%$ \\
\hline Total & & & $3.13 \mathrm{E}+08$ & $1.47 \mathrm{E}+07$ & & $43.04 \%$ & & $50 \%$ & & $53.03 \%$ \\
\hline
\end{tabular}

Table 6.2 shows the results of the pruned models for VGG-16 on CIFAR-10, VGG16pruned-70, VGG16-pruned-50, and VGG16-pruned-30, in which 70\%, 50\%, and 30\% of filters are preserved respectively in each convolutional layer. This also means that we assigned constant compression ratios of $30 \%, 50 \%$, and $70 \%$ respectively for all layers. Fig. 6.8A. shows that the convolutional layers with 512 feature maps have less impact on the dropping of model accuracy, as they can be pruned up to $70 \%$ without harming the original accuracy. One definite explanation is that small dimensions of feature maps do not indicate meaningful spatial features for these convolutional layers. Our kernel estimation method can recover the pruning 
Table 6.2: Performance of pruning VGG16 on CIFAR-10 using different pruning rates. The classification error is reported.

\begin{tabular}{|l|l|l|l|}
\hline Model & Error(\%) & FLOPs & Pruned \\
\hline VGG-16 [10] & 6.41 & $3.13 \mathrm{E}+08$ & - \\
VGG-16-pruned-70 & $\mathbf{6 . 1 8}$ & $2.20 \mathrm{E}+08$ & $30 \%$ \\
VGG-16-pruned-A & 6.37 & $1.37 \mathrm{E}+08$ & $43.04 \%$ \\
VGG-16-pruned-50/B & 7.27 & $1.57 \mathrm{E}+08$ & $50 \%$ \\
VGG-16-pruned-C & 7.00 & $1.66 \mathrm{E}+08$ & $53.03 \%$ \\
VGG-16-pruned-30 & 9.10 & $9.42 \mathrm{E}+07$ & $70 \%$ \\
VGG-16-pruned-A scratch-train & 8.57 & $1.37 \mathrm{E}+08$ & $43.04 \%$ \\
VGG-16-pruned-50 scratch-train & 9.79 & $1.57 \mathrm{E}+08$ & $50 \%$ \\
\hline
\end{tabular}

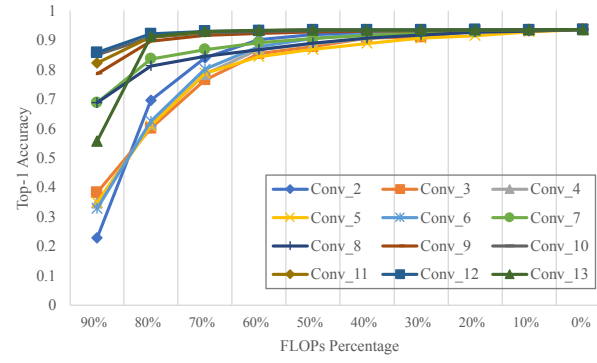

A. Pruning smallest value

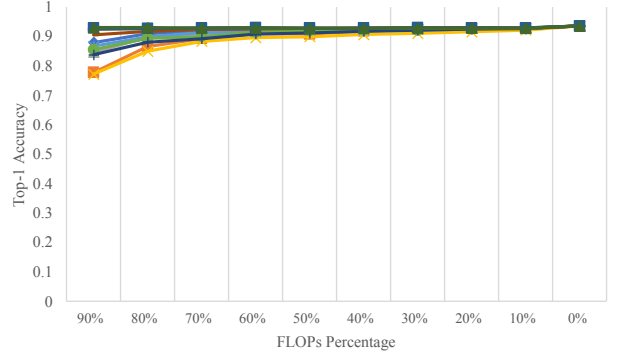

B. Prune and re-estimate new kernels

Figure 6.8: Layer-wise pruning of VGG-16 on CIFAR-10. (a) Pruning filters with the lowest MV scores and their corresponding test accuracies on CIFAR-10. (b) Prune and estimate new kernels for each single layer of VGG-16 on CIFAR-10.

effect and help us to safely prune the majority of the filters of such layers, see Fig. 6.8B.. We observe that the first few layers have stronger negative effects and more synergistic filters compared with higher hidden layers due to hierarchically learnt representations of deep networks. Therefore, an effective pruning method, as well as the reduction of FLOPs, mostly relies on the layer where pruning is applied within the network.

This observation motivated us to assign different compression rates to different layers based on our ablation study; thus, if the layer shows more sensitivity to pruning, the compression ratio decreases. The network pruning ratio is $43.04 \%$ and $53.03 \%$ for VGG16-pruned-A and VGG16-pruned-C, respectively. The detail specification of such pruned models is presented in Table 6.1. Moreover, for both VGG16-pruned-A and VGG16-pruned-70 pruned models, we achieved $43.04 \%$ and $30 \%$ FLOP reduction, respectively, with no drop in the original model accuracy. We also trained a model from scratch with the same architecture as VGG-16-pruned-A 
and VGG-16-pruned-50, which allowed us to obtain the baseline accuracies for such networks and differentiate between training from scratch and pruning. Table 6.2 shows that VGG-16pruned-A scratch-train and VGG-16-pruned-50 scratch-train present considerably worse results than our pruned models. Thus, a model may need a certain level of redundancy during model training to guarantee excellent quality performance. Hence, decreasing a model's size after training can be an effective solution.

\subsubsection{ResNet-20/ResNet-32 on CIFAR-10}

The performance of our pruning method was also evaluated on the famous CNN architecture ResNet [11]. The ResNets for CIFAR-10 have three stages of residual blocks, where $32 \times 32$, $16 \times 16$, and $8 \times 8$ are the sizes of their corresponding output feature maps. Each stage has an equal number of residual blocks. Identity shortcuts are directly used when the input and output comprise the same dimensions. When a feature map's size is down-sampled, the shortcut performs by $1 \times 1$ kernels. This procedure overcomes the issue which occurs when the shortcuts go across feature maps of two different sizes. As the input and output feature map sizes of this convolutional layer are different, we skipped those layers and pruned the remaining layers at each stage.

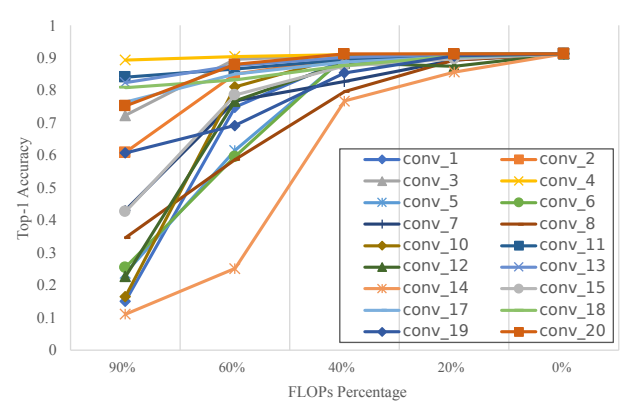

A. ResNet-20

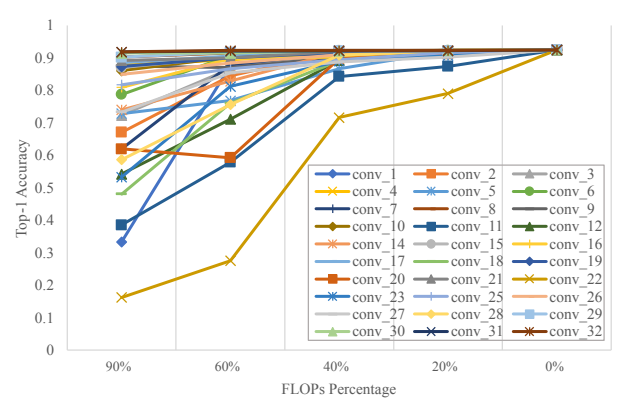

B. ResNet-32

Figure 6.9: Layer-wise pruning of ResNet-20/32 on CIFAR-10.

To investigate the abilities of our proposed method, we chose ResNet-20 and ResNet-32 to represent the ResNet family, which have the same designs, except for the number of layers and the depth of the network. In the initial experiment, we started with a trained Keras implementation with classification errors of $8.75 \%$ and $7.51 \%$ on the test set for ResNet-20 and ResNet-32, respectively. Fig. 6.9 shows the classification accuracy of ResNet-20 (left) and ResNet-32 (right) after pruning each layer using our proposed method. Unlike VGG-16, 
ResNet is more compact, and due to its reduced redundancy, pruning a large number of channels appears to be more challenging. It can be seen that some layers were more sensitive to pruning, such as layers 11 and 22 in ResNet-32 and 14 in ResNet-20. Similar to VGG-16, we found that deeper layers of the ResNet architecture were less sensitive to pruning than those in the earlier layers of the network.

Table 6.3: Performance of pruning ResNet-20/32 on CIFAR-10 using different pruning rates. The classification error is reported.

\begin{tabular}{|l|l|l|l|}
\hline Model & Error(\%) & FLOPs & Pruned \\
\hline ResNet-20 [11] & 8.75 & $8.16 \mathrm{E}+07$ & - \\
ResNet-20-pruned-70 & 8.87 & $5.71 \mathrm{E}+07$ & $30 \%$ \\
ResNet-20-pruned-50 & 10.98 & $4.08 \mathrm{E}+07$ & $50 \%$ \\
ResNet-20-pruned-30 & 14.67 & $2.45 \mathrm{E}+07$ & $70 \%$ \\
\hline \hline ResNet-32 [11] & 7.51 & $1.38 \mathrm{E}+08$ & - \\
ResNet-32-pruned-70 & 7.53 & $9.68 \mathrm{E}+07$ & $30 \%$ \\
ResNet-32-pruned-50 & 10.75 & $6.91 \mathrm{E}+07$ & $50 \%$ \\
ResNet-32-pruned-30 & 13.73 & $4.15 \mathrm{E}+07$ & $70 \%$ \\
\hline
\end{tabular}

Similar to VGG-16, we iteratively pruned ResNet-20/32 from the first block to the last. In the batch normalization layer, the channels corresponding to the pruned filters were also pruned. Within pruning iterations, we estimated new kernels using our proposed method and then applied final fine-tuning with a fixed learning rate of $10^{-3}$, which was performed at the last round. A horizontal flip was applied for data augmentation. We pruned both models using three different compression rates, pruned-70, pruned-50, and pruned-30, where 70\%, 50\%, $30 \%$ of filters were preserved respectively in each block. Due to reduced redundancy and the more compact nature of the ResNet architecture, pruning a large number of filters is more challenging and affects the overall accuracy. However, we were able to prune $30 \%$ of both models with only $0.12 \%$ accuracy decrease on ResNet- 20 and $0.02 \%$ accuracy decrease on ResNet-32. The results are presented in Table 6.3.

\subsubsection{ResNet-20/ResNet-32 on CUB-200}

We also evaluated the performance of the proposed method on larger data: the CUB-200 dataset on ResNet architecture. Our focus was to reduce the number of convolutional channels in each filer and the approximated floating-point operations (FLOPs). In the first experiment, we began with an ImageNet pre-trained model to fine-tune the ResNet models, as fine-tuning is a common approach adopted in many recognition tasks, and the CUB-200 examples are not 
large enough to train such models from scratch; it seemed that the models overfit the training data and had poor generalization performance. In our implementation, a horizontal flip was applied for data augmentation. The Adam optimizer [63] was used, where each batch contains 32 randomly shuffled images. For our experiment, we started with a learning rate of 0.001 , a fixed momentum of 0.9 , and a fixed weight decay of 0.0005 . The learning rate was scheduled to be reduced after every 40 epochs.

We used our proposed method to prune unimportant filters of both ResNet-20 and ResNet32 and convert a large model into a smaller one with a minor drop in model accuracy. Similar to CIFAR-10, we pruned both ResNet-20 and ResNet-32 models on CUB-200 using three different compression rates, pruned-70, pruned-50, and pruned-30, where 70\%,50\%, 30\% of filters are preserved in each block respectively. The results are shown in Table 6.4. Due to the small number of training examples, the accuracy of the pruning model ultimately could not be improved, and the final fine-tuning attains a limited contribution to completely recovering the accuracy of the reference model. Another issue is that the ResNet architecture has little redundancy, so pruning a large number of filters is more challenging and affects the overall accuracy.

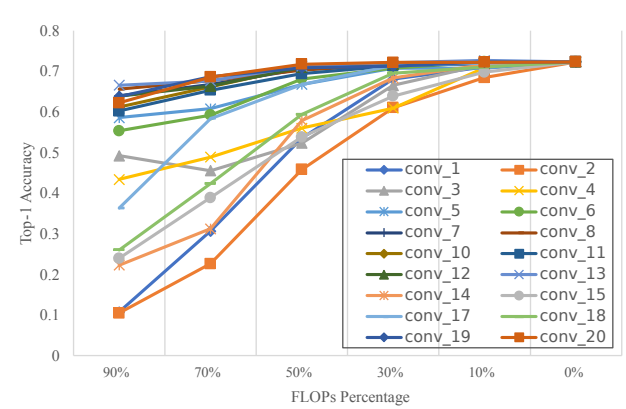

A. ResNet-20

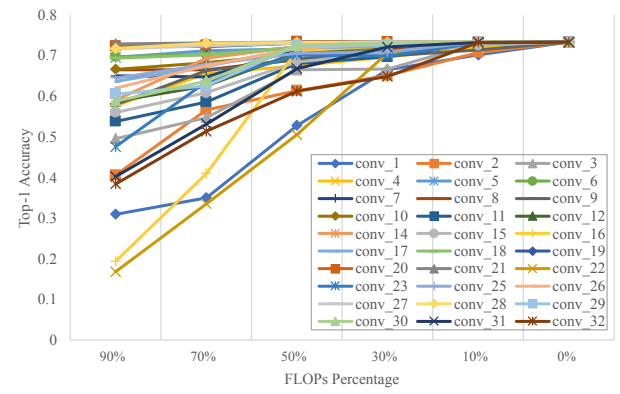

B. ResNet-32

Figure 6.10: Layer-wise pruning of ResNet 20/32 on CUB-200.

Fig. 6.10 shows the classification accuracy of both ResNet-20 and ResNet-32 on CUB200 after pruning each layer using our proposed method. Despite the compactness and reduced redundancy of ResNet models, our pruning method was able to compute a measure of relevance that identifies the less critical filters and prunes them accordingly, as illustrated in Fig. 6.10. In other words, our pruning method removes the unimportant part which does not appreciably contribute much to the final model performance. Fig. 6.10 also shows that many layers were minimally affected by the pruning of their unnecessary parts, especially when using a low 
Table 6.4: Performance of pruning ResNet-20/32 on CUB-200 using different pruning rates. The classification error is reported.

\begin{tabular}{|l|l|l|l|}
\hline Model & Error(\%) & FLOPs & Pruned \\
\hline ResNet-20 [11] & 27.67 & $2.95 E+08$ & - \\
ResNet-20-pruned-70 & 29.69 & $2.07 E+08$ & $30 \%$ \\
ResNet-20-pruned-50 & 32.95 & $1.48 E+08$ & $50 \%$ \\
ResNet-20-pruned-30 & 39.99 & $8.86 E+07$ & $70 \%$ \\
\hline \hline ResNet-32 [11] & 26.68 & $1.82 E+09$ & - \\
ResNet-32-pruned-70 & 29.08 & $1.27 E+09$ & $30 \%$ \\
ResNet-32-pruned-50 & 35.48 & $9.08 E+08$ & $50 \%$ \\
ResNet-32-pruned-30 & 41.63 & $5.45 E+08$ & $70 \%$ \\
\hline
\end{tabular}

compression rate. This also explains why the overall accuracy was not recovered completely when a small set of training examples was used for the final fine-tuning.

\subsubsection{ResNet-50 on ImageNet}

To thoroughly validate our proposed method, we also evaluated its performance on a large-scale dataset: the ImageNet data [279] with ResNet-50 [11]. In the initial experiment, we started with a pre-trained model in Keras Applications ${ }^{2}$, which achieved classification errors of $25.1 \%$ in top-1 error and $7.9 \%$ in top-5. The classification errors are reported on the standard validation set, using the single central crop. The resized images are center-cropped to $224 \times 224$. To prune the ResNet-50, we followed the setting of ThiNet [207], where the first two layers of each residual block are pruned; this leaves the output block and the projection shortcuts consistent. The entire network was pruned from block $2 \mathrm{a}$ to $5 \mathrm{c}$ iteratively. The corresponding channels in the batch normalization layer were also pruned. Within pruning iterations, new kernels were estimated using the proposed method. After pruning, a final fine-tuning was performed for 20 epochs at the last round. The model was pruned using three different compression rates, pruned-70, pruned-50, and pruned-30, where $70 \%, 50 \%, 30 \%$ of filters were preserved respectively in each targeted block. We were able to prune $30 \%$ of ResNet-50 with $0.24 \%$ reduction in the original model's top- 1 error and with only a $0.03 \%$ drop in the top- 5 error. The results are presented in Table 6.5, showing that significant performance degradation arises with an increased pruning rate. A much smaller model can be obtained at the cost of further accuracy reduction.

\footnotetext{
${ }^{2}$ https://keras.io/api/applications/
} 
Table 6.5: Performance of pruning ResNet-50 on ImageNet using different pruning rates. The classification errors (Top-1/5 Err.) are reported on the standard validation set, using the single central crop.

\begin{tabular}{|l|l|l|l|l|}
\hline Model & $\begin{array}{l}\text { Top-1 } \\
\text { Err.(\%) }\end{array}$ & $\begin{array}{l}\text { Top-5 } \\
\text { Err.(\%) }\end{array}$ & \#FLOPs & Pruned \\
\hline \hline ResNet-50 [11] & 25.10 & 7.90 & $7.72 \mathrm{E}+09$ & - \\
ResNet-50-pruned-70 & 25.34 & 7.93 & $4.88 \mathrm{E}+09$ & $30 \%$ \\
ResNet-50-pruned-50 & 26.41 & 8.17 & $3.41 \mathrm{E}+09$ & $50 \%$ \\
ResNet-50-pruned-30 & 30.95 & 10.99 & $2.20 \mathrm{E}+09$ & $70 \%$ \\
\hline
\end{tabular}

We compared our proposed approach with other state-of-the-art pruning methods. Table 6.6 presents the comparison results on ResNet-50 and ImageNet. For a fair comparison, all compared methods targeted the first and second layers of each residual block and adopted the same compression ratio of 0.7 and 0.5 , where $70 \%$ and $50 \%$ of filters were preserved in each targeted layer respectively. In the first stage, we compared our proposed approach with other filter-level pruning methods including ThinNet [207], SSR-L2 [282], Weights Sum [228], and APoZ [232]. These methods evaluate the importance of intermediate units and prune them accordingly. Because the pruning pipeline of these filter-level pruning methods is the same, it is a fair comparison, and our proposed approach has achieved better results. In top-1 error, the proposed approach surpasses the baseline methods ThinNet, SSR-L2, Weights Sum, and APoZ by $1.96 \%, 2.34 \%, 3 \%$ and $2.94 \%$ respectively, representing significant improvements on the ImageNet with a compression ratio of 0.5 . In top-5 error, our method also outperforms ThinNet, SSR-L2, Weights Sum, and APoZ by $0.94 \%, 1.84 \%, 2.15 \%$ and $2.14 \%$ respectively, with the same compression ratio. The relationship between a semantic concept and individual hidden unit representations is directly considered in our proposed approach, which can adaptively determine the function of individual CNN filters to deliver essential information and prune the lower impact filters on the global output.

We also compared our proposed approach with three training-based pruning methods including AutoPruner [239], C-SGD [234], and DCP [283]. The results are summarized in Table 6.6. The pruning procedure of these methods is considered a single end-to-end trainable system, where evaluating channels' importance, pruning channels, and fine-tuning are performed jointly during an iterative training procedure. Although they achieve remarkable accuracy, their computational costs and memory requirements are increased as modern GPUs do not benefit from sparse convolutions. Pruning procedures based on iterative training often change the optimization function and even introduce many hyper-parameters, making the training more challenging. However, our pruned networks show a similar reduction in FLOPs 
with comparable accuracy. Note that we adopt the same compression ratios and target the same layer for ResNet-50. When comparing with AutoPruner [239], our method achieves a 0.08\% increase in the top- 1 error and $0.45 \%$ increase in the top-5 error with similar FLOPs. Compared to the iteratively optimized pruning methods, our approach has several advantages. It is capable of pruning any CNN using a single forward pass without the need for a training process or back-propagation. For the forward pass, we only select a few images from each category to form our evaluation set used to find the optimal channel subset. Consequently, the small number of instances are used to find the optimal filter subset via Algorithm 4. After pruning all layers, we only fine-tune the pruned models once for a reasonable number of epochs to reduce training time.

Table 6.6: Comparison among several state-of-the-art pruning methods on ResNet-50 and ImageNet. The Acc. $\downarrow$ (\%) denotes the accuracy drop between the baseline model and the pruned model.

\begin{tabular}{|l|l|l|l|l|l|}
\hline Model & $\begin{array}{l}\text { Top-1 } \\
\text { Acc. } \downarrow \text { (\%) }\end{array}$ & $\begin{array}{l}\text { Top-5 } \\
\text { Acc. } \downarrow \text { (\%) }\end{array}$ & \#Param. & \#FLOPs & Pruned \\
\hline \hline C-SGD-70 [234] & $\mathbf{0 . 0 6}$ & 0.10 & $16.94 \mathrm{E}+06$ & $4.88 \mathrm{E}+09$ & $30 \%$ \\
C-SGD-50 [234] & $\mathbf{0 . 7 9}$ & 0.47 & $12.38 \mathrm{E}+06$ & $3.41 \mathrm{E}+09$ & $50 \%$ \\
DCP [283] & 1.06 & 0.61 & $12.38 \mathrm{E}+06$ & $3.41 \mathrm{E}+09$ & $50 \%$ \\
AutoPruner [239] & 1.39 & 0.72 & $12.38 \mathrm{E}+06$ & $3.41 \mathrm{E}+09$ & $50 \%$ \\
\hline \hline ThinNet-70 [207] & 1.27 & 0.09 & $16.94 \mathrm{E}+06$ & $4.88 \mathrm{E}+09$ & $30 \%$ \\
ThinNet-50 [207] & 3.27 & 1.21 & $12.38 \mathrm{E}+06$ & $3.41 \mathrm{E}+09$ & $50 \%$ \\
SSR-L2 [282] & 3.65 & 2.11 & $12.38 \mathrm{E}+06$ & $3.41 \mathrm{E}+09$ & $50 \%$ \\
Weights Sum [228] & 4.31 & 2.42 & $12.38 \mathrm{E}+06$ & $3.41 \mathrm{E}+09$ & $50 \%$ \\
APoZ [232] & 4.25 & 2.41 & $12.38 \mathrm{E}+06$ & $3.41 \mathrm{E}+09$ & $50 \%$ \\
\hline \hline ResNet-50-pruned-70 & 0.24 & $\mathbf{0 . 0 3}$ & $16.94 \mathrm{E}+06$ & $4.88 \mathrm{E}+09$ & $30 \%$ \\
ResNet-50-pruned-50 & 1.31 & $\mathbf{0 . 2 7}$ & $12.38 \mathrm{E}+06$ & $3.41 \mathrm{E}+09$ & $50 \%$ \\
\hline
\end{tabular}

\subsubsection{Feature Map Visualization}

We carried out a visual assessment to provide a convincing analysis of the motivation to fundamentally rely on evaluating the alignment between a semantic concept and individual hidden unit representations. Fig. 6.11 shows different examples from the ImageNet dataset with their semantic segmentation and visualization of feature maps binary segmentation for the first layer of the ResNet-50 first block (i.e. res $2 a)$. The channels with red borders correspond to the channels selected to be eliminated in our pruned model when the compression rate is set to $30 \%$. Fig. 6.11 demonstrates that the selected channels are less informative (i.e. channels with title 2, $6,23,31,35$ and 56) compared to other channels that highly correlated to the region of an object class across different images. For instance, the channel's visualization with title 62 reveals 

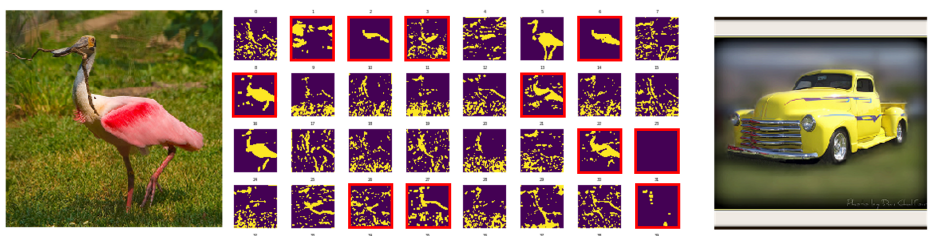

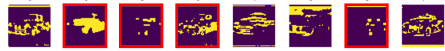

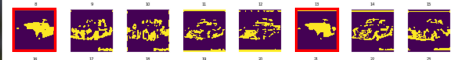

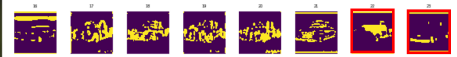

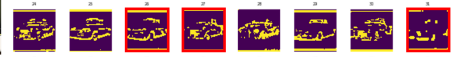

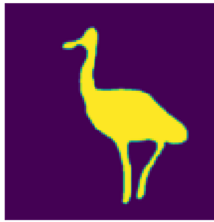
(3)

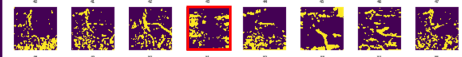

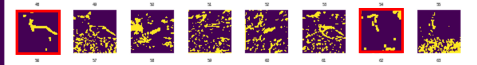

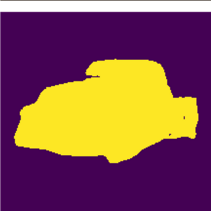
서에

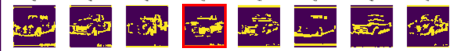

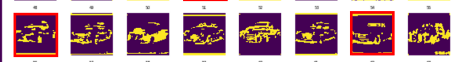

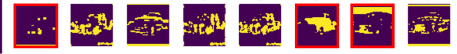
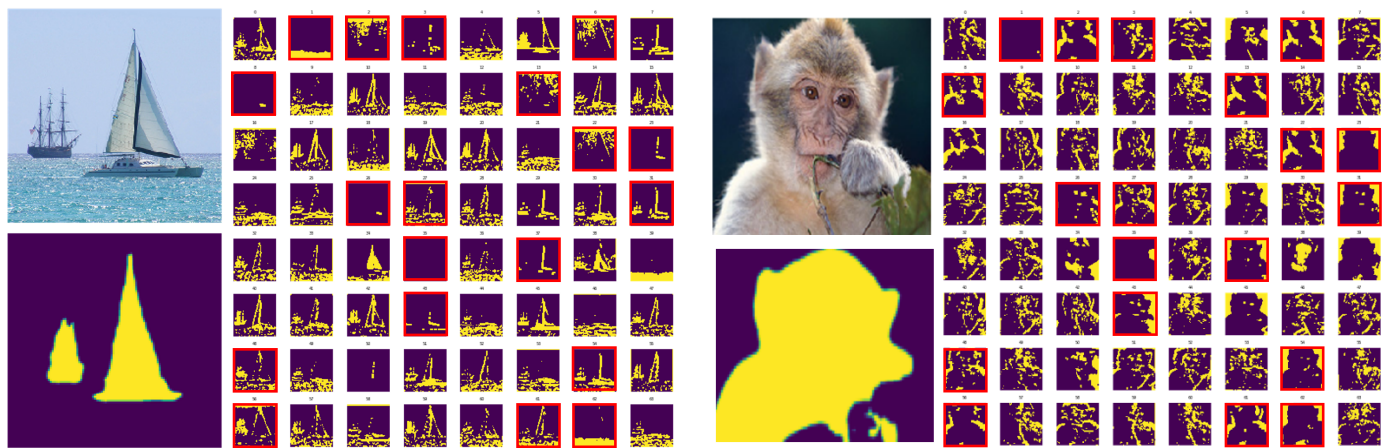

Figure 6.11: Four different input images of ImageNet dataset and their semantic segmentation and visualization of feature maps binary segmentation of ResNet-50 first block (i.e. res2a). Feature maps with red borders are the eliminated channels in our pruned model (this figure is best viewed in color).

that this particular feature map focuses on background rather than foreground objects. This demonstrates that our proposed method determines individual CNN filters' function to deliver essential information with strong discriminative power for the model. It can also be observed that non-pruned channels are related to the concept of an object, which closely matches the semantic segmentation of an object (e.g., pickup truck, spoonbill, and schooner). The apparent commonality among these channels is that representations are object classes appropriate with diverse visual appearances. Another remarkable appearance is that many channels represent parts of the object.

\subsubsection{Comparison with Filter Selection Criteria}

\subsubsection{Implementation Details}

Classification performance was used in order to evaluate the impact of our filter selection criteria. An ablation study provides a scheme to evaluate the effectiveness of measuring filters' 
importance quantitatively. This procedure typically refers to the removal of some parts of the model and the study of its performance, as crucial filters capture meaningful information and contribute substantially to the model's final performance. We ablated non-informative filters by forcing their activation to be zero and computed the classification accuracy on the test set. Quantifying the influence of the ablation on the classification performance allows for an impartial evaluation, in order to distinguish the essential filters in a CNNs and measure their importance, allowing for layer-wise comparison. This method not only enables the evaluation of filters' importance, but can also detect the unimportant, redundant filters which can be safely pruned.

\subsubsection{Different Filter Selection Criteria}

Several criteria to estimate the importance of a feature map or convolutional kernel in the CNNs have been developed. To evaluate the effectiveness of our evaluation criterion, we compared our filter selection method with several baseline methods. These can be briefly summarized as follows:

- Random. Filters are randomly ablated.

- Weights sum [228]. Filters $(i)$ with lowest absolute weights sum values are ablated: $\psi_{i}=\sum_{i}|\omega(i,:,:,:)|$.

- Activation Mean [228]. $\psi_{i}=\frac{1}{N} \sum \operatorname{mean}(\tau(i,:,:))$, where $\tau$ is the activation values for filter $i$, and $N$ denotes the size of data. The feature maps with weak patterns and their corresponding filters and kernels are ablated.

- Mean gradient [229]. $\psi_{i}=\frac{1}{N} \sum$ mean $(\kappa(i,:,:))$, where $\kappa$ is the calculated gradient for each filter channel $i$, and $N$ denotes the size of data.

- LRP (Layer-wise Relevance Propagation) [233]. The LRP of each channel $i$ is calculated as its importance score $\psi_{i}=\frac{1}{N} \sum \sum(\operatorname{LRP}(i,:,:))$, where $N$ denotes the size of data; LRP calculates the summed relevance quantity of each channel in the network to the overall classification score, decomposing a classification decision into contributions for each channels.

All these baseline methods consider the higher scores as more important, which is motivated by the intuition that unimportant activation and filters have no influential outputs to the final prediction of a model. 


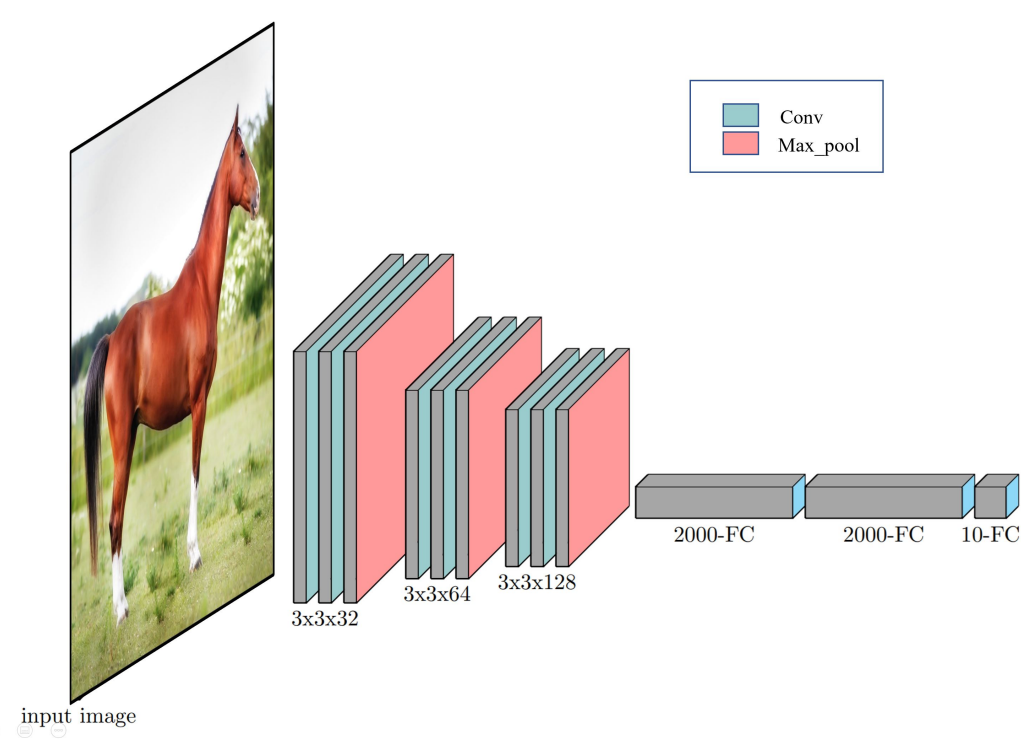

Figure 6.12: The architecture of the CNN model.

\subsubsection{Overall Performance Comparison}

Table 6.7 and Table 6.8 summarize the comparison results for two network architectures: our proposed CNN architecture and VGG-16 on CIFAR-10 with different pruning criteria. Our CNN architecture consists of three convolutional blocks, where each block has two convolutional layers with a filter size of $3 \times 3$ with 32 kernels in the first block, 64 kernels in the second block, and 128 kernels in the third block. Each block ends with a max-pooling layer. This is followed by three fully-connected layers consisting of 2,000, 2,000, and 10 neurons respectively. A standard Relu activation function was utilized. The detailed architecture of the CNN model is presented in Fig. 6.12. Using the ablation approach, the importance of the filters was evaluated by employing different selection criteria in a fully trained model. We compared our method with such baselines, and the results are reported in Fig. 6.13, where different compression rates are used. Table 6.7 and Table 6.8 also show different methods to measure the importance of filters, using fixed compression ratio $=0.5$, where $50 \%$ of channels are preserved after pruning. The tables show layer-wise results for each layer, where we ablated layer-bylayer and calculated the accuracy for each layer separately. For random selection criteria, the mean value of three runs are reported.

Our ablation study has shown that for both architectures, MV achieves higher classification performance when compared with other baselines. This demonstrates the robustness of our proposed method in identifying the essential filters. With VGG16, as shown in Table 6.7, our 


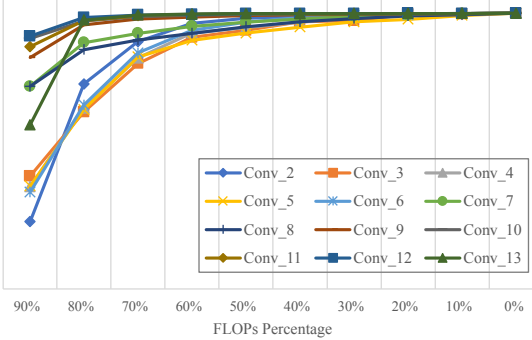

A. Proposed Method

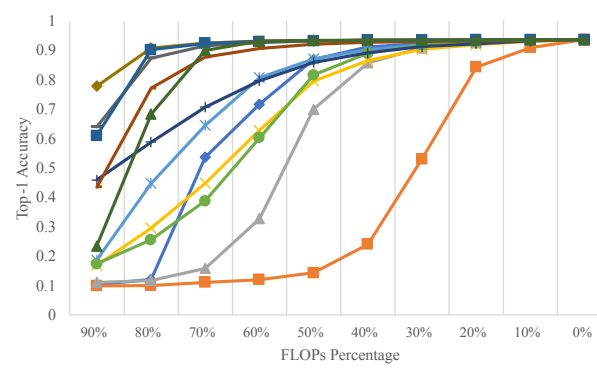

C. Mean-Mean [228]

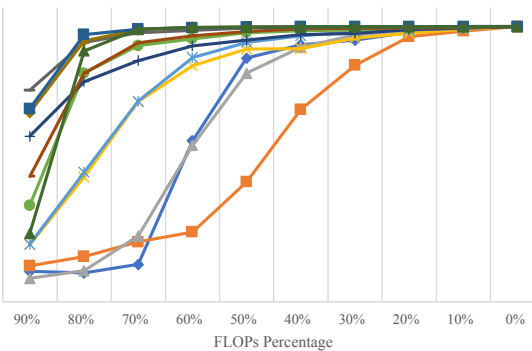

E. Mean Gradient [229]

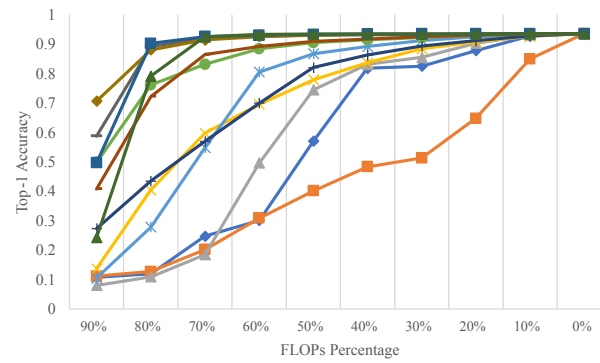

B. Random

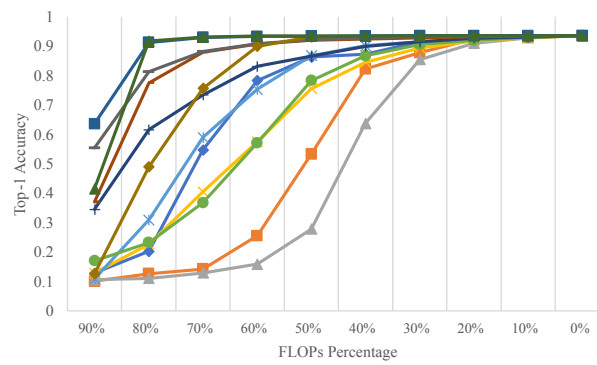

D. Weights Sum [228]

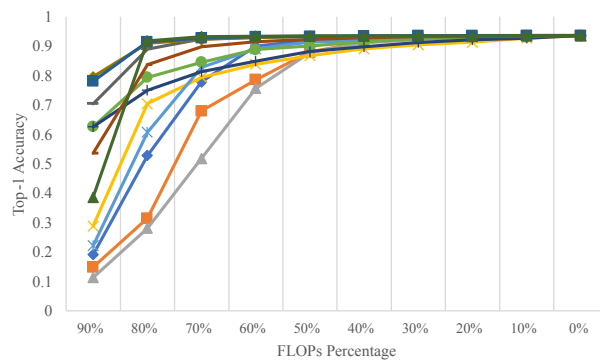

F. LRP [233]

Figure 6.13: Comparison of layer-wise pruning methods for VGG-16 on CIFAR-10. The test accuracy is reported after ablating the unimportant filters with different compression ratios

pruning method achieved the best results when using a compression ratio of 0.5 for each layer of the reference model. Fig. 6.13A. demonstrates that the performance of the pruned model with our proposed method is relatively consistent, as model FLOPs reduce, especially when reaching a reduction of $60 \%$. Our method delivers the best result among all baselines. On the other hand, it has less impact on the dropping of model accuracy while reducing FLOPs compared with the LRP-based method, which is also inspired by neural network interpretability. These results indicate an interesting potential research direction of combining the two fields of 
Table 6.7: Overall results of layer-wise pruning utilizing different filter selection criteria. The test accuracy is reported after ablating the unimportant filters. The results were conducted on an NVIDIA GeForce GTX 1080 GPU to prune the VGG-16 model on CIFAR-10 with a compression ratio of 0.5 , where $50 \%$ of filters were preserved after pruning. For conv_2, the running time of identifying important filters is also reported (the model's forward time is approximately $9 \mathrm{~s}$ ).

\begin{tabular}{|r|l|c|c|c|c|c|c|c|c|c|c|c|}
\hline & Conv_2 & Conv_3 & Conv_4 & Conv_5 & Conv_6 & Conv_7 & Conv_8 & Conv_9 & Conv_10 & Conv_11 & Conv_12 & Conv_13 \\
\hline Random & $0.8290(0.073 \mathrm{~ms})$ & 0.408 & 0.7776 & 0.8592 & 0.8824 & 0.895 & 0.8605 & 0.9194 & 0.9307 & 0.931 & 0.932 & 0.9341 \\
Weights-sum [228] & $0.8630(2.015 \mathrm{~ms})$ & 0.5333 & 0.2785 & 0.7558 & 0.8681 & 0.7834 & 0.8655 & 0.92 & 0.9218 & 0.9321 & 0.934 & 0.9343 \\
Mean-mean [228] & $0.8686(4.5 \mathrm{~s})$ & 0.1433 & 0.6995 & 0.795 & 0.8707 & 0.8157 & 0.8587 & 0.9198 & 0.9323 & 0.9314 & 0.932 & 0.9339 \\
Mean Gradient [229] & $0.5706(11.1 \mathrm{~s})$ & 0.4017 & 0.7448 & 0.7795 & 0.868 & 0.9052 & 0.821 & 0.9095 & 0.9313 & 0.9313 & 0.9331 & 0.9345 \\
LRP [233] & $0.9186(13.2 \mathrm{~s})$ & 0.88 & 0.8698 & 0.8675 & 0.9067 & 0.9005 & 0.8815 & 0.9234 & 0.9316 & 0.9328 & 0.9332 & 0.9342 \\
Our method & $\mathbf{0 . 9 1 9 9}(10.9 \mathrm{~s})$ & $\mathbf{0 . 8 6 4}$ & $\mathbf{0 . 8 7 8 4}$ & $\mathbf{0 . 8 6 8 6}$ & $\mathbf{0 . 9 1 5}$ & $\mathbf{0 . 9 0 5 9}$ & $\mathbf{0 . 8 9 0 7}$ & $\mathbf{0 . 9 2 8 6}$ & $\mathbf{0 . 9 3 2}$ & $\mathbf{0 . 9 3 4 7}$ & $\mathbf{0 . 9 3 4 6}$ & $\mathbf{0 . 9 3 4 9}$ \\
\hline
\end{tabular}

Table 6.8: Overall results of layer-wise pruning utilizing different filter selection criteria. The results are reported on our small CNN model on CIFAR-10 with a compression ratio of 0.5 , where $50 \%$ of filters were preserved after pruning. The test accuracy is reported after ablating the unimportant filters.

\begin{tabular}{|r|c|c|c|c|c|}
\hline & Conv_2 & Conv_3 & Conv_4 & Conv_5 & Conv_6 \\
\hline Random & 0.7935 & 0.3556 & 0.6843 & 0.7096 & 0.7975 \\
Weights-sum [228] & 0.8027 & 0.4500 & 0.7091 & 0.6852 & 0.7645 \\
Mean-mean [228] & 0.7790 & 0.3761 & 0.7465 & 0.7133 & 0.7855 \\
Mean Gradient [229] & 0.8471 & 0.2673 & 0.6149 & 0.6214 & 0.7437 \\
LRP [233] & 0.8475 & 0.6122 & 0.7729 & 0.7466 & 0.8091 \\
Our method (Sum-IoU) & 0.8422 & 0.6709 & 0.7515 & 0.6988 & 0.8152 \\
Our method (MV) & $\mathbf{0 . 8 5 9 9}$ & $\mathbf{0 . 6 9 3 5}$ & $\mathbf{0 . 7 8 5 8}$ & $\mathbf{0 . 7 2 0 1}$ & $\mathbf{0 . 8 2 3 9}$ \\
\hline
\end{tabular}

interpretability and model compression research.

Interestingly, ablating filters with random selection showed that the first few layers had stronger negative effects and more synergistic filters compared with higher hidden layers. It was also observed that the higher hidden layers were significantly redundant and more classspecific. This observation is consistent with a previous theoretical proposal by [275]. One reasonable explanation is that the neural networks hierarchically learn representations. Hence, the first layers are not relevant to a specific object. Still, they build feature representations of all input images that are joined to form more relevant object features in the later layers. By ablating these fundamental features, deeper layers fail to produce class-specific features and have a more negative impact on overall accuracy.

Although random selection is neither robust nor applicable in practice [207], it offers insight and demonstrates that the detection of principal filters is a critical approach when pruning redundant filters. The experiment empirically confirms that our importance method is sufficient, given that ablating filters with low values in the layers had a negligible impact on the overall accuracy compared to all baselines. As shown in Table 6.7 and Table 6.8, the experimental results for both networks show that the method substantially outperformed the baselines. Our proposed method to measure filters' importance helps not only to remove redundant 
nodes and compress the neuron network, but also to understand their inter-relationships and how said filters impact the model. The experiment confirms that selecting the right criteria to evaluate filters' importance throughout all layers can guarantee a successful pruning approach. In Table 6.7, we reported the running time of different filter selection approaches. The running speed of data-driven methods relies on model inference speed and dataset size. On an NVIDIA GeForce GTX 1080 GPU, it takes 9.186s to apply forward passing through the optimized VGG-16 model on CIFAR-10. Our proposed approach takes $10.9 \mathrm{~s}$ to identify the important filters, which is faster than some competitive methods. For ResNet-50 on ImageNet, the time cost to estimate IoU scores and MV values is 422.3 s of ResNet-50 first block (i.e. res2a).

\subsubsection{Comparison of Kernal Estimation (KE) vs. Fine-Tuning (FT)}

In order to gather conclusive evidence to evaluate the effectiveness of our kernels estimation (KE) method, an experiment based on the iterative layer-wise pruning process was carried out using the VGG-16 model on CIFAR-10. Thus, we were able to fairly compare our KE method with the standard fine-tuning (FT) procedure that is performed to preserve the original accuracy or recover the damage that might occur during the compression phase. After pruning each layer with a fixed compression ratio of 0.5 , our KE method, as well as the FT procedure, were applied to improve the performance degradation. The experiments were performed on the training set using three different numbers of trained examples, i.e. 200, 500, and 1,000. We estimated new kernels and performed the fine-tuning using these settings with the same amount of iteration. The comparative results are shown in Table 6.9, which demonstrates that with a small number of examples, KE performed much better with less run-time requirements. The approximate time needed to complete the process of each method is shown on the table. The KE achieved higher classification performance, especially when the whole network was cumulatively pruned. Our experiment has shown that both KE and FT improved the accuracy after pruning each layer. These results demonstrate the necessity of adopting such steps to recover model accuracy which has been damaged iteratively. However, even though the strategy of iterative pruning with fine-tuning is the typical setting for CNN pruning, it incurs expensive computational costs, significant inference time, and high storage requirements. Such an iterative process requires significant inference costs, including costs related to the creation of a new model, loading of parameters, and retraining of the whole model. 
Table 6.9: Comparison of layer-by-layer pruning with fine-tuning (FT) and kernels estimation (KE) using the VGG-16 model on CIFAR-10 using 200, 500, and 1000 training examples. These results were conducted on NVIDIA GeForce GTX 1080 GPU. The pruning is applied in a layer-by-layer fashion from the shallow layers to the deeper ones sequentially. The test accuracy is reported before and after independently performing fine-tuning and kernels estimation. For conv13, the running time of both procedures is also reported.

\begin{tabular}{|c|c|c|c|c|c|c|c|c|c|c|c|c|}
\hline & Conv_2 & Conv_3 & Conv_4 & Conv_5 & Conv_6 & Conv_7 & Conv_8 & Conv_9 & Conv_10 & Conv_11 & Conv_12 & Conv_13 \\
\hline \multicolumn{13}{|c|}{200 samples } \\
\hline Before KE & 0.9173 & 0.8536 & 0.8151 & 0.8084 & 0.8482 & 0.8219 & 0.7546 & 0.7988 & 0.7839 & 0.7769 & 0.8028 & 0.7707 \\
\hline After KE & 0.9338 & 0.9265 & 0.9195 & 0.8923 & 0.8746 & 0.8619 & 0.8151 & 0.8028 & 0.7971 & 0.7958 & 0.7954 & $\mathbf{0 . 7 9 4 1}(5.86 \mathrm{~s})$ \\
\hline Before FT & 0.9173 & 0.8254 & 0.7362 & 0.705 & 0.6991 & 0.671 & 0.5714 & 0.6128 & 0.6163 & 0.5881 & 0.6001 & 0.6091 \\
\hline After FT & 0.9203 & 0.8873 & 0.8499 & 0.7681 & 0.7401 & 0.6926 & 0.632 & 0.6317 & 0.6148 & 0.6034 & 0.6074 & $0.6044(22.60 \mathrm{~s})$ \\
\hline \multicolumn{13}{|c|}{500 samples } \\
\hline Before KE & 0.9173 & 0.8498 & 0.8232 & 0.82 & 0.8494 & 0.8279 & 0.7699 & 0.8077 & 0.8055 & 0.806 & 0.8171 & 0.7979 \\
\hline After KE & 0.9332 & 0.9271 & 0.9196 & 0.8942 & 0.8771 & 0.8647 & 0.829 & 0.8195 & 0.8157 & 0.8161 & 0.8145 & $\mathbf{0 . 8 1 3 8}$ (6.81s) \\
\hline Before FT & 0.9173 & 0.826 & 0.7395 & 0.7143 & 0.7102 & 0.7026 & 0.6341 & 0.6759 & 0.6765 & 0.6663 & 0.6609 & 0.6751 \\
\hline After FT & 0.9239 & 0.8918 & 0.8559 & 0.7859 & 0.7647 & 0.7326 & 0.6817 & 0.6848 & 0.6808 & 0.6699 & 0.6725 & $0.6723(22.74 \mathrm{~s})$ \\
\hline \multicolumn{13}{|c|}{1000 samples } \\
\hline Before KE & 0.9173 & 0.851 & 0.8165 & 0.8203 & 0.8556 & 0.8369 & 0.7935 & 0.8177 & 0.8127 & 0.8147 & 0.8238 & 0.809 \\
\hline After KE & 0.9331 & 0.9275 & 0.9202 & 0.8958 & 0.8811 & 0.8685 & 0.8404 & 0.8303 & 0.8247 & 0.8245 & 0.8243 & $\mathbf{0 . 8 2 2 7}$ (8.81s) \\
\hline Before FT & 0.9173 & 0.8325 & 0.7507 & 0.7173 & 0.7499 & 0.7247 & 0.6506 & 0.6939 & 0.6963 & 0.6858 & 0.6888 & 0.6887 \\
\hline After FT & 0.9235 & 0.8969 & 0.8622 & 0.8007 & 0.7777 & 0.7472 & 0.7011 & 0.709 & 0.6952 & 0.6915 & 0.6904 & $0.6948(23.34 \mathrm{~s})$ \\
\hline
\end{tabular}

\subsection{Summary}

In this chapter, we have proposed a novel framework based on an effective channel-level pruning method, considering the power of novel neural network interpretability in evaluating the importance of feature maps. Based on the discriminative ability of interpretable latent representations, a majority voting technique is proposed to compare the degree of alignment values among filters and assign a voting score to quantitatively evaluate the importance of feature maps. The experimental results show the effectiveness of our filter selection criteria, which outperforms all other pruning criteria. It also allows for the identification of layers which are robust or sensitive to pruning, and this can be beneficial for further improving and understanding the architectures. We also propose a simple yet effective method to estimate new convolution kernels based on the remaining, crucial channels to accomplish effective CNN compression. The experimental results on CIFAR-10, CUB-200, and ImageNet (ILSVRC 2012) datasets demonstrate the effectiveness of our pruning framework in maintaining or even improving accuracy after removing unimportant filters. Our results also display the excellent performance of our proposed method. Moreover, our pruned model can be further pruned into even smaller models by adopting any existing model compression method. Our potential future work is to extend this framework and combine it with other pruning criteria to deeply explore the problem of CNN pruning from an interpretable perspective, aiming to link model compression and interpretability research. 
In the following chapter, we present a comprehensive review of time-series data analysis, introducing the use of DeepCluster methodology to learn and cluster temporal features from accelerometer data for the clustering of animal behaviors. An evaluation and discussion are given on real-world data. We also identify state-of-the-art and provide an outlook on the field of deep time-series clustering. 


\section{Chapter 7}

\section{Deep Time-Series Clustering}

\section{Contents}

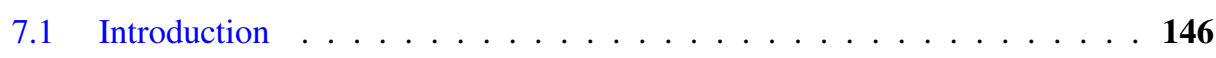

7.2 Time-series Data Type . . . . . . . . . . . . . . . . . . . . . 147

$7.2 .1 \quad$ Univariate . . . . . . . . . . . . . . . . . . . . . . . . . 147

7.2 .2 Multivariate . . . . . . . . . . . . . . . . . 147

7.2 .3 Tensor Fields . . . . . . . . . . . . . . . . . . . . . . 148

$7.2 .4 \quad$ Multifield . . . . . . . . . . . . . . . . . . . . . . . . . . . 149

7.3 Conventional Time-series Analysis . . . . . . . . . . . . . . . . 149

7.3.1 Similarity Measures and Features Extraction . . . . . . . . . . 151

7.3.2 Conventional Clustering Algorithms . . . . . . . . . . . 155

7.4 DeepCluster Method Applied to Biological Time-series Data: A Case Study 157

7.4.1 Network Architectures for ICBD . . . . . . . . . . . . . . . 158

7.4.2 Imperial Cormorant Birds Dataset (ICBD) and Pre-processing . . 161

7.4.3 Experiment and Discussion . . . . . . . . . . . . . . 163

7.5 State-of-the-art and Outlook . . . . . . . . . . . . . . . 165

7.5.1 Different Network Architectures . . . . . . . . . . . . . . 165

7.5.2 Different Clustering Methods . . . . . . . . . . . . . . . 168

7.5.3 Deep Learning Heuristics . . . . . . . . . . . . . . . . . . . . . . 169

7.5.4 DTSC Applictions ． . . . . . . . . . . . . . . . . . . . 170

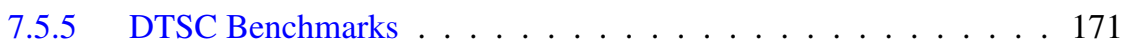

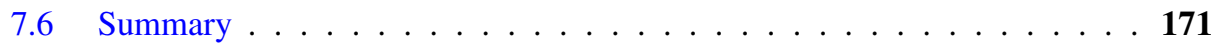




\subsection{Introduction}

Recent advances in time-series clustering have shown great success in a range of fields, including networks and systems, meteorology, social media, behavior analysis, trajectory data, biological science, and finance. Extracting useful structures from large volumes of data requires interdisciplinary research involving several domains such as statistics, machine learning, data visualization, pattern recognition, and high-performance computing [284]. Despite the progress made in time-series data clustering, the presence of noise, high dimensionality, and high feature correlation pose challenges in designing effective and efficient clustering algorithms. Traditional algorithms display limited performance with the increase in data dimensionality. Variants of deep learning methods have shown a robust ability in representation learning, finding the most success in supervised learning. Deep learning's ability to deal with high-level representations from data has inspired us to develop deep learning-based methods for clustering analysis. We have proposed the DeepCluster, a clustering approach embedded in a deep convolutional auto-encoder (DCAE), consisting of clustering and reconstruction objective functions. Its results on different datasets have shown the ability of deep clustering models to substantially outperform other methods in terms of clustering quality. We published this work in ICIP 2018 [5]. Moreover, we believe that we were the first to apply deep clustering methods to time-series data. Specifically, we modified the DCAE architectures to suit timeseries data; see section 7.4 for details. The work was done in 2017, and therefore we believe that we were the first to approach this topic and have made founding contributions to the area of deep clustering of time-series data; this chapter describes these contributions. Since 2018, several works have been reported on deep clustering of time-series data. We also review these works in this chapter and identify state-of-the-art and present an outlook on this important field of deep time-series clustering (DTSC).

The chapter is organised as follows. First, a detailed review of time-series data analysis is provided, focusing on a general classification for time-series data. All data types, as described in section 7.2, refer to the main definition of time-series data, and the section answers questions such as how time-series data are different, along with providing examples of this kind of data. Section 7.3 reviews the conventional time-series analysis with a discussion about similarity measures and feature extraction, which are important for time-series data as, usually, the quality of analysis techniques is significantly influenced by its selection. A comprehensive explanation for popular conventional clustering algorithms that have been used with time-series data is then offered. In section 7.4, we apply the DeepCluster method to real-world 
data; namely, the Imperial Cormorant bird dataset (ICBD) from the Biosciences department at Swansea University. Other recent works are subsequently discussed in section 7.5. The challenges of DTSC, opportunities, and future directions are also described. Finally, concluding remarks and summary are provided in section 7.6.

\subsection{Time-series Data Type}

Time-series data could be an umbrella term for many different data with an associated time component. It is defined as an ordered collection of observations or sequences of data points made over time, usually at uniform time intervals. In order to understand the complexity of time-series and explore the underlying processes, the processing and analysis of such data require particular supporting tasks and methods. Here, we classify time-series data into four categories, subsumed under the concepts of univariate, multivariate, tensor fields, and multifields. Hotz et al. [285] discuss the complex structure of scientific data and provide a clear definition of a multifield. Our four types, or categories, are generalized to include many related subtypes of time-series data in order to achieve a comprehensive classification for said data.

\subsubsection{Univariate}

The univariate time-series is a sequence that contains only one data value per temporal primitive $[69,284]$. It is a field of a single variable captured or observed through time. The temperature in a city spanning a period of time is a clear example of this type of data.

\subsubsection{Multivariate}

Multivariate time-series is a set of time-series which have the same timestamps [69,284]. This kind of time-series data is an array of variables or numbers at each point in time and can be a collection of multiple univariates captured through time, such as temperature and pressure readings, or associative multivariate, such as $3 \mathrm{D}$ acceleration measured from a tri-axial accelerometer, where each component of the multivariate has the same units and sensor source. As time-series data is an ordered collection of observations or a sequence of data points made over time, this special type of multivariate time-series data is relevant in many fields including biology, medicine, finance and animation. Multivariate time-series data have been used in manufacturing systems and predictive maintenance [286,287]. Time-series data obtained from gene expression measurement [288-290], for instance, can be used by biologists to understand 
the correlation between types of genes, analyze gene interactions, and compare regulatory behaviors for genes of interest. Medical experts also utilize time-series data from blood pressure measurements [291] to understand and deal with cases such as monitoring illness progression, and understanding ecological and behavioral processes related to a disease which may lead to improved diagnoses. Furthermore, time-series data such as that obtained from sampled transactions over a period of time [292-294], stock markets [124,295], and international financial markets [296,297] can be used in the financial field and are usually analyzed to understand and forecast market conditions. It is useful to find correlations between the data and test hypotheses about the market, as this helps in making correct decisions at the appropriate time under changing businesses and economic circumstances. A multivariate can also present timeseries data obtained from various data sets including metadata, e.g. patient records [298, 299], employment records [300,301], and social networks [302].

\subsubsection{Tensor Fields}

These comprise an array of data arranged on a regular grid with a variable number of axes [32]. They can be described as a quantity associated with each point in space-time as it has been extended to functions or distributions linked to points in space-time [285]. Dealing with spatiotemporal data, this type of time-series data is generalized to include many related subtypes: time-series of graphs and networks, time-series of spatial positions of moving objects, and time-series of spatial configurations/distributions.

\subsubsection{Time-series of Graph and Network}

Time-series data in the form of networks consist of associated attributes such as nodes and edges that reflect different kinds of behavior over time. Node or edge attributes of dynamic graphs can be introduced as time-series. This kind of time-series data helps with understanding different temporal patterns and evaluating the network dynamics in general [303-307]. As each machine (e.g. engines or computers) typically consists of a large number of sensors that produce massive quantities of data, time-series data can be obtained from the nodes of such machines over a period of time, such as CPU load, memory usage, network load, and data center chiller sensor, helping to improve the understanding of how machines are used in practice and analyze the performance and behaviors of such systems [308-314]. Indeed, analyzing this data can help users and experts understand and evaluate the network dynamics. 


\subsubsection{Time-series of Spatial Positions of Moving Objects}

Spatial positions of moving objects data with an associated time component classifies as trajectory data. It presents different places over time, providing a clear idea of spatio-temporal changes. The process and analysis of time-series data are important procedures for understanding the characteristics of the data and obtaining meaningful statistics which aid the exploration of the underlying processes, analysis, tracking, and representation of this type of data in order to understand and recognize the mobility of a diverse array of moving objects, such as vehicles [315-322], and aircrafts [317,318], which can lead to path discovery, movement analysis, and location prediction.

\subsubsection{Time-series of Spatial Configurations and Distributions}

Being able to extract useful insights from time-series of spatial distributions and configurations has become increasingly important due to significant growth in data science and rapid advancement in many technologies. In our research, we consider discovering behavioral patterns and finding interesting events that might take place in certain municipalities [323] and public or business sectors as spatial configurations and distributions. This identification of regular configurations and distributions over time is represented by a total number of events and behaviors extracted from a chosen spatial scale. Personal mobility behaviors and movement patterns [324-332], behaviors of animals [333,334], pattern changes in climate (weather) and the ozone layer [332,335-341], and behavior capture data made through time at often uniform time intervals $[135,342-346]$ can be regarded as instances of this type of data that take place in specific spatial identification.

\subsubsection{Multifield}

This kind of data, defined as a set of fields, provides enough flexibility to capture most types of compound datasets that occur in practice [285]. Combining multiple modality sensors such as gyroscopes, magnetometers and accelerometers with other environmental sensors is an example of this data type.

\subsection{Conventional Time-series Analysis}

For time-series data, the presence of noise, high dimensionality, and high feature correlation pose challenges for designing effective and efficient clustering algorithms compared to data 
without a temporal component $[284,347]$. Analyzing time-series data is nontrivial and can even vary over time due to complex interrelations between time-series variables. Xing et al. [348] describe three significant challenges for time-series analysis. First, many methods can only take input data as a vector of features. Unfortunately, there are no explicit features in sequence data. Second, feature selection is not easy because the dimensionality of the feature space can be high and computation can be costly. Third, since there are no explicit features in the raw data, building a partitioning task is burdensome in some applications. Therefore, efficiently handling the raw data in time-series is difficult without using similarity measures and feature extraction to reduce dimensionality and provide representative features of such data.

Computing the similarity between two data objects is considered one of the main differences between clustering of temporal and non-temporal data [155, 349]. The unique characteristics of time-series data such as noise, including outliers and shifts, and the varying length of time-series has made similarity measures one of the main challenges for clustering of time-series data [134]. When dealing with time-series data, the greatest challenge lies in replacing the distance/similarity measure for static data with a suitable one for time-series data, because it may be scaled and translated differently on both the temporal and behavioral dimensions $[347,350]$. Therefore, modifying distance functions to suit the characteristics of time-series data has become essential when developing a clustering method for time-series data. Petitjean et al. [351] introduced a kDBA method that combines Kmeans and dynamic time warping for better alignment. Moreover, Yang et al. [352] presented the K-Spectral Centroid (K-SC) method, using an invariant similarity metric to reveal the temporal dynamics. Lastly, Paparrizos et al. [353] developed a k-Shape method whereby the shapes of the timeseries are considered by applying cross-correlation measures. However, these methods are usually sensitive to noise and outliers because all time points are considered [354].

Furthermore, the quality of clustering methods is significantly affected by the choice of feature extraction technique. This fact made feature extraction very essential when clustering data. Guo et al. [355] proposed a feature-based approach to time-series clustering by applying independent component analysis to convert the raw time-series into a lower-dimensional feature vector and then further applying kmeans clustering on the extracted features. In addition, Zakaria et al. [356] employed u-shapelet algorithms to learn local patterns in a time-series, as they are highly predictive when performing clustering. Other recent advances in feature extractions have efficiently supported clustering tasks, where linear [357-360] and non-linear [361-364] methods have been adopted to transform the original time-series data into representative fea- 


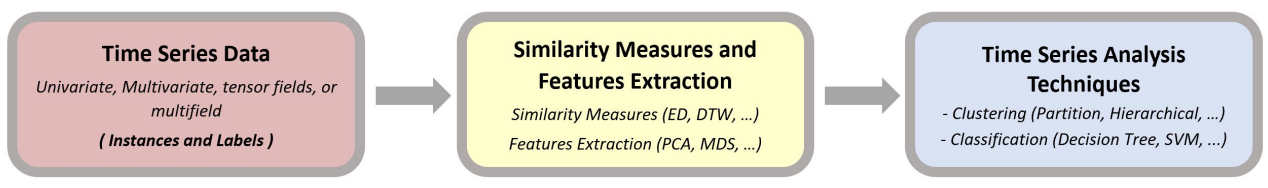

Figure 7.1: The pipeline of conventional time-series analysis.

tures, allowing unsupervised clustering methods to deal with beneficial features instead of raw data.

The conventional time-series analysis pipeline consists of three different perspectives, these being time-series data, similarity measures and feature extraction for time-series data, and timeseries clustering technique (see Fig. 7.1).

\subsubsection{Similarity Measures and Features Extraction}

Large time-series data require adequate pre-processing to gain an appropriate approximation of the underlying data representation. The aim is to generate a higher-level abstraction which represents the data while preserving the shape characteristics of the original data during dimensionality reduction. There are several dimensionality reduction techniques specifically designed for time-series which exploit the frequential content of the signal and its usual sparseness in the frequency space [365]. In general terms, choosing a distance measure is important and assists in dealing with outliers, amplitude differences, and time axis distortion. Furthermore, selecting important features in the data requires sufficient communication of knowledge from domain experts. Thus, the quality of clustering approaches is significantly affected by the choice of similarity measures and feature extraction techniques to obtain the relevant knowledge from the data. The below discussion about the types of methods is intended to provide a review of popular similarity measures and feature extraction techniques along with works which have been adopted in time-series data mining.

\subsubsection{Raw Data Similarity}

Most mining approaches utilize the concept of the similarity between a pair of time-series. Similarity measures must be chosen when dealing with time-series data in order to take into account outliers, different amplitude, and time axis distortion. When dealing with time-series 
data, efficiency and effectiveness are the main targets of representation methods and similarity measures [366]. Tornai et al. [367] argue that the distance between two sequences as a measurement plays an important role in the quality of clustering algorithms. The accuracy of such algorithms can be significantly impacted by the choice of similarity measures. Yahyaoui et al. [368] and Wang et al. [366] presented a comprehensive review of time-series measures, classifying them into four major categories: lock-step measures (e.g. Euclidean distance and Manhattan distance), elastic measures (e.g. longest common subsequence (LCS) and dynamic time warping (DTW)), pattern-based measures (e.g. spatial assembling distance (SpADe)), and threshold-based measures (e.g. threshold query based similarity search (TQuEST)). The types of methods, discussed below, are intended to provide a review of popular similarity measures.

Euclidean distance (ED): is a commonly used metric for time-series. It is defined between two time-series $\mathrm{X}$ and $\mathrm{Y}$ having length $\mathrm{L}$; therefore, the Euclidean distance, between each pair of corresponding points $\mathrm{X}$ and $\mathrm{Y}$, is the square root of the sum of the squared differences [369]. Thus, the two time-series being compared must have the same length, and the computational cost is linear in terms of temporal sequence length [370]. Along the horizontal axis, the distance between the two time-series is calculated by matching the corresponding points [371]. The Euclidean distance metric is very sensitive to distortion and noise [348], and is not able to handle one of the elements being compressed or stretched [334]. This approach is therefore not reliable, especially when computing similarity between time-series with different time durations [372].

Dynamic Time Warping (DTW): is another distance measure that is proposed to overcome some Euclidean distance limitations such as non-linear distortions. In DTW, the two time-series do not have to be the same length, and the idea is to align (warp) the series before computing the distance [348]. However, two temporal points with completely different local structures might be mistakenly matched by DTW. This issue can be addressed by improving the alignment algorithm, e.g. shape dynamic time warping. It considers point-wise local structural information [373].

Due to its quadratic time complexity, DTW does not scale well when dealing with large datasets. Despite this, it is widely used in various applications, such as in bioinformatics, finance and medicine [374]. DTW has several local constraints, namely boundary, monotonicity and continuity constraints [372]. Common misunderstandings about DTW include conceptions that it is too slow to be useful and that the warping window size does not matter much; Wang et al. [366] and Mueen et al. [375] have attempted to correct these notion. Kotas et 
al. [376] have reformulated the matrix of the alignment costs, which led to a major increase in the noise reduction capability. Other surveys review distance measures such as Euclidean Distance (ED) [377], Dynamic Time Warping (DTW) [378, 379], and distance based on Longest Common Subsequence (LCS) [366,380].

Correlation: is a mathematical operation widely used to describe how two or more variables fluctuate together. Different types of correlation can be found by considering the level of measurement for each variable. Distance correlation can be used as a distance measure between two variables that are not necessarily of equal dimension. In time-series data, it is used to detect a known waveform in random noise. Unlike DTW and LCS, correlation also offers a linear complexity frequency space implementation in signal processing [334,381].

Cross-correlation: is the correlation between two signals which shape a new signal, and its peaks can indicate the similarity between the original signals; it is used as a distance metric [134]. However, cross-correlation can be carried out more efficiently in the frequency domain [381]. Autocorrelation occurs when the signal is correlated with itself, which is useful for finding repeating patterns [334]. Cross-correlation might be a slow operation in time-series space [334], but it corresponds to point-wise multiplication in frequency space. It is also considered the best distance measure to detect a known waveform in random noise [334]. When processing the signal, the correlation has a linear complexity frequency space implementation which cannot be achieved by DTW.

\subsubsection{Features Extraction}

Feature extraction is a form of dimension reduction which helps to lower the computational cost of dealing with high-dimensional data and achieve higher accuracy of clustering [382]. Matching features from time-series data should be extracted before applying learning algorithms to the vector of extracted features. Several feature-based techniques have been proposed to represent features with low dimensionality for time-series data. Wang et al. [366] list several methods for reducing time-series dimensionality as feature extraction; including Discrete Fourier Transform (DFT), Discrete Wavelet Transform (DWT), Discrete Cosine Transform (DCT), Single Value Decomposition (SVD), Adaptive Piecewise Constant Approximation (APCA), Piecewise Aggregate Approximation (PAA), Chebyshev polynomials (CHEB), and Symbolic Aggregate approXimation (SAX).

Principal Component Analysis (PCA), as an eigenvalue method, is a technique which transforms the original time-series data into low-dimensional features. As a feature extraction 
method, PCA is effectively applied to time-series data [383-386]. It transforms data to a new set of variables whose elements are mutually uncorrelated, thus learning a representation of data that has lower dimensionality than the original input. PCA is a linear dimensionality reduction technique, and has been used as an effective dimensionality reduction method that eliminates the least significant information in the data and preserves the most significant. [135, $294,305,321$, 335, 338, 346, 387] use PCA to reduce high-dimensional data and analyze the similarity of time-series data.

Multidimensional Scaling (MDS): is a very popular non-linear dimensionality reduction technique that is useful for effectively representing high-dimensionality data in lower dimensional space [289, 300, 305, 307, 308, 314, 329, 332, 335]. It struggles, however, to separate Kmeans clusters [335]. Jeong et al. [289] use MDS to gain a better understanding of gene interactions and regulatory behaviors. Thus, two different MDS representations are considered with respect to time-series data. One shows local differences among genes in the same cluster group, while the other shows global differences among all genes in all the clusters. It is also used to reveal the distributions of the time-series data, helping to understand the relations among time-series [300].

K-grams: Transforming time-series data into a set of features cannot capture the sequential nature of series. K-gram is an example of a feature-based technique that aims to maintain the order of elements in series using short sequence segments of $\mathrm{k}$ consecutive symbols [368]. Kgrams [388] represent a feature vector of symbolic sequences of K-grams in time-series data. Given a set of K-grams, this feature vector can represent the frequency of the K-grams (i.e. how often a K-gram appears in a sequence).

Discrete Fourier Transform (DFT): is one of the most common transformation methods [389]. It has been used to transform original time-series data into low-dimensionality time-frequency characteristics and index them to obtain an effective similarity search [390]. DFT is used to perform dimensionality reduction and extract features into an index used for similarity searching. This technique is continually under improvement and some of its limitations have been overcome [377,391,392].

Discrete Wavelet Transform (DWT): has also been used as a technique to transform original time-series and obtain low-dimensional features that efficiently represent the original time-series data [367,393]. Chan et al. [394] use Haar Wavelet Transform for time-series indexing, which shows the technique's effectiveness with regard to the decomposition and reconstruction of time-series. With a large set of time-series data, analysis tasks face certain 
challenges in defining matching features; therefore, taking advantage of wavelet decomposition to reduce the dimensionality of data is beneficial [395]. The analysis task can be accurately performed utilizing the discrete wavelet transform technique [396].

Shapelets: Discretization is often required when applying feature-extraction techniques in time-series data, but it can cause information loss [348]. To address this, Ye et al. [397] introduce time-series shapelets which can be directly applied to time-series. This technique is based on comparing the subsection of shapes (shapelets) instead of comparing the whole time-series sequences to measure the similarity. A binary decision maker decides whether each new sequence belongs to a class or not. The shapelet classifier has some limitations with a multi-class problem, and to overcome this issue, Ye et al. [397] use the shapelet classifier as a decision tree. Xing et al. [398] show that early classification can be efficiently achieved by extracting the local shapelets features.

\subsubsection{Conventional Clustering Algorithms}

Clustering is widely used as an unsupervised learning method. The aim of time-series clustering is to define a grouped structure of similar objects in unlabeled data based on their similar features. Due to the unique structure of time-series data (e.g. high dimensionality, noise, and high feature correlation), clustering time-series differs from traditional clustering, consequently, several algorithms have been improved to deal with time-series. Most works involving the clustering of time-series can be classified into three categories [134]. The first is whole time-series clustering, where a set of individual time-series is given and the aim is to group similar time-series into clusters with respect to their similarity. The second is subsequence clustering, which involves dividing the time-series data at certain intervals using a sliding window technique to perform clustering on the extracted subsequences of a time-series. The third category is a clustering of time points based on a consolidation of their temporal proximity and the similarity of the corresponding values. Some points might not assign to any clusters and are deemed as noise. Chapter 2 provided a review of popular clustering algorithms (see section 2.3.1 for detailed descriptions of these methods). The discussion about various types of methods discussed below aims to review clustering algorithms used for time-series data.

\subsubsection{Partitioning Methods}

Partitioning methods are described as the process of partitioning unlabeled data into $K$ groups. Kmeans [121] , Kmedoids (PAM)) [120], Fuzzy Cmeans [126,127], and Fuzzy Cmedoids [132] 
are the most popular algorithms for partitioning clustering. Kmeans has been used to cluster time-series data, achieving efficient clustering results due to its speed, simplicity, ease of implementation, and the possibility to assign the desired amount of clusters [123, 124]. The Kmedoids or PAM (partition around medoids) algorithm is often used alongside the DTW distance measure to cluster time-series data [399]. Andrienko et al. [320] used Kmedoids as a clustering algorithm, which could be better suited than Kmeans, as it uses medoids instead of means. However, it still has the same issues as Kmeans, where the number of clusters must be known in advance. Unsupervised partitioning has been shown to be as efficient at providing good clustering accuracy for time-series clustering. Several partitioning clustering approaches (e.g. Kmeans [123, 355, 399, 400, 400, 401], Kmedoids [402], Fuzzy Cmeans [129,403], and Fuzzy Cmedoids [404]) have been used to achieve efficient clustering results for sequences of time-series data.

\subsubsection{Hierarchical Methods}

Hierarchical clustering defines a tree structure for unlabeled data by aggregating data samples into a tree of clusters. This method does not assume a value of $\mathrm{K}$, unlike Kmeans clustering. There are two main kinds of hierarchical clustering methods - agglomerative (bottom-up) and divisive (top-down) $[133,134]$. The hierarchical method is applied to determine the order of time-series data [292,387]. Wijk et al. [405] conducted pioneering work in which they use a bottom-up hierarchical clustering approach to identify common and uncommon subsequences that occur in large time-series. Battke et al. [288] overcame the issue of hierarchical clustering speed for large time-series datasets by implementing the rapid neighbour-joining algorithm [406].

\subsubsection{Model Based Methods}

A self-organizing map (SOM), a model-based method developed by Kohonen [144], is a specific type of neural network $(\mathrm{NN})$ used for model-based clustering. SOM has been used to analyze temporal data and is utilized for pattern discovery in temporal data [135, 288, 296, 297, 321,330,407]. The introduction of Recurrent SOM [408] and Recursive SOM [409] has enhanced SOM for mapping time-series data [156]. Fuet et al. [410] use self-organizing maps to gather similar temporal patterns into clusters. A continuous sliding window is used to segment data sequences from numerical time-series before applying the SOM algorithm. SOM is also used in [157] to cluster time-series features. Many works on clustering have chosen 
SOM due to its advantages with regard to certain properties such as parameter selection and data analysis. However, one of its main disadvantages is that it does not work perfectly with time-series of unequal length, as it is difficult to define the dimension of weight vectors [155].

\subsubsection{Density-Based Methods}

In density-based clustering, the cluster continues to expand if the density of a set of points with its neighbors is closely packed together, and that cluster is separated by subspaces where the objects have low density. Density-based clustering for time-series data has some advantages; it is a fast algorithm which does not require pre-setting the number of clusters, is able to detect arbitrarily shaped clusters as well as outliers, and uses easily comprehensible parameters such as spatial closeness [329]. Although density-based clustering entails some complexity, many time-series clustering algorithms have adopted this method [288, 295, 300, 308, 315, 317, 318, $320,322,324,325,328,329,340]$.

\subsection{DeepCluster Method Applied to Biological Time-series Data: A Case Study}

The process of time-series clustering is accompanied by several difficulties and challenges, such as feature representations at different time scales, and distortion by high-frequency perturbations and random noise in time-series data [411]. Time-series data has also shown considerable diversity in relevant features and properties, dimensionality, and temporal scales [412]. To overcome these challenges, a deep learning method can be designed to disentangle the data manifolds and allow a clustering method to deal with learned features instead of raw data. Traditional clustering algorithms tend to attain limited performance as dimensionality increases. Dealing with high-level representation provides benefits that support the achievement of clustering tasks. Deep clustering allows a deep neural network to extract similar patterns in lower-dimensional space and find idealistic representative centers for distributed data. Efforts have been made in the field of computer vision in developing deep clustering methods for image datasets. Deep auto-encoders (DAEs) and deep convolutional auto-encoders (DCAEs) are unsupervised models. These models have been exploited for clustering, where features learned through deep networks provide an abstracted latent representation used for clustering analysis. The previous works can be categorized into four different categories, summarized in Table 7.1. 


\begin{tabular}{|r|l|l|}
\hline Method & Separated Clustering & Embedded Clustering \\
\hline DAE & Tian et al. [168], Huang et al. [169] & Song et al. [167], Xie et al. [171] \\
\hline DCAE & Li et al. [174], Guo et al. [175] & Alqahtani et al. [5] \\
\hline
\end{tabular}

Table 7.1: Deep Clustering Methods. Separated Clustering consists of two main steps, where they extract latent features for given data and then perform clustering on the learned representations. while embedded Clustering methods have embedded a clustering algorithm into deep neural networks, where feature representations and clustering assignments are simultaneously learned, applying joint loss function.

DeepCluster [5] is an unsupervised clustering method that simultaneously captures representative features and the relationships among images. The goal is to learn feature representations and cluster assignments simultaneously, employing the strength of DCAE to learn high-level features. Two objective functions were integrated together: one minimizes the distance between features and their corresponding cluster centers, while the other minimizes the reconstruction error of the DCAE, defined as follows:

$$
\min _{W, b} \frac{1}{N} \sum_{n=1}^{N}\left\|x_{n}-\hat{x}_{n}\right\|^{2}+\lambda \cdot \frac{1}{N} \sum_{n=1}^{N}\left\|h^{t}\left(x_{n}\right)-c_{n}^{*}\right\|^{2},
$$

where $N$ denotes the number of samples, $\hat{x}$ is a reconstructed sample, and $x$ is an original sample, $\lambda$ controls the contribution of the clustering cost function, $h^{t}(*)$ is the internal representation obtained by the encoder mapping at the $t^{\text {th }}$ iteration, $x_{n}$ is the $n^{\text {th }}$ sample in the dataset, and $c_{n}^{*}$ is the assigned cluster center to the $n^{\text {th }}$ sample. During optimization, all data representations are assigned to their new identical cluster centers, after which the cluster centers are updated iteratively, allowing the model to achieve stable clustering performance. The defined clustering objective, as well as the reconstruction objective, are simultaneously used to update the parameters of the transforming network. DCAE might be well-suited to time-series data because it captures the time-series' shape and allows local shift-invariance. This section applies what was proposed in Chapter 3 to real-world time-series data; namely, the Imperial Cormorant bird dataset (ICBD) from the Biosciences department at Swansea University. The experimental architectures of DAE and DCAE (Section 7.4.1) will be discussed and the Imperial Cormorant Birds Dataset (ICBD) described before the preparation of time-series data is highlighted (Section 7.4.2), and our experimental results outlined (Section 7.4.3).

\subsubsection{Network Architectures for ICBD}

Our method is designed to cluster large time-series data using deep neural networks. In this section, we introduce our experimental architectures of two types of neural networks: DAE 


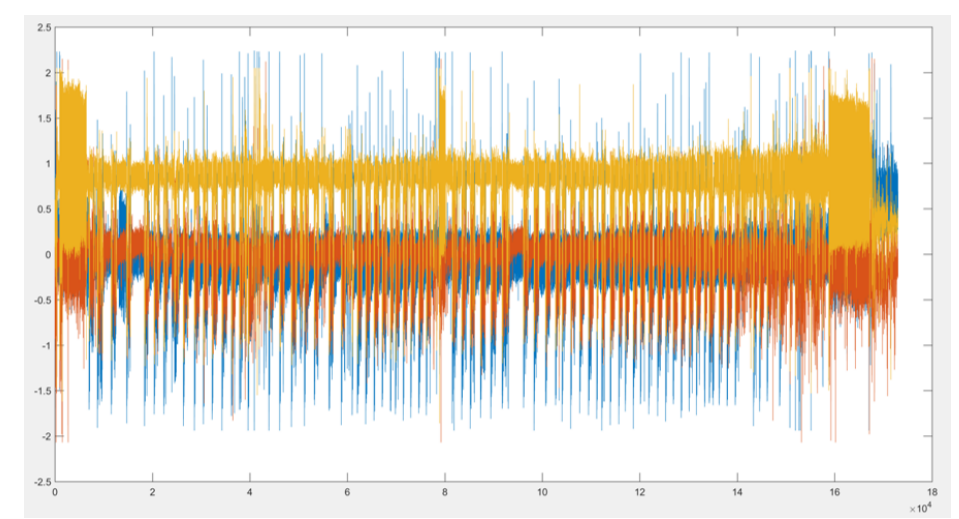

Figure 7.2: Line chart of the raw accelerometer data (Multivariate time-series data).

and 1D-DCAE. Through such deep learning models, we study the impact of learned features, via fully-connected neural networks or convolutional neural networks, to improve clustering quality.

\subsubsection{Deep Auto-encoder (DAE)}

DAE is an unsupervised model for representation learning. It maps inputs into new space representations, providing useful features through its encoding procedure. As the raw data is transformed into a more abstract representation, our embedded clustering algorithm can deal with the learned features. We built a deep architecture of a series of signal-processing fullyconnected layers for feature extraction, consisting of multiple fully-connected layers, each composed of a set of linear/nonlinear units.

The DAE architecture consists of seven fully-connected layers with 30 neurons in the first layer, 20 neurons in the second layer, and 10 neurons in the third layer. This is followed by 5 neurons as a result of the encoding part. The decoding part utilizes three fully-connected layers. The first consists of 10 neurons, the second of 20 neurons, and the third of 30 neurons. We exploit the learned features via the internal layer and feed it to clustering loss function, which minimizes the distance between data points and their assigned cluster centers, embedding Kmeans clustering algorithm into the DAE framework. The detailed configuration of the DAE network architecture used in the experiments is shown in Fig. 7.3. ReLU is utilized as a standard activation function. 


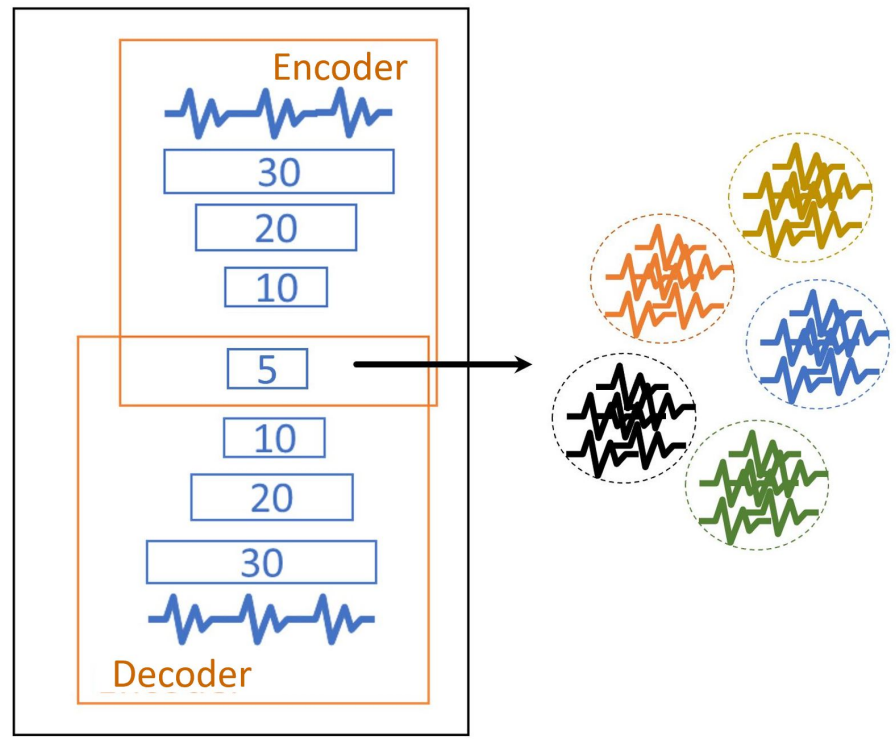

Figure 7.3: The DAE network architecture for time-series data shown the number of neurons for each fully-connected layer in both encoder and decoder parts.

\subsubsection{1D-Convolutional Layer for Deep Convolutional Auto-encoder (1D-DCAE)}

Unlike 2D grid (e.g. image data) input, convolutional layers for time-series data use a 1D grid, so instead of holding raw 2D pixel values, the input of time-series data is multiple 1D subsequences. In this case, multivariate time-series [69] are separated into univariate ones so that feature learning can be performed for each univariate series. In other words, the multivariate time-series are considered as input that is fed into the convolutional layers, learning features through convolution and activation layers. The 1D-convolutional layer extracts features by applying dot products between transformed waves and a 1D learnable kernel (filter) [411], computing the output of neurons that are connected to local temporal regions in the input. This stage is followed by the activation layer, which is used to perform non-linearity within the networks, allowing for the learning of more complex models [413]. After extracting feature maps from multiple channels, they are fed into other convolutional layers and then passed as inputs of the fully-connected layer. In the fully-connected layer, the learned feature representations are fed to the clustering loss function via the internal layer of DCAE, which embeds a clustering algorithm into the body of a DCAE model.

The architecture of DCAE consists of three 1D-convolutional layers with filter sizes of $10 \times 1$ with 32 kernels in the first convolutional layer, 64 kernels in the second convolutional 
Table 7.2: Detailed configuration of the DCAE network architecture used on time-series data.

\begin{tabular}{|c|c|}
\hline Layer & Biological Dataset \\
\hline Convolutional & $10 \times 1 \times 32$ \\
\hline Convolutional & $10 \times 1 \times 64$ \\
\hline Convolutional & $10 \times 1 \times 128$ \\
\hline Convolutional & $1 \times 3 \times 128$ \\
\hline Fully-connected & 384 \\
\hline Fully-connected & 5 \\
\hline Fully-connected & 384 \\
\hline Deconvolutional & $1 \times 3 \times 128$ \\
\hline Deconvolutional & $10 \times 1 \times 128$ \\
\hline Deconvolutional & $10 \times 1 \times 64$ \\
\hline Deconvolutional & $10 \times 1 \times 32$ \\
\hline
\end{tabular}

layer, and 128 kernels in the third convolutional layer. This is followed by two fully-connected layers, which have 384 and 5 neurons respectively, in the encoding part. In the decoding part, a single fully-connected layer of 384 neurons is followed by three 1D-deconvolutional layers. The first deconvolutional layer consists of 128 kernels, the second deconvolutional layer consists of 64 kernels, and the third deconvolutional layer consists of 32 kernels. The detailed configuration of the DCAE network architecture used in the experiment is presented in Table 7.2. ReLU is utilized as a standard activation function.

\subsubsection{Imperial Cormorant Birds Dataset (ICBD) and Pre-processing}

Animal behavior analysis has received considerable attention in this area of interest, where 'smart' sensors (i.e. accelerometers) attached to wild animals have revolutionized biologists' understanding of their ecology. A tri-axial accelerometer is one preferred source of quantitative data to identify animal behavior through movement. Biologists widely use accelerometers as they help them monitor and determine wild animals' behaviors [414] in their natural environment over long periods of time. The attachment of a tri-axial accelerometer provides analyzed data, which allows researchers to investigate an animal's movement through identifying its posture and changes in its body velocity [334], revealing much about its behavior [415]. Directly dealing with multiple sensors at high frequencies is expensive and requires 
expert knowledge $[414,416]$. Previous efforts by biologists have been made to analyze raw accelerometer data, where Overall Dynamic Body Acceleration (ODBA) [417] and Vectorial Dynamic Body Acceleration (VeDBA) [418] have been proposed as surrogate measures for speed. The VeDBA appears more robust than the ODBA because it provides values closer to the true physical acceleration experienced and copes better than ODBA with variability in substrate [418]. Therefore, we calculate the VeDBA to derive new acceleration values from tri-axial accelerometer data using the following form:

$$
V e D B A=\sqrt{D A_{x}^{2}+D A_{y}^{2}+D A_{z}^{2}}
$$

where $D A_{x}^{2}, D A_{y}^{2}$, and $D A_{z}^{2}$ denote the dynamic acceleration values obtained by taking the absolute values of running means of the raw acceleration values of each of the accelerometer's 3 axes from the corresponding raw acceleration values.

The Imperial Cormorant bird dataset (ICBD) was provided by biologists from the Biosciences department at Swansea University. This dataset contains more than $173 \mathrm{~K}$ data points associated with a label from 5 different classes (descent diving, bottom diving, ascent diving, swimming, and flying). Fig. 7.2 presents the raw accelerometer data.

\subsubsection{Normalization}

In our case, each dimension of the time-series data is normalized using unity-based normalization where all values are set in a range between $[0,1]$ using the following form:

$$
x^{\prime}=\frac{x-\min (x)}{\max (x)-\min (x)}
$$

\subsubsection{Sliding Window Approach}

A sliding window approach was used to segment continuous time-series data into a set of short segments. The sliding window technique convolves along the time axis based on two parameters (i.e. window size $W$ and stride $S$, which is the step size of sliding a window). Here, the window size $W$ is a determined sampling rate. A fixed sliding step of 30 is adopted in our experiments, and stride $S$ is set to 15 as recommended by the biologists. However, a smaller value for stride can be chosen to increase the number of samples in the dataset and would be useful to capture more local temporal patterns and avoid lost data, smoothing the transition between time-steps. Fig. 7.4A. and Fig. 7.4 present the sliding window approaches which were used in our experiment. The sliding window approach is applied to two different 
categories of time-series data: univariate time-series data (Fig. 7.4A.) and multivariate timeseries data (Fig. 7.4).

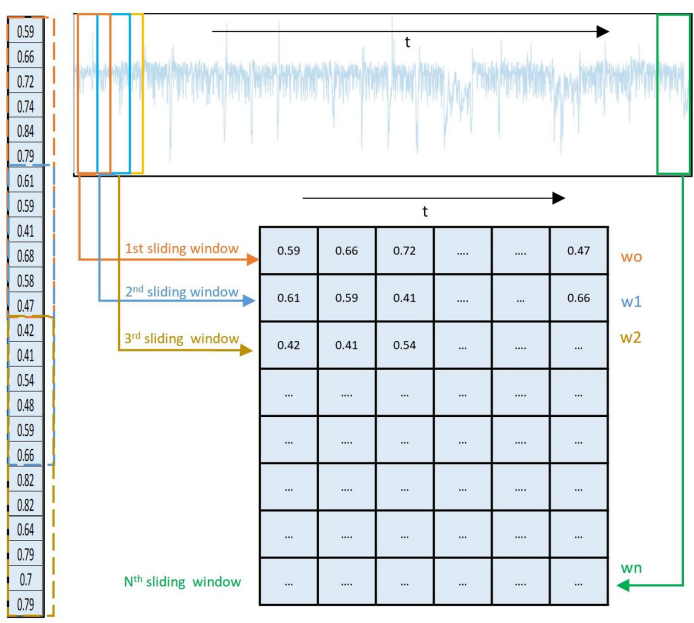

A. Univariate Time-series Data

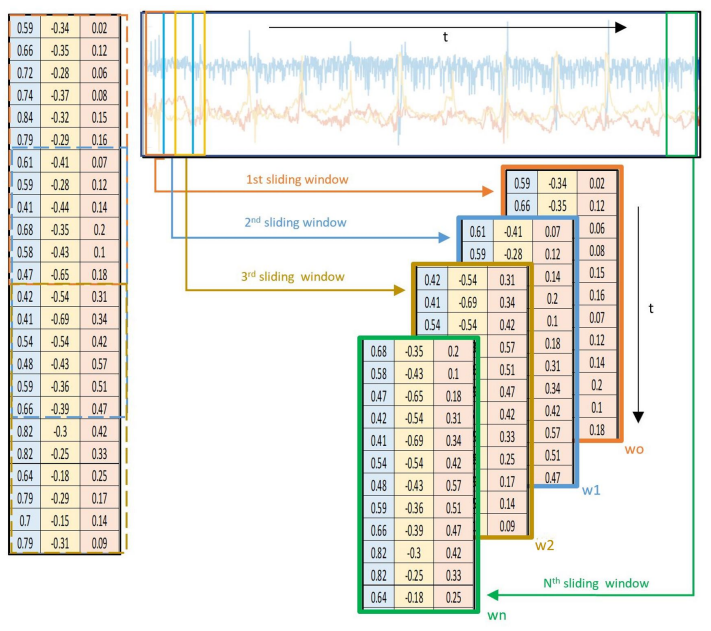

B. Multivariate Time-series Data

Figure 7.4: The detailed process of the sliding window approach with a window size of 12 and and a stride of 6 are adopted in both cases.

\subsubsection{Experiment and Discussion}

\subsubsection{Experiments Setup}

The proposed method was implemented using MatConvNet [419] in Matlab. As a result of the complexity and variability characteristics of the ICBD dataset, obtaining reliable training data normally requires collecting multiple annotations from different experts and then performing cross-validation on the collected labelings. We performed 5-fold cross-validation on the provided classes, splitting them into 5 equal subsets. In each evaluation round, each model was trained on 4 folds and tested on the 5th one. This procedure was repeated for all 5 folds. Both sliding window approaches were applied to extract subsequences from the folds. Moreover, the VeDBA method was applied to the raw tri-axial accelerometer data using Eq.(7.2) to obtain univariate time-series data. Following this, the univariate time-series data was segmented to be used as input data for the Kmeans and the DAE framework, while the multivariate time-series data was segmented to be used as input data for the DCAE.

The model was trained end-to-end in an unsupervised manner, with no pre-training or fine-tuning procedures involved. All weights were initialized using the Xavier method [57], 
Table 7.3: Experimental results of clustering quality, reporting averaged performance across 5-fold cross-validation on three methods on the ICBD.

\begin{tabular}{r|c|c|}
\hline & ACC & MNI \\
\hline Kmeans & 37.44 & 19.73 \\
\hline DAE with embedded clustering & 78.67 & 53.63 \\
\hline DCAE with embedded clustering & $\mathbf{9 4 . 3 6}$ & $\mathbf{7 9 . 4 0}$ \\
\hline
\end{tabular}

biases were set to 0 , and the cluster centers were initialized randomly. Stochastic gradient descent with mini-batch was used, where each batch contained 32 random shuffled instances. Furthermore, an initial learning rate of 0.006 with a momentum of 0.9 and weight decay of 0.0005 was used. We set $\lambda$, the clustering weight-parameter that controls the loss contribution percentage of clustering error, to 0.1 , and the model converged after 100 epochs.

\subsubsection{Experimental Results}

To evaluate cluster quality, two evaluation metrics, accuracy (ACC) and normalized mutual information (NMI), were computed. We compared three different methods: Kmeans, DAE with embedded clustering, and DCAE with embedded clustering. The results are promising, showing the latent space encodes sufficient patterns to facilitate accurate clustering of animal behaviors through movement. Table 7.3 demonstrates that DCAE with embedded clustering outperforms the other methods, where $79.40 \%$ and $94.36 \%$ were achieved on NMI and ACC respectively. It also shows the performance of the clustering algorithm in different spaces, i.e. the original data space and the hidden space learned via non-linear mapping with both DAE and DCAE. The experimental results of the traditional Kmeans support our hypothesis that conventional clustering algorithms attain limited performance as dimensionality increases. Within the latent space of AE, the clustering algorithm benefits from the DAE, which allows it to deal with learned features rather than raw data. With regard to local temporal information via DCAE, the local salience of the signal shows its ability and allows the clustering algorithm to perform much better. DCAE allows local capture of the salience of signals and the obtaining of the specific variance of signals at different scales, which helps the clustering algorithm deal with the more clustering-friendly representation. It also shows that univariate representation of data in K-means and DAE lost information compared with the multivariate analysis in DCAE. 


\subsection{State-of-the-art and Outlook}

Since we applied our DeepCluster method to time-series data, deep learning-based clustering methods have become a novel trend and are increasingly adopted in time-series applications with various designs of deep network architectures and clustering methods from several application domains. We review these works, identifying state-of-the-art, and present an outlook on this important field of deep time-series clustering (DTSC) from five important perspective. We believe that the following aspects of DTSC are worthy of further investigation, and could open up promising research directions.

\subsubsection{Different Network Architectures}

Since 2018, the DTSC has received particular attention with regards to different kinds of network achitecture, such as deep auto-encoder (DAE) [420-423], deep convolutional autoencoder (DCAE) [424-431], and recurrent neural networks (RNNs), including RNN autoencoder (RNN-AE) [432-435] or seq2seq auto-encoder (S2S-AE) [436-438]. DTSC can be considered to fall into two pipelines (see Fig. 7.5): the sequential multi-step approach or joint approach. The sequential multi-step approach consists of two main steps (see Fig. 7.5A.); the first step learns efficient representations of the time-series data through deep networks, while the second step performs clustering on the learned representations. In the joint approach, learning time-series representation and the clustering process are integrated into a single network model, allowing the extraction of latent features and cluster assignments simultaneously (see Fig. 7.5B.).

Two significant steps separate the clustering task from representation learning and feature extraction. Thinsungnoen et al. [420] apply a DAE to learn efficient time-series representatives, demonstrating that time-series data of ECG signals reveals useful hidden information. The learned ECG representations are then fed to an agglomerative hierarchical approach for the clustering process. In the same manner, a DCAE was used to extract latent features of timeseries under the influence of temporal distortion [429], demonstrating that the learned latent space is suitable for Kmeans clustering. The DCAE was also utilized by [430] to map the data of the yearly load profile into a low-dimensionality representative vector. A Kmeans clustering algorithm was then carried out based on the learned vectors. In [425], ECG records also benefit from deep clustering, where a GMM clustering metric is optimized in the lower-dimensional latent space of DCAE. These techniques are applied to time segments of continuous wavelet 


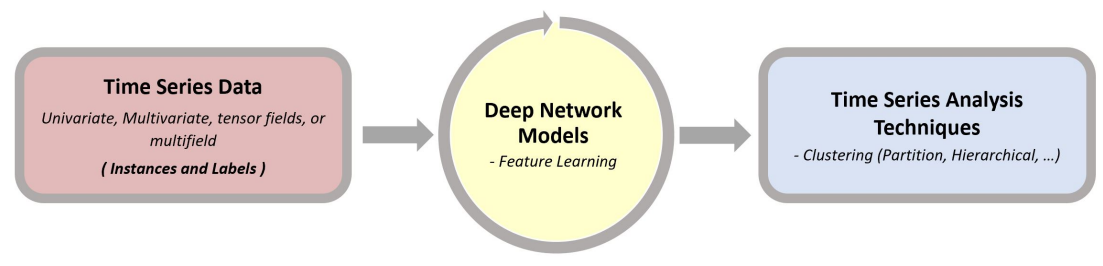

A. Multi-step deep time-series clustering

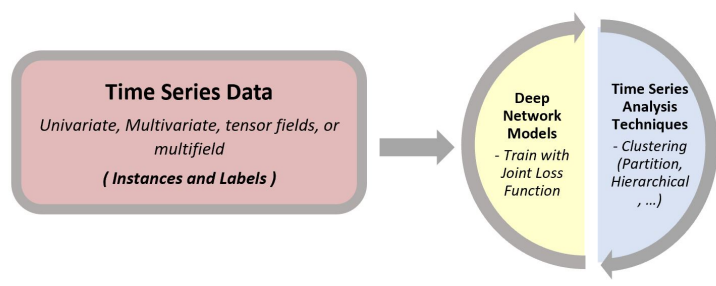

B. Joint deep time-series clustering

Figure 7.5: The pipelines of deep time-series clustering.

transforms of ECG signal, representing a diversity of health conditions. A 1D-convolutional layer's architecture was also adopted for a deep clustering method [427] to cluster the operating conditions of a system and identify the fault signals not associated with the new conditions clusters. In addition, Guillaume et al. [428] propose 1D-DCAE to learn the features of timeseries data, which are used as input to a Kmedoids algorithm to perform clustering.

Deep neural networks with embedded clustering have been developed, which simultaneously allow extracting features and clustering assignments within the training process. Inspired by deep image clustering [171], Sai et al. [424] propose deep temporal clustering (DTC), which uses DAE as an initialization method to learn feature representations and indirectly perform clustering. The clustering layer is designed to optimize a Kullback Leibler (KL) divergence objective to enforce a self-training target distribution. The encoding procedure can control the clustering performance, since the predicted distribution is estimated based on the learned representations which are later fed to Kmeans for clustering. The concept of deep clustering for static image datasets was also transferred to multivariate time-series data by [426], where 1DDCAE was utilized to help latent space clustering. High-dimensional time-series data poses some difficulties when looking to effectively model traffic patterns; thus, deep clustering has been employed to jointly perform representation learning and clustering of a large unlabeled dataset [439]. Sun et al. [421] adopted a deep embedded clustering to jointly extract new features and form the clusters for household load in demand response application. Moreover, Lee 
and Schaar [434] have introduced a deep learning approach for clustering time-series data using a method which consists of several networks: an encoder, a selector, a predictor, and an embedding dictionary. Together, these components provide the cluster assignment and the corresponding centroid based on a given sequence of observations through optimizing joint loss functions. This encourages each cluster to have homogeneous future outcomes (e.g., adverse events, the onset of comorbidities, etc.).

Recurrent Neural Network (RNN) [12,93] and Long Short-Term Memory (LSTM) [440] are the most commonly used techniques for time-series analysis tasks, particularly in supervised learning. However, RNN hasrecently been exploited for unsupervised clustering. Ienco et al. [432] propose a multivariate time-series clustering method utilizing RNN in a method which employs a Gated Recurrent Unit [441] to encode time-series data into a new vector embedding representation, based on which a centroid-based clustering algorithm (i.e. Kmeans) is applied on the new data representation. Like the mechanism of a traditional auto-encoder, the RNN encoder maps inputs into a new representation space. The data is projected into a set of feature spaces, using the encoding part, from which a recurrent decoder reconstructs the original data. Yue et al. [433] adopted an RNN auto-encoder to jointly learn embedding latent space behaviors. A clustering-oriented loss is directly built on the embedded features to cluster assignments. The same architecture was adopted by Abedin et al. [436] for human activity recognition. The encoder maps a windowed excerpt of a raw multi-channel sensory sequence into a fixed-length representation as a holistic summary of the input. Once the DAE is pre-trained, a parameterized clustering network is applied as an extension to the framework to refine the latent space and guide the network towards yielding clustering-friendly representations.

The seq2seq [442] is an unsupervised encoder-decoder based model able to learn representations from sequence data, exploiting labels to support the learning process [443]. Two RNNs work together with a unique token, attempting to predict the next state sequence from the previous one. The seq2seq is used by Kiros et al. [444] to learn the sentence representations and predict the context sentences of a given sentence. Gan et al. [445] also used the seq2seq model to predict multiple future sentences. Their experiments demonstrated the benefits of a task-related representation, where model performance can be significantly improved by finetuning with a downstream classification task. Motivated by this, Ma et al. [437] proposed deep temporal clustering representation (DTCR), where the learned representations facilitate the clustering task. The original time-series data is mapped through an encoder procedure into la- 
tent space representations, which are used to reconstruct the original shape with a decoder part. At the same time, a Kmeans objective is embedded into the model, allowing latent features and clustering assignments to be learned simultaneously.

Section 7.4 focused on DAE and DCAE and proposed a deep time-series clustering (DTSC). We were inspired by the results of our proposed method of a deep convolutional auto-encoder with embedded clustering applied to image datasets. Following a similar line of thinking, researchers can further adapt the neural architecture advances in deep clustering in the field of computer vision to satisfy time-series data. However, we argue that there is no ultimate architecture to DTSC, thus, it is a strong starting point to study how various architectures could solve a particular DTSC problem.

Most of the previously described methods rely on the capabilities of the encoder, so the focus is on the auto-encoder architectures. Deep clustering with generative adversarial networks (GANs) [89] for time-series data is a research direction of interest. To the best of our knowledge, time-series clustering tasks have not exploited the full power of GANs, even though they have received attention in the field of computer vision and image processing. For example, GAN has been adopted for clustering by Mukherjee et al. [446], who proposed ClusterGAN, a GAN-based image clustering method. They recovered latent features of image data, exploiting the unconditional GAN to effectively achieve unsupervised clustering in the latent space. The latent variables from a mixture of encoded variables (i.e. one-hot encoded vectors) are jointly trained with clustering-specific loss. Although ClusterGAN has achieved state-of-the-art in the computer vision community, it is currently under-represented in DTSC works. Furthermore, the original GAN was extended to model realistic time-series data [447], demonstrating that time-series GAN (TSGAN) can be a better generator and produce high fidelity and diverse synthetic time-series with low to limited training data. Benefiting from TSGAN, the utilization of ClusterGAN could be applied to the DTSC; this could result in promising future work.

\subsubsection{Different Clustering Methods}

Examining the clustering methods utilized in DTSC, the papers surveyed indicate that the trend is dominated by Kmeans as a commonly applied partitioning method of clustering [421, 423, $424,426,427,429-439]$. The reason for this may be due to its speed, simplicity and ease of implementation. In [426], soft-dynamic time warping (SDTW) [448] was used as an alternative similarity measure to Kmeans, allowing for the management of dissimilarity evaluation of two time-series of variable length. The SDTW is a smooth formulation of DTW recently introduced 
Table 7.4: Deep Time-series Clustering Methods. RL Indicates Reconstruction loss

\begin{tabular}{|c|r|c|c|c|c|c|}
\hline Categories & DTSC Methods & Year & Architecture & Loss & Clustering Methods & Data Type \& Applictions \\
\hline \hline \multirow{5}{*}{ Multi-step } & DAN-ECG [420] & 2018 & DAE & RL & Hierarchical & ECG \\
& RLPC-DCAE [430] & 2018 & DCAE & RL & Kmeans & Load Forecasting \\
& DEC-ECG [425] & 2019 & DCAE & RL & GMM & ECG \\
& CSS-DCAE [429] & 2019 & DCAE & RL & Kmeans & Seismology \\
& STTP-DC [439] & 2019 & CNN & Multi & Kmeans & Traffic Analysis \\
& AE-TSC [428] & 2020 & DCAE & RL & Kmedoids & Energy (Demand Response) \\
& TCN-SDF [427] & 2020 & 1D-DCAE & RL & Kmeans & Operating Machinery \\
& DM-TSEC [432] & 2020 & RNN-AE & RL & Kmeans & ECG \\
& IBS-DC [435] & 2020 & RNN-AE & RL & Kmeans & Animal Motion \\
& DPC-DP [434] & 2020 & RNN-AE & Multi & Kmeans & Disease Progression \\
\hline \hline \multirow{6}{*}{ Joint } & DTC [424] & 2018 & 1D-DCAE & RL & Kmeans & UCR Archive datasets \\
& DL-CMCPT [422] & 2019 & DAE & RL & GMM & Disease Progression \\
& C-RBE [421] & 2019 & DAE & RL & Kmeans & Energy (Demand Response) \\
& STC-TD [423] & 2019 & DAE & RL & Kmeans & Traffic Analysis \\
& IDTC [426] & 2019 & DCAE & RL & Kmeans & Automotive Diagnostic \\
& DETECT [433] & 2019 & RNN-AE & RL & Kmeans & Mobility Analysis \\
& LR-TSC [437] & 2019 & S2S-AE & RL & Kmeans & ECG \\
& TC-TCM [438] & 2020 & RNN-AE,DCAE & RL & Kmeans & Thermal Condition Monitoring \\
& DC-HA [436] & 2020 & RNN-AE & RL & Kmeans & Human Activities \\
\hline
\end{tabular}

to overcome the computational costs of DTW [449]. Other partitioning clustering methods can be efficiently applied to DTSC, such as Richard et al. [428] using a Kmedoids algorithm to cluster time-series data on the latent space due to its simplicity and robustness to outliers.

Although popular conventional clustering methods (i.e. hierarchical, model-based, and density-based clustering) have achieved efficient clustering results, they have rarely been used as clustering methods in DTSC frameworks. Driven by the achievements of these conventional methods, exploring the usefulness of adopting them in DTSC is a suggested path. This would open another interesting direction for researchers, as the powerful non-linear transformation would benefit these methods' performance. For instance, self-organizing maps (SOM) [144] have rarely been used as a clustering algorithm for DTSC. Embedding SOM into the latent space would allow modeling of the latent space and joint learning of the latent representations and code vectors of SOM.

\subsubsection{Deep Learning Heuristics}

As one of the concrete examples, data augmentation can help a model learn features that are invariant to transformation and can support learning using the transform invariant approach to facilitate the job of DTSC in producing a significant performance. We believe there is considerable research potential in developing specific augmentation techniques for time-series data, where the temporal aspect of the data can be considered. For instance, Weber et al.'s learnable 
warping functions [450] can be leveraged so the network can learn the optimal warping features by adopting continuous affine and more complex transformations, which can improve the performance of DTSC.

The use of time-series clustering is due to the lack of labels in such data. Supervision knowledge dramatically assists the formation of discriminative transformations learned by the encoding part of the DCAE, ameliorating the clustering algorithms in the latent space [4]. Even weak or partial supervision knowledge could significantly improve the quality of DTSC. Since semi-supervised learning has allowed us to leverage a large number of unlabeled images efficiently, we assume that DTSC researchers would benefit from adopting this type of procedure to more efficiently guide a large amount of unlabeled time-series data toward obtaining more discriminative data partitioning.

\subsubsection{DTSC Applictions}

Concerning our classification of time-series data in section 7.2, DTSC is applied to different time-series data types from various applications. Multivariate time-series data including disease progression [422, 434], ECG signals [420,425, 432], demand response [421, 428], load forecasting [430], pattern changes in temperature [438], and seismic signal [429], made use of DCAE. Moreover, DTSC provided a great benefit to tensor fields' data type, including machines (e.g. engines), which typically consist of a large number of sensors or nodes that produce vast amounts of data collected over a period of time [426,427]. In time-series of spatial positions of moving objects, trajectory data presents different places over time, providing a clear idea of spatio-temporal changes. The DTSC method is applied to cluster this type of application to understand and recognize the mobility of a range of moving objects, such as vehicles [423,439] and spacecrafts [424], which can lead to path discovery, movement analysis, and location prediction. Discovering behavioral patterns and finding interesting events in certain municipalities' sectors is considered spatial configurations and distributions. This type of application (i.e. personal mobility behaviors [436] and movement patterns [431,433], and behaviors of animals [435]) has also benefited from DTSC. Time-series data pose challenges for real-world applications because of the data acquisition method and the inherent nature of such data [412]. Based on the aforementioned architectures, methods, and applications, we believe that it would be possible to enable more application domains to access the significant gains of DTSC. For instance, a wide range of applications in human motion capture and action recognition [451] can benefit from DTSC. Thus, it would be of considerable interest to explore 
how such applications can make use of DTSC and how its abilities can be improved.

\subsubsection{DTSC Benchmarks}

DTSC has been applied to various applications, and we believe it will have an influence on even more application domains in the future, in the same manner as conventional clustering algorithms. The UCR time-series archive [452] has become the state-of-the-art repository of time-series data and an essential resource for the time-series data mining community. The limitation associated with testing time-series clustering algorithms is studied by [453], utilizing all time-series datasets available in the UCR archive for popular conventional clustering methods (i.e., partitional, hierarchical, and density-based, discussed in section 7.3.2). Beyond presenting new review papers, especially for DTSC, we believe the generalization of this time-series clustering benchmark to include DTSC methods warrants further study. This can present a useful reference for the research community, and dataset-level assessment metrics can be used to validate the newly proposed methods.

\subsection{Summary}

As has been shown, deep clustering of time-series data comes with several challenges under continual study. This chapter has explicitly examined automatic methods, with a focus on time-series data and machine learning clustering techniques as part of deep time-series clustering (DTSC). A comprehensive review of time-series data analysis was provided, focusing on time-series data and several choices of similarity measures and feature extraction, which significantly influence the quality of analysis techniques. Time-series clustering faces obstacles and difficulties, such as feature representations at different time scales, and the potential for distortion by high-frequency perturbations, random noise in time-series data, and increasing dimensionality. These challenges can make the detection of interesting patterns very difficult for traditional clustering algorithms, but this can be overcome by the adoption of deep learning. We explored the topic of DTSC for the first time and presented a case study. We applied what we proposed in Chapter 3 to real-world time-series data in the form of the Imperial Cormorant bird dataset (ICBD) from the Biosciences department at Swansea University. We subsequently reviewed other recent state-of-the-art methods, discussed the challenges of DTSC, suggested opportunities and potential future directions for research, and presented an outlook of the field of DTSC from five important perspectives. Finally, as deep learning has attained extraordinary 


\section{Deep Time-Series Clustering}

achievement in numerous machine learning fields, especially in computer vision, text mining, speech recognition, and image segmentation, we believe that there is ample scope for DTSC researchers' exploration, as deep learning models have advanced extremely quickly. We hope this chapter can act as a keystone for future research on DTSC.

In the following chapter, we will summarize the presented works, drawing conclusions on the use of deep representation and its strength in deep clustering and deep network compression. We will consider the future of both fields and highlight potential research directions. 


\section{Chapter 8}

\section{Conclusions and Future Work}

\section{Contents}

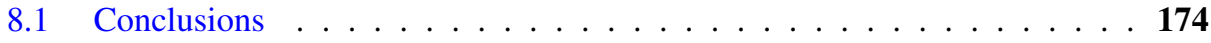

8.2 Future Work $\ldots \ldots \ldots \ldots \ldots \ldots \ldots \ldots \ldots$ 


\subsection{Conclusions}

This study explored the use of representation learning in deep clustering and deep network compression. Learned representation has a demonstrated ability to enhance unsupervised clustering and help determine substantial parts of a network when compressing and accelerating deep networks. We initially presented DeepCluster, which embeds clustering approaches in a deep convolutional auto-encoder (DCAE) for efficient, simultaneous, end-to-end learned local features and cluster assignments. From this study, we identified the necessity of exploring the use of deep clustering in the presence of varying degrees of discriminative power. We also presented a novel framework to measure the importance of individual hidden unit representations, quantifying interpretability for more robust and effective CNN pruning, and proposed an iterative pruning method that prunes neurons based on their level of importance during training. Lastly, we explored the use of a DeepCluster to learn and cluster temporal features from accelerometer data for the clustering of animal behaviors, and discussed the current state of deep time-series clustering approaches, the challenges and opportunities associated with them, and future directions.

We introduced an unsupervised deep clustering method where a non-linear latent representation and compact clusters are learned jointly in Chapter 3. We proposed two clustering methods embedded in a deep convolutional auto-encoder (DCAE). DCAEs have been effective in image processing, as they fully utilize the properties of convolutional neural networks. Our methods consist of clustering and reconstruction objective functions. All data points are assigned to their new corresponding cluster centers during the optimization, after which clustering parameters are iteratively updated to obtain a stable performance of clustering. We demonstrated the effectiveness of our clustering methods by comparing them with seven baseline methods on three image datasets. The experimental results showed that DCAE-GMM substantially outperforms all other deep clustering models, and far better than DCAE-Kmeans, as the DCAE-Kmeans model failed to identify some outliers samples, while DCAE-GMM classified them effectively, looking qualitatively identical to the ground truth with visual analysis. The visual assessments showed that as the learning scheme continues, the overlapping clusters become discriminative and enforce compact representation, and the inter-cluster distances are enlarged, which indicates that clustering stably converges using an iterative training scheme.

In Chapter 4, we proposed a DCAE model capable of learning compact data representation that can be incorporated into different learning schemes, i.e. unsupervised, semi-supervised, supervised. Two regularization techniques were also considered: one that is embedded into the 
clustering layer and another imposed into the training process. To the best of our knowledge, our work provides the first analytical study seeking to establish an understanding of the effectiveness of the discriminatory power obtained by two discriminative attributes: data-driven discriminative attributes with the support of regularization techniques, and supervision discriminative attributes with the support of supervision knowledge. We evaluated our experimental models on MNIST, USPS, MNIST fashion, and SVHN datasets, and showed the clustering accuracy of our framework through supervised, semi-supervised and unsupervised learning levels. The experimental results illustrated the influence of discriminatory power on clustering performance, and it was found that such supervision knowledge greatly helps to form discriminative transformations that are learned by the encoding part of the DCAE model, significantly improving the performance of clustering. The results also demonstrated that even weak or partial supervision knowledge could significantly improve the quality of deep clustering. Finally, the impact of the regularization techniques through a certain level of supervision has been discussed, showing that GBAR efficiently benefits the performance of semi-supervised clustering.

Chapter 5 proposed an iterative pruning method that prunes neurons based on their level of importance during training. We introduced a majority voting technique to assign a voting score when evaluating neurons' importance, alternately identifying the most critical neurons and removing redundancy accordingly. The effectiveness of our importance method becomes apparent when compared with several baselines. We empirically evaluated the proposed method across various scenarios, including fully-connected networks (FCNs), sparsely-connected networks (SCNs), and convolutional neural networks (CNNs) using the MNIST and CIFAR-10 datasets. The experimental results demonstrated the effectiveness of our pruning method in maintaining or even improving accuracy after removing unimportant neurons. We illustrated how our proposed method can reduce network parameters by up to $90 \%$ with no significant loss in the model's accuracy. The results also demonstrated that our proposed method is applicable to weight-based pruning methods and adds extra compression. Moreover, we showed that sparse models can be further pruned into even smaller models with our proposed method while preserving reference model accuracy.

Chapter 6 detailed our novel framework based on an effective channel-level pruning method, considering the power of novel neural network interpretability in evaluating the importance of feature maps. Based on the discriminative ability interpretable latent representations, a majority voting technique was proposed to compare the degree of alignment values among 
filters and assign a voting score to quantitatively evaluate the importance of feature maps. The experimental results showed the effectiveness of our filter selection criteria, which outperforms state-of-the-art pruning criteria and allows for the identification of layers which are robust or sensitive to pruning. We also proposed a simple but effective method to estimate new convolution kernels based on the remaining crucial channels to accomplish effective CNN compression. The experimental results on CIFAR-10, CUB-200, and ImageNet (ILSVRC 2012) datasets demonstrated the effectiveness of our pruning framework in maintaining and improving accuracy after removing unimportant filters, highlighting the excellent performance of our proposed method. We also compared our approach with other state-of-the-art pruning methods and showed a similar reduction in FLOPs with comparable or greater accuracy. Our pruned model can be further pruned into even smaller models by adopting any other model compression method.

Chapter 7 comprised a comprehensive review of conventional time-series data analysis, focusing on the time-series data, several choices of similarity measures and feature extraction, and popular conventional clustering algorithms. We then presented a founding contribution to the area of applying deep clustering to time-series data by providing the first case study in the context of movement behavior clustering, utilizing the DeepCluster method to cluster animal behavior through movement. The results were promising, showing that the latent space has encoded sufficient patterns and facilitated better clustering of movement behaviors. Subsequently, we reviewed other works that have been published since the publication of our work, identifing the recent state-of-the-art and presenting an outlook on the important field of deep time-series clustering from five important perspectives. This chapter is intended to be a keystone for future research on DTSC.

\subsection{Future Work}

Adapting deep clustering approaches, which alternately learn feature representation and cluster assignments, to a more generalized form provides enormous scope, as embedding a clustering approach into deep networks may benefit other application domains. By generalizing the usability of the latent space, it becomes possible to enable more applications to access the significant potential gains. We believe the latent space with clustering cooperation has the potential to be used for diverse tasks such as image generation/ synthesis. The latent space of the deep clustering method not only captures representative features and the relationships among repetitive patterns, but also aids in finding interesting anomalies for a variety of applications. The 
number of application domains is vast and stretches to fields as diverse as biology, medicine, finance, and animation. Research is therefore recommended to explore how such applications are able to make use of deep clustering methods, especially with a shortage of labeled data.

The development of methodologies within the field of deep clustering is still part of a relatively young and emerging field. Most of the proposed methods rely on the encoder's capabilities, as the clustering target distributions are estimated based on the learned representations in the latent space. As a result, focusing on strengthening the discriminative features and improving the encoder's ability is an important direction for future work. Chapter 4 provided an analytical study that establishes efficient discriminatory power. Similarly, it is possible to introduce more effective regularizations or tricks through the learning process, and research should be carried out into how such regularizations could benefit the achievement of deep clustering. This procedure opens up potential research directions with regards to imposing further constraints in the encoding part and strengthening the discriminative features learned by the encoder for DeepCluster. Although this procedure significantly boosts clustering performance, the more theoretical analysis also requires further study to understand how to improve clustering performance further. Moreover, simultaneous optimization of the objective function for deep clustering may present an effective solution to improve clustering results, allowing to simultaneously optimize the parameters of the network and clustering.

The prior-known number of clusters is still an area of active study, and making use of deep clustering will help to develop an understanding of the choice of approaches for a given DeepCluster architecture and, thus, for designing the best deep clustering architecture. In DeepCluster, the number of nodes in the clustering layer (encoding layer) always depends on the task at hand, i.e. the predefined number of clustering. Although this setting was applicable in practice, we believe more investigation is needed to gather conclusive evidence, carefully identify the best architecture choices for DeepCluster, and explore the usage of the model to find the number of clusters without any label information.

Although existing deep clustering methods achieved remarkable accuracy on MNIST and USPS, which are the most commonly used datasets in deep clustering, such methods demonstrate limited performance when dealing with more complex datasets, as their common structures are not well-formed. For instance, large appearance variance and image noise are common in a dataset like SVHN. It has been observed that the DeepCluster method grouped the SVHN's data samples based on the shape and color of their backgrounds. By evaluating the importance of filters and discarding the unnecessary parts of neural networks, as discussed 
in Chapter 6, it would be possible to force the learned features to be more discriminative. We have shown that identifying feature maps that focus on foreground objects rather than the background would determine individual filters to deliver essential information with strong discriminative features. Thus, developing deep clustering inspired by neural network interpretability and network compression is another potential research direction.

The effectiveness of deep representations has been shown to extend to network pruning. Our pruning methods presented in this study make use of quantifying the importance of latent representations. In Chapter 6, our approach compresses and accelerates CNNs for image classification tasks, including CIFAR object recognition, CUB-200 fine-grained classification, and ImageNet large-scale object classification. Applying our pruning method to real applications in several different computer vision tasks, including object detection, semantic segmentation, image generation, image retrieval, and style transfer is a fertile avenue for future research, as these visual tasks require richer knowledge and more abstract feature representation than image classification, meaning that they may face a sharp reduction in model performance $[454,455]$. Research could visually explore how such applications are capable of making use of our pruning method, particularly semantic segmentation and image generation.

Our proposed CNN compression and acceleration approach mainly focuses on filter-level pruning, where removing the unimportant filter in its entirety does not affect the network structure. This would allow for greater compression and acceleration by other compression approaches, such as the parameter quantization approach, and low-rank factorization methods. Although these approaches are computationally expensive and cannot perform global parameter compression, integrating them with ours would obtain more compressed networks for further improvement. It would also be fruitful to explore the usage of a hybrid scheme for network compression, where the advantages of each network compression category can be exploited to prune models further.

There are also several challenges and extensions we perceive as useful research directions. The first would be to extend our proposed framework and combine it with an iterative pruning method to more deeply explore the problem and accomplish effective CNN compression and acceleration, as pruning a network via a training process may provide more effective solutions. Secondly, most network interpretability methods are data-driven based, so their speed efficiency is a significant concern. Although pruning-based methods inspired by neural network interpretability achieved better results, it can be time-consuming to complete their process. Although a few images are selected from each category to form our evaluation set used to find the 
optimal channel subset, our proposed method still requires more than seven minutes to estimate IoU scores and MV values for one block only on ResNet-50 and ImageNet. Therefore, parallel implementation could be a promising solution, where CNN-based methods are more suitable for efficient parallelization benefit on both CPUs and GPUs. Consideration of a set of nodes, filters, and layers for pruning, instead of one by one in a greedy manner is also worthwhile to study in our future work.

Overall, the potential for deep network compression is vast; the field has many open problems to understand and explore. The remarkable advancement of neural network interpretability should encourage the development of efficient methods for network compression and acceleration to facilitate the deployment of advanced deep networks. 


\section{Bibliography}

[1] A. Alqahtani, X. Xie, M. W. Jones, and E. Essa, "Pruning cnn filters via quantifying the importance of deep visual representations," Computer Vision and Image Understanding, 2020, submitted.

[2] A. Alqahtani, X. Xie, E. Essa, and M. W. Jones, "Neuron-based network pruning based on majority voting," in Proceedings of the International Conference on Pattern Recognition, 2020, pp. 3090-3097.

[3] M. Ali, A. Alqahtani, M. W. Jones, and X. Xie, "Clustering and classification for time series data in visual analytics: A survey," IEEE Access, vol. 7, pp. 181314-181338, 2019.

[4] A. Alqahtani, X. Xie, J. Deng, and M. W. Jones, "Learning discriminatory deep clustering models," in Proceedings of the International Conference on Computer Analysis of Images and Patterns, 2019, pp. 224-233.

[5] A. Alqahtani, X. Xie, J. Deng, and M. Jones, "A deep convolutional auto-encoder with embedded clustering," in Proceedings of the IEEE International Conference on Image Processing, 2018, pp. 4058-4062.

[6] A. Zhang, Z. C. Lipton, M. Li, and A. J. Smola, Dive into Deep Learning, 2020. [Online]. Available: https://d2l.ai

[7] R. Girshick, "Fast r-cnn," in Proceedings of the IEEE International Conference on Computer Vision, 2015, pp. 1440-1448.

[8] H. Noh, S. Hong, and B. Han, "Learning deconvolution network for semantic segmentation," in Proceedings of the IEEE Conference on Computer Vision and Pattern Recognition, 2015, pp. 1520-1528. 
[9] A. Krizhevsky, I. Sutskever, and G. E. Hinton, "Imagenet classification with deep convolutional neural networks," in Proceedings of the Advances in Neural Information Processing Systems, 2012, pp. 1097-1105.

[10] K. Simonyan and A. Zisserman, "Very deep convolutional networks for large-scale image recognition," in Proceedings of the International Conference on Learning Representations, 2015.

[11] K. He, X. Zhang, S. Ren, and J. Sun, "Deep residual learning for image recognition," in Proceedings of the IEEE Conference on Computer Vision and Pattern Recognition, 2016, pp. 770-778.

[12] Y. Bengio, A. Courville, and P. Vincent, "Representation learning: A review and new perspectives," IEEE Transactions on Pattern Analysis and Machine Intelligence, vol. 35, no. 8, pp. 1798-1828, 2013.

[13] G. C. Nutakki, B. Abdollahi, W. Sun, and O. Nasraoui, "An introduction to deep clustering," in Clustering Methods for Big Data Analytics, 2019, pp. 73-89.

[14] R. Xu and D. Wunsch, "Survey of clustering algorithms," IEEE Transactions on Neural Networks, vol. 16, no. 3, pp. 645-678, 2005.

[15] D. Xu and Y. Tian, "A comprehensive survey of clustering algorithms," Annals of Data Science, vol. 2, no. 2, pp. 165-193, 2015.

[16] P. Berkhin, "A survey of clustering data mining techniques," in Grouping Multidimensional Bata. Springer, 2006, pp. 25-71.

[17] S. Wold, K. Esbensen, and P. Geladi, "Principal component analysis," Chemometrics and Intelligent Laboratory Systems, vol. 2, no. 1-3, pp. 37-52, 1987.

[18] T. Hofmann, B. Schölkopf, and A. J. Smola, "Kernel methods in machine learning," The Annals of Statistics, pp. 1171-1220, 2008.

[19] A. Y. Ng, M. I. Jordan, and Y. Weiss, "On spectral clustering: Analysis and an algorithm," in Proceedings of the Advances in Neural Information Processing Systems, 2002, pp. 849-856. 
[20] E. Min, X. Guo, Q. Liu, G. Zhang, J. Cui, and J. Long, "A survey of clustering with deep learning: From the perspective of network architecture," IEEE Access, vol. 6, pp. 39 501-39514, 2018.

[21] Y. LeCun, Y. Bengio, and G. Hinton, "Deep learning," Nature, vol. 521, no. 7553, pp. 436-444, 2015.

[22] E. L. Denton, W. Zaremba, J. Bruna, Y. LeCun, and R. Fergus, "Exploiting linear structure within convolutional networks for efficient evaluation," in Proceedings of the Advances in Neural Information Processing Systems, 2014, pp. 1269-1277.

[23] W. Wen, C. Wu, Y. Wang, Y. Chen, and H. Li, "Learning structured sparsity in deep neural networks," in Proceedings of the Advances in Neural Information Processing Systems, 2016, pp. 2074-2082.

[24] Y. Cheng, D. Wang, P. Zhou, and T. Zhang, "Model compression and acceleration for deep neural networks: The principles, progress, and challenges," IEEE Signal Processing Magazine, vol. 35, no. 1, pp. 126-136, 2018.

[25] R. R. Selvaraju, M. Cogswell, A. Das, R. Vedantam, D. Parikh, and D. Batra, "Gradcam: Visual explanations from deep networks via gradient-based localization," in Proceedings of the IEEE International Conference on Computer Vision, 2017, pp. 618-626.

[26] J.-Y. Zhu, P. Krähenbühl, E. Shechtman, and A. A. Efros, "Generative visual manipulation on the natural image manifold," in Proceedings of the European Conference on Computer Vision, 2016, pp. 597-613.

[27] B. Zhou, Y. Sun, D. Bau, and A. Torralba, "Interpretable basis decomposition for visual explanation," in Proceedings of the European Conference on Computer Vision, 2018, pp. 119-134.

[28] T. Hastie, R. Tibshirani, and J. Friedman, The elements of statistical learning: data mining, inference, and prediction. Springer, 2009.

[29] C. M. Bishop, Pattern recognition and machine learning. Springer, 2006.

[30] V. Vo, J. Luo, and B. Vo, "Time series trend analysis based on k-means and support vector machine," Computing and Informatics, vol. 35, pp. 111-127, 2016. 
[31] E. Alpaydin, Introduction to machine learning. MIT press, 2009.

[32] I. Goodfellow, Y. Bengio, and A. Courville, Deep Learning. MIT Press, 2016. [Online]. Available: https://www.deeplearningbook.org

[33] O. Simeone, "A very brief introduction to machine learning with applications to communication systems," IEEE Transactions on Cognitive Communications and Networking, vol. 4, no. 4, pp. 648-664, 2018.

[34] N. Dalal and B. Triggs, "Histograms of oriented gradients for human detection," in Proceedings of the IEEE Conference on Computer Vision and Pattern Recognition, 2005, pp. 886-893.

[35] D. G. Lowe, "Object recognition from local scale-invariant features," in Proceedings of the IEEE Conference on Computer Vision and Pattern Recognition, 1999, pp. 11501157.

[36] F. Rosenblatt, "The perceptron: a probabilistic model for information storage and organization in the brain." Psychological Review, vol. 65, no. 6, p. 386, 1958.

[37] H. B. Demuth, M. H. Beale, O. De Jess, and M. T. Hagan, Neural network design. Martin Hagan, 2014.

[38] W. S. McCulloch and W. Pitts, "A logical calculus of the ideas immanent in nervous activity," The Bulletin of Mathematical Biophysics, vol. 5, no. 4, pp. 115-133, 1943.

[39] F. Rosenblatt and S. Papert, "The perceptron," A Perceiving and Recognizing Automation, Cornell Aeronautical Laboratory Report, pp. 85-460, 1957.

[40] P. Dole, Neuroscience. Oxford University Press, 2012.

[41] A. Nanopoulos, R. Alcock, and Y. Manolopoulos, "Feature-based classification of timeseries data," International Journal of Computer Research, vol. 10, no. 3, pp. 49-61, 2001.

[42] C. Szegedy, W. Liu, Y. Jia, P. Sermanet, S. Reed, D. Anguelov, D. Erhan, V. Vanhoucke, and A. Rabinovich, "Going deeper with convolutions," in Proceedings of the IEEE Conference on Computer Vision and Pattern Recognition, 2015, pp. 1-9. 
[43] J. Frankle and M. Carbin, "The lottery ticket hypothesis: Finding sparse, trainable neural networks," in Proceedings of the International Conference on Learning Representations, 2019.

[44] S. Arora, R. Ge, B. Neyshabur, and Y. Zhang, "Stronger generalization bounds for deep nets via a compression approach," in Proceedings of the International Conference on Machine Learning, 2018, pp. 254-263.

[45] A. S. Morcos, D. G. Barrett, N. C. Rabinowitz, and M. Botvinick, "On the importance of single directions for generalization," in Proceedings of the International Conference on Learning Representations, 2018.

[46] K. O. Stanley and R. Miikkulainen, "Evolving neural networks through augmenting topologies," Evolutionary Computation, vol. 10, no. 2, pp. 99-127, 2002.

[47] K. O. Stanley, D. B. D’ Ambrosio, and J. Gauci, "A hypercube-based encoding for evolving large-scale neural networks," Artificial Life, vol. 15, no. 2, pp. 185-212, 2009.

[48] P. Molchanov, S. Tyree, T. Karras, T. Aila, and J. Kautz, "Pruning convolutional neural networks for resource efficient inference," in Proceedings of the International Conference on Learning Representations, 2017.

[49] D. E. Rumelhart, G. E. Hinton, and R. J. Williams, "Learning representations by backpropagating errors," Nature, vol. 323, no. 6088, pp. 533-536, 1986.

[50] K. Janocha and W. M. Czarnecki, "On loss functions for deep neural networks in classification," CoRR, vol. abs/1702.05659, 2017.

[51] A. G. Baydin, B. A. Pearlmutter, A. A. Radul, and J. M. Siskind, "Automatic differentiation in machine learning: a survey," Journal of Machine Learning Research, vol. 18, no. 1, pp. 5595-5637, 2017.

[52] M. Abadi, P. Barham, J. Chen, Z. Chen, A. Davis, J. Dean, M. Devin, S. Ghemawat, G. Irving, M. Isard et al., "Tensorflow: A system for large-scale machine learning," in Proceedings of the Symposium on Operating Systems Design and Implementation, 2016, pp. 265-283. 
[53] R. Pascanu, T. Mikolov, and Y. Bengio, "On the difficulty of training recurrent neural networks," in Proceedings of the International Conference on Machine Learning, 2013, pp. 1310-1318.

[54] S. Hochreiter and J. Schmidhuber, "Long short-term memory," Neural Computation, vol. 9, no. 8, pp. 1735-1780, 1997.

[55] S. Ioffe and C. Szegedy, "Batch normalization: Accelerating deep network training by reducing internal covariate shift," in Proceedings of the International Conference on Machine Learning, 2015, pp. 448-456.

[56] N. Srivastava, G. Hinton, A. Krizhevsky, I. Sutskever, and R. Salakhutdinov, "Dropout: a simple way to prevent neural networks from overfitting," Journal of Machine Learning Research, vol. 15, no. 1, pp. 1929-1958, 2014.

[57] X. Glorot and Y. Bengio, "Understanding the difficulty of training deep feedforward neural networks," in Proceedings of the International Conference on Artificial Intelligence and Statistics, 2010, pp. 249-256.

[58] K. He, X. Zhang, S. Ren, and J. Sun, "Delving deep into rectifiers: Surpassing humanlevel performance on imagenet classification," in Proceedings of the IEEE Conference on Computer Vision and Pattern Recognition, 2015, pp. 1026-1034.

[59] Y. Bengio, "Practical recommendations for gradient-based training of deep architectures," in Neural Networks: Tricks of the Trade. Springer, 2012, pp. 437-478.

[60] M. Li, T. Zhang, Y. Chen, and A. J. Smola, "Efficient mini-batch training for stochastic optimization," in Proceedings of the International Conference on Knowledge Discovery and Data Mining, 2014, pp. 661-670.

[61] J. Duchi, E. Hazan, and Y. Singer, "Adaptive subgradient methods for online learning and stochastic optimization." Journal of Machine Learning Research, p. 2121-2159, 2011.

[62] M. D. Zeiler, “Adadelta: an adaptive learning rate method," CoRR, vol. abs/1212.5701, 2012.

[63] D. P. Kingma and J. Ba, "Adam: A method for stochastic optimization," in Proceedings of the International Conference on Learning Representations, 2015. 
[64] G. B. Goh, N. O. Hodas, and A. Vishnu, "Deep learning for computational chemistry," Journal of Computational Chemistry, pp. 1291-1307, 2017.

[65] V. Nair and G. E. Hinton, "Rectified linear units improve restricted Boltzmann machines," in Proceedings of the International Conference on Machine Learning, 2010, p. 807-814.

[66] B. Xu, N. Wang, T. Chen, and M. Li, "Empirical evaluation of rectified activations in convolutional network," CoRR, vol. abs/1505.00853, 2015.

[67] K. Jarrett, K. Kavukcuoglu, M. Ranzato, and Y. LeCun, "What is the best multi-stage architecture for object recognition?" in Proceedings of the IEEE International Conference on Computer Vision, 2009, pp. 2146-2153.

[68] Y. LeCun, L. Bottou, Y. Bengio, P. Haffner et al., "Gradient-based learning applied to document recognition," Proceedings of the IEEE, vol. 86, no. 11, pp. 2278-2324, 1998.

[69] Y. Zheng, Q. Liu, E. Chen, Y. Ge, and J. L. Zhao, "Time series classification using multi-channels deep convolutional neural networks," in Proceedings of the International Conference on Web-Age Information Management, 2014, pp. 298-310.

[70] M. D. Zeiler and R. Fergus, "Visualizing and understanding convolutional networks," in Proceedings of the European Conference on Computer Vision, 2014, pp. 818-833.

[71] Y.-L. Boureau, J. Ponce, and Y. LeCun, "A theoretical analysis of feature pooling in visual recognition," in Proceedings of the International Conference on Machine Learning, 2010, pp. 111-118.

[72] J. Deng, W. Dong, R. Socher, L.-J. Li, K. Li, and L. Fei-Fei, "Imagenet: A largescale hierarchical image database," in Proceedings of the IEEE Conference on Computer Vision and Pattern Recognition, 2009, pp. 248-255.

[73] R. Girshick, J. Donahue, T. Darrell, and J. Malik, "Rich feature hierarchies for accurate object detection and semantic segmentation," in Proceedings of the IEEE Conference on Computer Vision and Pattern Recognition, 2014, pp. 580-587.

[74] S. Ren, K. He, R. Girshick, and J. Sun, "Faster R-CNN: towards real-time object detection with region proposal networks," IEEE Transactions on Pattern Analysis and Machine Intelligence, vol. 39, no. 6, pp. 1137-1149, 2016. 
[75] J. Dai, Y. Li, K. He, and J. Sun, "R-FCN: Object detection via region-based fully convolutional networks," in Proceedings of the Advances in Neural Information Processing Systems, 2016, pp. 379-387.

[76] T.-Y. Lin, P. Dollar, R. Girshick, K. He, B. Hariharan, and S. Belongie, "Feature pyramid networks for object detection," in Proceedings of the IEEE Conference on Computer Vision and Pattern Recognition, 2017, pp. 2117-2125.

[77] K. He, G. Gkioxari, P. Dollar, and R. Girshick, "Mask R-CNN," in Proceedings of the IEEE International Conference on Computer Vision, 2017, pp. 2961-2969.

[78] J. Long, E. Shelhamer, and T. Darrell, "Fully convolutional networks for semantic segmentation," in Proceedings of the IEEE International Conference on Computer Vision, 2015, pp. 3431-3440.

[79] H. Zhao, J. Shi, X. Qi, X. Wang, and J. Jia, "Pyramid scene parsing network," in Proceedings of the IEEE Conference on Computer Vision and Pattern Recognition, 2017, pp. 2881-2890.

[80] J. R. Uijlings, K. E. Van De Sande, T. Gevers, and A. W. Smeulders, "Selective search for object recognition," International Journal of Computer Vision, vol. 104, no. 2, pp. 154-171, 2013.

[81] G. Papandreou, L.-C. Chen, K. P. Murphy, and A. L. Yuille, "Weakly-and semisupervised learning of a deep convolutional network for semantic image segmentation," in Proceedings of the IEEE International Conference on Computer Vision, 2015, pp. 1742-1750.

[82] J. T. Barron, "A general and adaptive robust loss function," in Proceedings of the IEEE Conference on Computer Vision and Pattern Recognition, 2019, pp. 4331-4339.

[83] Y. Lecun, L. Bottou, Y. Bengio, and P. Haffner, "Gradient-based learning applied to document recognition," Proceedings of the IEEE, vol. 86, no. 11, pp. 2278-2324, Nov 1998.

[84] Y. Wang, Z. Xie, K. Xu, Y. Dou, and Y. Lei, "An efficient and effective convolutional auto-encoder extreme learning machine network for 3d feature learning," Neurocomputing, vol. 174, pp. 988-998, 2016. 
[85] J. Masci, U. Meier, D. Ciresan, and J. Schmidhuber, "Stacked convolutional autoencoders for hierarchical feature extraction," in Proceedings of the International Conference on Artificial Neural Networks, 2011, pp. 52-59.

[86] W. Shi, J. Caballero, L. Theis, F. Huszar, A. Aitken, C. Ledig, and Z. Wang, "Is the deconvolution layer the same as a convolutional layer?" CoRR, vol. abs/1609.07009, 2016.

[87] M. D. Zeiler, G. W. Taylor, and R. Fergus, "Adaptive deconvolutional networks for mid and high level feature learning," in Proceedings of the IEEE International Conference on Computer Vision, 2011, pp. 2018-2025.

[88] M. D. Zeiler, D. Krishnan, G. W. Taylor, and R. Fergus, "Deconvolutional networks," in Proceedings of the IEEE Conference on Computer Vision and Pattern Recognition. IEEE, 2010, pp. 2528-2535.

[89] I. Goodfellow, J. Pouget-Abadie, M. Mirza, B. Xu, D. Warde-Farley, S. Ozair, A. Courville, and Y. Bengio, "Generative adversarial nets," in Proceedings of the Advances in Neural Information Processing Systems, 2014, pp. 2672-2680.

[90] A. Radford, L. Metz, and S. Chintala, "Unsupervised representation learning with deep convolutional generative adversarial networks," CoRR, vol. abs/1511.06434, 2015.

[91] T. Salimans, I. Goodfellow, W. Zaremba, V. Cheung, A. Radford, and X. Chen, "Improved techniques for training gans," in Proceedings of the Advances in Neural Information Processing Systems, 2016, pp. 2234-2242.

[92] X. Chen, Y. Duan, R. Houthooft, J. Schulman, I. Sutskever, and P. Abbeel, "Infogan: Interpretable representation learning by information maximizing generative adversarial nets," in Proceedings of the Advances in Neural Information Processing Systems, 2016, pp. 2180-2188.

[93] A. Graves, A.-r. Mohamed, and G. Hinton, "Speech recognition with deep recurrent neural networks," in Proceedings of IEEE International Conference on Acoustics, Speech and Signal Processing, 2013, pp. 6645-6649.

[94] Y. Wu, M. Schuster, Z. Chen, Q. V. Le, M. Norouzi, W. Macherey, M. Krikun, Y. Cao, Q. Gao, K. Macherey et al., "Google's neural machine translation system: Bridging the gap between human and machine translation," CoRR, vol. abs/1609.08144, 2016. 
[95] A. Dosovitskiy and T. Brox, "Inverting visual representations with convolutional networks," in Proceedings of the IEEE Conference on Computer Vision and Pattern Recognition, 2016, pp. 4829-4837.

[96] A. Mahendran and A. Vedaldi, "Understanding deep image representations by inverting them," in Proceedings of the IEEE Conference on Computer Vision and Pattern Recognition, 2015, pp. 5188-5196.

[97] D. Erhan, Y. Bengio, A. Courville, and P. Vincent, "Visualizing higher-layer features of a deep network," Technical report, University of Montreal, vol. 1341, no. 3, 2009. [Online]. Available: http://www.iro.umontreal.ca/ lisa/publications2/index.php/ publications/show/247

[98] K. Simonyan, A. Vedaldi, and A. Zisserman, "Deep inside convolutional networks: Visualising image classification models and saliency maps," CoRR, vol. abs/1312.6034, 2013.

[99] J. Yosinski, J. Clune, A. Nguyen, T. Fuchs, and H. Lipson, "Understanding neural networks through deep visualization," CoRR, vol. abs/1506.06579, 2015.

[100] D. Bau, B. Zhou, A. Khosla, A. Oliva, and A. Torralba, "Network dissection: Quantifying interpretability of deep visual representations," in Proceedings of the IEEE Conference on Computer Vision and Pattern Recognition, 2017, pp. 6541-6549.

[101] D. Bau, J.-Y. Zhu, H. Strobelt, B. Zhou, J. B. Tenenbaum, W. T. Freeman, and A. Torralba, "Gan dissection: Visualizing and understanding generative adversarial networks," in Proceedings of the International Conference on Learning Representations, 2019.

[102] K. Dhamdhere, M. Sundararajan, and Q. Yan, "How important is a neuron?" in Proceedings of the International Conference on Learning Representations, 2019.

[103] S. Na, Y. J. Choe, D.-H. Lee, and G. Kim, "Discovery of natural language concepts in individual units of cnns," in Proceedings of the International Conference on Learning Representations, 2019.

[104] S. Bach, A. Binder, G. Montavon, F. Klauschen, K.-R. Muller, and W. Samek, "On pixel-wise explanations for non-linear classifier decisions by layer-wise relevance propagation," PloS one, vol. 10, no. 7, 2015. 
[105] M. Liu, J. Shi, Z. Li, C. Li, J. Zhu, and S. Liu, "Towards better analysis of deep convolutional neural networks," IEEE Transactions on Visualization and Computer Graphics, vol. 23, no. 1, pp. 91-100, 2017.

[106] F.-Y. Tzeng and K.-L. Ma, "Opening the black box-data driven visualization of neural networks," in Proceedings of the IEEE Visualization, 2005, pp. 383-390.

[107] R. Guidotti, A. Monreale, S. Ruggieri, F. Turini, F. Giannotti, and D. Pedreschi, "A survey of methods for explaining black box models," ACM Computing Surveys (CSUR), pp. 1-42, 2018.

[108] G. Montavon, W. Samek, and K.-R. Muller, "Methods for interpreting and understanding deep neural networks," Digital Signal Processing, vol. 73, pp. 1-15, 2018.

[109] W. Samek, G. Montavon, S. Lapuschkin, C. J. Anders, and K.-R. Muller, "Explaining deep neural networks and beyond: A review of methods and applications," Proceedings of the IEEE, vol. 109, no. 3, pp. 247-278, 2021.

[110] G. Montavon, A. Binder, S. Lapuschkin, W. Samek, and K.-R. Muller, "Layer-wise relevance propagation: an overview," Explainable AI: Interpreting, Explaining and Visualizing Deep Learning, pp. 193-209, 2019.

[111] G. Montavon, S. Lapuschkin, A. Binder, W. Samek, and K.-R. Muller, "Explaining nonlinear classification decisions with deep taylor decomposition," Pattern Recognition, vol. 65, pp. 211-222, 2017.

[112] L. Arras, J. Arjona-Medina, M. Widrich, G. Montavon, M. Gillhofer, K.-R. Muller, S. Hochreiter, and W. Samek, "Explaining and interpreting LSTMs," in Explainable AI: Interpreting, Explaining and Visualizing Deep Learning, 2019, pp. 211-238.

[113] B. Zhou, D. Bau, A. Oliva, and A. Torralba, "Interpreting deep visual representations via network dissection," IEEE Transactions on Pattern Analysis and Machine Intelligence, vol. 41, no. 9, pp. 2131-2145, 2018.

[114] D. Bau, J.-Y. Zhu, H. Strobelt, A. Lapedriza, B. Zhou, and A. Torralba, "Understanding the role of individual units in a deep neural network," Proceedings of the National Academy of Sciences, vol. 117, no. 48, pp. 30 071-30 078, 2020. 
[115] B. Kim, M. Wattenberg, J. Gilmer, C. Cai, J. Wexler, F. Viegas et al., "Interpretability beyond feature attribution: Quantitative testing with concept activation vectors (TCAV)," in Proceedings of the International Conference on Machine Learning, 2018, pp. 2668 2677.

[116] M. Simon, E. Rodner, T. Darrell, and J. Denzler, "The whole is more than its parts? from explicit to implicit pose normalization," IEEE Transactions on Pattern Analysis and Machine Intelligence, vol. 42, no. 3, pp. 749-763, 2018.

[117] Q. Zhang, X. Wang, R. Cao, Y. N. Wu, F. Shi, and S.-C. Zhu, "Extracting an explanatory graph to interpret a CNN," IEEE Transactions on Pattern Analysis and Machine Intelligence, 2020.

[118] L. A. Hendricks, Z. Akata, M. Rohrbach, J. Donahue, B. Schiele, and T. Darrell, "Generating visual explanations," in Proceedings of the European Conference on Computer Vision, 2016, pp. 3-19.

[119] R. A. Amjad, K. Liu, and B. C. Geiger, "Understanding individual neuron importance using information theory," CoRR, vol. abs/1804.06679, 2018.

[120] L. Kaufman and P. J. Rousseeuw, Finding groups in data: an introduction to cluster analysis. John Wiley \& Sons, 2009, vol. 344.

[121] J. MacQueen et al., "Some methods for classification and analysis of multivariate observations," in Proceedings of the Berkeley Symposium on Mathematical Statistics and Probability, 1967, pp. 281-297.

[122] L. Rokach and O. Maimon, "Clustering methods," in Data Mining and Knowledge Discovery Handbook. Springer, 2005, pp. 321-352.

[123] C. Goutte, P. Toft, E. Rostrup, F. A. Nielsen, and L. K. Hansen, "On clustering fMRI time series," NeuroImage, vol. 9, no. 3, pp. 298-310, 1999.

[124] H. Ziegler, M. Jenny, T. Gruse, and D. A. Keim, "Visual market sector analysis for financial time series data," in Proceedings of the IEEE Symposium on Visual Analytics Science and Technology, 2010, pp. 83-90.

[125] F. Klawonn, "Fuzzy clustering: insights and a new approach," Mathware \& Soft Computing, vol. 11, 2004. 
[126] J. C. Dunn, A fuzzy relative of the ISODATA process and its use in detecting compact well-separated clusters. Taylor \& Francis, 1973.

[127] J. C. Bezdek, "Pattern recognition with fuzzy objective function algorithms," in Proceedings of the Advanced Applications in Pattern Recognition, 1981.

[128] S. Eschrich, J. Ke, L. O. Hall, and D. B. Goldgof, "Fast accurate fuzzy clustering through data reduction," IEEE Transactions on Fuzzy Systems, vol. 11, no. 2, pp. 262-270, 2003.

[129] C. S. Möller-Levet, F. Klawonn, K.-H. Cho, and O. Wolkenhauer, "Fuzzy clustering of short time-series and unevenly distributed sam-pling points," in Proceedings of the International Symposium on Intelligent Data Analysis, 2003, pp. 330-340.

[130] J. C. Bezdek, "A convergence theorem for the fuzzy isodata clustering algorithms." IEEE Transactions on Pattern Analysis and Machine Intelligence, vol. 2, no. 1, pp. 1-8, 1980.

[131] R. L. Cannon, J. V. Dave, and J. C. Bezdek, "Efficient implementation of the fuzzy c-means clustering algorithms," IEEE Transactions on Pattern Analysis and Machine Intelligence, no. 2, pp. 248-255, 1986.

[132] R. Krishnapuram, A. Joshi, O. Nasraoui, and L.-y. Yi, "Low-complexity fuzzy relational clustering algorithms for web mining," IEEE Transactions on Fuzzy Systems, vol. 9, no. 4 , pp. 595-607, 2001.

[133] S. Das, A. Abraham, and A. Konar, "Automatic clustering using an improved differential evolution algorithm," IEEE Transactions on Systems, Man, and Cybernetics-Part A: Systems and Humans, vol. 38, no. 1, pp. 218-237, 2008.

[134] S. Aghabozorgi, A. S. Shirkhorshidi, and T. Y. Wah, "Time-series clustering-a decade review," Information Systems, vol. 53, pp. 16-38, 2015.

[135] J. Bernard, N. Wilhelm, B. Krüger, T. May, T. Schreck, and J. Kohlhammer, "Motionexplorer: Exploratory search in human motion capture data based on hierarchical aggregation," IEEE Transactions on Visualization and Computer Graphics, vol. 19, no. 12, pp. 2257-2266, 2013.

[136] E. Keogh and J. Lin, "Clustering of time-series subsequences is meaningless: implications for previous and future research," Knowledge and Information Systems, vol. 8, no. 2, pp. 154-177, 2005. 
[137] M. Halkidi, Y. Batistakis, and M. Vazirgiannis, "On clustering validation techniques," Journal of Intelligent Information Systems, vol. 17, no. 2-3, pp. 107-145, 2001.

[138] J. Lin, E. Keogh, S. Lonardi, and B. Chiu, "A symbolic representation of time series, with implications for streaming algorithms," in Proceedings of the Workshop on Research Issues in Data Mining and Knowledge Discovery, 2003, pp. 2-11.

[139] S. Guha, R. Rastogi, and K. Shim, "Cure: an efficient clustering algorithm for large databases," in ACM Sigmod Record, 1998, pp. 73-84.

[140] T. Zhang, R. Ramakrishnan, and M. Livny, "BIRCH: an efficient data clustering method for very large databases," in ACM Sigmod Record, vol. 25, no. 2, 1996, pp. 103-114.

[141] S. Aghabozorgi, M. R. Saybani, and T. Y. Wah, "Incremental clustering of time-series by fuzzy clustering," Journal of Information Science and Engineering, vol. 28, no. 4, pp. 671-688, 2012.

[142] K. Fukunaga and L. Hostetler, "The estimation of the gradient of a density function, with applications in pattern recognition," IEEE Transactions on Information Theory, vol. 21, no. 1 , pp. 32-40, 1975.

[143] D. A. Reynolds, "Gaussian mixture models.” Encyclopedia of Biometrics, vol. 741, 2009.

[144] T. Kohonen, “The self-organizing map," Proceedings of the IEEE, vol. 78, no. 9, pp. 1464-1480, 1990.

[145] M. Shanmuganathan, "An overview of IRFS on canadian GAAP - self-organizing maps (SOMs) MS," in SAI Computing Conference, 2016, pp. 100-107.

[146] V. J. Lobo, "Application of self-organizing maps to the maritime environment," in Information Fusion and Geographic Information Systems, 2009, pp. 19-36.

[147] F. Bação, V. Lobo, and M. Painho, "The self-organizing map, the Geo-SOM, and relevant variants for geosciences," Computers \& Geosciences, vol. 31, no. 2, pp. 155-163, 2005.

[148] M. Chang, H.-J. Yu, and J.-S. Heh, "Evolutionary self-organizing map," in Proceedings of the International Joint Conference on Neural Networks and IEEE World Congress on Computational Intelligence, vol. 1, 1998, pp. 680-685. 
[149] T. Kohonen, "The adaptive-subspace SOM (ASSOM) and its use for the implementation of invariant feature detection," in Proceedings of the International Conference on Artificial Neural Networks, vol. 95, 1995, pp. 3-10.

[150] T. Kohonen, "Emergence of invariant-feature detectors in the adaptive-subspace selforganizing map," Biological Cybernetics, vol. 75, no. 4, pp. 281-291, 1996.

[151] J. Walter and H. Ritter, "Rapid learning with parametrized self-organizing maps," Neurocomputing, vol. 12, no. 2, pp. 131-153, 1996.

[152] H. Yin, "Visualisation induced SOM (ViSOM)," in Proceedings of the Advances in SelfOrganising Maps, 2001, pp. 81-88.

[153] H. Yin, "ViSOM-a novel method for multivariate data projection and structure visualization," IEEE Transactions on Neural networks, vol. 13, no. 1, pp. 237-243, 2002.

[154] H. Yin and N. M. Allinson, "Self-organizing mixture networks for probability density estimation," IEEE Transactions on Neural Networks, vol. 12, no. 2, pp. 405-411, 2001.

[155] T. W. Liao, “Clustering of time series data - a survey,” Pattern Recognition, vol. 38, no. 11, pp. 1857-1874, 2005.

[156] H. Yin, "The self-organizing maps: background, theories, extensions and applications," in Computational Intelligence: A Compendium. Springer, 2008, pp. 715-762.

[157] X. Wang, K. A. Smith, R. Hyndman, and D. Alahakoon, "A scalable method for time series clustering," Research Paper, vol. 1, 2004. [Online]. Available: https: //citeseerx.ist.psu.edu/viewdoc/download?doi=10.1.1.155.207\&rep=rep1\&type=pdf

[158] M. Ester, H.-P. Kriegel, J. Sander, X. Xu et al., "A density-based algorithm for discovering clusters in large spatial databases with noise." in Proceedings of the International Conference on Knowledge Discovery and Data Mining, vol. 96, no. 34, 1996, pp. 226231.

[159] M. Ankerst, M. M. Breunig, H.-P. Kriegel, and J. Sander, "OPTICS: ordering points to identify the clustering structure," in ACM Sigmod Record, vol. 28, no. 2, 1999, pp. $49-60$.

[160] M. M. Breunig, H.-P. Kriegel, R. T. Ng, and J. Sander, "LOF: identifying density-based local outliers," in ACM Sigmod Record, vol. 29, no. 2, 2000, pp. 93-104. 
[161] X. Yang, L. J. Latecki, and D. Pokrajac, "Outlier detection with globally optimal exemplar-based GMM," in Proceedings of the SIAM International Conference on Data Mining, 2009, pp. 145-154.

[162] D. Cai, X. He, and J. Han, "Locally consistent concept factorization for document clustering," IEEE Transactions on Knowledge and Data Engineering, vol. 23, no. 6, pp. 902-913, 2010.

[163] R. Xu, "Measuring explained variation in linear mixed effects models," Statistics in Medicine, vol. 22, no. 22, pp. 3527-3541, 2003.

[164] D. D. Lee and H. S. Seung, "Learning the parts of objects by non-negative matrix factorization," Nature, vol. 401, no. 6755, pp. 788-791, 1999.

[165] A. Y. Yang, S. S. Sastry, A. Ganesh, and Y. Ma, "Fast 1-minimization algorithms and an application in robust face recognition: A review," in Proceedings of the IEEE International Conference on Image Processing, 2010, pp. 1849-1852.

[166] U. Von Luxburg, "A tutorial on spectral clustering," Statistics and Computing, vol. 17, no. 4, pp. 395-416, 2007.

[167] C. Song, F. Liu, Y. Huang, L. Wang, and T. Tan, "Auto-encoder based data clustering," in Proceedings of the Iberoamerican Congress on Pattern Recognition. Springer, 2013, pp. 117-124.

[168] F. Tian, B. Gao, Q. Cui, E. Chen, and T.-Y. Liu, "Learning deep representations for graph clustering." in Proceedings of the AAAI Conference on Artificial Intelligence, 2014, pp. $1293-1299$.

[169] P. Huang, Y. Huang, W. Wang, and L. Wang, "Deep embedding network for clustering," in Proceedings of the International Conference on Pattern Recognition, 2014, pp. 15321537.

[170] J. R. Hershey, Z. Chen, J. Le Roux, and S. Watanabe, "Deep clustering: Discriminative embeddings for segmentation and separation," in Proceedings of the IEEE International Conference on Acoustics, Speech and Signal Processing, 2016, pp. 31-35. 
[171] J. Xie, R. Girshick, and A. Farhadi, "Unsupervised deep embedding for clustering analysis," in Proceedings of the International Conference on Machine Learning, 2016, pp. 478-487.

[172] B. Yang, X. Fu, N. D. Sidiropoulos, and M. Hong, "Towards k-means-friendly spaces: Simultaneous deep learning and clustering," in Proceedings of the International Conference on Machine Learning, 2017, pp. 3861-3870.

[173] F. Schroff, D. Kalenichenko, and J. Philbin, "Facenet: A unified embedding for face recognition and clustering," in Proceedings of the IEEE Conference on Computer Vision and Pattern Recognition, 2015, pp. 815-823.

[174] F. Li, H. Qiao, B. Zhang, and X. Xi, "Discriminatively boosted image clustering with fully convolutional auto-encoders," Pattern Recognition, vol. 83, pp. 161-173, 2018.

[175] X. Guo, X. Liu, E. Zhu, and J. Yin, "Deep clustering with convolutional autoencoders," in Proceedings of the International Conference on Neural Information Processing, 2017, pp. 373-382.

[176] P. Vincent, H. Larochelle, Y. Bengio, and P. A. Manzagol, "Extracting and composing robust features with denoising autoencoders," in Proceedings of the International Conference on Machine Learning, 2008, pp. 1096-1103.

[177] G. E. Hinton, "A practical guide to training restricted Boltzmann machines," in Neural Networks: Tricks of the Trade, 2012, pp. 599-619.

[178] P. Baldi, "Autoencoders, unsupervised learning, and deep architectures," in Proceedings of the ICML Workshop on Unsupervised and Transfer Learning, 2012, pp. 37-49.

[179] P. Vincent, H. Larochelle, I. Lajoie, Y. Bengio, P.-A. Manzagol, and L. Bottou, "Stacked denoising autoencoders: Learning useful representations in a deep network with a local denoising criterion.” Journal of machine learning research, vol. 11, no. 12, 2010.

[180] G. E. Hinton and R. R. Salakhutdinov, "Reducing the dimensionality of data with neural networks," Science, vol. 313, no. 5786, pp. 504-507, 2006.

[181] S. Lloyd, "Least squares quantization in PCM," IEEE Transactions on Information Theory, vol. 28, no. 2, pp. 129-137, 1982. 
[182] V. M. Patel, H. Van Nguyen, and R. Vidal, "Latent space sparse subspace clustering," in Proceedings of the IEEE International Conference on Computer Vision, 2013, pp. $225-232$.

[183] J. Yang, D. Parikh, and D. Batra, "Joint unsupervised learning of deep representations and image clusters," in Proceedings of the IEEE Conference on Computer Vision and Pattern Recognition, 2016, pp. 5147-5156.

[184] X. Liu and W. B. Croft, "Cluster-based retrieval using language models," in Proceedings of the International Conference on Research and Development in Information Retrieval, 2004, pp. 186-193.

[185] E. Aljalbout, V. Golkov, Y. Siddiqui, M. Strobel, and D. Cremers, "Clustering with deep learning: Taxonomy and new methods," CoRR, vol. abs/1801.07648, 2018.

[186] K. Tian, S. Zhou, and J. Guan, "Deepcluster: a general clustering framework based on deep learning," in Proceedings of the Joint European Conference on Machine Learning and Knowledge Discovery in Databases, 2017, pp. 809-825.

[187] D. Cohn, R. Caruana, and A. McCallum, "Semi-supervised clustering with user feedback," Constrained Clustering: Advances in Algorithms, Theory, and Applications, vol. 4, no. 1, pp. 17-32, 2003.

[188] I. Davidson, S. Ravi, and M. Ester, "Efficient incremental constrained clustering," in Proceedings of the International Conference on Knowledge Discovery and Data Mining, 2007, pp. 240-249.

[189] A. Dubey, I. Bhattacharya, and S. Godbole, "A cluster-level semi-supervision model for interactive clustering," in Proceedings of the Joint European Conference on Machine Learning and Knowledge Discovery in Databases, 2010, pp. 409-424.

[190] H. P. Lai, M. Visani, A. Boucher, and J.-M. Ogier, "A new interactive semi-supervised clustering model for large image database indexing," Pattern Recognition Letters, vol. 37, pp. 94-106, 2014.

[191] S. Basu, A. Banerjee, and R. Mooney, "Semi-supervised clustering by seeding," in Proceedings of the International Conference on Machine Learning, 2002, pp. 19-26. 
[192] W. Pedrycz and J. Waletzky, "Fuzzy clustering with partial supervision," IEEE Transactions on Systems, Man, and Cybernetics, vol. 27, no. 5, pp. 787-795, 1997.

[193] K. Wagstaff, C. Cardie, S. Rogers, and S. Schrodl, "Constrained k-means clustering with background knowledge," in Proceedings of the International Conference on Machine Learning, 2001, pp. 577-584.

[194] S. Basu, A. Banerjee, and R. J. Mooney, "Active semi-supervision for pairwise constrained clustering," in Proceedings of the SIAM International Conference on Data Mining, 2004, pp. 333-344.

[195] I. Davidson and S. Ravi, "Agglomerative hierarchical clustering with constraints: Theoretical and empirical results," in Proceedings of the European Conference on Principles of Data Mining and Knowledge Discovery, 2005, pp. 59-70.

[196] C. Ruiz, M. Spiliopoulou, and E. Menasalvas, "Density-based semi-supervised clustering," Data mining and Knowledge Discovery, vol. 21, no. 3, pp. 345-370, 2010.

[197] A. Zaghian and F. Noorbehbahani, "A novel supervised cluster adjustment method using a fast exact nearest neighbor search algorithm," Pattern Analysis and Applications, vol. 20, no. 3, pp. 701-715, 2017.

[198] W. Pedrycz and G. Vukovich, "Fuzzy clustering with supervision," Pattern Recognition, vol. 37, no. 7, pp. 1339-1349, 2004.

[199] R. Tagliaferri, A. Staiano, and D. Scala, "A supervised fuzzy clustering for radial basis function neural networks training," in Proceedings of the Joint IFSA World Congress and NAFIPS International Conference, vol. 3, 2001, pp. 1804-1809.

[200] C. F. Eick, N. Zeidat, and Z. Zhao, "Supervised clustering-algorithms and benefits," in Proceedings of the IEEE International Conference on Tools with Artificial Intelligence, 2004, pp. 774-776.

[201] C. F. Eick, B. Vaezian, D. Jiang, and J. Wang, "Discovery of interesting regions in spatial data sets using supervised clustering," in Proceedings of the European Conference on Principles of Data Mining and Knowledge Discovery, 2006, pp. 127-138.

[202] S. H. Al-Harbi and V. J. Rayward-Smith, "Adapting k-means for supervised clustering," Applied Intelligence, vol. 24, no. 3, pp. 219-226, 2006. 
[203] O. A. Ismaili, V. Lemaire, and A. Cornuejols, "Supervised pre-processings are useful for supervised clustering," in Analysis of Large and Complex Data, 2016, pp. 147-157.

[204] P. K. Amalaman, C. F. Eick, and C. Wang, "Supervised taxonomies, algorithms and applications," IEEE Transactions on Knowledge and Data Engineering, vol. 29, no. 9, pp. 2040-2052, 2017.

[205] J. Wu, C. Leng, Y. Wang, Q. Hu, and J. Cheng, "Quantized convolutional neural networks for mobile devices," in Proceedings of the IEEE Conference on Computer Vision and Pattern Recognition, 2016, pp. 4820-4828.

[206] M. Denil, B. Shakibi, L. Dinh, M. Ranzato, and N. De Freitas, "Predicting parameters in deep learning," in Proceedings of the Advances in Neural Information Processing Systems, 2013, pp. 2148-2156.

[207] J. Luo, H. Zhang, H. Zhou, C. Xie, J. Wu, and W. Lin, "ThiNet: Pruning CNN filters for a thinner net," IEEE Transactions on Pattern Analysis and Machine Intelligence, vol. 41, no. 10, pp. 2525-2538, 2019.

[208] M. C. Mozer and P. Smolensky, "Skeletonization: A technique for trimming the fat from a network via relevance assessment," in Proceedings of the Advances in Neural Information Processing Systems, vol. 1, 1988, pp. 107-115.

[209] R. Reed, "Pruning algorithms: A survey," IEEE Transactions on Neural Networks, vol. 4, no. 5, pp. 740-747, 1993.

[210] Y. LeCun, J. S. Denker, and S. A. Solla, "Optimal brain damage," in Proceedings of the Advances in Neural Information Processing Systems, 1990, pp. 598-605.

[211] B. Hassibi and D. G. Stork, "Second order derivatives for network pruning: Optimal brain surgeon," in Proceedings of the Advances in Neural Information Processing Systems, 1993, pp. 164-171.

[212] A. S. Weigend, D. E. Rumelhart, and B. A. Huberman, "Generalization by weightelimination applied to currency exchange rate prediction," in Proceedings of the IEEE International Joint Conference on Neural Networks, 1991, pp. 2374-2379. 
[213] S. Hanson and L. Pratt, "Comparing biases for minimal network construction with backpropagation," in Proceedings of the Advances in Neural Information Processing Systems, 1988, pp. 177-185.

[214] A. S. Weigend, D. E. Rumelhart, and B. A. Huberman, "Back-propagation, weightelimination and time series prediction," in Connectionist models, 1991, pp. 105-116.

[215] S. Han, J. Pool, J. Tran, and W. Dally, "Learning both weights and connections for efficient neural network," in Proceedings of the Advances in Neural Information Processing Systems, 2015, pp. 1135-1143.

[216] Z. Liu, M. Sun, T. Zhou, G. Huang, and T. Darrell, "Rethinking the value of network pruning," in Proceedings of the International Conference on Learning Representations, 2019.

[217] D. C. Mocanu, E. Mocanu, P. Stone, P. H. Nguyen, M. Gibescu, and A. Liotta, "Scalable training of artificial neural networks with adaptive sparse connectivity inspired by network science," Nature communications, vol. 9, no. 1, p. 2383, 2018.

[218] J. Frankle, G. K. Dziugaite, D. M. Roy, and M. Carbin, "Stabilizing the lottery ticket hypothesis," CoRR, vol. abs/1903.01611, 2019.

[219] A. Morcos, H. Yu, M. Paganini, and Y. Tian, "One ticket to win them all: generalizing lottery ticket initializations across datasets and optimizers," in Proceedings of the Advances in Neural Information Processing Systems, 2019, pp. 4932-4942.

[220] N. Hubens, M. Mancas, M. Decombas, M. Preda, T. Zaharia, B. Gosselin, and T. Dutoit, "An experimental study of the impact of pre-training on the pruning of a convolutional neural network," in Proceedings of the International Conference on Applications of Intelligent Systems, 2020, pp. 1-6.

[221] H. Zhou, J. Lan, R. Liu, and J. Yosinski, "Deconstructing lottery tickets: Zeros, signs, and the supermask," in Proceedings of the Advances in Neural Information Processing Systems, 2019, pp. 3597-3607.

[222] H. Yu, S. Edunov, Y. Tian, and A. S. Morcos, "Playing the lottery with rewards and multiple languages: lottery tickets in $\mathrm{rl}$ and nlp," in International Conference on Learning Representations, 2020. 
[223] V. Lebedev and V. Lempitsky, "Fast ConvNets using group-wise brain damage," in Proceedings of the IEEE Conference on Computer Vision and Pattern Recognition, 2016, pp. 2554-2564.

[224] H. Zhou, J. M. Alvarez, and F. Porikli, "Less is more: Towards compact CNNs," in Proceedings of the European Conference on Computer Vision, 2016, pp. 662-677.

[225] T. He, Y. Fan, Y. Qian, T. Tan, and K. Yu, "Reshaping deep neural network for fast decoding by node-pruning," in Proceedings of the International Conference on Acoustics, Speech and Signal Processing, 2014, pp. 245-249.

[226] S. Srinivas and R. V. Babu, "Data-free parameter pruning for deep neural networks," in Proceedings of the British Machine Vision Conference, 2015, pp. 31.1-31.12.

[227] Z. Mariet and S. Sra, "Diversity networks: Neural network compression using determinantal point processes," in Proceedings of the International Conference on Learning Representations, 2016.

[228] H. Li, A. Kadav, I. Durdanovic, H. Samet, and H. P. Graf, "Pruning filters for efficient ConvNets," in Proceedings of the International Conference on Learning Representations, 2017.

[229] C. Liu and H. Wu, "Channel pruning based on mean gradient for accelerating convolutional neural networks," Signal Processing, vol. 156, pp. 84-91, 2019.

[230] A. Polyak and L. Wolf, "Channel-level acceleration of deep face representations," IEEE Access, no. 3, pp. 2163-2175, 2015.

[231] J.-H. Luo and J. Wu, "An entropy-based pruning method for cnn compression," CoRR, vol. abs/1706.05791, 2017.

[232] H. Hu, R. Peng, Y.-W. Tai, and C.-K. Tang, "Network trimming: A data-driven neuron pruning approach towards efficient deep architectures," CoRR, vol. abs/1607.03250, 2016.

[233] S.-K. Yeom, P. Seegerer, S. Lapuschkin, S. Wiedemann, K.-R. Müller, and W. Samek, "Pruning by explaining: A novel criterion for deep neural network pruning," Pattern Recognition, p. 107899, 2021. 
[234] X. Ding, G. Ding, Y. Guo, and J. Han, "Centripetal SGD for pruning very deep convolutional networks with complicated structure," in Proceedings of the IEEE Conference on Computer Vision and Pattern Recognition, 2019, pp. 4943-4953.

[235] Y. He, G. Kang, X. Dong, Y. Fu, and Y. Yang, "Soft filter pruning for accelerating deep convolutional neural networks," in Proceedings of the International Joint Conference on Artificial Intelligence, 2018, pp. 2234-2240.

[236] Y. He, P. Liu, Z. Wang, Z. Hu, and Y. Yang, "Filter pruning via geometric median for deep convolutional neural networks acceleration," in Proceedings of the IEEE Conference on Computer Vision and Pattern Recognition, 2019, pp. 4340-4349.

[237] Z. Liu, H. Mu, X. Zhang, Z. Guo, X. Yang, K.-T. Cheng, and J. Sun, "Metapruning: Meta learning for automatic neural network channel pruning," in Proceedings of the IEEE International Conference on Computer Vision, 2019, pp. 3296-3305.

[238] Z. You, K. Yan, J. Ye, M. Ma, and P. Wang, "Gate decorator: Global filter pruning method for accelerating deep convolutional neural networks," in Proceedings of the Advances in Neural Information Processing Systems, 2019, pp. 2133-2144.

[239] J.-H. Luo and J. Wu, "Autopruner: An end-to-end trainable filter pruning method for efficient deep model inference," Pattern Recognition, p. 107461, 2020.

[240] M. Rastegari, V. Ordonez, J. Redmon, and A. Farhadi, "XNOR-Net: Imagenet classification using binary convolutional neural networks," in Proceedings of the European conference on computer vision, 2016, pp. 525-542.

[241] Y. Zhao, X. Gao, D. Bates, R. Mullins, and C.-Z. Xu, "Focused quantization for sparse CNNs," in Proceedings of the Advances in Neural Information Processing Systems, 2019, pp. 5584-5593.

[242] A. Zhou, A. Yao, Y. Guo, L. Xu, and Y. Chen, "Incremental network quantization: Towards lossless CNNs with low-precision weights," CoRR, vol. abs/1702.03044, 2017.

[243] Y. Gong, L. Liu, M. Yang, and L. Bourdev, "Compressing deep convolutional networks using vector quantization," CoRR, vol. abs/1412.6115, 2014. 
[244] V. Vanhoucke, A. Senior, and M. Z. Mao, "Improving the speed of neural networks on cpus," in Proceedings of the NIPS Workshop on Deep Learning and Unsupervised Feature Learning, 2011.

[245] S. Gupta, A. Agrawal, K. Gopalakrishnan, and P. Narayanan, "Deep learning with limited numerical precision," in Proceedings of the International Conference on Machine Learning, 2015, pp. 1737-1746.

[246] S. Han, H. Mao, and W. J. Dally, "Deep compression: Compressing deep neural networks with pruning, trained quantization and Huffman coding," in Proceedings of the International Conference on Learning Representations, 2016.

[247] K. Ullrich, E. Meeds, and M. Welling, "Soft weight-sharing for neural network compression," in Proceedings of the International Conference on Learning Representations, 2017.

[248] W. Chen, J. Wilson, S. Tyree, K. Weinberger, and Y. Chen, "Compressing neural networks with the hashing trick," in Proceedings of the International Conference on Machine Learning, 2015, pp. 2285-2294.

[249] M. Courbariaux, I. Hubara, D. Soudry, R. El-Yaniv, and Y. Bengio, "Binarized neural networks: Training deep neural networks with weights and activations constrained to +1 or -1," CoRR, vol. abs/1602.02830, 2016.

[250] M. Courbariaux, Y. Bengio, and J.-P. David, "Binaryconnect: Training deep neural networks with binary weights during propagations," in Proceedings of the Advances in Neural Information Processing Systems, 2015, pp. 3123-3131.

[251] L. Hou, Q. Yao, and J. T. Kwok, "Loss-aware binarization of deep networks," in Proceedings of the International Conference on Learning Representations, 2017.

[252] Z. Lin, M. Courbariaux, R. Memisevic, and Y. Bengio, "Neural networks with few multiplications," in Proceedings of the International Conference on Learning Representations, 2016.

[253] A. Sironi, B. Tekin, R. Rigamonti, V. Lepetit, and P. Fua, "Learning separable filters," in Proceedings of the IEEE Conference on Computer Vision and Pattern Recognition, 2013, pp. 2754-2761. 
[254] E. L. Denton, W. Zaremba, J. Bruna, Y. LeCun, and R. Fergus, "Exploiting linear structure within convolutional networks for efficient evaluation," in Proceedings of the Advances in Neural Information Processing Systems, 2014, pp. 1269-1277.

[255] M. Jaderberg, A. Vedaldi, and A. Zisserman, "Speeding up convolutional neural networks with low rank expansions," in Proceedings of the British Machine Vision Conference, 2014.

[256] T. Sainath, B. Kingsbury, V. Sindhwani, E. Arisoy, and B. Ramabhadran, "Low-rank matrix factorization for deep neural network training with high-dimensional output targets," in Proceedings of the IEEE International Conference on Acoustics, Speech and Signal Processing, 2013, pp. 6655-6659.

[257] Y. Lu, A. Kumar, S. Zhai, Y. Cheng, T. Javidi, and R. Feris, "Fully-adaptive feature sharing in multi-task networks with applications in person attribute classification," in Proceedings of the IEEE Conference on Computer Vision and Pattern Recognition, 2017, pp. 1131-1140.

[258] V. Lebedev, Y. Ganin, M. Rakhuba, I. Oseledets, and V. Lempitsky, "Speeding-up convolutional neural networks using fine-tuned CP-Decomposition," in Proceedings of the International Conference on Learning Representations, 2015.

[259] C. Tai, T. Xiao, X. Wang, and E. Weinan, "Convolutional neural networks with low-rank regularization," in Proceedings of the International Conference on Learning Representations, 2016.

[260] J. J. Hull, "A database for handwritten text recognition research," IEEE Transactions on Pattern Analysis and Machine Intelligence, vol. 16, no. 5, pp. 550-554, 1994.

[261] H. Xiao, K. Rasul, and R. Vollgraf, "Fashion-MNIST: a novel image dataset for benchmarking machine learning algorithms," CoRR, vol. abs/1708.07747, 2017.

[262] M. Wu and B. Schölkopf, "A local learning approach for clustering," in Proceedings of the Advances in Neural Information Processing Systems, 2007, pp. 1529-1536.

[263] W. Y. Chen, Y. Song, H. Bai, C. J. Lin, and E. Y. Chang, "Parallel spectral clustering in distributed systems," IEEE Transactions on Pattern Analysis and Machine Intelligence, vol. 33 , no. 3 , pp. 568-586, 2010 
[264] A. Strehl and J. Ghosh, "Cluster ensembles-a knowledge reuse framework for combining multiple partitions," Journal of Machine Learning Research, vol. 3, pp. 583-617, 2002.

[265] F. Chollet et al., "Keras," 2015. [Online]. Available: https://github.com/fchollet/keras

[266] X. Guo, E. Zhu, X. Liu, and J. Yin, "Deep embedded clustering with data augmentation," in Proceedings of the Asian Conference on Machine Learning, 2018, pp. 550-565.

[267] L. v. d. Maaten and G. Hinton, "Visualizing data using t-SNE," Journal of Machine Learning Research, vol. 9, pp. 2579-2605, 2008.

[268] O. Kilinc and I. Uysal, "GAR: an efficient and scalable graph-based activity regularization for semi-supervised learning," Neurocomputing, vol. 296, pp. 46-54, 2018.

[269] Theano Development Team, "Theano: A Python framework for fast computation of mathematical expressions," CoRR, vol. abs/1605.02688, 2016.

[270] Y. Netzer, T. Wang, A. Coates, A. Bissacco, B. Wu, and A. Y. Ng, "Reading digits in natural images with unsupervised feature learning," in Proceedings of the NIPS Workshop on Deep Learning and Unsupervised Feature Learning, 2011.

[271] O. Kilinc and I. Uysal, "Learning latent representations in neural networks for clustering through pseudo supervision and graph-based activity regularization," in Proceedings of the International Conference on Learning Representations, 2018.

[272] — - "Auto-clustering output layer: Automatic learning of latent annotations in neural networks," CoRR, vol. abs/1702.08648, 2017.

[273] A. Dosovitskiy, P. Fischer, J. T. Springenberg, M. Riedmiller, and T. Brox, "Discriminative unsupervised feature learning with exemplar convolutional neural networks," IEEE Transactions on Pattern Analysis and Machine Intelligence, vol. 38, no. 9, pp. 17341747, 2016.

[274] K. Kavukcuoglu, R. Fergus, Y. LeCun et al., "Learning invariant features through topographic filter maps," in Proceedings of the IEEE Conference on Computer Vision and Pattern Recognition, 2009, pp. 1605-1612. 
[275] M. Raghu, B. Poole, J. Kleinberg, S. Ganguli, and J. S. Dickstein, "On the expressive power of deep neural networks," in Proceedings of the International Conference on Machine Learning, 2017, pp. 2847-2854.

[276] G. Urban, K. J. Geras, S. E. Kahou, O. Aslan, S. Wang, R. Caruana, A. Mohamed, M. Philipose, and M. Richardson, "Do deep convolutional nets really need to be deep and convolutional?" in Proceedings of the International Conference on Learning Representations, 2017.

[277] A. Krizhevsky and G. Hinton, "Learning multiple layers of features from tiny images," Technical report, University of Toronto, 2009. [Online]. Available: https://www.cs.utoronto.ca/ kriz/learning-features-2009-TR.pdf

[278] C. Wah, S. Branson, P. Welinder, P. Perona, and S. Belongie, "The Caltech-UCSD Birds200-2011 Dataset," Technical report, California Institute of Technology, 2011. [Online]. Available: http://www.vision.caltech.edu/visipedia/papers/CUB_200_2011.pdf

[279] O. Russakovsky, J. Deng, H. Su, J. Krause, S. Satheesh, S. Ma, Z. Huang, A. Karpathy, A. Khosla, M. Bernstein et al., "Imagenet large scale visual recognition challenge," International Journal of Computer Vision, vol. 115, pp. 211-252, 2015.

[280] M. Guillaumin, D. Küttel, and V. Ferrari, "Imagenet auto-annotation with segmentation propagation," International Journal of Computer Vision, vol. 110, pp. 328-348, 2014.

[281] S. Liu and W. Deng, "Very deep convolutional neural network based image classification using small training sample size," in Proceedings of the IAPR Asian Conference on Pattern Recognition, 2015, pp. 730-734.

[282] S. Lin, R. Ji, Y. Li, C. Deng, and X. Li, "Toward compact convnets via structure-sparsity regularized filter pruning," IEEE Transactions on Neural Networks and Learning Systems, vol. 31, no. 2, pp. 574-588, 2019.

[283] Z. Zhuang, M. Tan, B. Zhuang, J. Liu, Y. Guo, Q. Wu, J. Huang, and J. Zhu, "Discrimination-aware channel pruning for deep neural networks," in Proceedings of the Advances in Neural Information Processing Systems, 2018, pp. 875-886.

[284] W. Aigner, S. Miksch, H. Schumann, and C. Tominski, Visualization of time-oriented data. Springer Publishing Company, Incorporated, 2011. 
[285] I. Hotz and R. Peikert, "Definition of a multifield," in Scientific Visualization, 2014, pp. $105-109$.

[286] E. Lughofer and M. Sayed-Mouchaweh, Predictive Maintenance in Dynamic Systems: Advanced Methods, Decision Support Tools and Real-World Applications. Springer, 2019.

[287] E. Lughofer, A.-C. Zavoianu, R. Pollak, M. Pratama, P. Meyer-Heye, H. Zörrer, C. Eitzinger, and T. Radauer, "Autonomous supervision and optimization of product quality in a multi-stage manufacturing process based on self-adaptive prediction models," Journal of Process Control, vol. 76, pp. 27-45, 2019.

[288] F. Battke, S. Symons, and K. Nieselt, "Mayday-integrative analytics for expression data," BMC Bioinformatics, vol. 11, no. 1, p. 121, 2010.

[289] D. H. Jeong, A. Darvish, K. Najarian, J. Yang, and W. Ribarsky, "Interactive visual analysis of time-series microarray data," The Visual Computer, vol. 24, no. 12, pp. 10531066, 2008.

[290] A. Vogogias, J. Kennedy, and D. Archambault, "Hierarchical clustering with multipleheight branch-cut applied to short time-series gene expression data," in Proceedings of the EuroVis, 2016.

[291] M. Cho, B. Kim, H.-J. Bae, and J. Seo, "Stroscope: Multi-scale visualization of irregularly measured time-series data," IEEE Transactions on Visualization and Computer Graphics, vol. 20, no. 5, pp. 808-821, 2014.

[292] R. Chang, M. Ghoniem, R. Kosara, W. Ribarsky, J. Yang, E. Suma, C. Ziemkiewicz, D. Kern, and A. Sudjianto, "Wirevis: Visualization of categorical, time-varying data from financial transactions," in Proceedings of the IEEE Symposium on Visual Analytics Science and Technology, 2007, pp. 155-162.

[293] C. Xie, W. Chen, X. Huang, Y. Hu, S. Barlowe, and J. Yang, "VAET: A visual analytics approach for e-transactions time-series," IEEE Transactions on Visualization and Computer Graphics, vol. 20, no. 12, pp. 1743-1752, 2014.

[294] C. Turkay, E. Kaya, S. Balcisoy, and H. Hauser, "Designing progressive and interactive analytics processes for high-dimensional data analysis," IEEE Transactions on Visualization and Computer Graphics, vol. 23, no. 1, pp. 131-140, 2017. 
[295] S. T. Lei and K. Zhang, "A visual analytics system for financial time-series data," in Proceedings of the International Symposium on Visual Information Communication, 2010, pp. 1-9.

[296] T. Schreck, J. Bernard, T. Von Landesberger, and J. Kohlhammer, "Visual cluster analysis of trajectory data with interactive kohonen maps," in Information Visualization, 2009, pp. 14-29.

[297] T. Schreck, T. Tekušová, J. Kohlhammer, and D. Fellner, "Trajectory-based visual analysis of large financial time series data," ACM SIGKDD Explorations Newsletter, vol. 9, no. 2, pp. 30-37, 2007.

[298] A. Perer and J. Sun, "Matrixflow: temporal network visual analytics to track symptom evolution during disease progression," in Proceedings of the AMIA Annual Symposium, vol. 2012, 2012, pp. 716-725.

[299] S. Guo, K. Xu, R. Zhao, D. Gotz, H. Zha, and N. Cao, "Eventthread: Visual summarization and stage analysis of event sequence data," IEEE Transactions on Visualization and Computer Graphics, vol. 24, no. 1, pp. 56-65, 2018.

[300] Y. Wang, T. Wu, Z. Chen, Q. Luo, and H. Qu, "Stac: Enhancing stacked graphs for time series analysis," in Proceedings of the IEEE Pacific Visualization Symposium, 2016, pp. 234-238.

[301] L. Wilkinson, "Visualizing big data outliers through distributed aggregation," IEEE Transactions on Visualization and Computer Graphics, no. 1, pp. 1-1, 2018.

[302] A. Meidiana and S.-H. Hong, "Multistory: Visual analytics of dynamic multi-relational networks," in Proceedings of the IEEE Pacific Visualization Symposium, 2015, pp. 7579.

[303] S. Hadlak, H. Schumann, C. H. Cap, and T. Wollenberg, "Supporting the visual analysis of dynamic networks by clustering associated temporal attributes," IEEE Transactions on Visualization and Computer Graphics, vol. 19, no. 12, pp. 2267-2276, 2013.

[304] M. Steiger, J. Bernard, S. Mittelstadt, H. Lucke-Tieke, D. Keim, T. May, and J. Kohlhammer, "Visual analysis of time-series similarities for anomaly detection in sensor networks," Computer Graphics Forum, vol. 33, no. 3, pp. 401-410, 2014. 
[305] S. van den Elzen, D. Holten, J. Blaas, and J. J. van Wijk, "Reducing snapshots to points: A visual analytics approach to dynamic network exploration," IEEE Transactions on Visualization and Computer Graphics, vol. 22, no. 1, pp. 1-10, 2016.

[306] F. Zhou, W. Huang, Y. Zhao, Y. Shi, X. Liang, and X. Fan, "Entvis: A visual analytic tool for entropy-based network traffic anomaly detection," IEEE Computer Graphics and Applications, vol. 35, no. 6, pp. 42-50, 2015.

[307] T. Fujiwara, J. K. Li, M. Mubarak, C. Ross, C. D. Carothers, R. B. Ross, and K.-L. Ma, "A visual analytics system for optimizing the performance of large-scale networks in supercomputing systems," Visual Informatics, vol. 2, no. 1, pp. 98-110, 2018.

[308] N. Cao, C. Shi, S. Lin, J. Lu, Y.-R. Lin, and C.-Y. Lin, "Targetvue: Visual analysis of anomalous user behaviors in online communication systems," IEEE Transactions on Visualization and Computer Graphics, vol. 22, no. 1, pp. 280-289, 2016.

[309] M. C. Hao, M. Marwah, H. Janetzko, D.-i. A. Keim, U. Dayal, R. Sharma, D. Patnaik, and N. Ramakrish-nan, "Visual analysis of frequent patterns in large time series," in Proceedings of the IEEE Symposium on Visual Analytics Science and Technology, 2010, pp. 227-228.

[310] C. Muelder, B. Zhu, W. Chen, H. Zhang, and K.-L. Ma, "Visual analysis of cloud computing performance using behavioral lines," IEEE Transactions on Visualization and Computer Graphics, vol. 22, no. 6, pp. 1694-1704, 2016.

[311] G. Sharma, G. Shroff, A. Pandey, B. Singh, G. Sehgal, K. Paneri, and P. Agarwal, "Multi-sensor visual analytics supported by machine-learning models," in Proceedings of the International Conference on Data Mining Workshop, 2015, pp. 668-674.

[312] L. Shi, Q. Liao, Y. He, R. Li, A. Striegel, and Z. Su, "Save: Sensor anomaly visualization engine," in Proceedings of the IEEE Conference on Visual Analytics Science and Technology, 2011, pp. 201-210.

[313] C. Arbesser, F. Spechtenhauser, T. Mühlbacher, and H. Piringer, "Visplause: Visual data quality assessment of many time series using plausibility checks," IEEE Transactions on Visualization and Computer Graphics, vol. 23, no. 1, pp. 641-650, 2017. 
[314] Y. Chen, P. Xu, and L. Ren, "Sequence synopsis: Optimize visual summary of temporal event data," IEEE Transactions on Visualization and Computer Graphics, vol. 24, no. 1, pp. 45-55, 2018.

[315] G. Andrienko, N. Andrienko, and S. Wrobel, "Visual analytics tools for analysis of movement data," ACM SIGKDD Explorations Newsletter, vol. 9, no. 2, pp. 38-46, 2007.

[316] G. Andrienko and N. Andrienko, "Spatio-temporal aggregation for visual analysis of movements," in Proceedings of the IEEE Symposium on Visual Analytics Science and Technology, 2008, pp. 51-58.

[317] G. Andrienko, N. Andrienko, C. Hurter, S. Rinzivillo, and S. Wrobel, "From movement tracks through events to places: Extracting and characterizing significant places from mobility data," in Proceedings of the IEEE Conference on Visual Analytics Science and Technology, 2011, pp. 161-170.

[318] G. Andrienko, N. Andrienko, C. Hurter, S. Rinzivillo, and S. Wrobel, "Scalable analysis of movement data for extracting and exploring significant places," IEEE Transactions on Visualization and Computer Graphics, vol. 19, no. 7, pp. 1078-1094, 2013.

[319] M. Lu, Z. Wang, and X. Yuan, "Trajrank: Exploring travel behaviour on a route by trajectory ranking," in Proceedings of the IEEE Pacific Visualization Symposium, 2015, pp. 311-318.

[320] G. Andrienko, N. Andrienko, S. Rinzivillo, M. Nanni, D. Pedreschi, and F. Giannotti, "Interactive visual clustering of large collections of trajectories," in Proceedings of the IEEE Symposium on Visual Analytics Science and Technology, 2009, pp. 3-10.

[321] M. Riveiro, M. Lebram, and M. Elmer, "Anomaly detection for road traffic: A visual analytics framework," IEEE Transactions on Intelligent Transportation Systems, vol. 18, no. 8, pp. 2260-2270, 2017.

[322] I. Kalamaras, A. Zamichos, A. Salamanis, A. Drosou, D. D. Kehagias, G. Margaritis, S. Papadopoulos, and D. Tzovaras, "An interactive visual analytics platform for smart intelligent transportation systems management," IEEE Transactions on Intelligent Transportation Systems, vol. 19, no. 2, pp. 487-496, 2018. 
[323] G. Andrienko, N. Andrienko, M. Mladenov, M. Mock, and C. Pölitz, "Identifying place histories from activity traces with an eye to parameter impact," IEEE Transactions on Visualization and Computer Graphics, vol. 18, no. 5, pp. 675-688, 2012.

[324] G. L. Andrienko, N. V. Andrienko, G. Fuchs, A.-M. O. Raimond, J. Symanzik, and C. Ziemlicki, "Extracting semantics of individual places from movement data by analyzing temporal patterns of visits." in Proceedings of the ACM SIGSPATIAL International Workshop on Computational Models of Place, 2013, pp. 9-15.

[325] J. Chae, G. Wang, B. Ahlbrand, M. B. Gorantla, J. Zhang, S. Chen, H. Xu, J. Zhao, W. Hatton, A. Malik et al., "Visual analytics of heterogeneous data for criminal event analysis vast challenge 2015: Grand challenge," in Proceedings of the IEEE Conference on Visual Analytics Science and Technology, 2015, pp. 149-150.

[326] J. Pu, S. Liu, P. Xu, H. Qu, and L. M. Ni, "Mviewer: mobile phone spatiotemporal data viewer," Frontiers of Computer Science, vol. 8, no. 2, pp. 298-315, 2014.

[327] Z. Shen and K.-L. Ma, "Mobivis: A visualization system for exploring mobile data," in Proceedings of the IEEE Pacific Visualization Symposium, 2008, pp. 175-182.

[328] J. Zhao, G. Wang, J. Chae, H. Xu, S. Chen, W. Hatton, S. Towers, M. B. Gorantla, B. Ahlbrand, J. Zhang et al., "Parkanalyzer: Characterizing the movement patterns of visitors vast 2015 mini-challenge 1," in Proceedings of the IEEE Conference on Visual Analytics Science and Technology, 2015, pp. 179-180.

[329] T. von Landesberger, F. Brodkorb, P. Roskosch, N. Andrienko, G. Andrienko, and A. Kerren, "Mobilitygraphs: Visual analysis of mass mobility dynamics via spatiotemporal graphs and clustering," IEEE Transactions on Visualization and Computer Graphics, vol. 22, no. 1, pp. 11-20, 2016.

[330] A. Biswas, G. Lin, X. Liu, and H.-W. Shen, "Visualization of time-varying weather ensembles across multiple resolutions," IEEE Transactions on Visualization and Computer Graphics, vol. 23, no. 1, pp. 841-850, 2017.

[331] H. Senaratne, M. Mueller, M. Behrisch, F. Lalanne, J. Bustos-Jiménez, J. Schneidewind, D. Keim, and T. Schreck, "Urban mobility analysis with mobile network data: A visual analytics approach," IEEE Transactions on Intelligent Transportation Systems, vol. 19, no. 5, pp. 1537-1546, 2018. 
[332] L. Stopar, P. Skraba, M. Grobelnik, and D. Mladenic, "Streamstory: Exploring multivariate time series on multiple scales," IEEE Transactions on Visualization and Computer Graphics, 2018.

[333] L. Gao, H. A. Campbell, O. R. Bidder, and J. Hunter, "A web-based semantic tagging and activity recognition system for species' accelerometry data," Ecological Informatics, vol. 13, pp. 47-56, 2013.

[334] J. S. Walker, M. W. Jones, R. S. Laramee, O. R. Bidder, H. J. Williams, R. Scott, E. L. Shepard, and R. P. Wilson, "Timeclassifier: a visual analytic system for the classification of multi-dimensional time series data," The Visual Computer, vol. 31, no. 6-8, pp. 10671078, 2015.

[335] J. Bernard, N. Wilhelm, M. Scherer, T. May, and T. Schreck, "Timeseriespaths: projection-based explorative analysis of multivariate time series data," Journal of WSCG, pp. 97-106, 2012.

[336] R. Kincaid and H. Lam, "Line graph explorer: scalable display of line graphs using focus+ context," in Proceedings of the Conference on Advanced Visual Interfaces, 2006, pp. 404-411.

[337] J. Li, Z. Xiao, H.-Q. Zhao, Z.-P. Meng, and K. Zhang, "Visual analytics of smogs in china," Journal of Visualization, vol. 19, no. 3, pp. 461-474, 2016.

[338] J. Li, K. Zhang, and Z.-P. Meng, "Vismate: Interactive visual analysis of station-based observation data on climate changes," in Proceedings of Conference Visual Analytics Science and Technology, 2014, pp. 133-142.

[339] S. Martin and T.-T. Quach, "Interactive visualization of multivariate time series data," in Proceedings of the International Conference on Augmented Cognition, 2016, pp. 322332.

[340] Q. Shu, H. Guo, J. Liang, L. Che, J. Liu, and X. Yuan, "Ensemblegraph: Interactive visual analysis of spatiotemporal behaviors in ensemble simulation data," in Proceedings of the IEEE Pacific Visualization Symposium, 2016, pp. 56-63.

[341] W. Wu, Y. Zheng, H. Qu, W. Chen, E. Gröller, and L. M. Ni, "Boundaryseer: Visual analysis of $2 \mathrm{~d}$ boundary changes," in Proceedings of the IEEE Conference on Visual Analytics Science and Technology, 2014, pp. 143-152. 
[342] T. Blascheck, M. John, K. Kurzhals, S. Koch, and T. Ertl, "Va 2: A visual analytics approach for evaluating visual analytics applications," IEEE Transactions on Visualization and Computer Graphics, vol. 22, no. 1, pp. 61-70, 2016.

[343] O. Purwantiningsih, A. Sallaberry, S. Andary, A. Seilles, and J. Aze, "Visual analysis of body movement in serious games for healthcare," in Proceedings of the IEEE Pacific Visualization Symposium, 2016, pp. 229-233.

[344] J. G. Kim, M. Snodgrass, M. Pietrowicz, and K. Karahalios, "Visual analysis of relationships between behavioral and physiological sensor data," in Proceedings of the International Conference on Healthcare Informatics, 2015, pp. 170-179.

[345] C. Turkay, J. Parulek, N. Reuter, and H. Hauser, "Interactive visual analysis of temporal cluster structures," Computer Graphics Forum, vol. 30, no. 3, pp. 711-720, 2011.

[346] A. Soriano-Vargas, B. C. Vani, M. H. Shimabukuro, J. F. Monico, M. C. F. Oliveira, and B. Hamann, "Visual analytics of time-varying multivariate ionospheric scintillation data," Computers \& Graphics, vol. 68, pp. 96-107, 2017.

[347] G. Trajcevski, D. Gunopulos, C. C. Aggarwal, and C. Reddy, "Time-series data clustering," in Data Clustering: Algorithms and Applications, 2013, pp. 357-375.

[348] Z. Xing, J. Pei, and E. Keogh, "A brief survey on sequence classification," $A C M$ SIGKDD Explorations Newsletter, vol. 12, no. 1, pp. 40-48, 2010.

[349] B. D. Fulcher and N. S. Jones, "Highly comparative feature-based time-series classification," IEEE Transactions on Knowledge and Data Engineering, vol. 26, no. 12, pp. 3026-3037, 2014.

[350] M. Tucci and M. Raugi, "Analysis of spectral clustering algorithms for linear and nonlinear time series," in Proceedings of the International Conference on Intelligent Systems Design and Applications, 2011, pp. 925-930.

[351] F. Petitjean, A. Ketterlin, and P. Gançarski, "A global averaging method for dynamic time warping, with applications to clustering," Pattern Recognition, vol. 44, no. 3, pp. 678-693, 2011. 
[352] J. Yang and J. Leskovec, "Patterns of temporal variation in online media," in Proceedings of the ACM International Conference on Web Search and Data Mining, 2011, pp. 177-186.

[353] J. Paparrizos and L. Gravano, "k-shape: Efficient and accurate clustering of time series," in Proceedings of the ACM SIGMOD International Conference on Management of Data, 2015, pp. 1855-1870.

[354] L. N. Ferreira and L. Zhao, "Time series clustering via community detection in networks," Information Sciences, vol. 326, pp. 227-242, 2016.

[355] C. Guo, H. Jia, and N. Zhang, "Time series clustering based on ica for stock data analysis," in Proceedings of the International Conference on Wireless Communications, Networking and Mobile Computing, 2008, pp. 1-4.

[356] J. Zakaria, A. Mueen, and E. Keogh, "Clustering time series using unsupervisedshapelets," in Proceedings of the International Conference on Data Mining, 2012, pp. 785-794.

[357] L. Shi, L. Du, and Y.-D. Shen, "Robust spectral learning for unsupervised feature selection," in Proceedings of the International Conference on Data Mining, 2014, pp. 977-982.

[358] M. Qian and C. Zhai, "Robust unsupervised feature selection," in Proceedings of the International Joint Conference on Artificial Intelligence, 2013, pp. 1621-1627.

[359] Z. Li, Y. Yang, J. Liu, X. Zhou, and H. Lu, "Unsupervised feature selection using nonnegative spectral analysis," in Proceedings of the AAAI Conference on Artificial Intelligence, 2012, pp. 1026-1032.

[360] Y. Yang, H. T. Shen, Z. Ma, Z. Huang, and X. Zhou, " 2, 1-norm regularized discriminative feature selection for unsupervised learning," in Proceedings of the International Joint Conference on Artificial Intelligence, 2011, pp. 1589 - 1594.

[361] Q. Ma, S. Li, L. Shen, J. Wang, J. Wei, Z. Yu, and G. Cottrell, "End-to-end incomplete time-series modeling from linear memory of latent variables," IEEE Transactions on Cybernetics, vol. 50, pp. 4908-4920, 2020. 
[362] H. Lei, Y. Xia, and X. Qin, "Estimation of semivarying coefficient time series models with arma errors," Annals of Statistics, vol. 44, pp. 1618-1660, 2016.

[363] Z. Cai, J. Fan, and Q. Yao, "Functional-coefficient regression models for nonlinear time series," Journal of the American Statistical Association, vol. 95, pp. 941 - 956, 1999.

[364] D. Tjostheim and B. Auestad, "Nonparametric identification of nonlinear time series: Projections," Journal of the American Statistical Association, vol. 89, pp. 1398-1409, 1994.

[365] C. O. S. Sorzano, J. Vargas, and A. P. Montano, "A survey of dimensionality reduction techniques," CoRR, vol. abs/1403.2877, 2014.

[366] X. Wang, A. Mueen, H. Ding, G. Trajcevski, P. Scheuermann, and E. Keogh, "Experimental comparison of representation methods and distance measures for time series data," Data Mining and Knowledge Discovery, vol. 26, no. 2, pp. 275-309, 2013.

[367] K. Tornai, L. Kovács, A. Oláh, R. Drenyovszki, I. Pintér, D. Tisza, and J. Levendovszky, "Classification for consumption data in smart grid based on forecasting time series," Electric Power Systems Research, vol. 141, pp. 191-201, 2016.

[368] H. Yahyaoui and A. Al-Mutairi, "A feature-based trust sequence classification algorithm," Information Sciences, vol. 328, pp. 455-484, 2016.

[369] L. Wei and E. Keogh, "Semi-supervised time series classification," in Proceedings of the International Conference on Knowledge Discovery and Data Mining, 2006, pp. 748753.

[370] J. Ye, C. Xiao, R. M. Esteves, and C. Rong, "Time series similarity evaluation based on spearman's correlation coefficients and distance measures," in Proceedings of the International Conference on Cloud Computing and Big Data in Asia, 2015, pp. 319331.

[371] P. Buono, A. Aris, C. Plaisant, A. Khella, and B. Shneiderman, "Interactive pattern search in time series," in Electronic Imaging, 2005, pp. 175-186.

[372] D. Yu, X. Yu, Q. Hu, J. Liu, and A. Wu, "Dynamic time warping constraint learning for large margin nearest neighbor classification," Information Sciences, vol. 181, no. 13, pp. 2787-2796, 2011. 
[373] J. Zhao and L. Itti, "shapedtw: shape dynamic time warping," Pattern Recognition, vol. 74, no. C, pp. 171-184, 2018.

[374] C. A. Ratanamahatana and E. Keogh, "Making time-series classification more accurate using learned constraints," in Proceedings of the SIAM International Conference on Data Mining, 2004, pp. 11-22.

[375] A. Mueen and E. Keogh, "Extracting optimal performance from dynamic time warping," in Proceedings of the International Conference on Knowledge Discovery and Data Mining, 2016, pp. 2129-2130.

[376] M. Kotas, J. M. Leski, and T. Moron, "Dynamic time warping based on modified alignment costs for evoked potentials averaging," in Man-Machine Interactions 4, 2016, pp. 305-314.

[377] C. Faloutsos, M. Ranganathan, and Y. Manolopoulos, Fast subsequence matching in time-series databases. ACM, 1994, vol. 23, no. 2.

[378] D. J. Berndt and J. Clifford, "Using dynamic time warping to find patterns in time series." in Proceedings of the Workshop on Knowledge Discovery in Databases, 1994, pp. 359-370.

[379] E. Keogh and C. A. Ratanamahatana, "Exact indexing of dynamic time warping," Knowledge and Information Systems, vol. 7, no. 3, pp. 358-386, 2005.

[380] M. Vlachos, G. Kollios, and D. Gunopulos, "Discovering similar multidimensional trajectories," in Proceedings of the International Conference on Data Engineering, 2002, pp. 673-684.

[381] S. Smith, Digital signal processing: a practical guide for engineers and scientists. Elsevier, 2013.

[382] H. Kaya and Ş. Gündüz-Öğüdücü, "A distance based time series classification framework," Information Systems, vol. 51, pp. 27-42, 2015.

[383] K. Yang and C. Shahabi, "A pca-based similarity measure for multivariate time series," in Proceedings of the ACM International Workshop on Multimedia Databases, 2004, pp. $65-74$. 
[384] A. Singhal and D. E. Seborg, "Clustering multivariate time-series data," Journal of Chemometrics, vol. 19, no. 8, pp. 427-438, 2005.

[385] K. Yang and C. Shahabi, "On the stationarity of multivariate time series for correlationbased data analysis," in Proceedings of the International Conference on Data Mining, 2005, pp. 4-pp.

[386] R. H. Lesch, Y. Caillé, and D. Lowe, "Component analysis in financial time series," in Proceedings of the Conference on Computational Intelligence for Financial Engineering, 1999, pp. 183-190.

[387] T. Lin, F. Guo, Y. Wu, B. Zhu, F. Zhang, H. Qu, and W. Chen, "Tievis: Visual analytics of evolution of interpersonal ties," in Proceedings of the International Conference on Technologies for E-Learning and Digital Entertainment, 2016, pp. 412-424.

[388] G. Dong and J. Pei, Sequence Data Mining. Springer, 2007, vol. 33.

[389] T.-c. Fu, "A review on time series data mining," Engineering Applications of Artificial Intelligence, vol. 24, no. 1, pp. 164-181, 2011.

[390] R. Agrawal, C. Faloutsos, and A. Swami, "Efficient similarity search in sequence databases," in Proceedings of the International Conference on Foundations of Data Organization and Algorithms, 1993, pp. 69-84.

[391] D. Rafiei and A. O. Mendelzon, "Querying time series data based on similarity," IEEE Transactions on Knowledge and Data Engineering, vol. 12, no. 5, pp. 675-693, 2000.

[392] G. J. Janacek, A. J. Bagnall, and M. Powell, "A likelihood ratio distance measure for the similarity between the fourier transform of time series," in Proceedings of the Pacific Asia Conference on Knowledge Discovery and Data Mining, 2005, pp. 737-743.

[393] I. Popivanov and R. J. Miller, "Similarity search over time-series data using wavelets," in Proceedings of the International Conference on Data Engineering, 2002, pp. 212-221.

[394] K.-P. Chan and A. W.-C. Fu, "Efficient time series matching by wavelets," in Proceedings of the International Conference on Data Engineering, 1999, pp. 126-133.

[395] C. C. Aggarwal, "On effective classification of strings with wavelets," in Proceedings of the International Conference on Knowledge Discovery and Data Mining, 2002, pp. $163-172$. 
[396] D. Li, T. F. D. A. Bissyande, J. Klein, and Y. Le Traon, "Time series classification with discrete wavelet transformed data: Insights from an empirical study," in Proceedings of the International Conference on Software Engineering and Knowledge Engineering, 2016, pp. 1361-1377.

[397] L. Ye and E. Keogh, "Time series shapelets: a new primitive for data mining," in Proceedings of the International Conference on Knowledge discovery and Data Mining, 2009, pp. 947-956.

[398] Z. Xing, J. Pei, S. Y. Philip, and K. Wang, "Extracting interpretable features for early classification on time series." in Proceedings of the SIAM International Conference on Data Mining, vol. 11, 2011, pp. 247-258.

[399] V. Niennattrakul and C. A. Ratanamahatana, "On clustering multimedia time series data using k-means and dynamic time warping," in Proceedings of the International Conference on Multimedia and Ubiquitous Engineering, 2007, pp. 733-738.

[400] W. Meesrikamolkul, V. Niennattrakul, and C. A. Ratanamahatana, "Shape-based clustering for time series data," in Proceedings of the Pacific Asia Conference on Knowledge Discovery and Data Mining, 2012, pp. 530-541.

[401] V. Hautamaki, P. Nykanen, and P. Franti, "Time-series clustering by approximate prototypes," in Proceedings of the International Conference on Pattern Recognition, 2008, pp. $1-4$.

[402] K. Kalpakis, D. Gada, and V. Puttagunta, "Distance measures for effective clustering of arima time-series," in Proceedings of the International Conference on Data Mining, 2001, pp. 273-280.

[403] X. Golay, S. Kollias, G. Stoll, D.-e. Meier, A. Valavanis, and P. Boesiger, "A new correlation-based fuzzy logic clustering algorithm for fmri," Magnetic Resonance in Medicine, vol. 40, no. 2, pp. 249-260, 1998.

[404] P. D'Urso, C. Cappelli, D. D. Lallo, and R. Massari, "Clustering of financial time series," Physica A: Statistical Mechanics and its Applications, vol. 392, no. 9, pp. 2114-2129, 2013. 
[405] J. J. Van Wijk and E. R. Van Selow, "Cluster and calendar based visualization of time series data," in Proceedings of the IEEE Symposium on Information Visualization, 1999, pp. 4-9.

[406] M. Simonsen, T. Mailund, and C. N. S. Pedersen, "Rapid neighbour-joining," in Proceedings of the International Workshop on Algorithms in Bioinformatics, 2008, pp. 113 122.

[407] D. Sacha, M. Kraus, J. Bernard, M. Behrisch, T. Schreck, Y. Asano, and D. A. Keim, "Somflow: Guided exploratory cluster analysis with self-organizing maps and analytic provenance," IEEE Transactions on Visualization and Computer Graphics, vol. 24, no. 1, pp. 120-130, 2018.

[408] M. Varstal, J. D. R. Millán, and J. Heikkonen, "A recurrent self-organizing map for temporal sequence processing," in Proceedings of the International Conference on Artificial Neural Networks, 1997, pp. 421-426.

[409] T. Voegtlin, "Recursive self-organizing maps," Neural Networks, vol. 15, no. 8, pp. 979991, 2002.

[410] T.-c. Fu, F.-1. Chung, V. Ng, and R. Luk, "Pattern discovery from stock time series using self-organizing maps," in Proceedings of the Workshop on Temporal Data Mining, 2001, pp. 26-29.

[411] Z. Cui, W. Chen, and Y. Chen, "Multi-scale convolutional neural networks for time series classification,” CoRR, vol. abs/1603.06995, 2016.

[412] C. Antunes and A. Oliveira, "Temporal data mining: An overview," in Proceedings of the KDD Workshop on Temporal Data Mining, 2001, pp. 26-29.

[413] Y. Zheng, Q. Liu, E. Chen, Y. Ge, and J. L. Zhao, "Exploiting multi-channels deep convolutional neural networks for multivariate time series classification," Frontiers of Computer Science, vol. 10, no. 1, pp. 96-112, 2016.

[414] J. S. Walker, M. W. Jones, R. S. Laramee, M. D. Holton, E. L. Shepard, H. J. Williams, D. M. Scantlebury, J. M. Nikki, E. A. Magowan, I. E. Maguire et al., "Prying into the intimate secrets of animal lives; software beyond hardware for comprehensive annotation in 'daily diary'tags," Movement Ecology, vol. 3, no. 1, p. 29, 2015. 
[415] O. Bidder, J. Walker, M. Jones, M. Holton, P. Urge, D. Scantlebury, N. Marks, E. Magowan, I. Maguire, and R. Wilson, "Step by step: reconstruction of terrestrial animal movement paths by dead-reckoning," Movement Ecology, vol. 3, no. 1, p. 23, 2015.

[416] R. P. Wilson, E. Shepard, and N. Liebsch, "Prying into the intimate details of animal lives: use of a daily diary on animals," Endangered Species Research, vol. 4, no. 1-2, pp. 123-137, 2008.

[417] W. F. Fagan, M. A. Lewis, M. Auger-Méthé, T. Avgar, S. Benhamou, G. Breed, L. LaDage, U. E. Schlägel, W.-w. Tang, Y. P. Papastamatiou et al., "Spatial memory and animal movement," Ecology letters, vol. 16, no. 10, pp. 1316-1329, 2013.

[418] O. R. Bidder, L. A. Qasem, and R. P. Wilson, "On higher ground: how well can dynamic body acceleration determine speed in variable terrain?" PloS one, vol. 7, no. 11, 2012.

[419] A. Vedaldi and K. Lenc, "Matconvnet: Convolutional neural networks for matlab," in Proceedings of the ACM Conference on Multimedia, 2015, pp. 689-692.

[420] T. Thinsungnoen, K. Kerdprasop, and N. Kerdprasop, "Deep autoencoder networks optimized with genetic algorithms for efficient ecg clustering," International Journal of Machine Learning and Computing, vol. 8, no. 2, pp. 112-116, 2018.

[421] M. Sun, Y. Wang, F. Teng, Y. Ye, G. Strbac, and C. Kang, "Clustering-based residential baseline estimation: A probabilistic perspective," IEEE Transactions on Smart Grid, vol. 10, pp. 6014-6028, 2019.

[422] J. de Jong, M. A. Emon, P. Wu, R. Karki, M. Sood, P. Godard, A. Ahmad, H. A. Vrooman, M. Hofmann-Apitius, and H. Frohlich, "Deep learning for clustering of multivariate clinical patient trajectories with missing values," GigaScience, vol. 8, 2019.

[423] R. Asadi and A. Regan, "Spatio-temporal clustering of traffic data with deep embedded clustering," in Proceedings of the ACM SIGSPATIAL International Workshop on Prediction of Human Mobility, 2019, pp. 45-52.

[424] N. S. Madiraju, S. Sadat, D. Fisher, and H. Karimabadi, "Deep temporal clustering : Fully unsupervised learning of time-domain features," CoRR, vol. abs/1802.01059, 2018. 
[425] M. P. Wachowiak, J. J. Moggridge, and R. Wachowiak-Smolikova, "Deep embedded clustering for data-driven ecg exploration using continuous wavelet transforms," in Proceedings of the International Conference on Information and Digital Technologies, 2019, pp. 551-556.

[426] P. Wolf, A. Chin, and B. Baker, "Unsupervised data-driven automotive diagnostics with improved deep temporal clustering," in Proceedings of the Vehicular Technology Conference, 2019, pp. 1-6.

[427] G. Zhang, A. R. Singer, and N. Vlahopoulos, "Temporal clustering network for selfdiagnosing faults from vibration measurements," CoRR, vol. abs/2006.09505, 2020.

[428] G. Richard, B. Grossin, G. Germaine, G. Hebrail, and A. de Moliner, "Autoencoderbased time series clustering with energy applications," CoRR, vol. abs/2002.03624, 2020 .

[429] S. M. Mousavi, W. Zhu, W. Ellsworth, and G. Beroza, "Unsupervised clustering of seismic signals using deep convolutional autoencoders," IEEE Geoscience and Remote Sensing Letters, vol. 16, no. 11, pp. 1693-1697, 2019.

[430] S. Ryu, H. Choi, H. Lee, H. Kim, and V. S. Wong, "Residential load profile clustering via deep convolutional autoencoder," in Proceedings of the IEEE International Conference on Communications, Control, and Computing Technologies for Smart Grids, 2018, pp. $1-6$.

[431] C. Liu, C. Liu, F. Liu, and J. Hu, "Clustering analysis of urban fabric detection based on mobile traffic data," in Journal of Physics: Conference Series, vol. 1453, 2020, p. 012158 .

[432] D. Ienco and R. Interdonato, "Deep multivariate time series embedding clustering via attentive-gated autoencoder," in Proceedings of the Pacific-Asia Conference on Knowledge Discovery and Data Mining, 2020, pp. 318-329.

[433] M. Yue, Y. Li, H. Yang, R. Ahuja, Y.-Y. Chiang, and C. Shahabi, "Detect: Deep trajectory clustering for mobility-behavior analysis," in Proceedings of the International Conference on Big Data, 2019, pp. 988-997. 
[434] C. Lee and M. V. D. Schaar, "Temporal phenotyping using deep predictive clustering of disease progression," in Proceedings of the International Conference on Machine learning, 2020, pp. 5767-5777.

[435] K. Luxem, F. Fuhrmann, J. Kursch, S. Remy, and P. Bauer, "Identifying behavioral structure from deep variational embeddings of animal motion," Preprint at bioRxiv, 2020. [Online]. Available: https://doi.org/10.1101/2020.05.14.095430

[436] A. Abedin, F. Motlagh, Q. Shi, H. Rezatofighi, and D. Ranasinghe, "Towards deep clustering of human activities from wearables," in Proceedings of the International Symposium on Wearable Computers, 2020, p. 1-6.

[437] Q. Ma, J. Zheng, S. Li, and G. Cottrell, "Learning representations for time series clustering," in Proceedings of the Advances in Neural Information Processing Systems, 2019.

[438] N. Khan, M. Ahmed, and N. Roy, "Temporal clustering based thermal condition monitoring in building," Sustainable Computing: Informatics and Systems, p. 100441, 2020.

[439] L. Han, K. Zheng, L. Zhao, X. Wang, and X. Shen, "Short-term traffic prediction based on deepcluster in large-scale road networks," IEEE Transactions on Vehicular Technology, vol. 68, pp. 12 301-12313, 2019.

[440] K. Greff, R. K. Srivastava, J. Koutnik, B. R. Steunebrink, and J. Schmidhuber, "Lstm: A search space odyssey," IEEE Transactions on Neural Networks and Learning Systems, vol. 28, no. 10, pp. 2222-2232, 2016.

[441] K. Cho, B. Van Merrienboer, C. Gulcehre, D. Bahdanau, F. Bougares, H. Schwenk, and Y. Bengio, "Learning phrase representations using rnn encoder-decoder for statistical machine translation," CoRR, vol. abs/1406.1078, 2014.

[442] I. Sutskever, O. Vinyals, and Q. V. Le, "Sequence to sequence learning with neural networks," in Proceedings of the Advances in Neural Information Processing Systems, 2014, pp. 3104-3112.

[443] L. Logeswaran and H. Lee, "An efficient framework for learning sentence representations," in Proceedings of the International Conference on Learning Representations, 2018. 
[444] R. Kiros, Y. Zhu, R. R. Salakhutdinov, R. Zemel, R. Urtasun, A. Torralba, and S. Fidler, "Skip-thought vectors," in Proceedings of the Advances in Neural Information Processing Systems, 2015, pp. 3294-3302.

[445] Z. Gan, Y. Pu, R. Henao, C. Li, X. He, and L. Carin, "Learning generic sentence representations using convolutional neural networks," in Proceedings of the Conference on Empirical Methods in Natural Language Processing, 2017, pp. 2390-2400.

[446] S. Mukherjee, H. Asnani, E. Lin, and S. Kannan, "Clustergan: Latent space clustering in generative adversarial networks," in Proceedings of the AAAI Conference on Artificial Intelligence, vol. 33, 2019, pp. 4610-4617.

[447] K. E. Smith and A. O. Smith, "Conditional gan for timeseries generation," CoRR, vol. abs/2006.16477, 2020.

[448] M. Cuturi and M. Blondel, "Soft-dtw: a differentiable loss function for time-series," in Proceedings of the International Conference on Machine Learning, 2017, pp. 894-903.

[449] H. Sakoe and S. Chiba, "Dynamic programming algorithm optimization for spoken word recognition," IEEE Transactions on Acoustics, Speech, and Signal Processing, pp. 4349, 1978

[450] R. A. S. Weber, M. Eyal, N. Skafte, O. Shriki, and O. Freifeld, "Diffeomorphic temporal alignment nets," in Proceedings of the Advances in Neural Information Processing Systems, 2019, pp. 6574-6585.

[451] M. Edwards, J. Deng, and X. Xie, "From pose to activity: Surveying datasets and introducing converse," Computer Vision and Image Understanding, vol. 144, pp. 73-105, 2016.

[452] H. A. Dau, A. Bagnall, K. Kamgar, C.-C. M. Yeh, Y. Zhu, S. Gharghabi, C. A. Ratanamahatana, and E. Keogh, "The ucr time series archive," Journal of Automatica Sinica, vol. 6, no. 6, pp. 1293-1305, 2019.

[453] A. Javed, B. S. Lee, and D. M. Rizzo, "A benchmark study on time series clustering," Machine Learning with Applications, p. 100001, 2020.

[454] D. Zeng, F. Zhao, W. Shen, and S. Ge, "Compressing and accelerating neural network for facial point localization," Cognitive Computation, pp. 359-367, 2018. 
[455] S. Ge, "Efficient deep learning in network compression and acceleration," in Digital Systems. IntechOpen, 2018. 